\title{
accelerator and fusion research division
}

\section{Summary of Activities}

Lawrence Berkeley Laboratory

University of California

1 Cyclotron Road

Berkeley, CA 94720

December 1992

This work was supported principally by the U.S. Department of Energy under Contract No. DE-AC03-76SF00098. Support came from the Director, Office of Energy Research, through the following offices:

- Office of High Energy and Nuclear Physics (High Energy Technology Division, Nuclear Physics Division, and High Energy Physics Division).

- Office of Fusion Energy (Applied Plasma Physics Division and Development and Technology Division).

- Office of Basic Energy Sciences (Advanced Energy Projects Division, Engineering and Geosciences Division, and Materials Sciences Division).

- Office of Energy Research Analysis (Assessment Projects Division).

- Office of Superconducting Super Collider.

Additional support came from the U.S. Department of Defense through the Strategic Defense Initiative Organization (U.S. Army Strategic Defense Command), the Air Force Office of Scientific Research, the Air Force Space Technology Center, the Air Force Weapons Laboratory, the U.S. Army Research Office, and the Office of Naval Research. 


\section{Contents}

Foreword

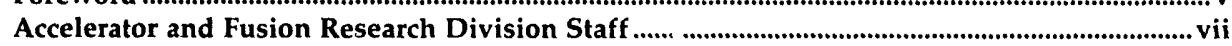

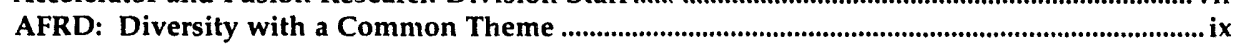

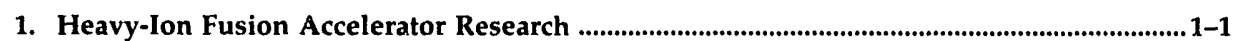

Induction Linac Systems Experiments .... .................................................................... 1-3

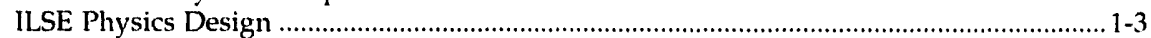

Ion-Source and Injector Development ................................................................... 1-7

ILSE Transport Physics Studies on SBTE ............................................................... 1-8

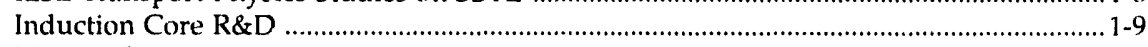

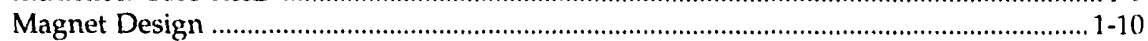

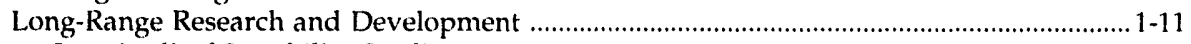

Longitudinal Instability Studies ........................................................................ 1-11

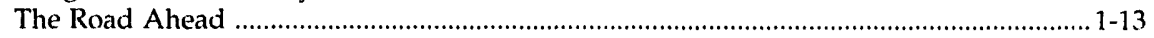

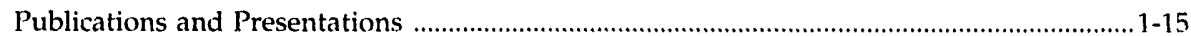

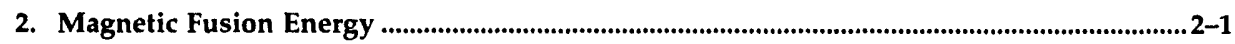

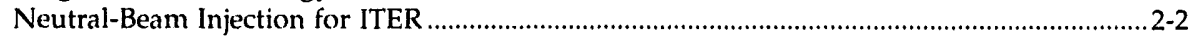

Accelerator Proof-Of-Principle Experiment .............................................................2-2

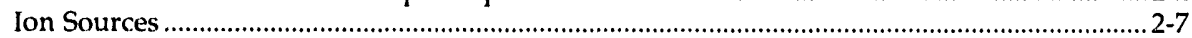

Progress with Surface-Conversion Sources ...............................................................2-7

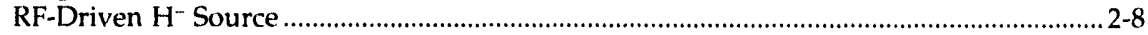

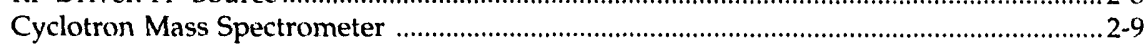

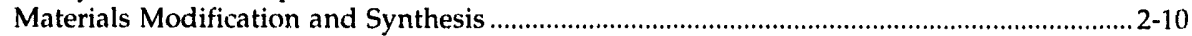

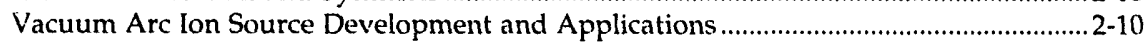

Metallic Thin Films and Multilayers ............................................................................2-11

Metal Plasma Immersion (MPl) Surface Modification ................................................. 2-11

Diamond Synthesis ........................................................................................... 2-11

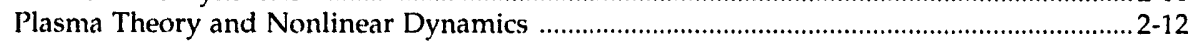

Wave Dynamics and Gyroresonant Energy Absorption ..........................................2-12

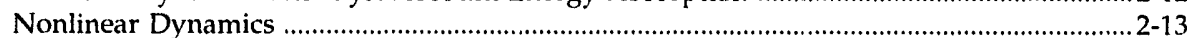

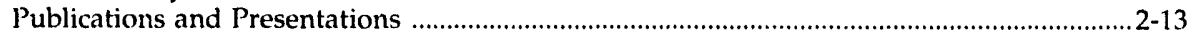

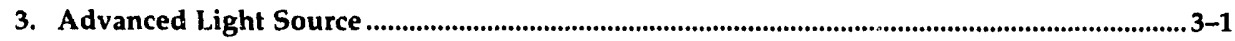

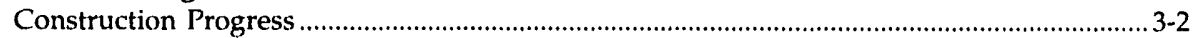

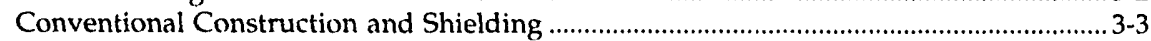

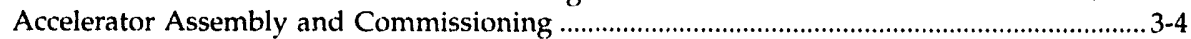

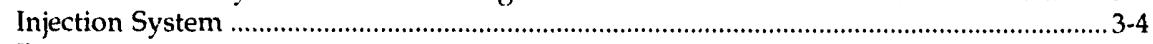

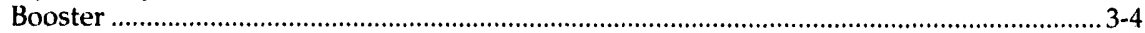

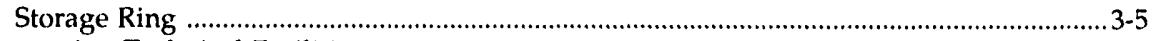

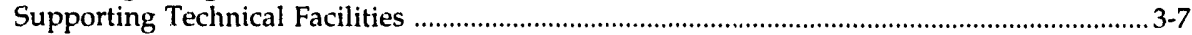

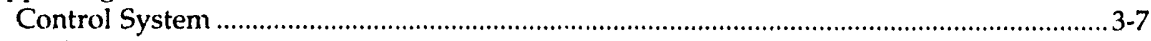

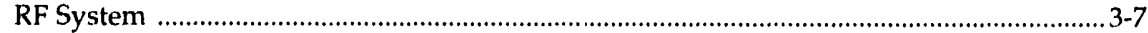

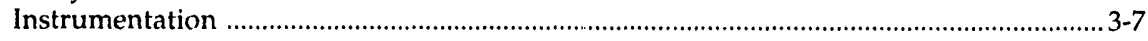

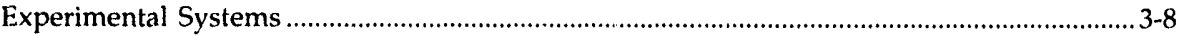

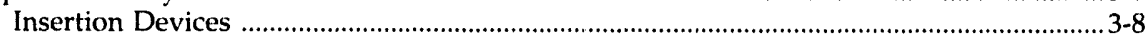

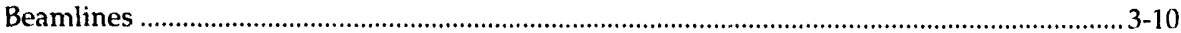

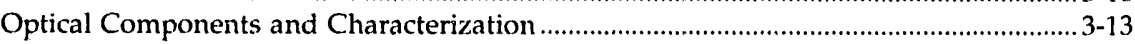

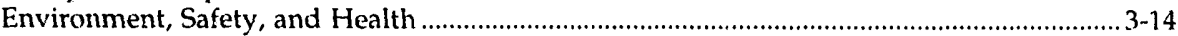

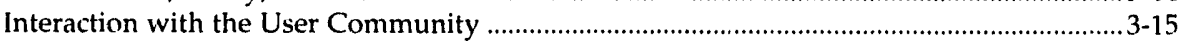

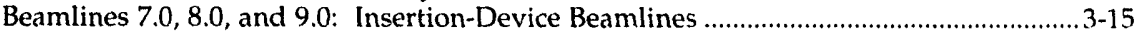

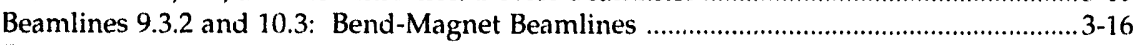

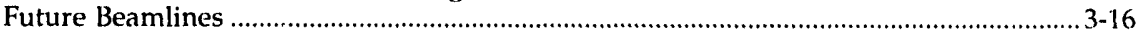

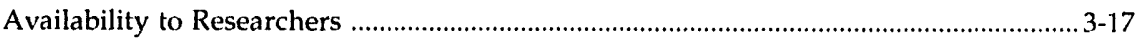

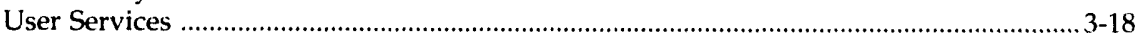

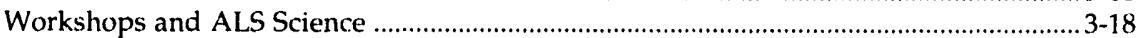

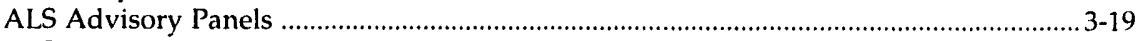

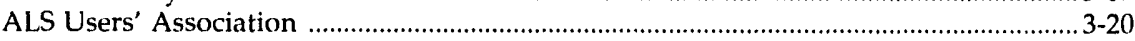

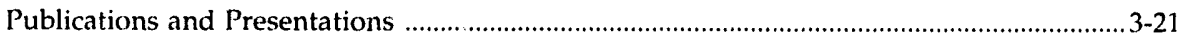




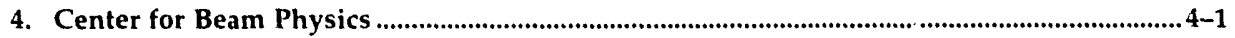

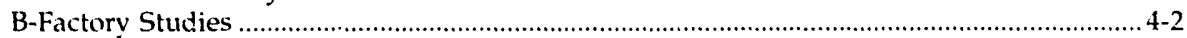

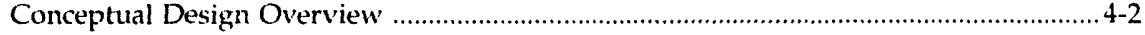

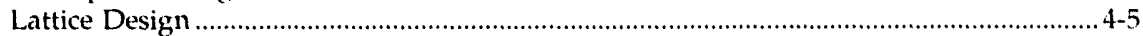

Beam-Beam Interaction Studies ................................................................................. 4-5

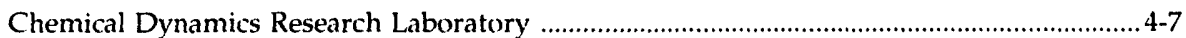

CDRL: A Unique Combination of User Facilities ..........................................................4-8

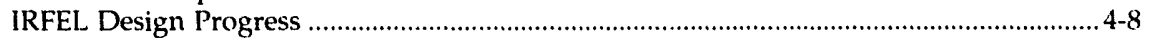

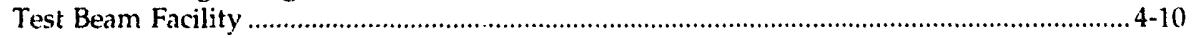

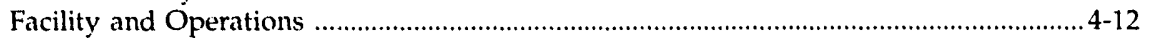

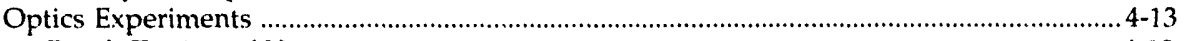

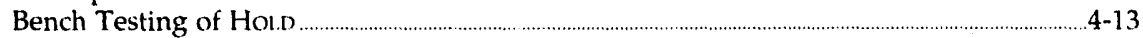

Advanced Optical Diagnostics for FELs ............................................................................4-14

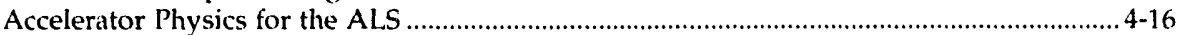

RF Measurements and Feedback Systems ....................................................................... 4-16

Injector Commissioning Experience ........................................................................... 4-17

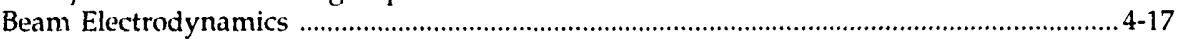

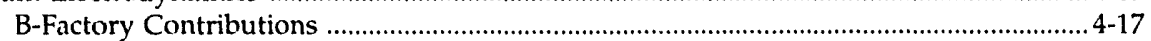

Fermilab Antiproton Cooling System ........................................................................... 4-19

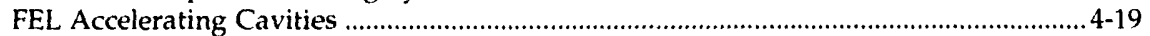

High-Energy Collider Physics .................................................................................................. 4-19

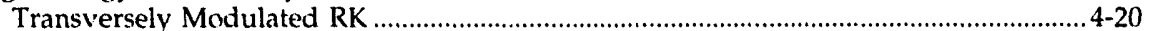

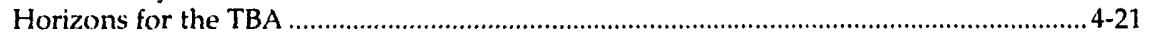

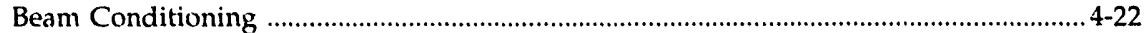

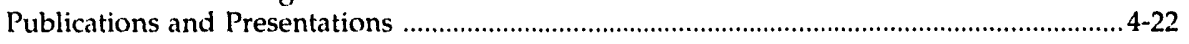

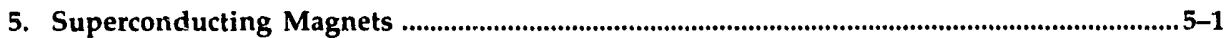

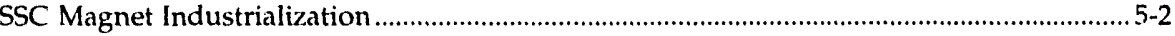

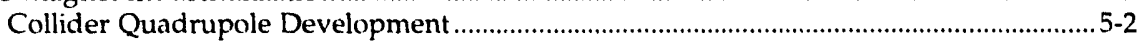

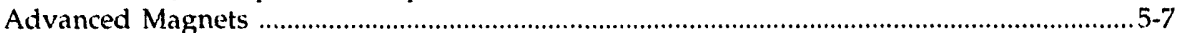

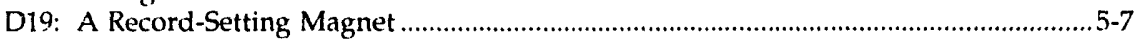

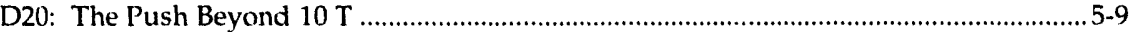

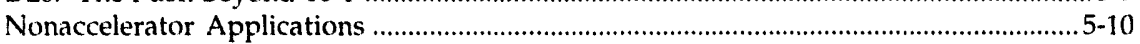

Advanced Materials and Design Techniques ..................................................................5-10

APC Materials Development ...................................................................................... 5-10

High-T Superconductor at Low Temperature ........................................................... 5-13

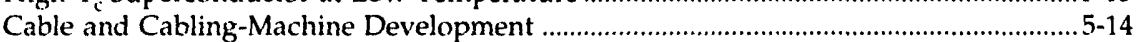

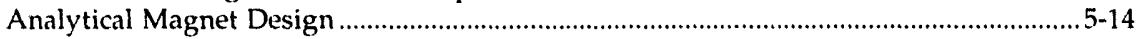

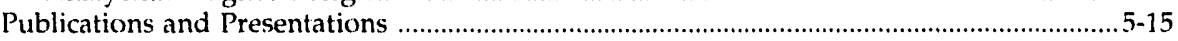

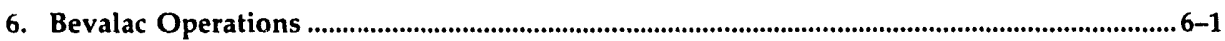

Accelerator Technology and Operations Summary ..................................................................6-3

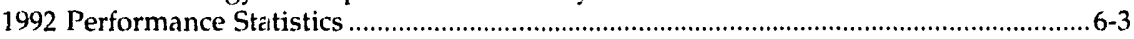

Facility Development Projects ...............................................................................................6-4

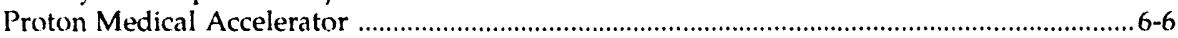

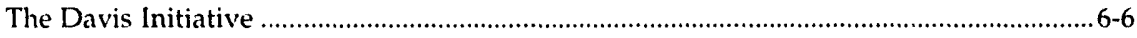

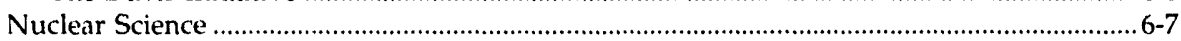

EOS Studies and the Time Projection Chamber ...................................................................6-7

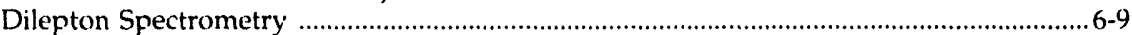

Neutrons and Light Charged Particles ..................................................................6-12

Grazing Collisions and Secondary Radioactive Beams ..............................................6-12

Nuclear Astrophysics, Atomic Physics, and NASA Instrument Calibration ...................6-13

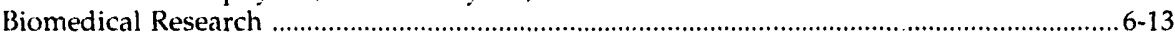

Heavy-Ion Radiotherapy and Radiosurgery .............................................................. 6-14

Radiation Biology and Biophysics Research .............................................................6-16

Equipment Development and Related Biomedical Research ............................................6-19

Publications and Presentations ................................................................................6-21 


\section{Foreword}

Nineteen ninety-two was a busy, profuctive year of research and planning in anticipation of future projects. The Induction Linac Systems Experiments, the logical next step on the road to a heavy-ion "driver" for inertial fusion energy, received a Determination of Need from the Department of Energya key vote of confidence and an optimistic note on which to submit a proposal this year for a 1995 construction start. Meanwhile, our research in magnetic fusion energy progressed further with a design for an accelerator proof-of-principle experiment. The resulting accelerator, at larger scale, would be at the heart of the neutral-beam plasma heaters might be used in the International Thermonuclear Experimental Reactor (ITER). Both ITER and heavy-ion drivers are important elements in the DOE's National Energy Strategy, and we are pleased to be able to contribute to their advancement.

The Advanced Light Source (ALS), a national user facility designed to be at the forefront of synchrotron-radiation research well into the 21st century, is being completed and comnissioned by stages in preparation for the beginning of operations in spring 1993. The first beam will soon be injected into the storage ring; meanwhile, the "Beamline Scorecard" shows that, of the 11 beamlines planned for the period through 1995, three insertion-device and two bend-magnet beamlines are expected to be in use for data-taking during the first year of ALS operations. The availability of high-brightness ultraviolet and $x$-ray beams from the ALS is anticipated eagerly in a broad variety of research communities, including materials science, chemical dynamics, and structural biology. To expand the use of the ALS, a Beamlines Initiative was proposed to the DOE. The initiative includes four more of the insertion devices that enhance synchrotron-light production, along with their associated beamlines, plus completion of second-floor laboratory and office space.

Approval of the proposed Beam Physics Facility was among the highlights of our Exploratory Studies Program. The facility, whose design has been finalized, will use the high-quality $50-\mathrm{MeV}$ electron beam from the ALS injection linac for a diverse experimental program during the periods when the storage ring is full and the injector would otherwise be idle. Of the many possible experiments, the first will be a plasma focus and a scheme for generating femtosecond $X$-ray pulses with Compton scattering against a laser beam. We also further refined two proposed initiatives: PEP-II, a "B factory" at the Stanford Linear Accelerator Center, and an infrared freeelectron laser for the Chemical Dynamics Research Laboratory.

Our program in superconducting magnets concluded the SSC Magnet Industrialization Program, in which representatives of private industry worked alongside LBL personnel to learn how to build the LBL-designed collider quadrupoles for the Superconducting Super Collider. Research and development aimed at materials and magnets for the future resulted in a world record for field strength in an accelerator-type magnet (a central field of just over 10 teslas), and among our new lines of inquiry is an effort to develop much-stronger magnets. 
Not all the news was good, as we learned that DOE support for the Bevalac heavy-ion accelerator complex would end midway through fiscal year 1993. Plans are being made and implemented for the optimum redeployment of Bevalac personnel (the ALS is one of the facilities already taking advantage of their expertise). We are also looking at ways to make the best use of this heavy laboratory space and to perform the decommissioning in a safe, environmentally responsible, and cost-effective manner. Yet even as the end draws near for this venerable and enormously productive accelerator, the spinoffs from its development and operation continue; most prominently, we have been involved in the design of a proton-therapy initiative put forth by the University of California-Davis Medical Center.

AFRD researchers continued receiving awards and peer recognition in 1992, including the R\&D 100 awards, Research and Development Magcizine's recognition for the year's 100 most significant technical innovations. Three longtime collaborators in the Bevalac biomedical program-AFRD's Tim Renner, along with Bill Chu and Bernhard Ludewigt of the Life Sciences Division-won with the Raster Scanner Beam Delivery System. Ian Brown of AFRD's magnetic fusion energy program, together with Robert MacGill, Michael Dickinson, and James Galvin of the Engineering Division, earned another R\&D 100 for the DC Broad-Beam, High Current Metal Ion Source. Meanwhile, a Federal Laboratory Consortium Technology Transfer Award went to Wayne McKinney of the ALS Experimental Systems Group in recognition of his efforts, in collaboration with industry, to develop and characterize supersmooth metal surfaces for synchrotron-radiation optics.

Behind all these plans and achievements are the people of AFRD. Their 1992 accomplishments, including the highlights described in this report, were made possible by a unique combination of skill, creativity, and hard work. These qualities have made me proud to serve as their leader, and I am confident that the permanent director, when appointed, will inherit a forefront scientific organization worthy of the LBL tradition.

Richard A. Gough

Acting Director,

Accelerator and Fusion Research Division

December 1992 


\section{Accelerator and Fusion Research Division Staff}

Richard A. Gough, Acting Division Director

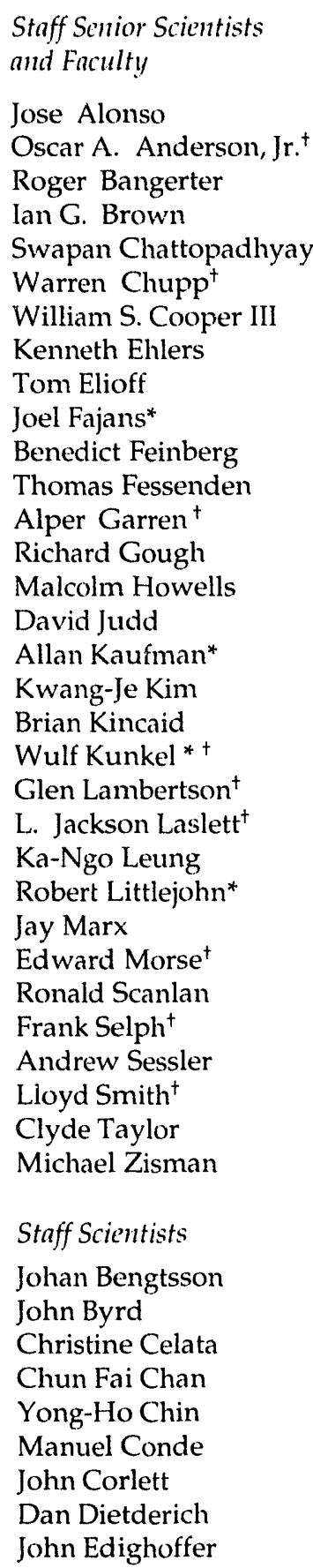

William Everette

Shmuel Eylon

William Fawley

Robert Force

Etienne Forest

Miguel Furman

Basil Gavin ${ }^{\dagger}$

David Goldberg

Kyoung Hahn

William Hassenzahl

Philip Heimann

Alan Jackson

Juris Kalnins

Roderich Keller

Ardith Kenney

Charles Kim

Gary Krebs

Srinivas Krishnagopal

Joe Kwan

Edward Lee

Wim Leemans

Alan Lietzke

Fred Lothrop

Wayne McKinney

Malika Meddahi

Margaret McMah..n

Miklos Melczer

Hircshi Nishimura

Rupert Perera

Govindan Rangarajan

Joseph Rechen

Timothy Renner

Robert Rimmer

David Robin

Arthur Robinson

Henry Rutkowski

Lindsay Schachinger

Alfred Schlachter

Peter Seidl

John Staples

William Steele

Simon $Y u$

Johannes Van Oort

Michael Vella

Anthony Warwick

Ming Xie

\author{
Anthony Young \\ Alexander Zholents \\ Postdoctoral Fellow \\ Stephen Creagh \\ Graduate Student Resenrch \\ Assistants \\ Hector Beguiristain \\ Daniel Cook \\ William Flynn \\ Richa Govil \\ Charles Hansen \\ Christopher Jarzynski \\ Kenneth Lamon \\ James Morehead \\ Khanh Nguyen \\ Tai Nguyen \\ Max Wei \\ Operators and Technical \\ Support Persominel
}

Glenn Ackerman

Robert Aita

Thomas Althar

Ronald Barr

Douglas Bentsen

James Brannigan

Robert Brokloff

Warren Byrne

Royce Callaway ${ }^{\dagger}$

Robert Coates ${ }^{+}$

Donald Cowles ${ }^{\dagger}$

Suzanne Daly ${ }^{\dagger}$

Hugh Ellison ${ }^{\dagger}$

Thomas Gimpel ${ }^{\dagger}$

Cheryl Hauck

Paul Howell ${ }^{\dagger}$

Orland Jones

Thomas Kutz II

David Lozano

Dexter Massoletti

Michael McGlynn

Robert Miller

Marco Monroy
Kenneth Osborne

Robert Purvis

Jan Pusina

Robert Richter

Catherine Siero

Robert Stevenson

Harvey Syversrud

Marsh Tekawa

Raymond Thatcher

Malcom Williams

Michael Wolfe

Administrative Support

Personnel

Erica Atkin-Orvis

Augustine Aitkens

Sue Bowen

Kenneth Bregger

Shelly Butler

Martha Condon

Richard Dicely

Cynthia Fike

Lucy Finch

Sharon Fujimura

Robert Jahnigen

Rita Jones

Joy Kono

Jacquline McDonald

Marcie Mara-Ann

Cheryl McFate

Darlene Moretti

Diana Morris

CathyPereira

Izetti Perry

Romy Perry

William Scharff

Betty Strausbaugh

Nancy Talcott

Barbara Thibadeau

Alline Tidwell

Gladys Ureta

Gregory Vierra

Olivia Wong

Katherine Williams ${ }^{\dagger}$

Judy Zelver

\footnotetext{
* Department of Physics, UCB

+ Retired
} 


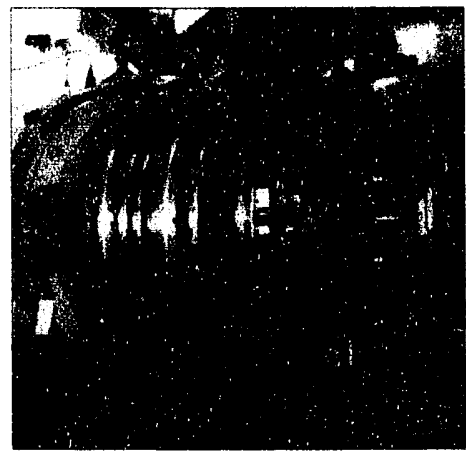

\section{AFRD: Diversity with a Common Theme}

$\mathbf{T}$

HE ACCELERATOR AND FUSION RESEARCH DIVISION is not only the largest scientific division at LBL, but also one of the most diverse. Major efforts include

- Investigations in both magnetic and inertial fusion energy.

- Design, construction, and commissioning of the Advanced Light Source, a state-of-the-art synchrotron-radiation facility.

- Theoretical and applied studies of accelerator physics.

- Research and development in superconducting magnets for accelerators and other scientific and industrial applications.

- Operation of a heavy-ion accelerator complex, the Bevalac, for nuclear science and biomedical research.

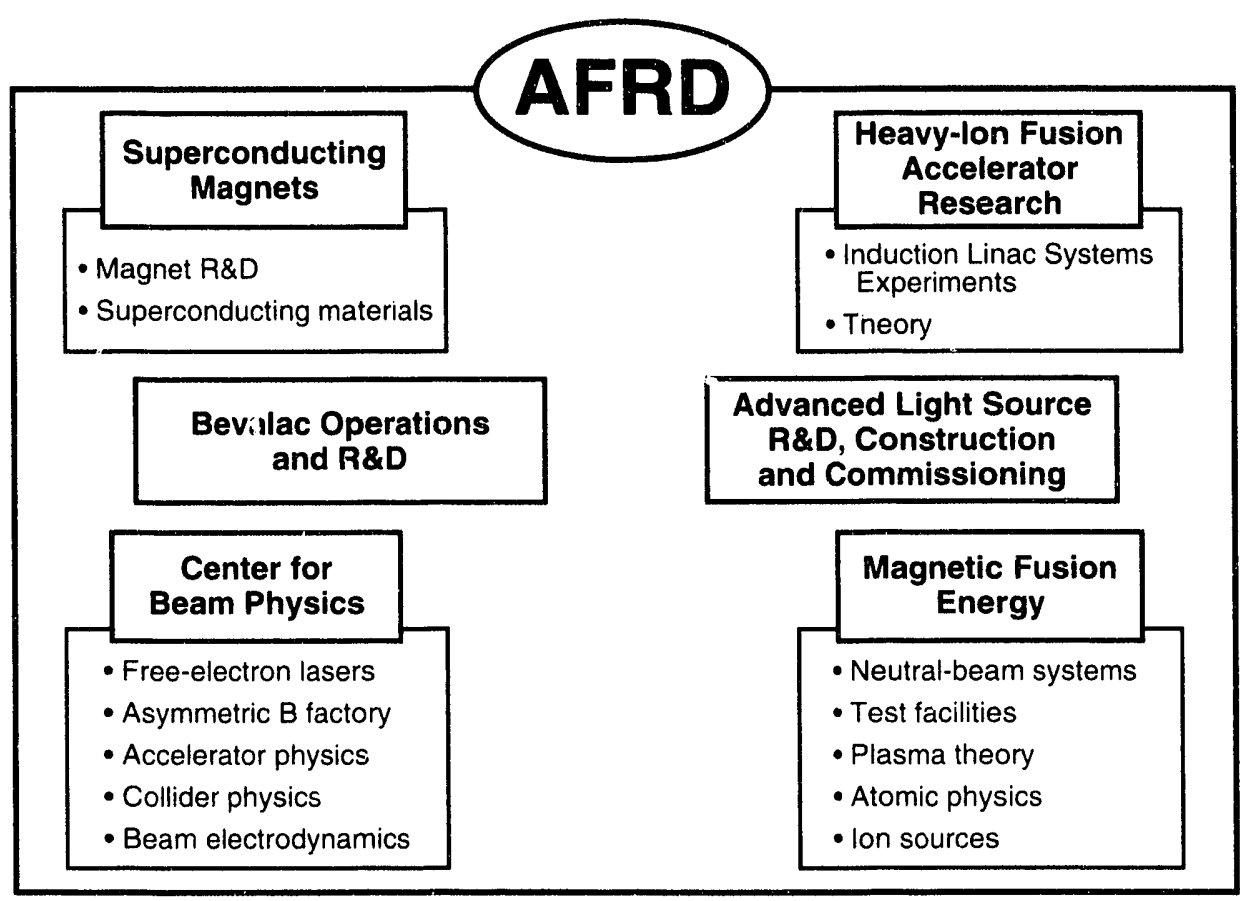

XBL 9212-5857 
These efforts share a foundation in the physics and technology of beams of ions, electrons, and photons. This introductory section gives an overview of AFRD's fields of inquiry and their relevance to current issues in science and technology. Later chapters go into greater detail on each topic.

As the industrialized world contemplates its dwindling fossil-fuel supplies and the environmental costs of energy production, nuclear fusion looks ever more appealing. Potentially one of the most efficient of all physical processes that release energy, it is also attractive from a pragmatic viewpoint. The fuel (the hydrogen isotopes deuterium and tritium) could be readily obtained, and the reactions would not leave the long-lived, highly radioactive waste products associated with fission. But controlled, self-sustaining fusion on a power-plant scale remains decades away.

The work being done today addresses two fundamental problems. One is how best to get the reaction started; a temperature of about 100 million degrees Celsius is required before random thermal interactions force the nuclei close enough to each other to fuse. (The nuclei are all positively charged and therefore repel each other; to make them fuse when they meet, the temperature must be kept high, meaning that they move fast and collide hard.) The other problem is how to make the product of density and confinement time must reach a very high value known as the Lawson criterion. Researchers in AFRD are organized into two groups corresponding to different basic approaches to these problems.

Magnetic fusion, the more familiar scheme, uses a magnetic field of great strength and rigorously maintained geometry to confine a continuously reacting plasma and keep it away from the reactor walls. In the largest of today's tokamak reactors, magnetically confined deuterium plasmas (and, in one experiment at the Joint European Torus, a deuterium-tritium plasma) have been heated to temperatures at which fusion reactions took place. The best of these brief "shots" have released about $80 \%$ as much energy as was required to heat the plasma, or about $10 \%$ of the energy that would be needed for ignition (self-sustaining fusion). In the tokamak projects, which tend to be very large and, increasingly, international, Lawrence Berkeley Laboratory has played a major supporting role. The effort focuses on development of neutral-beam injector systems that pump large quantities of energetic hydrogen or deuterium atoms into a tokamak, thereby helping to heat the already-hot plasma to thermonuclear temperatures.

This presents interesting scientific and engineering challenges: the atoms must initially be ionized, or given a charge, so they can be accelerated, but then they must be neutralized so they can penetrate the tokamak's magnetic field. AFRD's Magnetic Fusion Energy (MFE) Program supports magnetic-fusion experiments by developing ion sources, accelerators, and neutralizers. The group developed the Common Long-Pulse Source, a standard neutral-beam plasma-heater for U.S. fusion experiments, and released it into commercial production in the mid-1980s. They are now following up that achievement by designing ion sources and accelerators for next-generation tokamaks such as the proposed International Thermonuclear Experimental Reactor (ITER). To maintain larger plasmas at higher temperatures for longer periods, the neutral-beam systems that may be required in these reactors will have to be based on a different technical concept. The designs of a proposed proof-of-principle accelerator and of a test facility for it are being refined by the group.
Fusion Energy:

Beginning the Next Explorations on Two Frontiers 
The other approach is inertial fusion energy, which begins not with a plasma but with a spherical target of deuterium-tritium fuel. The target is hit from many directions at once with beams of laser light or energetic particles.

This energy bombardment heats and compresses the target enough to induce fusion; the reaction is over so quickly that the balance of forces from all sides is enough to provide containment and satisfy the Lawson criterion. The process would be repeated several times per second in a power plant.

Heavy ions (as opposed to lasers or lighter ions) appear to be the best candidates for the repetition rate, reliability, and efficiency that would be needed in a power plant. In AFRD, the Heavy Ion Fusion Accelerator

Research (HIFAR) Program theoretically and experimentally evaluates the possi-

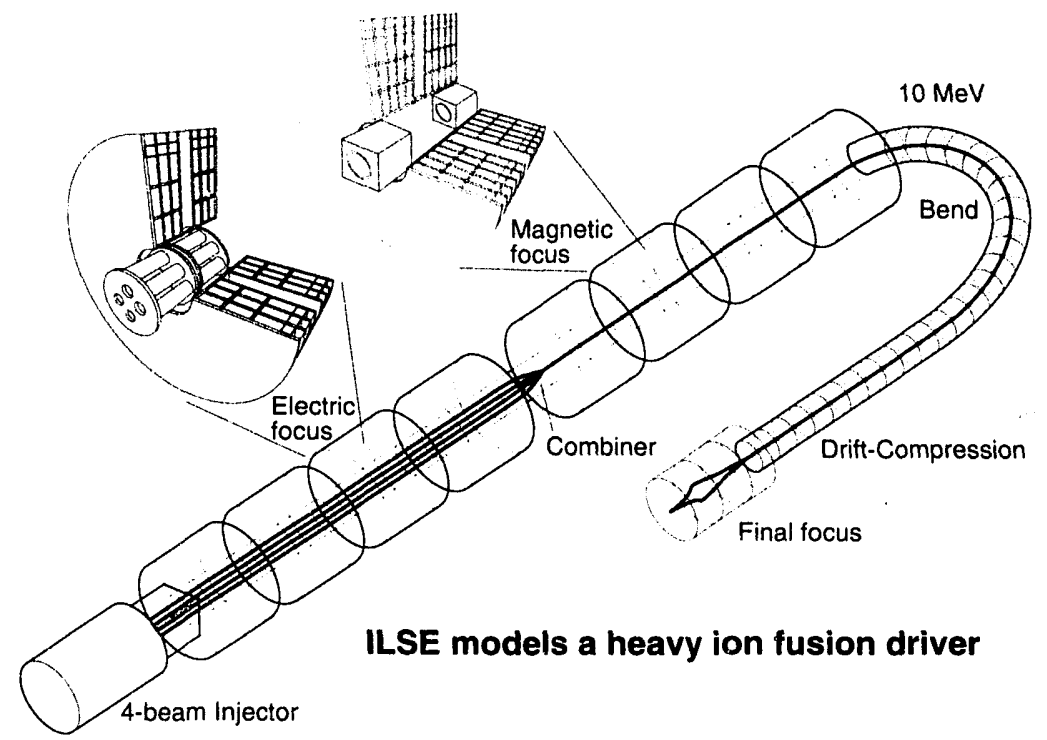
bilities for using heavy-ion beams as drivers for inertial-confinement fusion.

Since 1982, they have been progressively scaling up systems that transport and accelerate beams of heavy ions. The group recently concluded a multiyear experimental program with MBE-4, a four-beam induction linac designed to provide basic data on beam control from which information useful in the design of a fusion driver can be extrapolated. The next step, now in the engineering design phase and about to be proposed for a 1995 construction start, is ILSE, the Induction Linac Systems Experiments. ILSE will contribute further knowledge toward the goal of a full-scale driver.

XBL $931-4714$

The population of the world and the per-capita energy demand are both growing, so a shortfall is expected sometime in the next century. New sources of energy, such as fusion, will be needed to fill the gap. It will take time to meet the technical challenges of developing these new sources, so early and vigorous $R \& D$ would be prudent; therefore the National Energy Strategy calls for a fusion demonstration in the 2025 time frame. The HIFAR Program's ILSE apparatus and the MFE Program's negative-ion accelerator and test facility for ITER neutral-beam injection are among AFRD's proposed contributions to the attempt to harness fusion power. Other programs involving AFRD can also contribute directly or indirectly to alleviating the energy shortfall; an example is the proposed Chemical Dynamics Research Laboratory, with its implications for combustion efficiency. (Energy-shortfall graph courtesy LLNL)

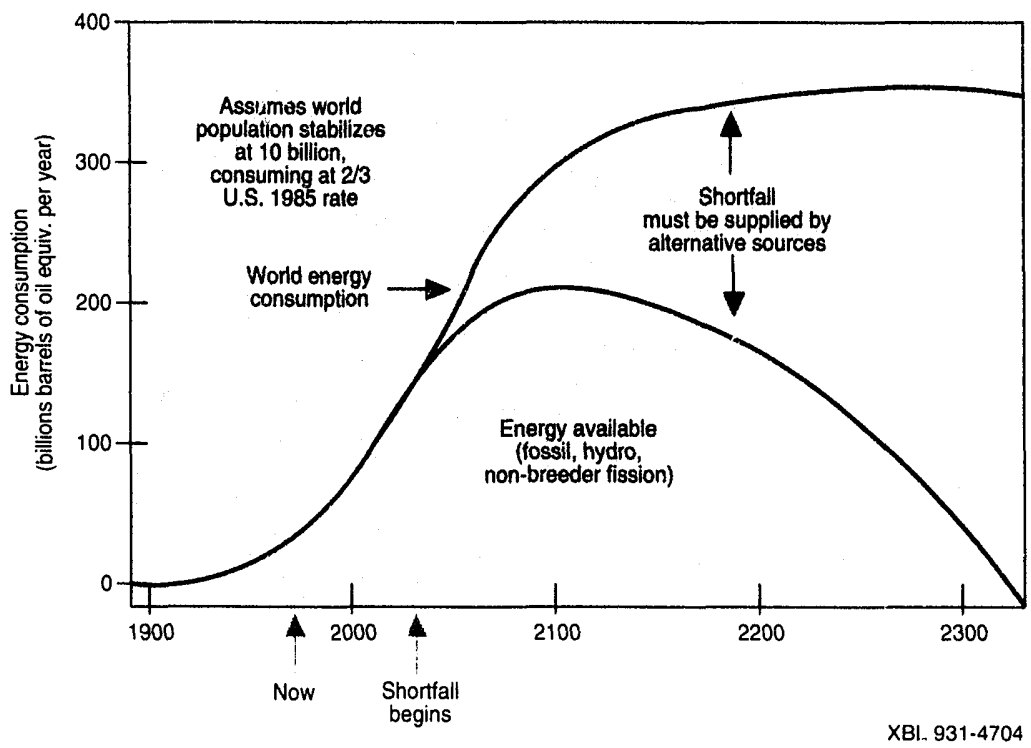


The discovery of the x-ray in 1895 revolutionized not only the work of physicians, but also that of physicists. In two decades of excitement that helped set the stage for today's knowledge of the atom, they studied the interaction of $x$-rays and matter. The results and the investigators-Roentgen, Compton, Laue, the Braggs-are familiar from freshman physics and from the roll of Nobel laureates.

After that heady beginning, the scientific and industrial uses of x-rays continued to progress, growing very subtle and sophisticated. Nonetheless, a backlog of interesting and potentially useful x-ray work began to accumulate, including studies of processes at interfaces and surfaces; microscopy and holography; and the probing of chemical reactions. The conventional means of producing $x$-rays, which involves striking a material with a beam of electrons, could generate tremendous power, but the backlogged ideas needed qualities other than sheer power: tiny, intense beams, perhaps of just one precise "color," perhaps coherent, almost like a laser beam.

The solution was found in the late 1940s in what seemed to be a completely unrelated realm: the electron synchrotron. When a magnetic field makes an electron beam change direction, photons are given off. This effect was at first considered a nuisance for robbing power from the beam and heating the accelerator and the experimental apparatus. But beginning in the 1950s, scientists began to realize that this nuisance had desirable qualitiesthat $x$-rays of unparalleled intensity could be obtained. The pioneers of synchrotron-radiation work obtained this radiation "parasitically" from electron accelerators meant for high-energy physics.

In the 1970s, there appeared a second generation of synchrotronradiation sources: a generation of electron storage rings ${ }^{*}$ whose reason for existence was the production of synchrotron light. AFRD advanced this new field by developing practical versions of magnetic devices called "wigglers" and "undulators" that could further manipulate an electron beam, producing radiation with selectable bandwidth and coherence.

During the early and mid-1980s, AFRD began designing a third-generation synchrotron-radiation facility. The hallmarks of the third generation are high-quality electron beams (small source diameter and low transverse energy), along with a ring design that lends itself, both mechanically and in terms of maintaining beam quality, to the insertion of numerous wigglers and undulators. In 1986, the groups within the Advanced Light Source project officially began the detailed design of the ALS, a national user facility that is expected (presuming upon a successful Operational Readiness Review) to be fully commissioned and ready for users in 1993. By the end of fiscal 1992, the staged construction and commissioning activities were nearing a major milestone: injection of an electron beam into the storage ring. Meanwhile, work continued on design and fabrication of insertion devices and beamlines. Development of the user program continued, with LBL and outside researchers designing experiments and equipment to take advantage of this bright new source as they enter the second century of the $x$ ray.

\footnotetext{
"In a storage ring, a variation on the general theme of the synchrotron, a large number of accelerated particle bunches go around a "racetrack" millions of iimes for repeated re-use, as opposed to being delivered once to a fixed target.
}

Advanced Light Source: Seeing the Future in a New Light 
The multiple straight sections in the ALS can each accommodate a magnetic insertion device to enhance synchrotron-light production. The bending magnets in the arc sections (shown during an f'arly phase of installation in late 1991) produce useful synchrotron radiation as well. The high-brightness beams of ultraviolet and soft-x-ray light from the ALS are of interest to a wide variety of researchers in fields ranging from structural biology to the fabrication of next-generation computer chips.

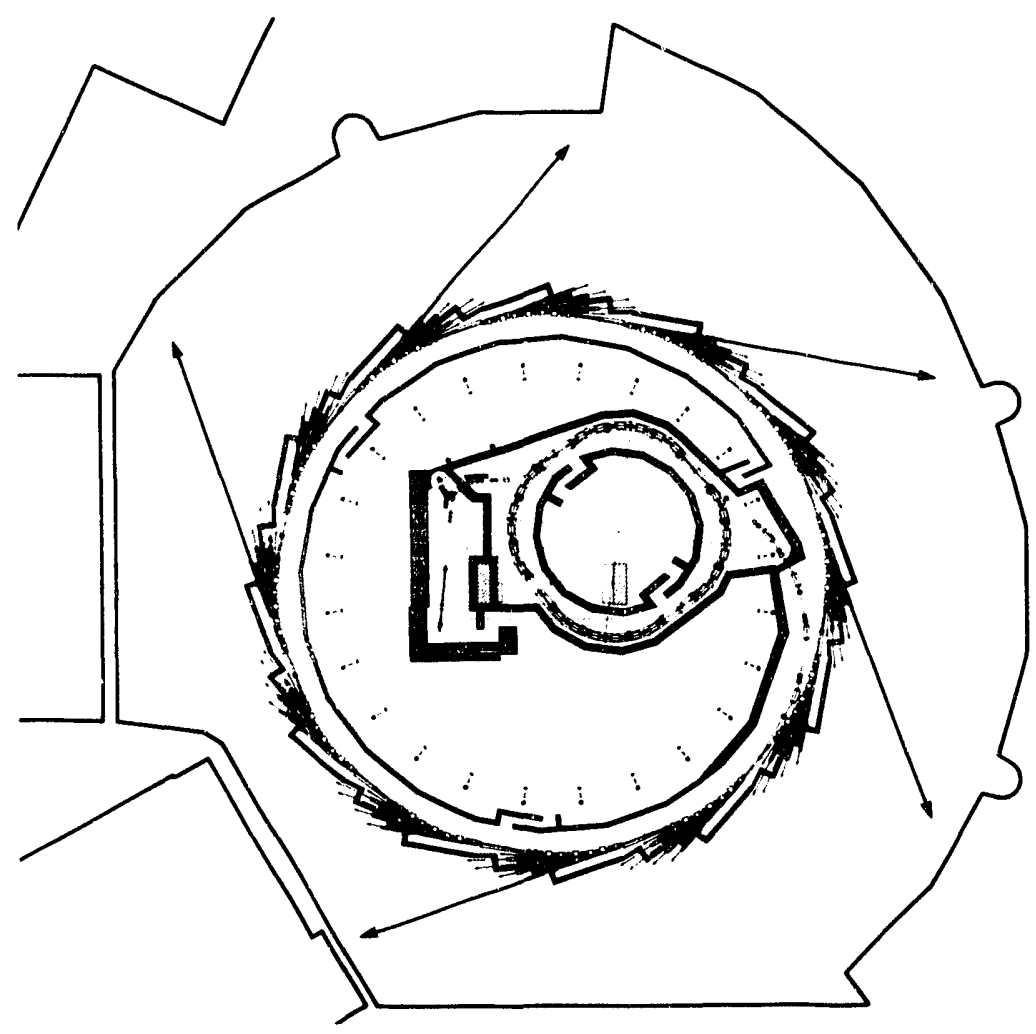

XBL $881-8810$

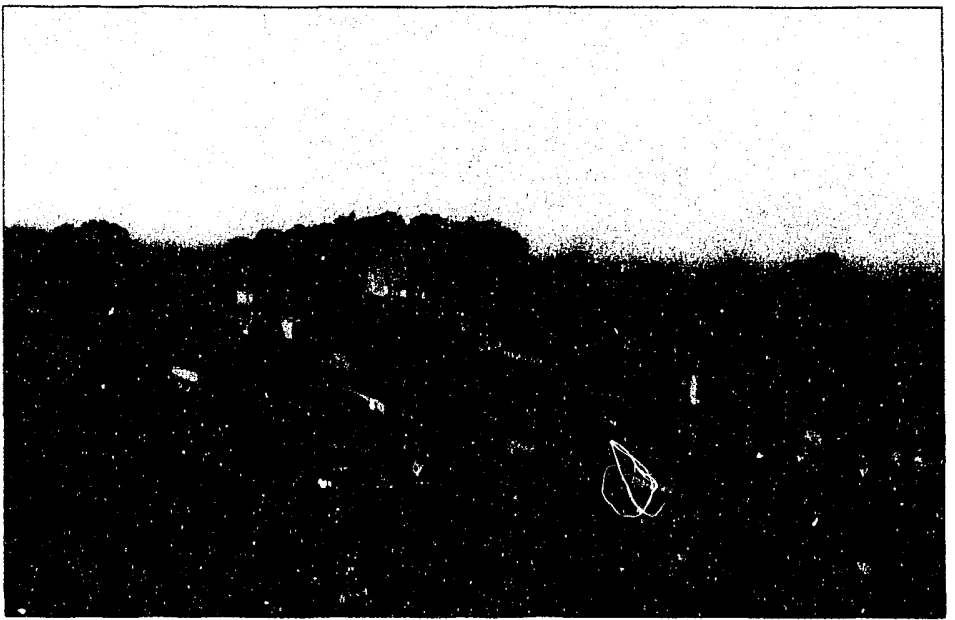

XBC 912.709

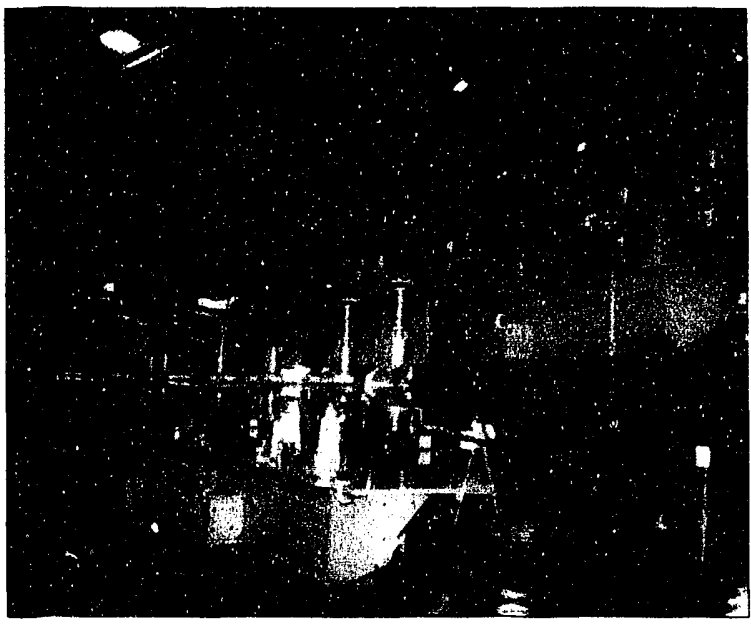

XBC 917-5682 
The Superconducting Super Collider, the most ambitious of particle accelerator projects, will have a pair of underground storage rings, 52 miles in circumference, in which protons circulate in opposite directions. The two beams, each with an energy of approximately $20 \mathrm{TeV}$, will cross and collide in several "interaction halls." High-energy physicists need such energies to put hypotheses about the basic structure of matter to experimental test. SSC energies should be the hunting grounds for particles that have been postulated but never observed and measured, such as the top quark and the Higgs boson. Perhaps its users will even find particles, phenomena, or parameters that do not fit today's theories-an exciting prospect that has been a hallmark of accelerator-based physics.

The design and construction of such a large facility have occupied researchers from many laboratories. AFRD's Superconducting Magnet Program has played a key role in the SSC effort for several years, concentrating on the design and manufacture of the superconducting wire and the cable made from it, and on design and testing of the quadrupole magnets used in the collider-ring lattice. These magnets have to meet exacting performance specifications, especially in terms of magnetic-field uniformity. They must also be extremely reliable in order to give users the high experimental statistics needed at the frontiers of high-energy physics. Much effort has gone into optimizing seemingly minute design details that would affect performance under those conditions. Anticipating the need for private industry to mass-produce the SSC's thousands of magnets, we embarked upon a Magnet Industrialization Program to transfer the technology for building them. In 1991 and 1992, engineers from Babcock and Wilcox and from Siemens worked alongside us to build collider quadrupoles.

While working in support of the SSC's immediate needs, we have also looked toward the future. With one of our experimental high-field dipole magnets, D19, we set a world record for field strength in accelerator-style magnets: $10.06 \mathrm{~T}$ centrally and $10.4 \mathrm{~T}$ near the side of the bore. We also began work on D20, an accelerator-style magnet that uses a different type of superconductor (which requires innovative construction techniques) and should achieve fields considerably greater. Other work included cablingmachine development, which anticipates requirements for different cable designs in our program. We also continued exploring the science and technology of superconducting materials and magnets in many other ways. Our ongoing materials program is working with private industry on superconductor that incorporates "artificial pinning centers" for better performance, and is also involved in development of "high-temperature" superconductors into magnetic materials that are useful in a macroscopic engineering sense. Better ways of designing accelerator magnets are also of interest, as are potential nonaccelerator uses of high-field magnets, such as nuclear magnetic resonance equipment. The program is rooted in the technology of advanced accelerators, a community that it continues to serve, but its work also has the potential for direct and indirect payoff in several other fields that could benefit from cheaper and better superconducting materials.
Magnets for the SSC and Beyond 


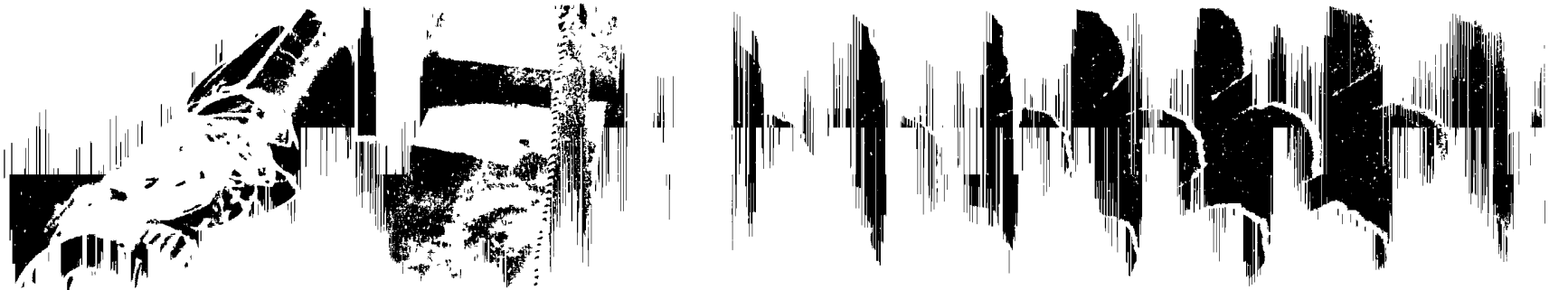


Th': Center for Beam Physics* is a key element in several of the division's diverse activities, assisting with immediate programmatic needs and-as indicated by the Carl Sandburg quotation at right-also laying foundations for future research. A highlight of the program's 1992 accomplishments was the design of the Beam Physics Facility. This new facility that makes double use of the ALS injection linac during the long periods when the ALS storage ring is full and the injection complex is therefore idle. In one of the two experiments currently planned, the $50-\mathrm{MeV}$ electron beam will be crossed with a visible laser beam in an attempt to produce femtosecond $x$-ray bursts through $90^{\circ}$ Compton scattering. The other experiment will test new ideas for plasma focusing of an electron beam.

One of the Center's areas of involvement is the proposed Chemical Dynamics Research Laboratory, a new initiative by LBL's Chemical Sciences Division that is designed for synergy with the ALS. In 1992, the Center's researchers refined their concept for an infrared free-electron laser (IRFEL) that features tunability, high power, and fine resolution. By combining the IRFEL's powerful, tunable output with beams available from the ALS, chemical lasers, and molecular-beam? sources, the CDRL could offer unprecedented opportunities for studying pure and applied reaction dynamics and a variety of topics in materials and surface science. In this new design, the proposed IRFEL uses superconducting accelerating cavities to achieve greater energy stability in the electron beam (and thus in the photon beam) and to achieve continuous-wave rather than pulsed operation.

High-energy physics also figured prominently in this divisional center's efforts. Another major initiative, which is being spearheaded by the Stanford Linear Accelerator Center with collaboration of LBL and Lawrence Livermore National Laboratory, is PEP-II, an energy-asymmetric B-meson "factory" based on the existing PEP storage ring at the Stanford Linear Accelerator Center. Creating B mesons and their antiparticles in electron-positron collisions that have a moving center of mass, an idea originated by LBL Deputy Director Pier Oddone, will spatially separate the decay products, making detection simpler than it would be if the center of mass were fixed. This will enable high-energy physicists to study charge-parity violation and rare $\mathrm{B}$-meson decays.

The Collider Physics group within the Center continued collaborating in basic and applied research for the generation of high-energy accelerators beyond the SSC. Their efforts focused on a futuristic electron linac called the two-beam accelerator, driven by misrowave power from either a freeelectron laser or a relativistic klystron. Other contributions to high-energy accelerators come from the Beam Electrodynamics Group. Their research emphasizes electromagnetic analysis and various radiofrequency "gymnastics" to ensure proper beam behavior-a set of skills that have contributed to a variety of projects in recent years, including the ALS and the Tevatron, and are now being brought to bear on the $B$ factory.
Center for Beam Physics: "Nothing Happens Unless First a Dream"

*Formerly the Exploratory Studies Group. 
The United States currently gets more than $90 \%$ of its total energy from combustion, so even small improvements in combustion efficiency could have tremendous benefits. The proposed CDRL is part of the Combustion Dynamics Initiative, a joint undertaking with sites at $L B L$ and Sandia National Laboratories in Livermore, CA. The CDRL will offer an unprecedented opportunity to bring together various technologies for probing energetic, transient chemical reactions at a very fine level of detail. The community of potential users is putting together a broad program of pure and applied studies, ranging from reaction dynamics to applied work that might ultimately help increase engine efficiency or reduce air pollution. Shown here is the spectral coverage that would be available to CDRL users with the proposed superconducting IRFEL.

\section{CDF/LBL Technical Facilities}

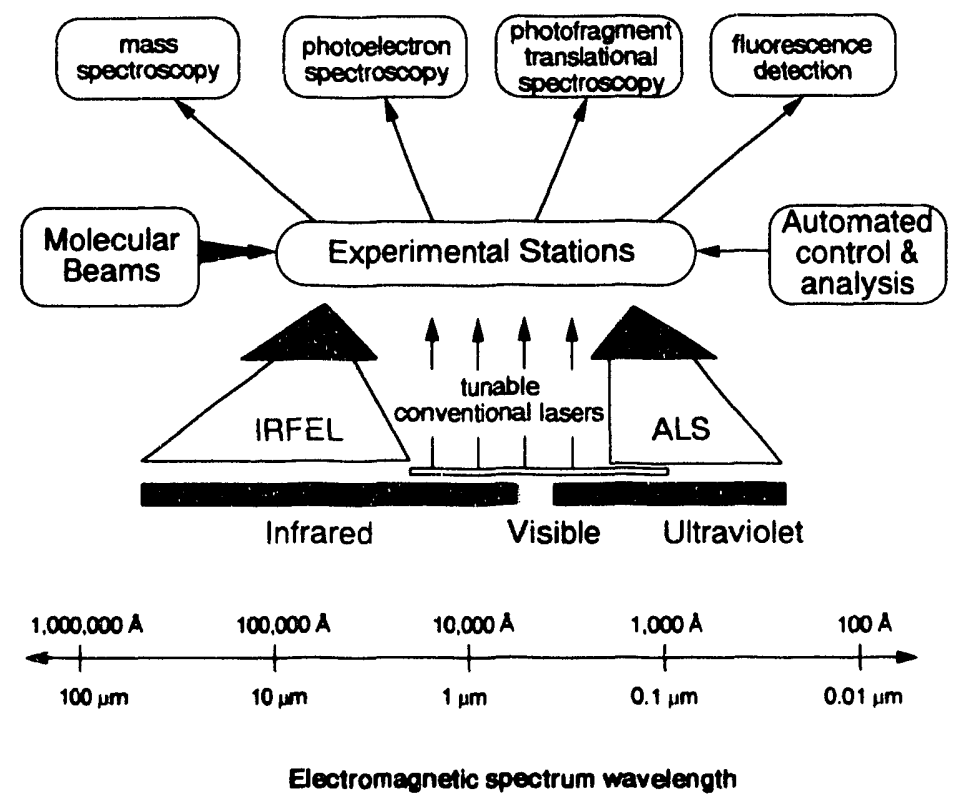

\section{U.S. ENERGY CONSUMPTION}

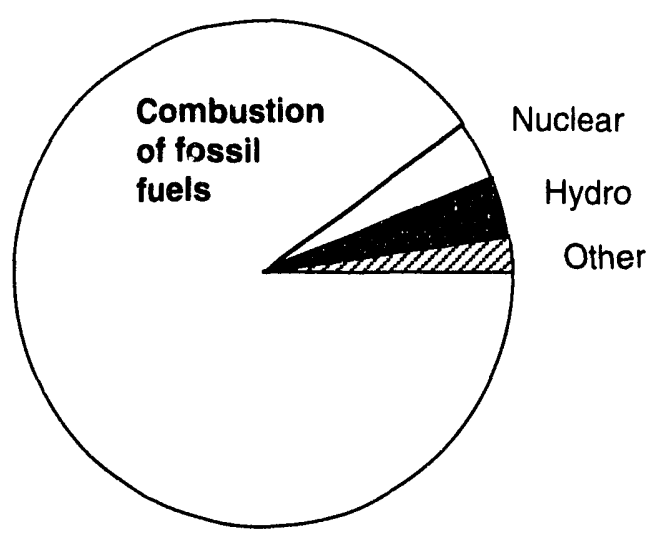

This picture will not change appreciably in the foreseeable future

XBL. $9112-6886$

CBB 905-3635 
After nearly four decades of service, which resulted in scientific productivity not even dreamed of by its original builders (and which entailed many an ingenious upgrade), operations at the system of two accelerators known as the Bevalac are expected to stand down for the last time in early 1993.

The older of the two, the Bevatron, was the most energetic proton synchrotron of the early 1950s. It was there that Segrè and Chamberlain discovered the antiproton, an achievement that won them the Nobel Prize. After two decades of contribution to the "particle explosion" that revolutionized subatomic physics, the accelerator was thought to be nearing the end of its career, but thanks to an imaginative idea, some of its brightest days were still ahead. The idea was to feed the Bevatron with the beam from the nearby SuperHILAC heavy-ion linear accelerator, which itself was well known as the discovery tool for several transuranic elements. When the SuperHILAC-to-Bevatron transport line was completed in 1974, the result was a unique system that could accelerate heavy nuclei to $\mathrm{GeV}$-per-nucleon energies. An early-80s upgrade called the Uranium Beams project, which included a third ion injector at the SuperHILAC, a new vacuum liner in the Bevatron, and a computerized control system, enabled acceleration of any naturally occurring element. In 1983 the Bevalac set a total-energy record, which still stands, by accelerating uranium to $960 \mathrm{MeV} / \mathrm{n}$.

This high-energy, heavy-ion capability gave rise to the "Bevalac era" in nuclear science - an era of great advancement in science and technical discovery, an era when the behavior of nuclear matter at extremes of temperature and pressure first came under concentrated scrutiny. Biomedical research and treatment of patients with particle beams, another field pioneered at LBL, also became an important part of the Bevalac's programs.

The Bevalac-era interest in nuclear matter under extreme conditions has engendered a variety of programs elsewhere, including a major new initiative at Brookhaven National Laboratory called the Relativistic Heavy-Ion Collider. Charged-particle radiotherapy is also beginning to come into its own-a privately funded proton facility at the Loma Linda University Medical Center recently joined several governmentally sponsored efforts worldwide. Ironically, the Bevalac itself could be supported no longer in an era of tightly constrained research budgets.

The Bevalac will live on in a variety of ways. Spinoff technologies from the constant effort to use and improve the Bevalac have included novel ion sources, beam-delivery systems for medical treatment, dosimetry technologies, and an easier-to-make version of an accelerator called a radiofrequency quadrupole. Three generations of scientists came to the Bevalac to study the nature of matter and the behavior of irradiated cells. Most important, the Bevalac will live on through its contributions to knowledge: numerous individual discoveries that, when taken together, compose a theme of finding the unexpected. This has been a leitmotif of accelerator-based physics, and is an auspicious legacy for the machines and experiments of today and tomorrow that owe so much to the Bevalac.
The Long Shadow of the "Bevalac Era" 
An aerial photograph, with the beam path superimposed, shows how a geographical coincidence inspired a singularly successful idea. The Bevalac's combination of energies and ion species have ensured it a long and scientifically productive life. The 39-year-old Bevatron and the 35-year-old SuperHILAC, separately and together, have been tremendously productive to science. Some of the findings have been quite unexpected and extremely important (nuclear antimatter, strange particles, and resonances at the Bevittron, five manmade elements at the HILAC and SuperHILAC, and nuclear compressibility at the Bevalac, to name a few). Hundreds of LBL and visiting researchers have worked at these machines, and more than $\mathbf{8 0}$ students have written doctoral dissertations on related research. The challenge now is to identify facilities and funding for the vast amount of work that has yet to be done.

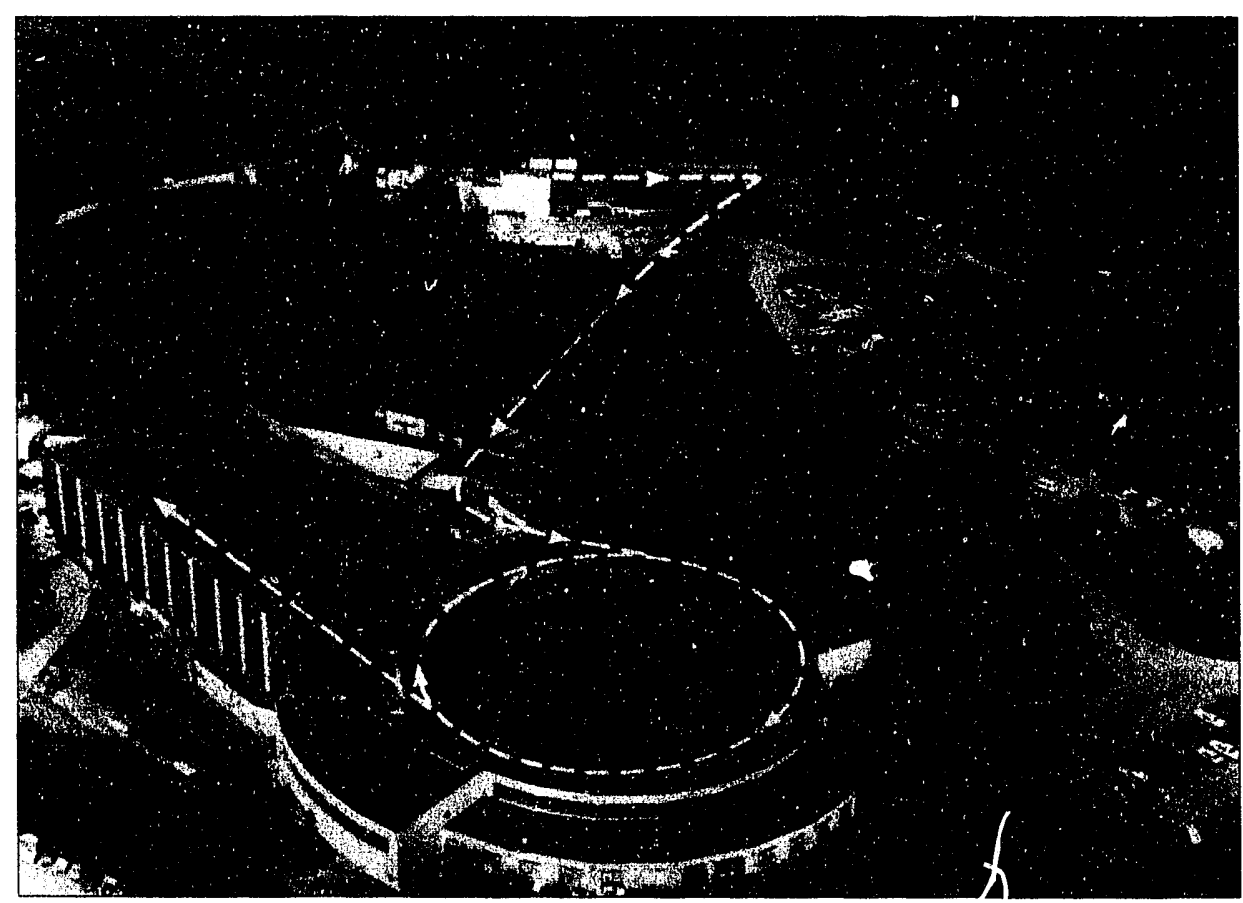

XBB 766-4673A 


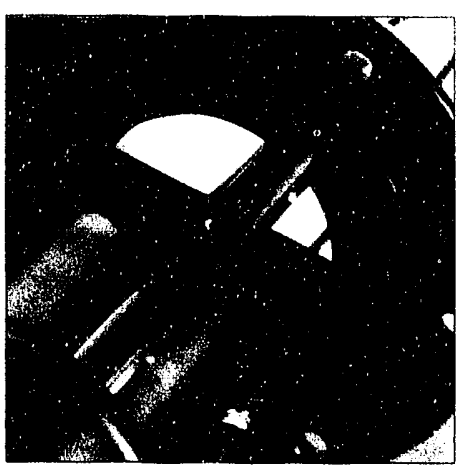

1.

\section{HEAVY-ION FUSION} ACCELERATOR RESEARCH

$\mathbf{T}$

HE HEAVY-ION FUSION ACCELERATOR RESEARCH (HIFAR) program in AFRD has the long-range goal of developing accelerators for fusionenergy production. Heavy-ion fusion, like laser fusion, uses intense beams to implode and ignite, or "drive," small targets containing thermonuclear fuel. This creates a burst of energy that can be contained in a target chamber or reactor. The beams from the driver (a particle accelerator or laser) are focused onto the target, located at the center of the chamber, by lenses outside the chamber. The targets typically would have a radius of a few millimeters and the target chamber would have a radius of a few meters. In this scheme, called inertial fusion energy (IFE), the fuel "burns" in pulses that take place so rapidly the reactants are confined by their own inertia. This may be contrasted with the other main approach to fusion, magnetic fusion energy (MFE), in which the fuel is confined by magnetic fields and burns in long pulses or continuously.

The National Energy Strategy, published by the Department of Energy in 1992, calls for a demonstration IFE power plant by the year 2025. The cornerstone of the plan to meet this ambitious goal is research and development for heavy-ion driver technology. A series of successes indicates that the technology being studied by the HIFAR Group-the induction accelerator-is a prime candidate for further technology development toward this long-range goal.

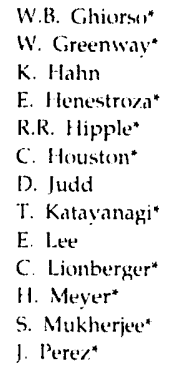

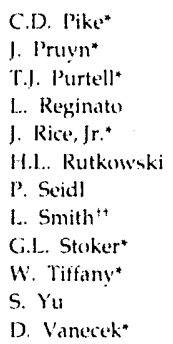

Sludents
N. Barboza
A. Brown
C. Friesen
J. Kung
N. Nathwani
D. Keeney
K. Reach
Administrative Support
S. Bowen
D. Norris
A. Tidwell
O. Wong

\footnotetext{
* Engineering Division

"Lawrence Livermore National Laboratory

Retired
} 
The emphasis on heavy-ion drivers is readily understandable. For engineering and economic feasibility, drivers must be both reliable and efficient. They must also have a high pulse repetition rate (several pulses per second) and long life (about 30 years). Existing drivers--lasers and light-ion accelerators-are excellent for near-term research, but they have been designed for a low repetition rate, typically a few shots per day. Therufore, new drivers must be developed for power production. During the last decade, nearly all high-level DOE and Congressionally mandated committiees have identified heavy-ion accelerators as the most promising drivers for actual power plants.

The HIFAR program addresses the generation of high-power, highbrightness beams of heavy ions; the understanding of the scaling laws that apply in this hitherto little-explored physics regime; and the validation of new, potentially more economical accelerator strategies. (The strategy-validation issue is especially important because an inertial-fusion power plant will have to be a success not only in physics and engineering, but also in commerce.) Key specific elements to be addressed include:

- Fundamental physical limits of transverse and longitudinal beam quality.

- Development of induction modules for accelerators, along with multiplebeam hardware, at reasonable cost.

- Acceleration of multiple beams, merging of the beams, and amplification of current without significant dilution of beam quality.

- Final bunching, transport, and focusing onto a small target.

In 1992, the HIFAR Program was concerned principally with the next step toward a driver: the design of ILSE, the Induction Linac Systems Experiments. ILSE will address most of the remaining beam-control and beammanipulation issues at partial driver scale. A few parameters-most importantly, the line charge density and consequently the size of the ILSE beamswill be at full driver scale. This will allow us to model some particularly important beam behaviors, which were not addressed by the earlier experiments, at energies much lower than those of a driver. The ILSE proposal was well received, and the DOE responded with a Determination of Need, the first "key decision" on the way to authorization of a construction start.

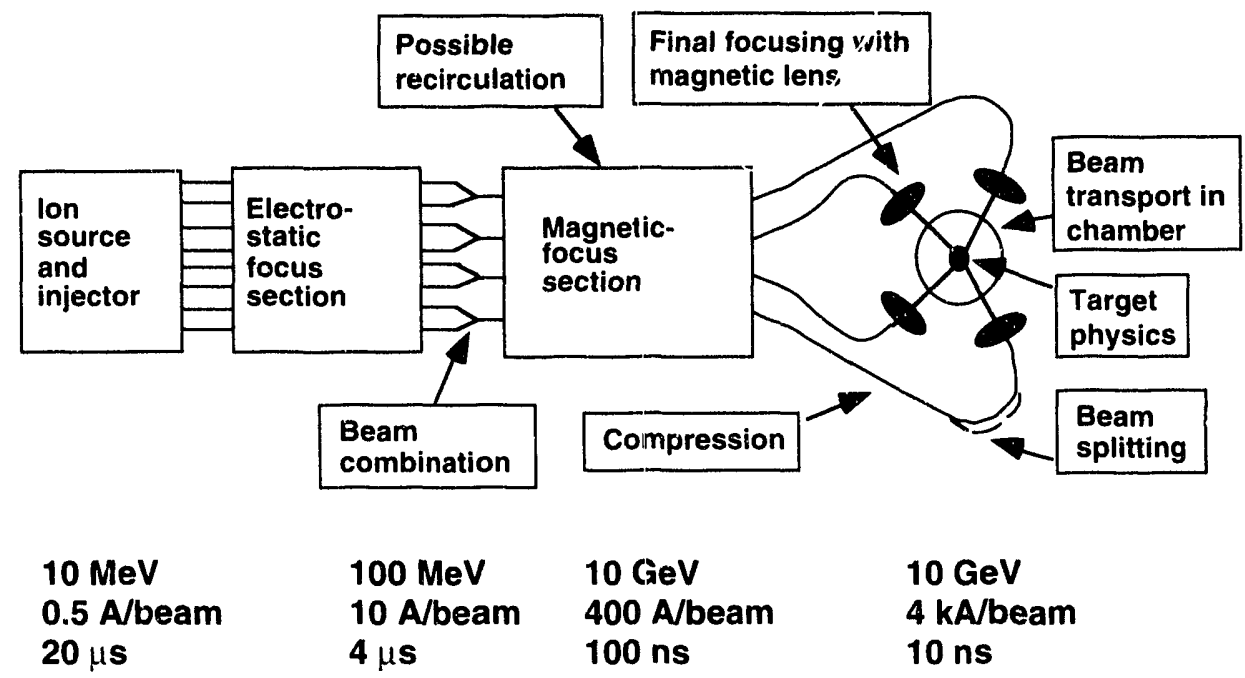

Figure 1-1. The accelerator systems and beam manipulations found in typical heavy-ion driver designs are represented by boxes. A highly accurate alignment system, not shown, will be used throughout. The shaded boxes represent systems that have been tested in past experiments (AccSys Technologies is planning a small-scale focusing experiment on LBL's existing SBTE apparatus). The remaining issues, except target physics, will be tested in ILSE and its anticipated experimental program. Typical driver values of energy, current, and pulse length are shown at various stages. 


\section{Induction Linac Systems Experiments}

\author{
ILSE Physics Design
}

A theory group closely integrated with the experimental groups continues supporting present-day work and looking ahead toward larger experiments and the eventual driver. Much of the grolip's effort in both areas during 1992 was of course focused on ILSE, but long-range theoretical and experimental research continued. Highlights of this long-range, driver-oriented research included continued investigations of longitudinal instability and some new insights into scaled experiments with which we might examine hard-to-calculate beam-dynamics phenomena.

The next logical step on the road to a driver is ILSE, the Indution Linac Systems Experiments. The multi-heam apparatus will provide the first data on several significant capabilities that will be necessary or at least economically desirable in a driver. They include:

- Combining parallel ion beams dominated by space charge.

- Making the transition from an electrostatic to a mugnetic beam-transport system.

- Magnetic bending of intense, space-charge-dominated ion beams.

- Amplifying current by "drift compression."

- Focusing ion beams precisely onto a small spot.

A new design for the appuratus was developed in 1992, and a Conceptual Design Report, or proposal, was prepared in April. The design has since been undergoing improvements and will be proposed in fiscal year 1993 for a construction start in FY95.

In the ILSE conceptual design (Figure 1-2), four beams are accelerated and electrostatically focused, then combined into one, thus providing data on a possible driver feature." The beams are further accelerated in a subsequent section, this time with magnetic focusing. In both the electrostatic-focus and the magnetic-focus sections, the beam is compressed-another important feature. Then the beam is used for experiments in drift compression and final focus.

The new ILSE also has the same line charge density, in beampipes of the same diameter, as the electrostatically focused section of a postulated driver. ILSE will thus allow us to test this driver parameter at full scale. (Most ILSE parameters, as shown in Table 1-1, are scaled down from those of a driver.) Another aspect of ILSE that may turn out to be highly relevant to a driver is a set of provisions for recirculating acceleration.

Economic studies of recirculating induction ion accelerators, performed at Lawrence Livermore National Laboratory (LLNL) in collaboration with our group, have indicated that such a driver might be less expensive than a linear induction accelerator. However, much less is known about the physics of recirculating induction ion accelerators. Various schemes are being

\footnotetext{
"Beam combination appears to be a good way to increase the current of the beams without greatly increasing the length of the accelerator (which, in a driver, will be some hundreds of meters). This would be economically attractive. However, it would not be strictly necessary should experiments show that it is not feasible to combine driverlike beams without spoiling the beam quality.
} 


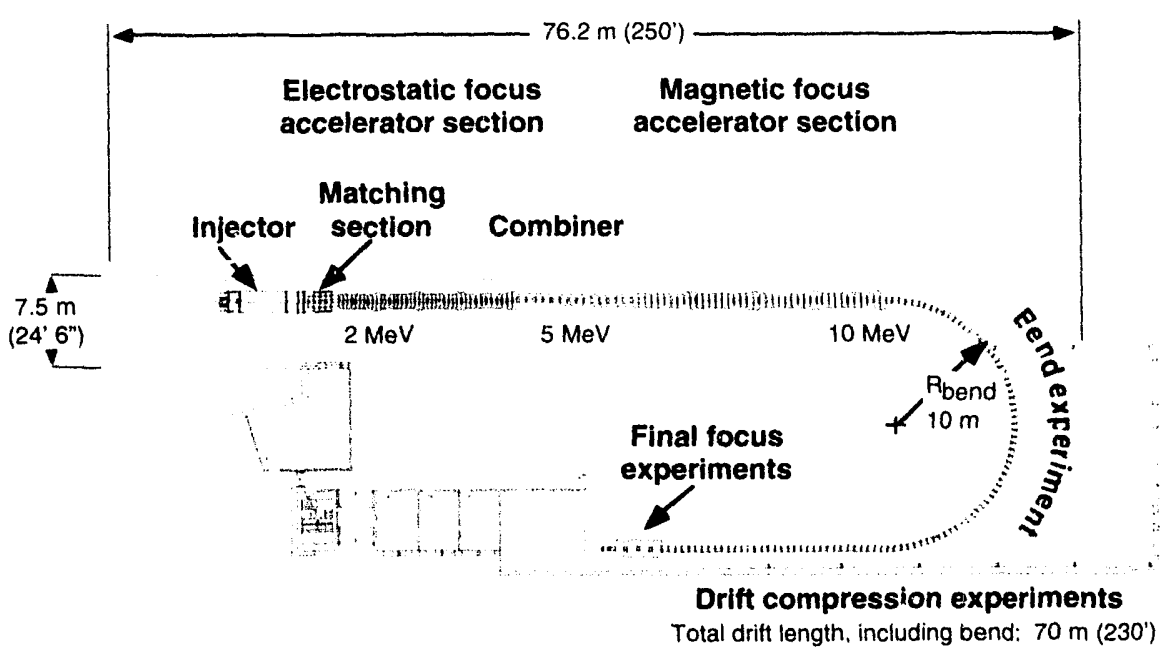

XBL $931-4711$
Figure 1-2. This diagram shows the physics design of the ILSE accelerator and a possible arrangement of some of the experiments. The 2-MeV injector provides four beams of $\mathrm{Ne}^{+}$or $\mathrm{K}^{+}$in 1$\mu$ s pulses at driver line-charge densities. A matching section transforms the round beams to an alternating gradient profile and "squeezes" them together for insertion into the electrostatically focused linac. To amplify the current, the electrostatic-focus induction linac imparts a velocity shear or "tilt" to the beams as it accelerates them to $4.5 \mathrm{MV}$. A subsequent magnetic-focus induction linac, which also amplifies current, takes one of the beams to $10 \mathrm{MeV}$. A later phase of ILSE will combine the four beams into one for injection into the magnetic-focus linac.

studied for extending the ILSE sequence of experiments to cover the essentials of the recirculating approach in case theoretical work indicates the desirability of these experiments.

The result of the physics design effort is a "point design" - a selfconsistent design, selected from a broad continuum of possibilities, that best addresses our experimental needs within our space and funding prospects. The engineering design that builds upon the physics point design was completed in 1992 and is being refined to best fulfill our experimental needs within realistic funding prospects.

ILSE will be constructed in two phases. During the initial "project" phase, the electrostatic-focus section (which accelerates the beams from 2 to about $5 \mathrm{MeV}$ ) and the later magnetic-focus section ( 5 to $10 \mathrm{MeV}$ ) will be connected by a transfer line. The transfer line will contain diagnostics and

Table 1-1. Key parameters of HIFAR experiments and an example driver.

\begin{tabular}{lcccc} 
& SBTE & MBE-4 & ILSE & $\begin{array}{c}\text { LMF-based } \\
\text { example }\end{array}$ \\
\hline Ion species & $\mathrm{Cs}^{+}$ & $\mathrm{Cs}^{+}$ & $\mathrm{K}^{+}$ & $\mathrm{Kr}^{+}$ \\
Number of beams & 1 & 4 & $4 \rightarrow 1$ & $20 \rightarrow 4^{+}$ \\
Final voltage $(\mathrm{MV})$ & 0.15 & 1 & 10 & 25000 \\
Total final energy $(\mathrm{J})$ & 0.07 & 0.08 & 60 & $5 \times 10^{\circ}$ \\
Final ion velocity/c & 0.0016 & 0.004 & 0.03 & 0.25 \\
Bunch length $(\mathrm{m})$ & 8.0 & $1.1 \rightarrow 0.25$ & $8.8 \rightarrow 8.8^{+}$ & $70 \rightarrow 10^{+}$ \\
Pulse width $(\mu \mathrm{s})$ & 20 & $2 \rightarrow 0.4$ & $2 \rightarrow 1^{+}$ & $24 \rightarrow 0.1^{+}$ \\
\hline
\end{tabular}

* These pulse lengths are given at the end of the accelerator section. In a driver, drift compression would further shorten the pulse. Note also that smaller, less-intense beams can be compressed further.

t The bunch lengths and pulse widths specified here, with their beam-compression implications, are hypothetical driver values, not calculated LMF values. 
will introduce an offset that couples one of the four beams into the magneticfocus section. During this phase, the other three beams will not be accelerated in the magnetic-focus section.

Later, during the experimental program, we will remove the transfer line to provide space for combiner experiments that merge the four beams to

make one beam of higher current.

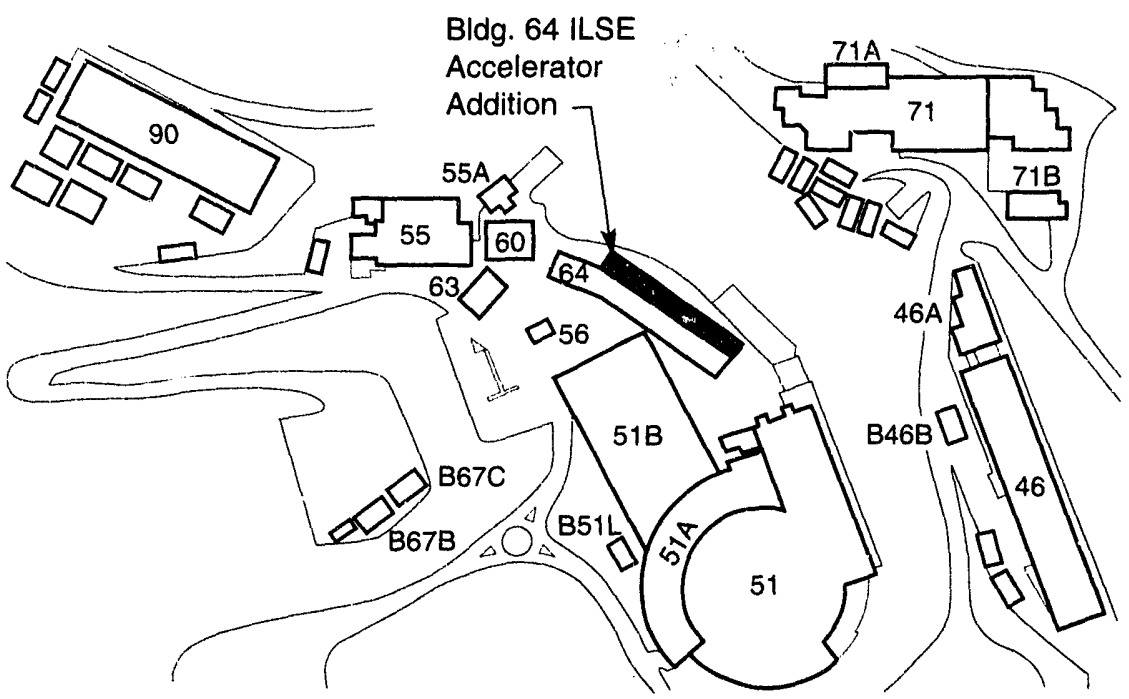

The magnetic-focus accelerator will continue current amplification " while accelerating the beam to $10 \mathrm{MV}$. Construction would start in fiscal 1995 under the proposal that we plan to submit in 1993; various parts of the experimental program would follow successive intermediate stages of construction. A variety of siting options are being evaluated, a decision process that is being coordinated with the probable decommissioning of the Bevatron. Figure 1-3 shows the option that was described in the 1992 Conceptual Design Report,

XBL 9212-5855

Figure 1-3. The 1992 proposal specified an ILSE site in is an area behind Building 64 near the Bevatron accelerator. ILSE would be housed largely in a new twostory addition to Building 64 . Other possibilities are being examined, including part of the site of the Bevatron itself, which was recently scheduled for shutdown and decommissioning.

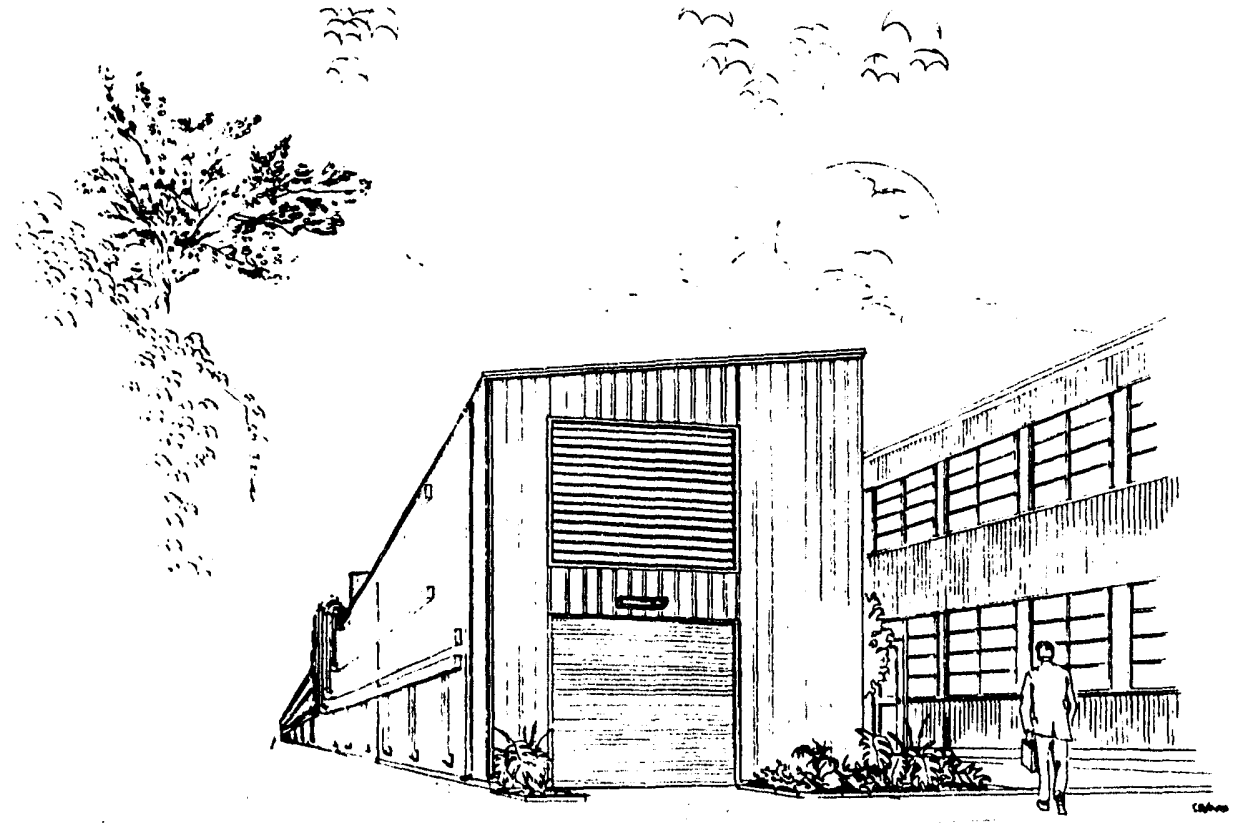

XBL $931-4712$

\footnotetext{
"Current amplification, an important driver feature, is accomplished by tailoring the accelerating pulses in order to impart a "velocity tilt," or make the particles in the rear of a bunch go faster than the ones in the front. This presents a number of challenges in technology and operations, but its basic feasibility was demonstrated in our earlier MBE-4 experiment. In the "experiment" phase of ILSE, we may test drift compression after the accelerator-another driver feature in which the last acceleration modules give the tail of the bunch one last kick, creating a velocity tilt that has its beam-compression effect on the way to the target.
} 
which was written before the rews of the Bevatron's 1993 closure was received: an addition behind Building 64 near the Bevatron. The External Particle Beam hall (user area) of the Bevatron now looks like the most attractive site: it is a large high-bay building with infrastructure items of suitable capacity, such as electrical utilities.

A broad R\&D program is being conducted to develop ILSE components and systems, including the ion source and injector, the induction cores, and beam instrumentation. Theoretical work has also been done on the methods and effects of transverse beam combination in ILSE and in a driver. A conceptual design study of the ILSE combiner is in progress.

Progress toward an injection system for ILSE continued on several fronts in 1992. An existing carbon-arc source and $1-\mathrm{MeV}$ injector were used for various experiments with transient $\mathrm{C}^{+}$pulses. A study group examined possible approaches to the injection system, recommending a 1-MV electrostatic aperture accelerator feeding an electrostatic-quadrupole accelerator. Subsequently a scaled experiment was performed on the SBTE, Single-Beam Transport Experiment (the principal HIFAR apparatus from the early 1980s, which has been brought out of mothballs several times for beam-transport and focusing studies). Meanwhile, development and evaluation of various candidate ion sources continued; it now looks as though we will use a hotsurface alkali-metal source. We also began design work on a major modification of the 2-MV Marx generator; the new design, which will not have to supply long pulses, should offer much lower impedance.

The study group, composed of personnel from LBL and Lawrence Livermore National Laboratory (which has collaborated with us extensively on ILSE), along with other experts, was established to study the injection needs of ILSE. Information was gathered from other laboratories that have built high-voltage injectors; this was followed by a large theory effort to model the candidate injectors in two and three dimensions. Two candidates were examined: the electrostatic aperture column (ESAC) acceleratorwhich in our case would be a diode, a single-gap accelerator, operated at 0.5 to $1 \mathrm{MV}$-and the electrostatic-quadrupole-focused column accelerator (ESQ). Each proved to have its strengths and weaknesses. The ESAC approach entailed increased risk of insulator breakdown as the total voltage increased. The ESQ could cause emittance growth because of the "energy effect," a phenomenon in which different energies are imparted to particles at different radial positions in the quadrupole apertures even if their axial positions are the same.

The study culminated in a design workshop that recommended a combination of the two approaches, each in its most appropriate role: an 0.5-1 MeV ESAC feeding an ESQ that takes the beam to the full injection energy of $2 \mathrm{MeV}$. This technology is similar in concept to an accelerator being developed by AFRD's Magnetic Fusion Energy group, which has collaborated with us extensively during the ion-source and injector efforts. We plan to build a single-beam prototype in 1993.

The injector will obtain $\mathrm{K}^{+}$from a surface-conversion ion source in which $\mathrm{K}$ or another alkali metal is embedded in an aluminosilicate (zeolite) carrier. (Zeolite can be infused with a great deal of carried material, which gives it a variety of industrial and scientific uses; in this case, the benefit is long source lifetime.) An injector with an ESAC first stage might require a source with a diameter of about $22.4 \mathrm{~cm}$ and a current density of about $2 \mathrm{~mA} / \mathrm{cm}^{2}$. Zeolite Ion-Source and Injector
Development 
sources can definitely meet the ILSE requirements, which are a total current of about $790 \mathrm{~mA}$ of $\mathrm{K}^{+}$with a normalized transverse emittance of a fractional mm-mrad. After considerable applied research, we have developed a new potassium-implantation technique that combines long life and the required performance: tests of a 2.5-cm-diameter prototype (Figure 1-4) on SBTE indicated a current density of $19.5 \mathrm{~mA} / \mathrm{cm}^{2}$ (limited by the optics of the extraction diode, not by the source's capabilities), a normalized transverse emittance of $0.2 \mathrm{~mm}$-mrad, and a lifetime of some

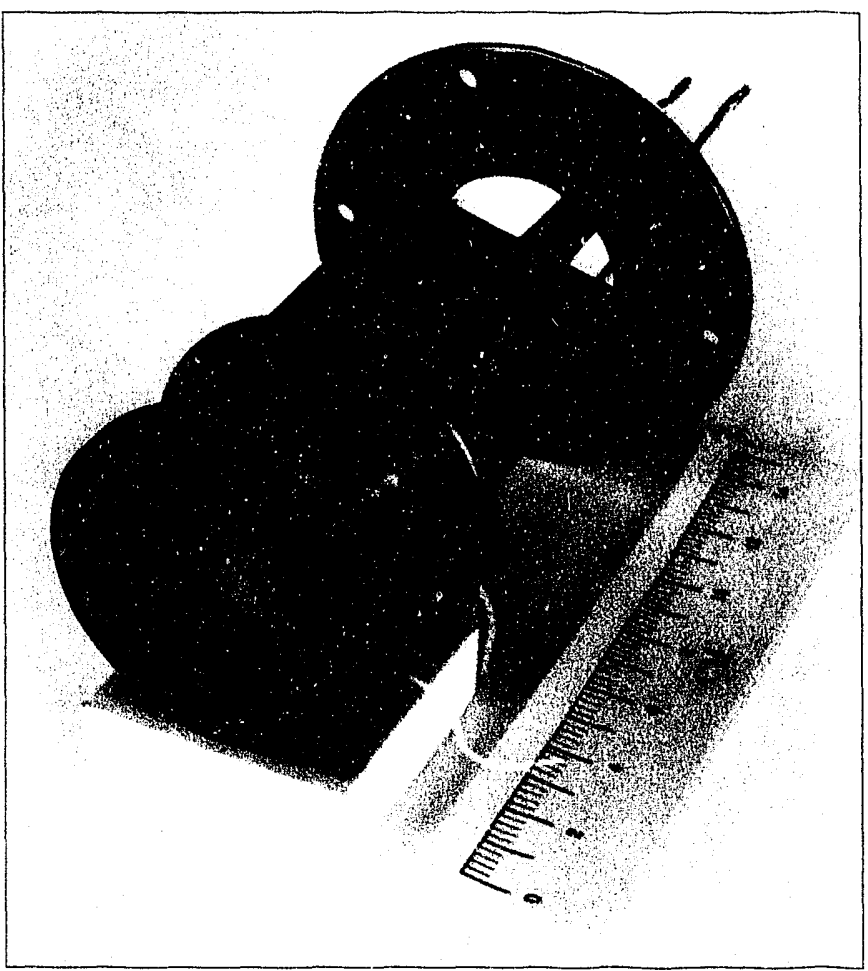
months in the ILSE duty cycle. We are now extending R\&D efforts to sources with diameters in the 10 cm range.

Another approach that was studied for possible use in ILSE was an rf-powered source in which the plasma is confined by a multicusp magnetic field, much like the ion sources developed by AFRD's magnetic fusion energy program and described in Chapter 2 of the AFRD summary of Activities. The low ion temperatures reported for these sources should permit development of flexible, low-emittance sources suitable for our needs. The higher line charge density of these beams also supports the goal of modeling some characteristics of a driver. Finally, these sources can provide a variety of ion species, including various noble gases, which is interesting because ILSE can be operated with ion species ranging in mass from carbon to potassium.

CBB 920-9404

Figure 1-4. This one-inch experimental ion source incorporates two LBL-developed improvements to an existing technology: artificial zeolites incorporating the desired ion species, which in this case is potassium. The two innovations are applying the zeolite to a porous tungsten plate and heat-treating it between 1400 and $1700^{\circ} \mathrm{C}$. In this range, crystals a fraction of a millimeter in length, similar to the mineral cristobalite, are formed. Lower temperatures, as in previous attempts to use zeolite, sinter the material, compromising its mechanical integrity, and higher temperatures would vitrify it. The use of porous tungsten helps keep the zeolite coating from puddling into nonuniformities on the curved surface, a defect that would show up in the extraction optics, and also contributes to mechanical strength. The result is a source that meets ILSE's severe requirements for low beam "temperature" and shows no physical or performance degradation in one month of simulated ILSE duty. Shown here are the zeolite in its heat shield, as well as a complete ion source in Pierce geometry.

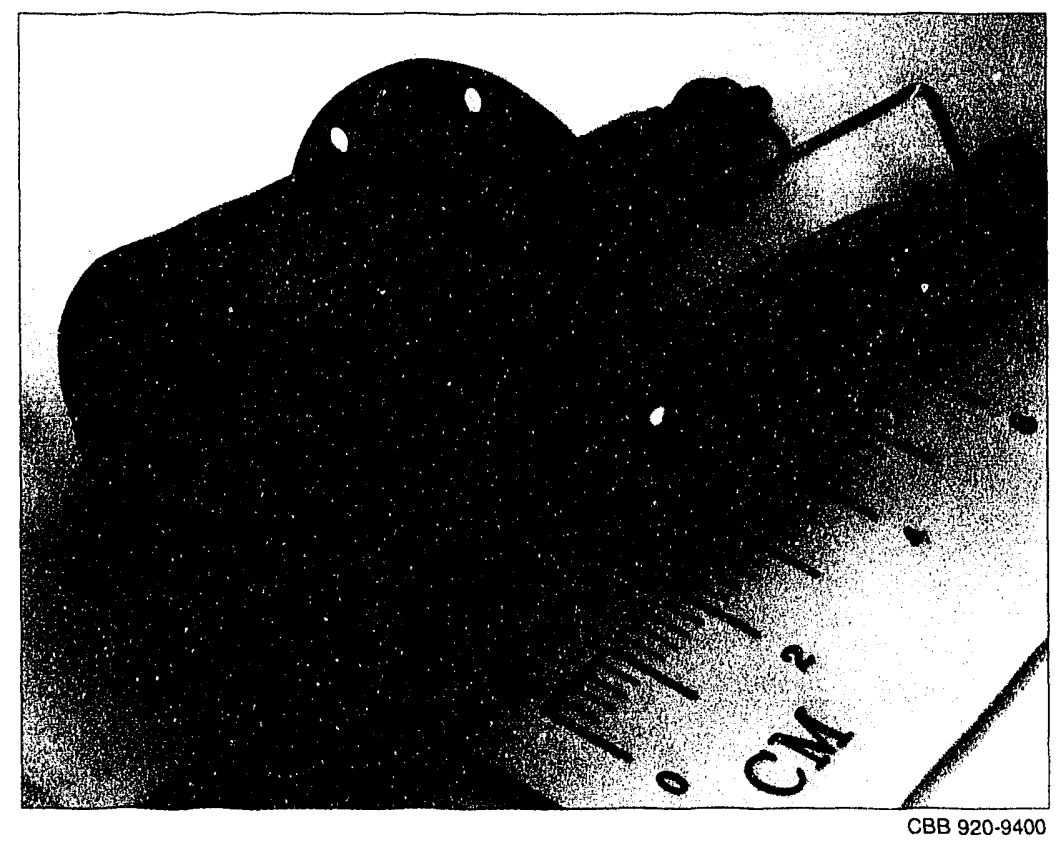


The choice of ion species is a complex issue (sidebar); the 1991 physics point design assumed that ILSE would accelerate $\mathrm{Ne}^{+}$. However, the new point design uses $\mathrm{K}^{+}$, a species readily available from the zeolite source, and the extraction of short pulses from plasma sources (which were designed as part of a program that deals primarily with long pulses) can be problematic. (If the use of small extraction apertures proved feasible, we could use higher ion temperatures and therefore obtain shorter pulses without compromising the source brightness.) The multicusp source remains promising as a "fallback position" or as a source of nonmetallic ion species for experiments that have not yet been conceived.

In preparation for using an ESQ stage in the ILSE injector, we have been performing an ESQ physics experiment on SBTE. The apparatus was equipped with one of the aluminosilicate $\mathrm{K}^{+}$ion sources, and the accelerating Studies on SBTE column was modified into a single diode with a $3.3-\mathrm{cm}$ gap to accelerate the beam to $27 \mathrm{kV}$. The three electrostatic quadrupoles from the SBTE matching section were turned into an accelerating ESC by reconnecting their power supplies; this ESQ accelerates the beam to $54 \mathrm{kV}$. With this configuration, we are studying the beam dynamics along the ESQ section. These experiments search for a subtle emittance growth due to the "energy effect," as mentioned earlier. This potential problem, which was predicted by various studies, including simulations with the particle-in-cell code ARGus, affects beams whose longitudinal energy is large in proportion to the quadrupole voltage and whose envelopes are large enough to approach the quadrupoles. The phase-space profile in the vertical direction, the emittance growth along the ESQ, and the amount of the beam hitting the quadrupoles were found to be consistent with the ARGus results and with Faraday-cup measurements of total beam current at various points. Similar horizontal measurements are next on the agenda. We also plan to reconfigure SBTE for a scaled injectordiode-and-ESQ experiment that will examine the transverse beam dynamics in the ESQ, and we will experiment with a quadrupole correction scheme that should eliminate the predicted growth in transverse emittance.

\section{Selecting an Ion}

ILSE could accelerate a range of elements at various charge states. Choosing the best one for the experimental program is subtle and complex.

In ILSE, the capabilities of the magnetic beam-transport section will play a major role in the decision, as they will in subsequent systems. The force that can be exerted by a given magnetic field depends on the velocity, and thus the energy, of the ion beam. The heavier the ion, the bigger and more expensive the accelerator required for a given velocity. In ILSE, a few-MeV beam of an ion species of moderate mass will allow approximation of the beam physics of a very heavy ion species at many tens of $\mathrm{MeV}$. Also important are the ease and reliability with which multi-ampere quantities of the ion can be produced in the desired charge state.

In principle, higher charge states are desirable because they multiply the force that a given electric or magnetic field can exert upon the ion. (The charge of an ion is the "handle" by which it is manipulated.) In practice, however, sources capable of higher charge states tend to put out the lower ones as well. Separating the undesired species does not appear practical for the purposes of ILSE. Another factor, more significant for future experiments than for ILSE, is that the actual beam current is likewise multiplied, possibly crossing into a region of high-current instability for the energy in question.

Subject to these considerations, $\mathrm{K}^{+}$has been chosen for the 1992 ILSE physics point design.

As heavy-ion IFE progresses to target shots, the desired power deposition in the target will become an important factor. The driving beams have to deposit roughly $10^{15} \mathrm{~W} \mathrm{~cm}^{-2}$ in the outer shell of the target over a period of about $10^{-8} \mathrm{~s}$. To achieve the correct combination of power and range, heavier ions need higher energies but correspondingly lower currents, an advantage that helps avoid high-current instabilities. Economic considerations also come into play; doubling the current would increase the risk of high-current instabilities (and the required strength of the focusing fields) but would also halve the length of the accelerator. Studies indicate that $\mathrm{Bi}$ and $\mathrm{Hg}$ - possibly in a charge state as high as +3 , with the +1 and +2 ions magnetically separated-are promising candidates. 
Induction Core R\&D
Induction-accelerator designers have a variety of magnetic materials at their disposal for the acceleration cells. In an induction accelerator for HIF, the ion current will typically be a small fraction of the magnetizing current at the beginning of the acceleration cycle. The magnetic material chosen sets the current drive required from the modulator. Hence the choice of materials is determined by the combined cost of the material and the modulator driving it, as well as by the performance requirements. The logical choice for ILSE is Metglas, a "metallic glass" magnetic alloy, available in various formulations, that is used in large volumes for $60-\mathrm{Hz}$ power applications. Because of its intrinsic properties (high resistivity and high capability to respond to timevarying magnetic-field gradients) and a method of fabrication that leads to very thin ribbon, Metglas is also well suited for the ILSE application, which requires rates of magnetization of $1-5 \mathrm{~T} / \mu \mathrm{s}$.

Some research and development was required in order to learn how the power industry's materials and practices might apply to a short-pulse induction accelerator. At $60 \mathrm{~Hz}$, the metallic glass is annealed to maximize $\Delta B$. At $60 \mathrm{~Hz}$, only a few millivolts of potential difference is induced between adjacent turns of ribbon, so surface oxides and resistivity provide sufficient insulation. However, the high magnetization rates in ILSE, the voltage induced per turn will be closer to 10 V. Coatings that could withstand. the annealing temperatures and would not adversely affect the magnetic properties are available, but would add considerably to the cost of the cores, so we began experimenting with unannealed Metglas on our induction-core test stand (Figure 1-5).
Figure 1-5. A test stand enables us to make a great many measurements, without beam but otherwise under realistic conditions, of prototype ILSE induction cores. A cross section of a typical three-core cell from the electrostatic-focus accelerator section is shown in the line drawing.

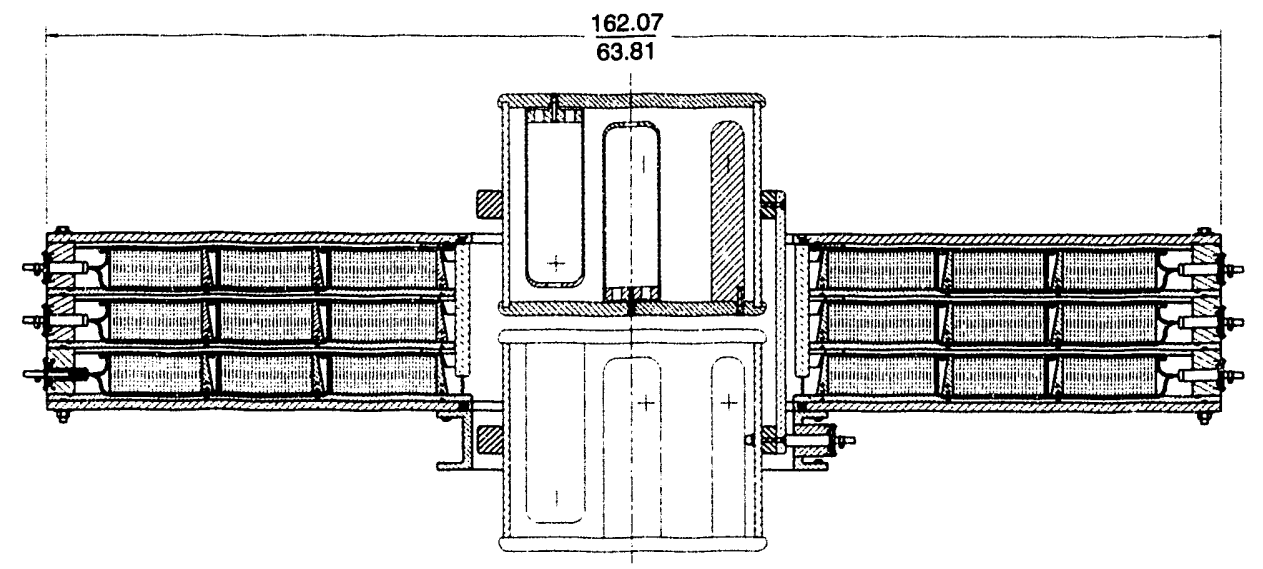

XBL $9212-5856$ 
The tests were conducted using two cores made with 2605 SC Metglas, the alloy with the optimum properties for ILSE. One core was wound by our technicians, the other by AlliedSignal Corp., the supplier of the material. The results (Figure 1-6) confirm that the unannealed material is quite acceptable for ILSE. Our 1993 plans include constructing enough cores with this optimum material to make up one $33-\mathrm{cm}$ lattice half-period of ILSE for further development of pulsed power supplies and waveform correction systems.

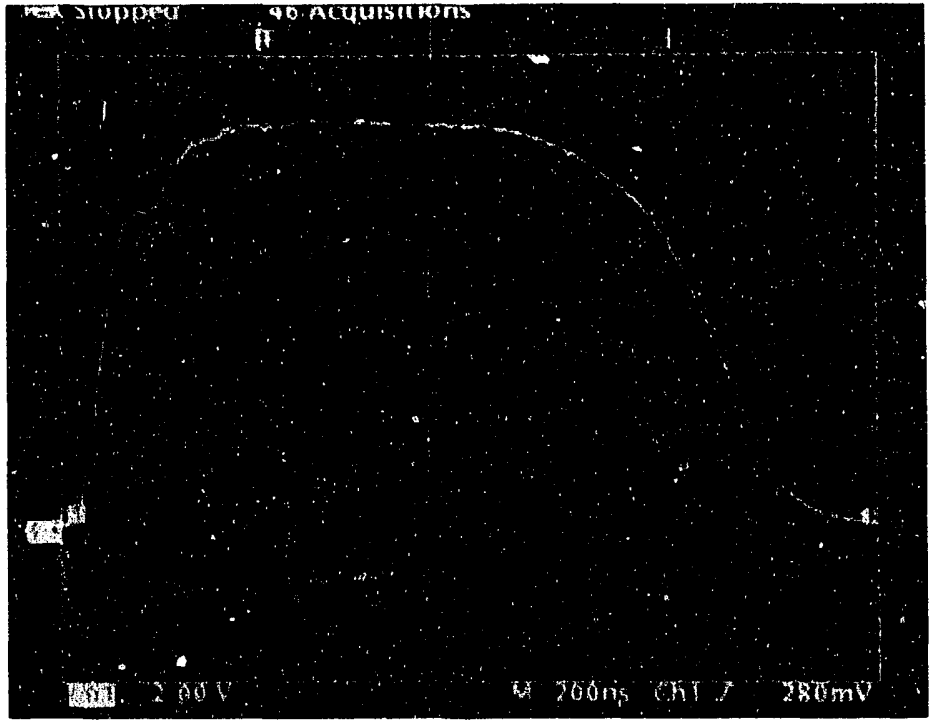

XBL $9211-2515$

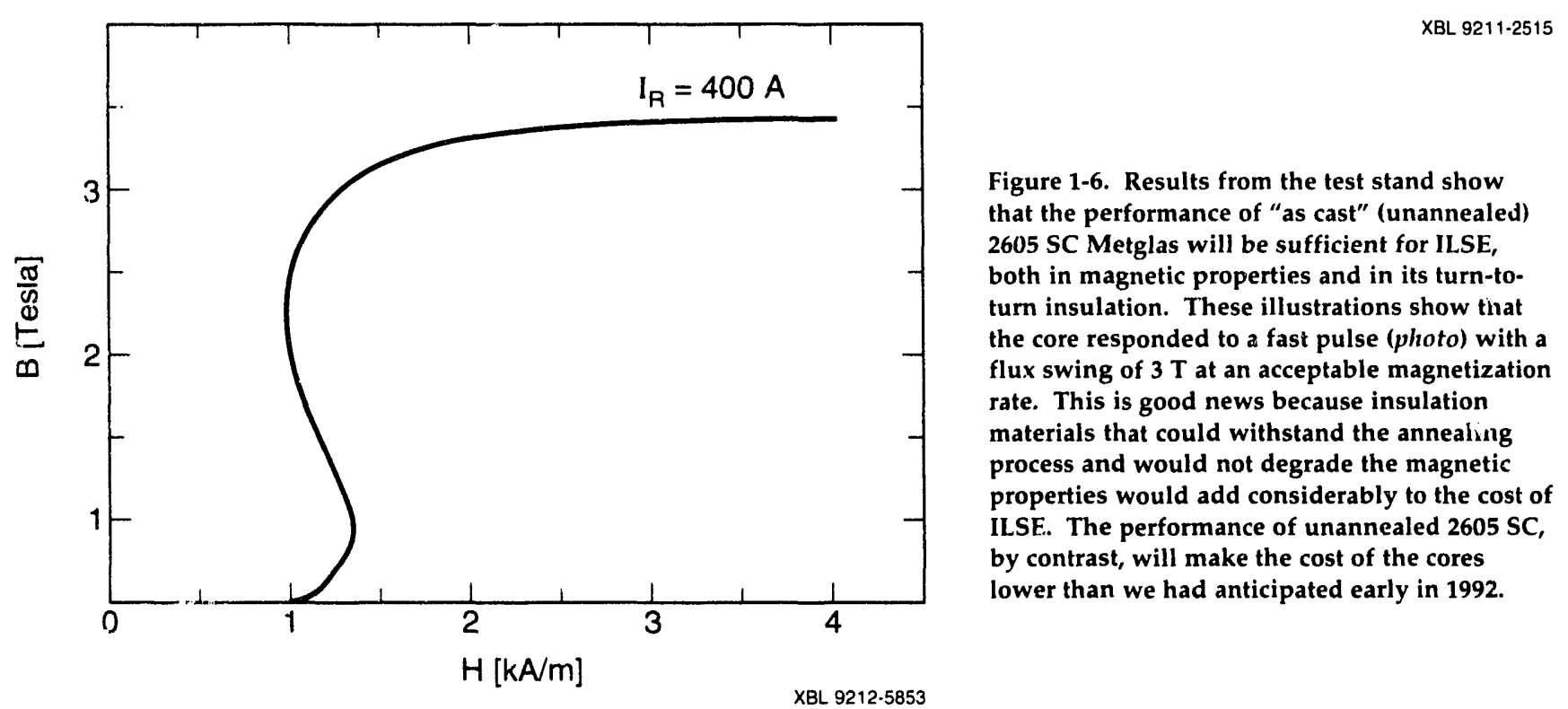

An important factor in the design of ILSE (and subsequent accelerators for Magnet Design heavy-ion fusion) is the maximum beam current that can be transported through the quadrupoles of the magnetic-focusing accelerator section. The greater the current per beam, the fewer the beams and therefore the lower the cost. Using numerical particle simulation we have studied this problem in terms of preserving beam quality during transport (that is, minimizing emittance growth) and determining the maximum dynamic aperture that can be achieved.

In 1992 our magnet design work concentrated on large magnets whose radius is a significant fraction of the accelerator's lattice half-period. In such magnets the anharrnonic "fringe fiel.$s$ " that extend longitudinally beyond 
the winding become increasingly important. As an aid in this effort, we improved our simulation code HIFI to account accurately for the way ILSE's space-charge-dominated beams* respond to nonlinear external focusing fields. One of the candidate designs that resulted has no azimuthal multipoles whatsoever apart from the desired quadrupole field.

However, the effects of higher-order components are not necessarily adverse. We began a study, using analytical methods and transport simulation codes, of the effect that nonlinear transverse and longitudinal fringe fields exert upon emittance growth. It could be that judicious introduction of an octupole field (a technique used in many final-focus magnet designs) will be able to significantly reduce the emittance growth that stems from these fringe fields.

Also underway is a study of dynamic aperture (the cross-sectional area beyond which oscillations are no longer coherent and particles are lost to nonlinear-dynamic processes) as a function of phase advance per cell and the relationship of this range to the maximum transportable current. It appears that the maximum transportable current is nearly invariant for phase advances of $10-45^{\circ}$ even though the corresponding beam size varies by more than a factor of two over this range- - a finding that promises considerable latitude in this parameter when a driver is designed.

The "LMF driver" is a point design for a heavy-ion driver that was studied on behalf of the Laboratory Microfusion Facility, a possible weapons-physics and weapons-effects facility that has been discussed in recent years. An IFE power-plant driver is expected to be similar to the LMF driver.

\section{Long-Range Research and Development}

\author{
Longitudinal \\ Instability Studies
}

Although present-day and near-future research programs such as ILSE occupy much of our attention, we also engage in various experimental and conceptual efforts (by ourselves and in collaboration with colleagues from other institutions such as LLNL) that look further dozn the road to a driver.

Studies have indicated that a driver of the kind we are studying - an induction linac accelerating high-current pulses of heavy ions at subrelativistic velocities-may be subject to a slow-growing instability: the unstable growth of current fluctuations. The unstable mode is caused when the beam inciuces an electromotive force in the induction cores of the accelerator; this "back-EMF" affects the beam. The net effect is a growing wave that moves forward in the accelerator but does so more slowly than the beam and thus moves backward in each pulse. This effect is closely related to various phenomena that have to be avoided or controlled in other accelerators, such as the transverse beam-breakup mode in electron linacs and an instability that affects cold beams of either ions or electrons in storage rings.

In the late 1980s, as driver concepts became better defined, we and others realized that the drivers' parameters, including the use of multiple beams, pushed the predicted growth length of the instability into the $50-500 \mathrm{~m}$

\footnotetext{
"The ion beams in a driver and in ILSE are strongly space-charge-dominated rather than emittance-dominated. That is, the beam size in the focusing channel is essentially determined by the charge of a bunch of particles (which all have the same charge sign, positive in this case, and therefore repel each other) rather than by the beam's emittance, or the thermal motion of the individual particles.
} 
range. This permits considerable growth over the several-kilometer length of a driver. Consequently there has been a vigorous effort at several laboratories to understand the nature and control of this instability. While we and others search for means of direct experimental investigation, the instability has been studied with one- and two-dimensional particle-in-cell simulations, examinations of feedforward control, impedance-model calculations, and a variety of other theoretical approaches. We have also performed experiments with a scaled driver module (Figure 1-7).

The model duplicates the rf properties of an actual acceleration module from a driver. Data from these rf studies were used to validate our computer models of how the ion beam in a driver would react with the accelerating units at frequencies relevant to the longitudinal instabilities. ILSE will not be long enough for a complete test of these instabilities, although some driverlike beam-dynamics experiments will be possible.

The model core is nearly driverlike in size with an inner diameter of $1 \mathrm{~m}$ and an outer diameter of $2 \mathrm{~m}$. The area between would be filled with ferromagnetic material in a real core. In this model, about 1 out of every 10 $\mathrm{cm}$ along the radius is filled with Metglas $2605 \mathrm{CO}$ ferromagnetic tape; the rest is filled with aluminum ducting to maintain the approximate capacitance of a real core. (The capacitance plays an important role in the frequency response of the coupling impedance that describes the interaction between the beam and the core.) The 1-in-10 filling allows us to test the core at $20 \mathrm{kV}$ rather than $200 \mathrm{kV}$ and makes it convenient to probe voltages and currents within.

A February 1992 workshop on longitudinal instability took recent information from various laboratories into account. It seems that growth length of low-frequency disturbances ("low-frequency" in comparison to the



CBB 909-8127
Figure 1-7. In an early look at some characteristics of a driver, we are experimenting with this model of a driver-sized induction core. It is only $10 \%$ filled with ferromagnetic material, so we can experiment at about $10 \%$ of a driver's voltage. The aluminum ducting helps model the capacitance of a driver core, overcoming the effects of the partial filling. 
The Road Ahead

Figure 1-8. A conceptual drawing shows the scale and some postulated technical details of a power plant that uses heavy-ion induction linacs as drivers for inertial fusion energy. frequencies corresponding to typical 500-ns pulse widths) can be increased toward the hundreds-of-meters end of the range of possibilities, and that these disturbances can probably be countered with feedforward control systems, which would be necessary in any case. At high frequencies (tens of megahertz), the low quality factors at resonance of the induction cores should allow the expected momentum spreads to effectively damp resonant instabilities. Work continues on these longitudinal instabilities, but it seems likely that the unstable modes can be predicted and either avoided or controlled.

Figure 1-8 shows an artist's conception of an inertial-confinement power plant based on a heavy-ion driver. Obviously this installation will be much larger than the present-day experimental systems. Between ILSE and a driver (sidebar) at least one intermediate step will be needed, probably at an energy in the range of 10-100 kJ. This "Intermediate Driver Facility," or IDF, will allow us to understand two key areas of physics that cannot be addressed at a smaller scale.

One key physics area that would be explored with the IDF is costeffective avoidance or control of possible high-current instabilities; such research can be done with good confidence only in an experimental test at this scale. Another key area is the interaction of high-power ion beams with the high-temperature, solid-density matter of a target that is being compressed.

Meanwhile, research in other areas will have continued at various laboratories. The critical technologies of inertial-confinement fusion-driver, target, mass-production target factory, and reactor-are all equally necessary, like the four legs of a stool. There has been strong support in major

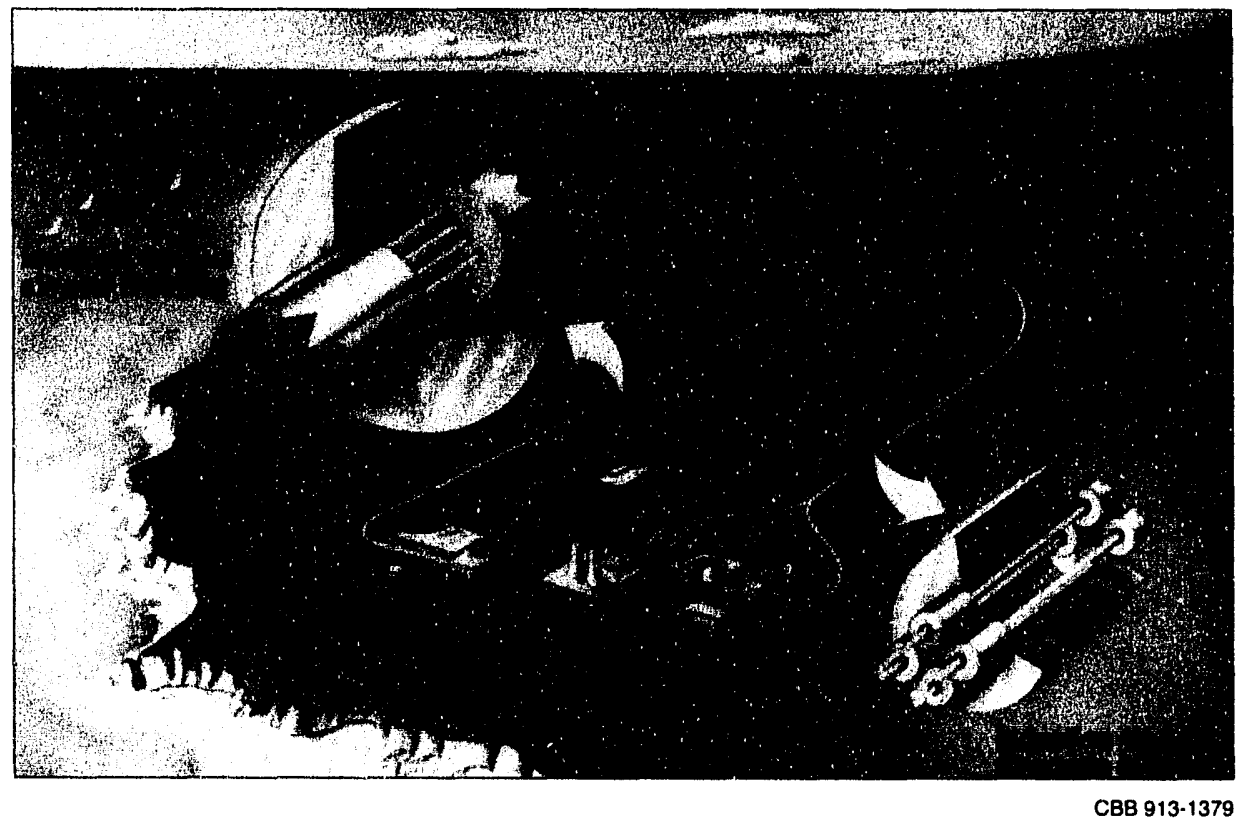

- This matter has few electrons per cubic Debye length and therefore cannot be called a "plasma," but is similar in some respects. 
review committees for a facility that would use 1- to 2-MJ lasers to explore target-implosion physics in the ignition regime with significant gain. Preliminary planning for such a facility is beginning at several DOE laboratories.

Reactor ideas have also been investigated at several laboratories and industrial firms worldwide. It would be appropriate to begin their further development in earnest as the target-implosion results become available.

The decision point for extending driver development to the megajoule level is at least 15 years in the future, and will depend on two main factors: results from the target experiments and the national need to move forward to an Engineering Test Reactor (ETR). An ETR would bring together the essential new pieces of an IFE power plant: the reaction chamber, the target design; the means for mass production of the targets; and heavy-ion driver experience from the IDF. It could support a full-scale test of all devices and processes involved in a reactor except conversion of fusion energy into electricity and recovery of tritium.

\section{Driver Candidates}

There are a variety of ways to "drive" a deuterium-tritium target, or impart sufficient energy to it (about $10^{15} \mathrm{~W}$ ) to cause fusion. LBL is investigating one of these approaches-the heavy-ion induction linac. Other laboratories are studying and experimenting with lasers and beams of lighter ions (to date, ranging from protons through lithium), and the German laboratory GSI is pursuing rf-accelerator options for heavy-ion drivers. The requirements for a driver are quite stringent; they include

- Cost, if IFE is to be an attractive power-plant option.

- Power sufficient to drive the target.

- Repetition rate (a few pulses per second; the lower limit is set by the maximum size of explosion the reaction chamber can handle in each shot, and the upper limit is set by the chamber's ability to prepare for the next shot).

- Shot-to-shot reliability comtined with long lifetime.

- And, because a power plant must have a very substantial net output, efficiency.

Lasers, such as Nova at LLNL, have been investigated for some time in the context of inertial-confinement fusion. Glass lasers like Nova can be made quite powerful, but at this time, pending further R\&D on cooling techniques, they have low repetition rates because of the time needed for the glass to cool between "shots." Also, today's lasers are only a few percent efficient, whereas heavy-ion drivers are projected to achieve operating efficiencies of $30 \%$ or more.

For an economical power plant, the product of driver efficiency and target gain will have to be greater than about 10 . Because target gain (the energy produced in the fusion reactions, divided by the energy put in by the driver) is expected to be on the order of 100 , driver efficiency is a stringent criterion. Lasers definitely have a place in inertial-confinement fusion and in defense research as the most-advanced drivers for target experiments, but their candidacy as power-plant drivers will remain uncertain until the efficiency and repetition-rate issues are resolved.

Light-ion diode accelerators are also being studied for this purpose, notably at Sandia National Laboratories. The disadvantage of light ions is that much greater beam current is needed to achieve sufficient power at the proper energy. The proper energy, in turn, is a function of the necessary range of target penetration. For heavy ions, the energy is higher (a few $\mathrm{GeV}$ versus tens of $\mathrm{MeV}$ ) but attainable. The beam current, a more troublesome parameter, is lower for heavy ions (kA versus MA), suggesting that collective effects might be much less severe. Both the Fusion Policy Advisory Committee and a National Academy of Sciences panel have recommended that the heavy-ion approach be developed further. 


\section{Publications and Presentations}

J.J. Barnard, A.L. Brooks, J.P. Clay, F.E. Coffield, E.J. Deadrick, L.V. Griffith, A.R. Harvey, D.L. Judd, H.C. Kirbie, V.K. Neil, M.A. Newton, A.C. Paul, L.L. Reginato, G.E. Russell, W.M. Sharp, H.D. Shay, J.H. Wilson, and S.S. Yu, "Study of recirculating induction accelerators as drivers for heavy ion fusion," Lawrence Livermore National Laboratory report UCRL-LR-108095 (1991).

S. Caspi, M. Helm, L.J. Laslett, W.M. Fawley, and M. Berz, "The use of numerical methods and differential algebra in magnetic field computation," abstract for the Spring Meeting of the American Physical Society (Washington, DC, 1992).

E. Close, C. Fong, and E. Lee, "The HILDA program," LBL-31917 (1991).

T. Fessenden and C. Fong, editors, Induction Linac Systems Experiments Conceptual Design Report, LBL document PUB-5324 (limited distribution, 1992).

S. Eylon and E. Henestroza, " $\mathrm{C}^{+}$source for the heavy ion fusion final focusing experiment," abstract for the 32nd Annual Meeting of the American Physical Society, Division of Plasma Physics (Seattle, WA, 1991); LBL-30994a (1991).

S. Eylon and E. Henestroza, " $\mathrm{K}^{+}$source for the heavy ion fusion final focusing experiment," 32nd Annual Meeting of the American Physical Society, Division of Plasma Physics (Seattle, WA, 1991); LBL32604a (1991).

S. Eylon, E. Henestroza, W. Chupp, and $\mathrm{H}$. Rutkowski, "Low emittance $\mathrm{K}^{+}$ion source for heavy ion fusion linear induction accelerators," 32nd Annual Meeting of the American Physical Society, Division of Plasma Physics (Seattle, WA, 1991); LBL32605a (1991).

W. Fawley, "Design of large aperture ratio magnetic quadrupoles for transport of heavyion fusion beams," Spring Meeting of the American Physical Society (Washington, DC, 1992); LBL-31699a (1992).

William M. Fawley, "ILSE: The next step in heavy ion fusion research," Journal of Fusion Energy 10, 297-298 (1991); LBL-31412 (1991).

T. Fessenden, R.O. Bangerter, D. Berners, J. Chew, S. Eylon, A. Faltens, F. Deadrick, and A. Friedman, "ILSE: the next step toward a heavy ion induction accelerator for inertial fusion energy," in Procedtings of the 14th International Conference on Plasma Physics and Controlled Nuclear Fusion Research (Würzburg, Germany, 1992); LBL-32213 (1992).

E. Henestroza, S. Eylon, W. Chupp, and $H$. Rutkowski, "High brightness $\mathrm{K}^{+}$ion source for heavy ion fusion linear induction accelerators," in Proceedings of the 1992 IEEE International Conference on Plasma Science (Tampa, Florida, 1992); LBL-32039a (1992).

William B. Herrmannsfeldt, Roger O. Bangerter, Rudolf Bock, William J. Hogan, and John D. Lindl, "Inertially confined fusion using heavy ion drivers," 15 th Congress of the World Energy Council (Madrid, Spain, 1992); Stanford Linear Accelerator Center report SLAC-PUB-5457 (1992).

Dennis W. Hewett, William L. Kruer, and Roger O. Bangerter, "Corona physics of heavy ion fusion targets," invited talk for the European Research Conference on Nuclear Physics: Prospects for Heavy Ion Fusion (Aghia Pelaghia, Crete, Greece, 1992).

HIFAR Staff, "Heavy Ion Fusion Accelerator Research (HIFAR) year-end report, April 11, 1991-September 30, 1991," LBL-31943 (1991).

William J. Hogan, Roger Bangerter, and Gerald L. Kulcinski, “Energy from inertial fusion," Physics Today 42, 9 (September 1992) pp. 42-50.

Hugh C. Kirbie, George J. Caporaso, Mark A. Newton, and Simon S. Yu, "Evolution of high-repetition-rate induction accelerators through advancements in switching," 16th International Linac Conference (Ottawa, Ontario, Canada, 1992).

E.P. Lee, "Beam dynamics and longitudinal instabilities in heavy-ion-fusion induction linacs," 16th International Linac Conference (Ottawa, Ontario, Canada, 1992); LBL-32813 (1992).

Edward P. Lee, "Heavy ion driven LMF design concept," Journal of Defense Research 21, 2 (May 1992); also in the Laboratory Microfusion Capability Study, Phase II report, to the U.S. Department of Energy (InterScience, Inc., 1993; LBL-31248 (1991).

E. Lee, "Longitudinal instability in accelerators for heavy ion driven inertial fusion energy," 32nd Annual Meeting of the American Physical Society, Division of 


\section{HEAVY-ION FUSION ACCELERATOR RESEARCH}

Plasma Physics (Seattle, WA, 1991); LBL32720A (1991).

Edward P. Lee, editor, Proceedings of the Longitudinal Instability Workshop,

(Berkeley, CA, 1992), LBL-32331 (1992).

Edward P. Lee, F.C. Younger, A.C. Paul, V.K. Neil, and E.E. Nolting, "Beam steering technology," Journal of Defense Research 21, 2 (July 1992), chapter contributed to Special Issue on Charged Particle Beam Weapons, edited by H.L. Buchanan, pp. 575-590.

E.P. Lee, H.D. Shay, A. Friedman, D.P. Grote, and J.J. Barnard, "WARP calculations of the proposed ILSE drift compression experiment," European Particle Accelerator Conference (Berlin, Germany, 1992).
R.A. Lindgren, B.L. Clausen, G.S. Blanpied, J. Hernandez, C.S. Mishra, W.K. Mize, C.S. Whisnant, B.G. Ritchie, C.L. Morris, S.J.

Sesstrom-Morris, C. Fred Moore, P.A. Seidl, B.H. Wildenthal, R. Gilman, and J.A. Carr, "Pion scattering to 6 - stretched states in ${ }^{24} \mathrm{Mg}$ and ${ }^{26} \mathrm{Mg}$," Phys. Rev. C 44, 6 (December 1991).

L. Smith and E. Lee, "Longitudinal instability of an induction linac with acceleration," accepted for the 1993 IEEE Particle Accelerator Conference; LBL-32902 (1992).

S.S. $\mathrm{Yu}$, "Induction accelerator approaches to heavy-ion fusion," Bull. Am. Phys. Soc. 37, 2 (April 1992), p. 2010 

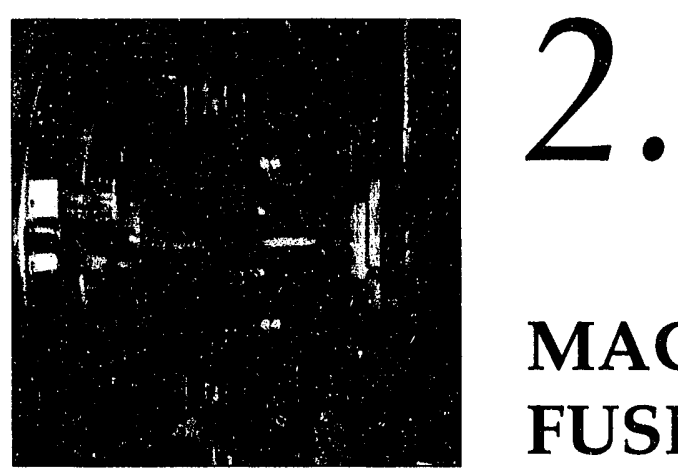

\title{
MAGNETIC FUSION ENERGY
}

\begin{abstract}
$\mathbf{T}$
HE PROPOSED INTERNATIONAL THERMONUCLEAR EXPERIMENTAL reactor, or ITER, is the next logical step in the worldwide magnetic-confinement fusion program. Its goals include "ignition" of the plasma and are likely to include self-sustaining "burn" for as long as two weeks at a time. Since 1988, the major portion of our Department of Energy-funded work in AFRD's Magnetic Fusion Energy Program has been directed toward this ambitious project. In 1992, we continued refining our design for a prototype neutral-beam injection system for ITER and our proposal to build a test facility capable of accommodating a 1.3-MV negative-ion system at currents of $1 \mathrm{~A}$ or better. We have also continued development of ion sources and accelerators.

Our expertise in these areas is not limited to fusion research; activities have been diversified considerably during the past few years. Ion sources and accelerators have industrial uses such as ion implantation for semiconductor processing and metal surface hardening. Our program also has an academic component centering on advanced plasma theory. Moreover, we have become involved in the development of an innovative permanent-magnet cyclotron, sometimes called a "cyclotrino," whose cost and size could make accelerator mass spectroscopy much more accessible as an analytical technique.
\end{abstract}

\begin{tabular}{|c|c|}
\hline w. Cooper ifregram had & $\begin{array}{l}\text { Plasma Applications } \\
\text { 1. Brown (Mromp lenter) }\end{array}$ \\
\hline Test Fucilities & A. Anders \\
\hline J. Kwan (group liater) & S. Anders \\
\hline C. Ackerman & B. Castro \\
\hline W. Steele & Z. Deng \\
\hline D. Williams & $\begin{array}{l}\text { M. Dickinson } \\
\text { Z. Feng }\end{array}$ \\
\hline Ion Source Technology & 1. Foijas \\
\hline K.-N. Leung (group latalder) & R. MacGill \\
\hline P. Hers & $x . \operatorname{las}$ \\
\hline \multicolumn{2}{|l|}{ C. I.i } \\
\hline L. Pan & Applied Theory \\
\hline L. Perkins & M. Vella (srouplesuler) \\
\hline D. P'once-Marquez & O. Andersonn" \\
\hline A. Young & C.F. Chan \\
\hline \multirow{2}{*}{$x . Y u$} & W. Kunkelt"* \\
\hline & A. Weist \\
\hline
\end{tabular}

Engineering Division, LBI

Department of I'hysics. University of California at Berkeley

Retired

" Ciraduate Student Research Assistant

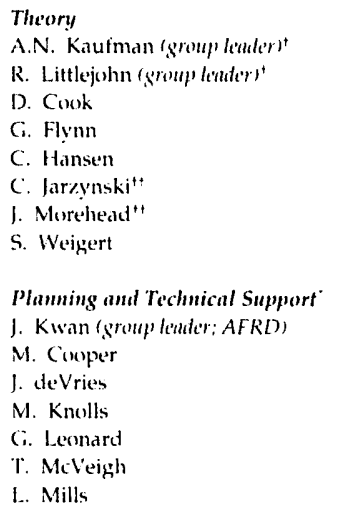

D. Moussa

M. Ramella

A. Rawlins

M. Rickard

T. Stevens

R. Wells

S. Wilde

Administrative Support

A. Aitkens

W. Scharf

B. Thibadeau

Students, Visitors, and Guests

A. Anders

S. Anders
D. Couk"

Z. Deng

Z. Feng

C. Flym"

P. Fojas

C. Hansen ${ }^{+t}$

i. Hers

C. Jarzynski"

1. Murchead ${ }^{+1}$

c. l.i

I. I'an

L. Perkins

D. Pence-Marquee

A. West

S. Weigurt

$x$. Yu 
Heating a plasma to thermonuclear temperatures is one of the many significant challenges in fusion-energy research, especially for the extended periods that will be needed as the next generation of tokamaks incorporate engineering tests and materials research as well as demonstration of ignition and burn. The primary focus of the MFE Group at LBL is development of neutral-beam injector systems for this purpose. The group's 20 years of work in this field began with the invention of novel multiampere positive-ion sources and of improved, computer-optimized acceleration systems. The most prominent achievement thus far has been the design, development, and transfer to industry of the Common Long-Pulse Source (CLPS). It is used in the Tokamak Fusion Test Reactor (TFTR) at Princeton and the DIII-D tokamak at General Atomics, two of the principal MFE experiments now operating in the U.S.

The CLPS has been highly successful, but it is based on positive-ion-beam technology, zolich is limited to a few hundred $\mathrm{keV}$ for efficient beam neutralization. In the next generation of tokamaks, such as ITER, larger plasmas will require higher injection energies - around 0.5 to $1 \mathrm{MeV}$, as compared to the 120-keV CLPS-for adequate penetration. Accordingly, we use negative ions, accelerating them to the necessary energies and subsequently neutralizing them by the simple process of detaching the extra electron. In contrast to systems based on positive ions, the neutral-particle yield of negative-ion systems does not decrease with increasing energy. However, it is difficult to produce large quantities of negative ions. Efforts to develop suitable sources of negative hydrogen ions at the ampere or multiampere levels are now underway here and at several other laboratories.

Design, construction, and testing of prototype accelerator systems must go hand in hand with development of a negative-ion source, so a substantial effort has been devoted to accelerator development.

After our design and proof-of-principle test of the ion source and accelerator, production of ITER's neutral-beain system would be handled by private industry.

In ITER, as defined by the Conceptual Design Activity, neutral deuterium beams with a total power of about $75 \mathrm{MW}$ will be injected to heat the plasma and to drive the toroidal current in the center of the plasma during steadystate operation, as shown in Figures 2-1 and 2-2 and explained in the sidebar. The energy needed to ensure the required plasma penetration, $1.3 \mathrm{MeV}$, is an order of magnitude greater than that of the CLPS. Beam steering is another necessary feature, as is steady-state operation for as long as two weeks. This combination of energy, current, and pulse length has never been achieved; further, the plasma generator and accelerator must be compatible.

To meet these needs, we are proposing a neutral-beam injection system based on negative-ion sources and our constant-current, variable-voltage (CCVV) electrostatic accelerator design. The design was refined during 1992 after extensive interaction with our fellow participants in the ITER Conceptual Design Activity. * After pre-acceleration of the beam to $300 \mathrm{kV}$, a short matching stage focuses the beam and feeds it into the first acceleration stage. Each acceleration stage increases the beam energy by as much as $125 \mathrm{keV}$. The acceleration stages use electrostatic quadrupoles for focusing.

\footnotetext{
- The other three ITER partners (the European Communities, Russia, and Japan) are examining variations of a different electrostatic accelerator technology that uses electrostatic weak focusing for space-charge control and magnetic fields for secondary-particle control. Because of the risk and the tight deadline, the ITER Conceptual Design Activity R\&D plan calls for concurrent development of both approaches.
}

\section{Neutral-Beam Injection for ITER}

\author{
Accelerator Proof-Of- \\ Principle Experiment
}


Figure 2-1. The proposed International Thermonuclear Experimental Reactor is an ambitious scientific and technological step toward a demonstration power reactor. LBL's role within the U.S. effort involves the design and development of neutral-beam systems to heat the plasma and drive the toroidal current. The artists' renderings show approximately how ITER's "core" (right) compares in size to that of the Tokamak Fusion Test Reactor now running at the Princeton Plasma Physics Laboratory (above). (After PPPL and LLNL artwork.)

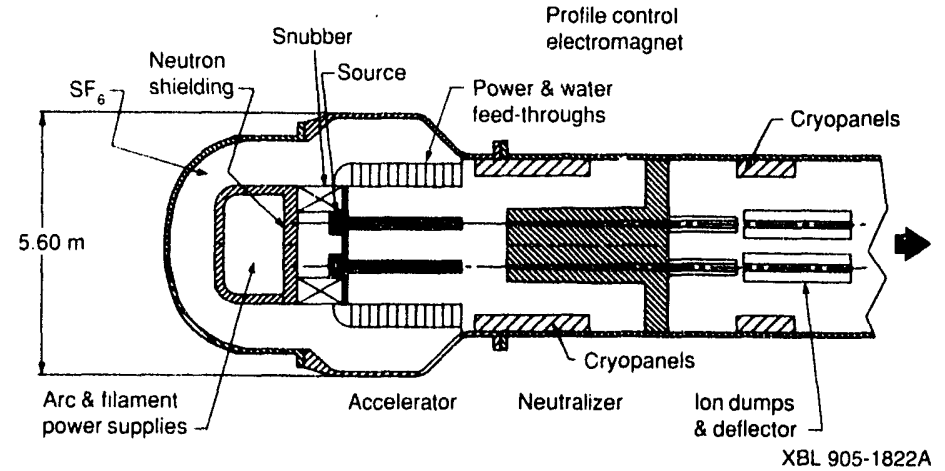

Figure 2-2. The ITER conceptual design calls for three stacks of three 1.3-MeV neutral-beam injector modules providing a total of $90 \mathrm{MW}$. Each injector can provide $10 \mathrm{MW}$, so ITER can continue to operate if one of them is down for repair or modification. The performance specifications are ambitious, especially in terms of pulse length-two weeks, as opposed to a few tens of seconds for today's NBI systems. (Overall layout diagram courtesy of LBL and Grumman.) 
For ITER neutral-beam-injection applications, the energy range of the CCVV accelerator has to be widely variable. The output beam energy may be varied by tuning the acceleration voltages and the ESQ focusing voltages; this may be performed rapidly without altering the accelerator's mechanical configuration. Because the electrostatic quadrupoles provide strong focusing (as contrasted with designs that depend on the accelerating electrodes for weak focusing), the energy can be varied without requiring a change in the current. The average accelerating gradient can be kept as low as $3-5 \mathrm{kV} / \mathrm{cm}$ to enhance insulator lifetime. Another advantage is that the transverse electric fields sweep away electrons at a mean energy of $64 \mathrm{kV}$ (in the ITER design), minimizing $x$-ray generation. Secondary positive ions are similarly swept away. Figure $2-3$ shows the proposed accelerator in schematic form.

In 1992 we completed a computational design for a $1.3-\mathrm{MeV}, 1.4 \mathrm{~A} \mathrm{H}^{-}$ ESQ accelerator and preaccelerator. The design offers good operability at the design emittance of $2.0 \pi \mathrm{cm}$ milliradians and, at an emittance of 1.0 $\pi \mathrm{cm}$-mrad or less, is capable of currents as high as $2 \mathrm{~A}$. We also made the system more compact by finding a way to integrate the power supplies for the ESQ electrodes into the highvoltage shielding rings alongside the accelerator. The facility for the proposed LBL Proof-Of-Principle Experiment (POPX) for the ITER accelerator design is shown in Figure 2-4.

If approved, the POPX hardware will be built at an existing site, suitably modified, within the Bevatron accelerator complex. This is already a controlled-access radiation area, and features such as enclosed floor space, a large overhead crane, adequate utilities, and concrete shielding blocks are available. In May 1992 the conceptual design was reviewed favorably. The project has an estimated cost of $\$ 14.7$ million and will take approximately 30 months. DOE funding is on hold pending the ITER Council's decision on whether to use neutral beams in the first phase of ITER operations. the highly conductive plasma.

\section{Neutral Beams and Current Drive}

In addition to their primary role of heating the plasma, injected neutral beams help confine and control it. In a tokamak, the plasma is confined by the combination of two principal magnetic fields: a toroidal field in the plane of the torus and a poloidal field wrapped around it, as shown here.

A fusion plasma is very hot-that is, the charged particles move high speeds. In the absence of a magnetic field, they would move the torus, spiraling along the lines of force. This field is generated by external coils. Under the influence of the toroidal field alone, the a would move toward the outer wall, so a poloidal field is added. The poloidal field is the result of a very large electrical current-about twenty million amperes in ITER-coursing through

This current in the plasma is initiated inductively by a The resultant "ohmic heating" is not sufficient to reach hermonuclear temperatures and must be supplemented, but will be cient for driving the plasma current during the initial physics hase of ITER operation.

However, as the ITER Council defines the reactor's mission, they may retain the plans for a subsequent technology phase that will vive sustained burns of up to two weeks. This is far beyond a monotonically increasing voltages that are needed, so supplemental ninductive drive will be required

In these longer experiments, the bulk of the current could be plasma. (The beams are neutralized so that they can pass through the magnetic fields; after entering the tokamak, they are re-ionized Coulomb interaction.) 
Figure 2-3. The accelerator that would be used for the POPX is a CCVV accelerator with ESQ focusing, shown here in schematic form. It is a scaled-up version of ("ne we have been working with since 1987 (photo), with quadrupole units approximately 2.5 times larger in length and diameter.

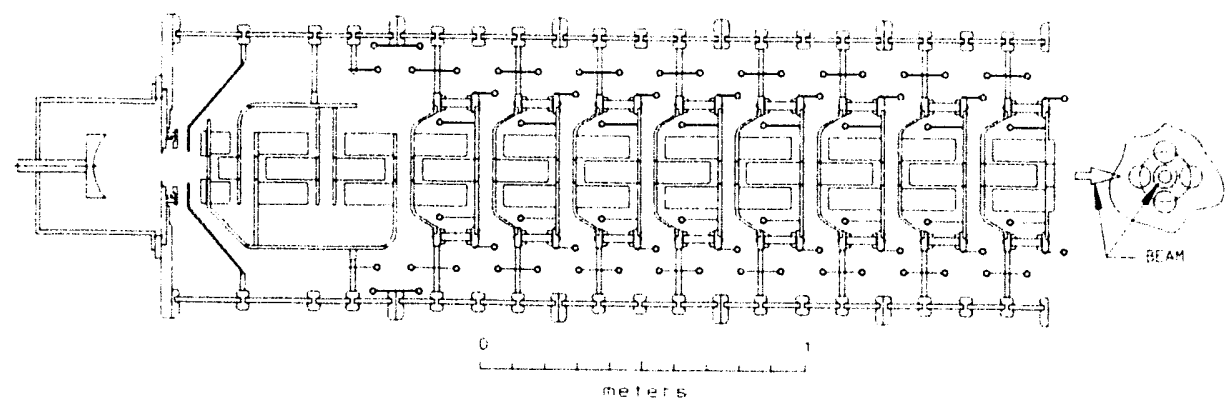

XBL $917-6770$

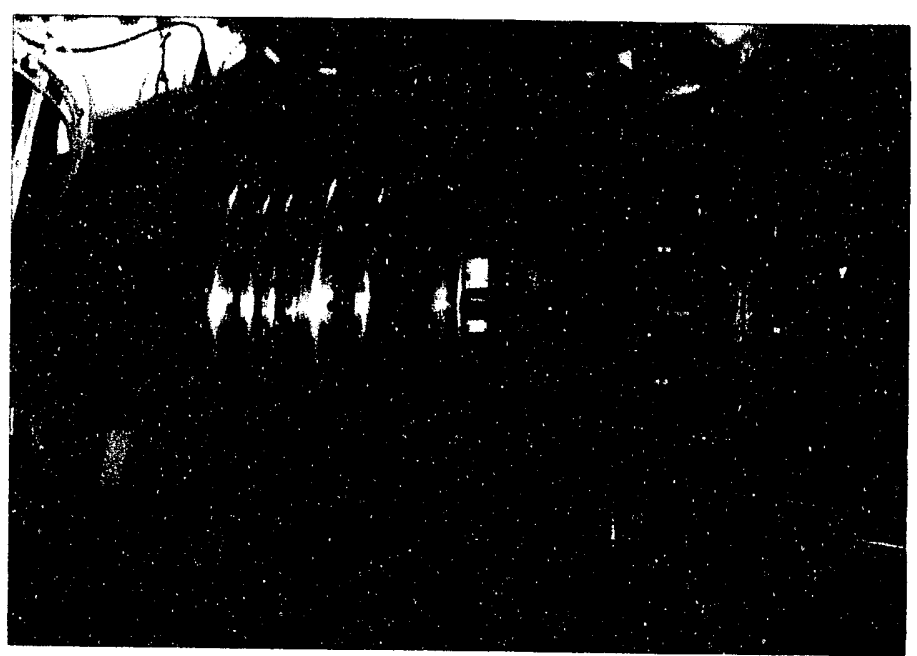

XBC 880-10009
The goal of the POPX is to accelerate 1.4 A of $\mathrm{H}^{-}$to $1.3 \mathrm{MeV}$ for two seconds. This test will represent a single channel of the accelerator portion of the actual multichannel accelerator module for ITER neutral-beam injection. Each of the 16-channel ITER beam modules will also include a beam neutralizer and ion-beam dumps. Each module must provide a 1.3-MeV $\mathrm{D}^{0}$ beam that would have a current of $7.7 \mathrm{~A}$ if charged.

Transfer of this technology to U.S. industry remains an important goal of our program, which in the past has contributed to positiveion-based systems produced by McDonnell-Douglas for TFTR and by TRW for Doublet III, as well as the Common Long-Pulse Source built by RCA for TFTR and Doublet III-D. Negative-ion-based neutral-beam systems operating at $500 \mathrm{keV}$ are part of the proposed TFTR-M upgrade and might be incorporated in the proposed Tokamak Physics Experiments, or TPX, at Princeton. Positive-ion technologies in the 120-150 keV range could also return to our program in the form of proposed beam-plasma neutron sources that would be used for development of fusion materials.

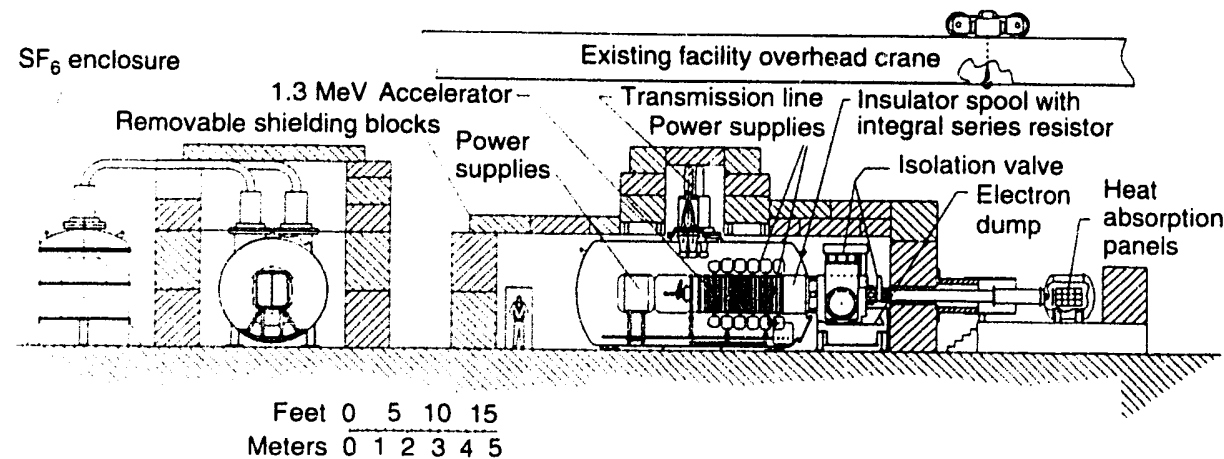

XBL $9212-5856$
Figure 2-4. The goal of the POPX is to accelerate $1.4 \mathrm{~A}$ of $\mathrm{H}^{-}$to 1.3 $\mathrm{MeV}$ for $2 \mathrm{~s}$ in this proposed facility, which could be built in the Bevalac complex. This test will represent a single channel of the accelerator portion of the actual multi-channel accelerator module for ITER neutral-beam injection. Each ITER neutralbeam module will also include a beam neutralizer. It must provide a $1.3-\mathrm{MeV} \mathrm{D}^{0}$ beam that would have a current of $7.7 \mathrm{~A}$ if charged. 
It is not yet clear which of several negative-ion-source technologies will be best suited to the high-current, long-pulse needs of future neutral-beam injection systems. One

\section{Ion Sources}

of our earliest efforts, a "surface-conversion" source in which hydrogen ions were" produced on the surface of a cesium-coated molybdenum electrode in a hydrogen plasma, achieved the first steady-state yield of more than an ampere of $\mathrm{H}^{-}$. However, the partial cesimm coating-required in order to optimize the ion yield-risked the undesirable side effect of potentially contaminating the accelerator dozunstream.

Much of our work has continued to focus on surface-conversion sources using less-volatile coating materials such as barium. A second component of our ionsource program focuses on "volume-production" sources (sidebar) that produce ions throughout a volume of gas rather than on the surface of an electrode. (We have also begun a promising collaboration with the Japan Atomic Energy Research Institute that may lead to better ion sources for ITER and other applications.) The main goal is to increase the steady-state current capability of these sources. In the meantime, we have continued to obtain promising results with an rf-driven surfaceconversion scheme. Already being used in spinoff applications, including the Superconducting Super Collider, rf generation of the plasma could eventually supplant conventional schemes for very-long-pulse. operation. And, in the latest stage of an ongoing effort, we are developing a negative ion source for the U.S. Army Strategic Defense Command's neutral particle beam program.

We are also investigating photocathodes, bright electron sources that offer a singular feature: electron pulses of the same length as the laser pulses that excited the cathode material. We are attempting to make them both efficient and rugged enough for practical applications. Work also continues in the laser and spectroscopic diagnostic of plasmas in our group's various ion sources.

\section{Production of Negative Ions}

Volume production and surface conversion are two fundamentally different ways of producing negative ions. Both types begin with a gas of the desired species (hydrogen or deuterium in our work) that is partially ionized by any of several means, but thereafter the two methods diverge at the level of basic chemical physics.

In surface conversion, a negatively biased element (either a coated converter/cathode or a separate, coated converter element) draws positively charged ions from the plasma. Some of them are backscattered, a process in which they sometimes become transformed into negative ions by capturing two electrons from the metal surface.

Meanwhile, the surface has been adsorbing the species that makes up the plasma. Of the incoming positive ions that are captured rather than backscattered, some sputter the adsorbed atoms out of the surface; the atoms that are sputtered out can also emerge as negative ions.

The sheath of positive ions that surrounds the converter-a sheath a few tenths of a millimeter thick in an intense dischargeaccelerates the negative ions. Those that leave the source by this means are said to have been "self-extracted."

In volume-production sources, gas-phase reactions are dominant (though surface conversion can take place at the chamber walls) and vibrational excitation of diatomic hydrogen atoms is thought to play a key role. The model involves a two-step reaction:"

$$
\begin{aligned}
& \mathrm{H}_{2}\left(v^{\prime \prime}=0\right)+\mathrm{e}^{-}(\geq 25 \mathrm{eV}) \rightarrow \mathrm{H}_{2}\left(v^{\prime \prime} \geq 6\right)+\mathrm{e}^{-} \\
& \mathrm{H}_{2}\left(v^{\prime \prime} \geq 6\right)+\mathrm{e}^{-}(\approx 1 \mathrm{eV}) \rightarrow \mathrm{H}^{0}\left(v^{\prime \prime} \geq 6\right)+\mathrm{H}^{-}
\end{aligned}
$$

Reaction (2) can also work in reverse, and is thought to be an important mechanism for $\mathrm{H}^{-}$loss in the discharge. Using selective absorption of tunable laser radiation in the vacuum-ultraviolet region of the spectrum, we measure the densities of various groundstate and excited neutral components of the plasma. These measurements indicate that this simplified model, although incomplete, is essentially correct.

\footnotetext{
"Here $\mathrm{v}^{\prime}$ indicates a vibrational quantum level. The atoms, ions, and molecules undergo translational motion through space. The molecules also rotate, or tumble, about various axes, and, on a much faster time scale, they vibrate, which may be interpreted as expansion and contraction of the bonds between atoms. Translation occurs in the classical continuum, whereas the rotational and vibrational states are quantized (though described well by semiclassical treatments).
} 


\section{Progress with Surface- Conversion Sources}

\section{RF-Driven $\mathrm{H}^{-}$Source}

Figure 2-5. This surfaceconversion source with barium converter electrode appears likely to meet our output goal of $200 \mathrm{~mA}$ of $\mathrm{D}^{-}$; a similar source of about twice the size should provide $1 \mathrm{~A}$ of $\mathrm{D}^{-}$in a single beam, thus meeting the requirements for ITER neutral-beam systems.
Scaling up from earlier achievements in our ongoing development of surface-conversion sources, we have begun testing a new source with a $10-\mathrm{cm}-$ diameter barium converter. The source (Figure 2-5) is designed for steadystate production of $200 \mathrm{~mA}^{-}$of $\mathrm{D}^{-}$, representing a half-scale test of an ion source for ITER. It has an annular plasma generator, which, as indicated by earlier research, optimizes the uniformity and quality of the plasma delivered to the converter. Thus far, with a gas pressure of $1.5 \mathrm{~m}$ Torr and low converter current $(3.5 \mathrm{~A})$, we have measured a total of $150 \mathrm{~mA}$ with a calorimeter. Of that total output, $42 \mathrm{~mA}$ survived stripping and made it to the Faraday cup. The Faraday-cup intensity profile suggested that the transverse energy is $5-10 \mathrm{eV}$, which is within the requirements for ITER neutralbearn injection. We project that, with $12 \mathrm{~A}$ of converter current and $1 \mathrm{mTorr}$ of gas pressure, we will meet the $200 \mathrm{~mA}$ requirement, and we may exceed that figure if, as has been predicted, production efficiency increases with plasma density.

High-frequency rf (around $1.7 \mathrm{MHz}$ in our present work) offers a different and potentially more robust approach to generating the plasma in both volume-production and surface-conversion sources. Our rf-driven source is based on the same "bucket" with a multicusp magnetic field as the thermionic-cathode ion sources that we have been working with for several years. However, it has a porcelain-coated antenna instead of a filament cathode. The antenna is immersed in the plasma instead of external to the discharge chamber as in some other designs. The antenna's long-term survivability in the plasma has been demonstrated; the porcelain-coated antenna can maintain a clean plasma in continuous operation for a week or more. This is an attractive feature in high-power, steady-state applications. The rf energy sets up an oscillating magnetic field, which, in turn, induces an electric field that provides energy to the electrons, producing a plasma.

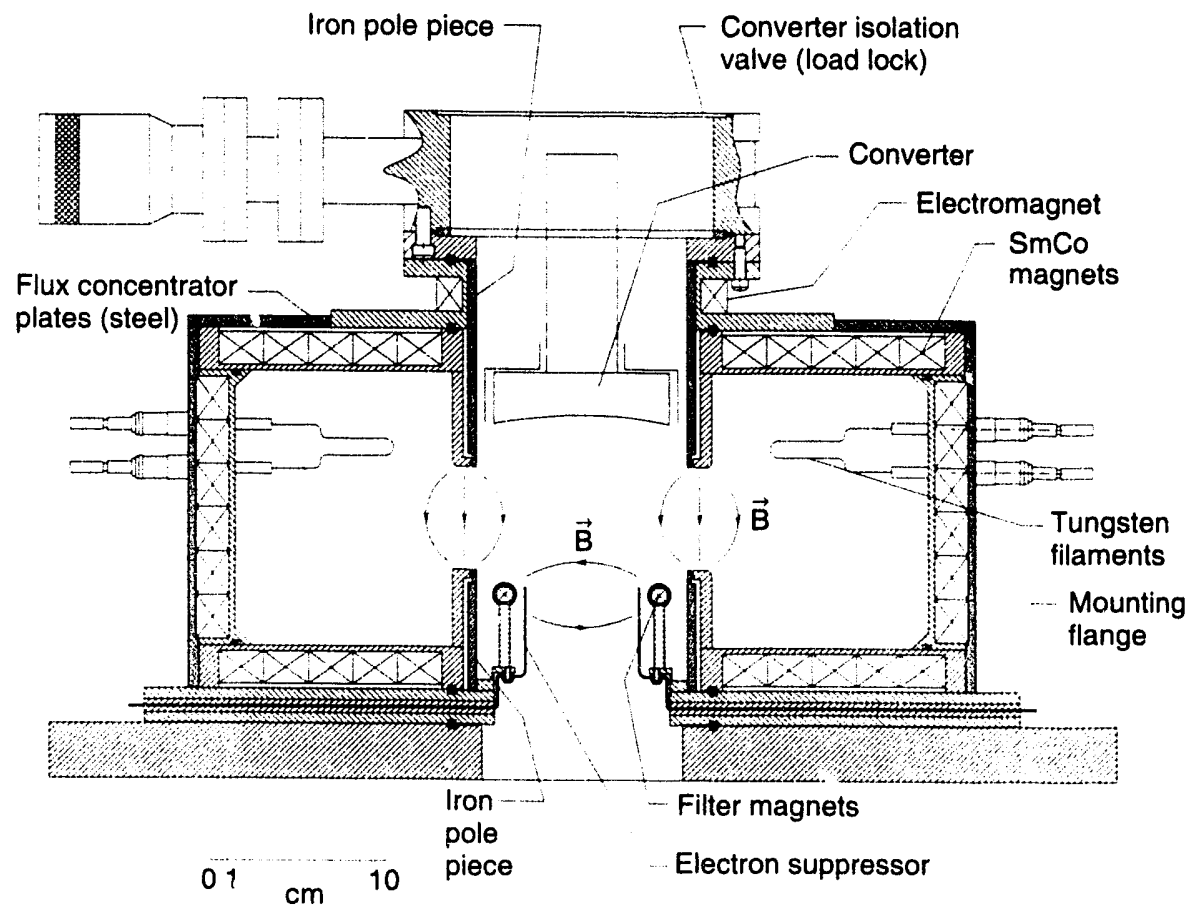

XBL $919 \cdot 2009$ 
As with the other negative-ion sources, addition of alkali metals such as cesium can greatly enhance the output current for applications where cesium contamination (which can cause voltage breakdown in downstream accelerator compon(ents) is not a major issue.

Since our work with it began in 1989, we have made continuing improvements in our $\mathrm{rf}$-driven $\mathrm{H}^{-}$source. A unit has been developed, under contract to AccSys Techrologies, for calihration of detectors in high-energy physics. Another unit is now being evaluated in the beamline test facility for the Superconducting Super Collider's injection system. Recent improvements to the basic design include optimization of the filter and collar geometries to raise the output of $\mathrm{H}^{-}$and lower the output of electrons, which are undesired. As the SSC boosters are commissioned, this ion source (Figure 26) will be retained, either as a backup for a more-conventional $\mathrm{H}^{-}$source or possibly as the primary injection source.

We have also designed an electrostatic injector to match the beam into the nex: : age of the SSC injection system, a radio-frequency quadrupole (RFQ) accelerator. The injector consists of four electrodes operating in an acceleration-deceleration-acceleration scheme. The first two employ an acceleration voltage u: $50 \mathrm{kV}$ to extract both the $\mathrm{H}^{-}$ions and the electrons from the source. The electrons are swept away by a filter consisting of a pair of permanent magnets. The extracted beam is decelerated by the third electrode, expanding as it slows, and is reaccelerated and compressed by the fourth electrode. This simple, compact accelerator and transport system can deliver $30 \mathrm{~mA}$, matched into the RFQ.

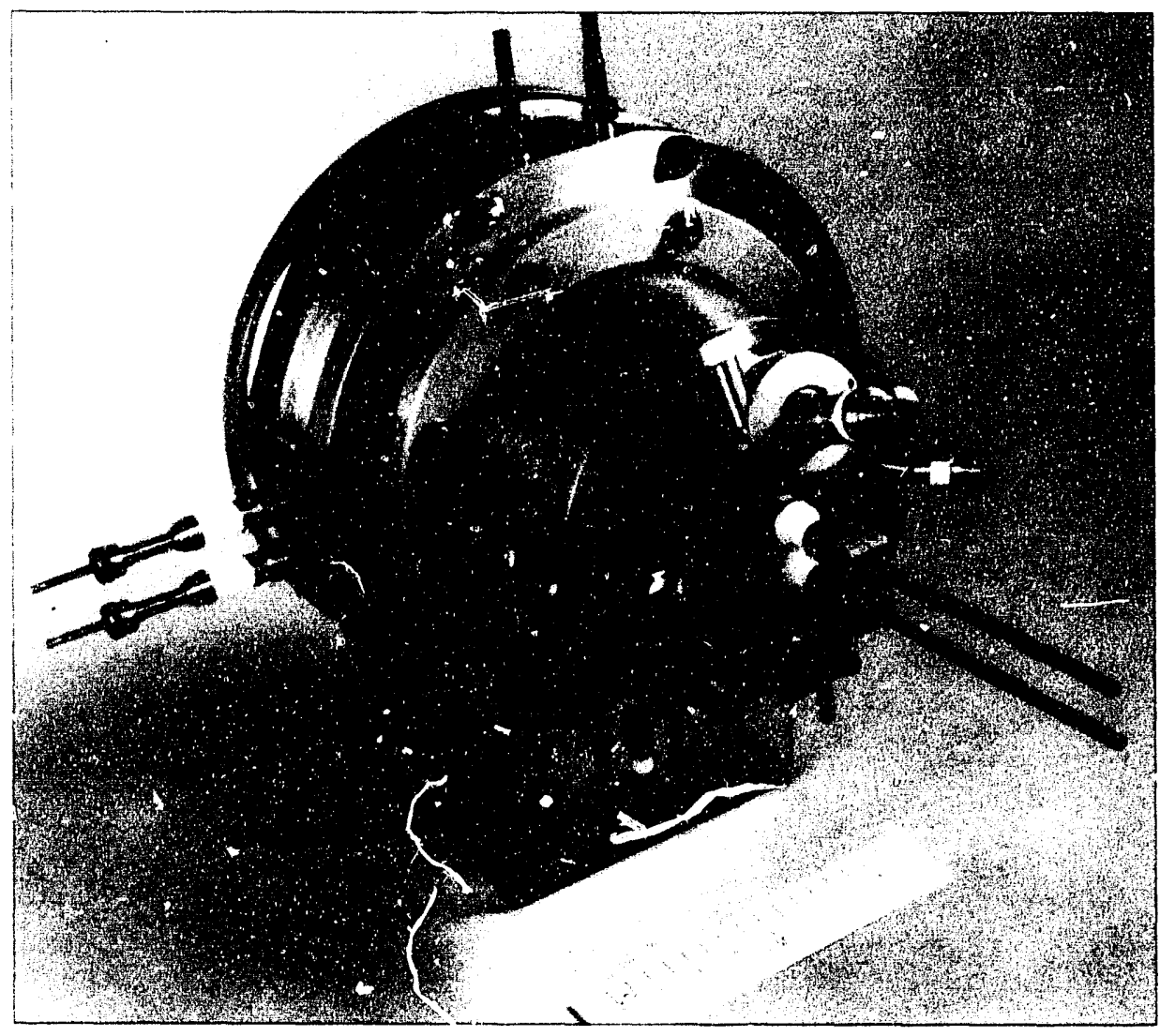

CBB 910-8; 14
Figure 2-6. This rf-driven $\mathrm{H}^{-}$ source, bu:lt by $L B L$, is now installed for Seamline test at the Superconducting Super Collider. It is a candidate for use as the primary SSC injection source. 
Cyclotron Miass Spectrometer

\section{Materials Modification and Synthesis}

Our rf-driven sources have a variety of applications outside the fusion and high-energy physics communities. One particularly promising application is ion implantation for hardening and otherwise modifying the surfaces of materials. The rf-driven sources can be readily configured to provide positive ions, and their characteristics make them attractive for industrial use. For example, a nearly pure beam of atomic nitrogen $\left(\mathrm{N}^{+}\right)$can be provided for hardening of metals. Other sources currently in use also put out $\mathrm{N}_{2}{ }^{+}$, which must be removed by a large and costly mass-separation apparatus because it forms an undesirable shallow implant layer. In the same vein, we have produced a source that provides a record concentration of $\mathrm{B}^{+}$ (greater than $40 \%$ ) from $\mathrm{BF}_{3}$ gas. This source will soon be installed and tested in an ion implantation machine by Varian Associates, a maker of semiconductor fabrication equipment, in the hopes of achieving a variety of improvements, including not only beam purity but also longer source life in the $\mathrm{BF}_{3}$ environment.

In collaboration with LBL's Physics and Engineering Divisions, we are continuing development of a compact axial-injection research cyclotron, sometimes known as a "cyclotrino," based on permanent magnets rather than the usual electromagnets. The new instrument will be used for ultrasensitive accelerator mass spectrometry, replacing the much larger and more expensive van de Graaff generators usually employed for this purpose. With its combination of sensitivity and small size-it will be portable, though in the sense of being carried by truck rather than by hand-the system will have the potential for great practical benefit. For example, exhausts and effluents could be checked for minute quantities of hazardous materials. Moreover, the instrument's predicted sensitivity will allow detection of tiny tracer concentrations of ${ }^{14} \mathrm{C}$, opening the door to many potential applications in environmental science, biomedical research, and archeology. To facilitate ${ }^{14} \mathrm{C}$ tracer work-an especially interesting and important application--the effort also encompasses optimization of a $\mathrm{C}^{-}$ source that uses gaseous $\mathrm{CO}$ or $\mathrm{CO}_{2}$ rather than sputtering of solid graphite.

The design of the cyclotron has been completed; construction is underway, and the instrument should be completed in 1993.

The performance, durability, and economic attractiveness of today's high-technology products are often predicated upon specialized materials and upon effective, affordable techniques for manufacturing them. A group within the MFE Program,i close interdisciplinary collaboration with colleagues from $L B L$ and elsewhere, inve'stigates plasma and ion-beam techniques for modifying and synthesizing materials. The program has four parts: development and use of metal-vapor vucuum arc ion sources for ion implantation; research on techniques for depositing metallic thin films and multilayers using metal-plasma guns; modification of surfaces through metal-plasma immersion (MPI) techniques; and attempts to deposit industrially useful diamond coatings on surfaces. Most of our investigations are deeply collaborative. A broadbased community of researchers in materials science and allied fields comes from other LBL divisions, other laboratories and universities, and private industry to take advantage of our expertise and facilities. 
In 1992, we continued the development of ion sources based on the metal-vapor vacuum arc principle, along with their use for high-dose metal-ion implantation. We conduct a wide range of ion-implantation experiments to demonstrate applications of the technology. Some examples of recent studies include implantation of $\mathrm{Ti}$ into $\mathrm{Pb}$ to improve the longevity of the lead plates in leadacid batteries; implantation of $\mathrm{Ti}, \mathrm{Cr}$, and ${ }^{-1} \mathrm{Ni}$ ions to improve the spark-erosion resistance and wear resistance of compon nts for electromagnetic rail guns; and reduction of friction in $\mathrm{SiN}$ ceramics (which are of interest for a variety of applications, including auto engines) by implantation of very high doses of $\mathrm{Ni}$. More-basic research projects aimed at a better understanding of ion implantation also play a role in our work. A 1992 highlight was a study of anomalously deep penetration at high dose and high temperature; by using doses of $\mathrm{Ti}$ as high as $1 ¥ 10^{18}$ ions $/ \mathrm{cm}^{2}$ at surface temperatures of approximately $600^{\circ} \mathrm{C}$, we implanted ions to depths of several thousand angstrorns in silicon.

We have made what might prove to be a breakthrough in practical ion implantation: development of a direct-current (continuous-beam) metal ion source that puts out a very wide beam. This requires not only a dc version of the plasma arc itself, but also an extraction area of unusually large cross section. We have demonstrated the feasibility of this apparatus, the DC Broad-Beam High-Current Metal Ion Source (Figure 2-7). In early tests, it delivered dc metal-ion beams of order $1 \mathrm{~A}$ across an area of a few tens $\mathrm{of}^{2}$ at an electrode voltage of $10 \mathrm{kV}$. (By contrast, other ion sources now commercially available provide beams of a few $\mathrm{cm}^{2}$ that have a current of 20-30 $\mathrm{mA}$. They use different technologies developed for applications like semiconductor processing whose beam requirements are much different.) It should be straightforward to scale up all parameters-including the most

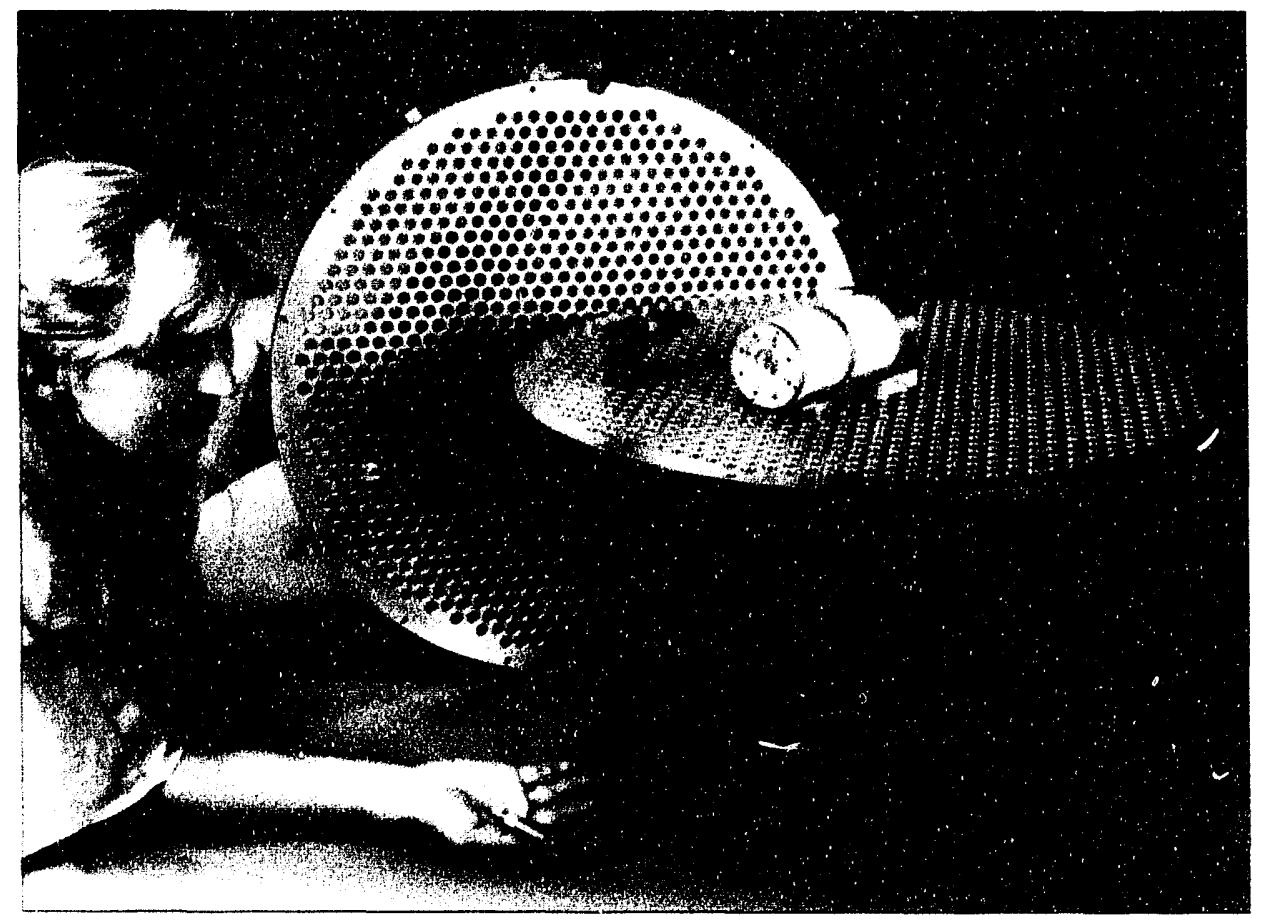

CBB $926-5122$
Vacuum Arc Ion

Source Development and Applications

Figure 2-7. As part of an array of proof-of-concept tests, our DC Broad-Beam High-Current Metal Ion Source was used to produce a 10-A, 100-keV beam of $\mathrm{Ti}$ ions. (Due to the limitations of our power supply, the $100-\mathrm{keV}$ test was conducted in pulsed mode.) Scaled up in a straightforward manner and incorporated into a complete materials-processing system, this technology could make some types of industrial ion implantation several orders of magnitude faster, accomplishing in minutes what now takes a day. Here the 50-cm-diameter electrodes are shown with previous generations of our metalvapor ion source technology, including a subminiature embodiment. 


\section{Metallic Thin Films and Multilayers}

\section{Metal Plasma Immersion (MPI) Surface Modification}

Figure 2-8. This Auger spectrum shows the thick, atomically mixed transition zone between an alumina film deposited with our Metal Plasma Immersion technique and the steel substrate upon which it was deposited. This "ion-stitched" interface provides very good adhesion between the ceramic film and the metal substrate. important one, ion current-producing an industrial ion source that is better than today's commercial technology by several orders of magnitude.

This giant bea!n provides the first opportunity for carrying out metallurgical ion implantation on a very large scale, with unit costs vastly reduced below the present. For example, with our prototype unit, a surface area of 1 square meter could be implanted with a dose of $1 ¥ 10^{17}$ ions $/ \mathrm{cm}^{2}$ in just a few minutes. With a conventional implanter this would take about a day. The new ion source (like the original metal-vapor vacuum arc ion source) has won an R\&D 100 award, the recognition that is given annually by Research and Development Magazine to the year's 100 most-promising new technologies.

Since 1991 we have been exploring applications of a technique we had developed for synthesizing and modifying materials with metal plasma guns. By adding gaseous species to the plasma, we can deposit not only metals, but also ceramics, oxides, and so forth. This results in a potentially powerful and versatile technique for fabricating metallic superlattices, multilayers, and thin films. We are investigating a number of possible applications, including catalytic surfaces, magneto-optical recording media, multilayer x-ray mirrors, diamondlike thin films of polycrystalline carbon, and films of new harder-than-diamond materials. The technique is also useful as a tool for fundamental research in surface science.

In this technique, which also takes advantage of our plasma-gun technology, the kinetic energy of a plume of metal plasma is controlled by adjusting a negative bias pulse applied to the target. Thus the incident plasma can be deposited on the target surface as a film or implanted energetically below the surface. In a program sponsored by the Electric Power Research Institute, we are learning to control the effects by adjusting the parameters of the incoming plasma plumes, the voltage of the bias pulses, and the phase relationship between the two. Among the features of this technique are the ability to create atomically mixed interfaces and to "stitch" a deposited layer into the substrate of another layer for greater durability (Figure 2-8).

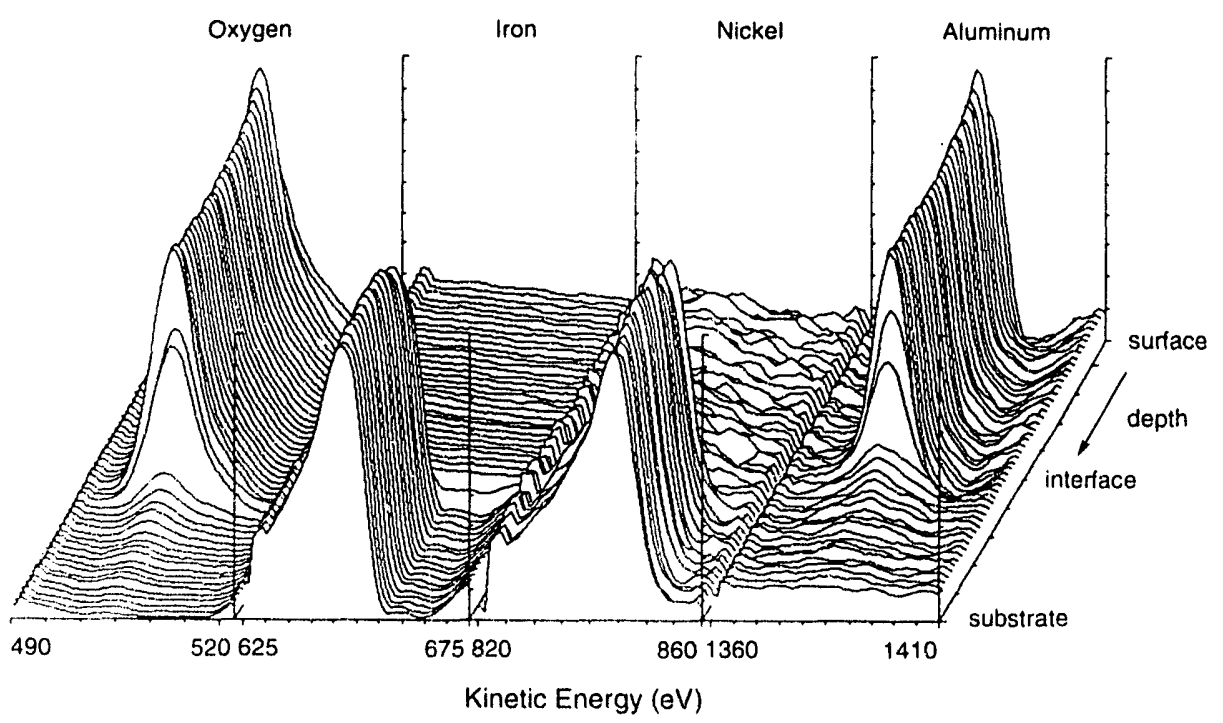

$X B L .928 \cdot 1832$ 
Together with the Mechanical Engineering Department of the University of California at Berkeley and with LBL's Materials Sciences Division, we are investigating the synthesis of polycrystalline diamond thin films on substrates that are of technological value. A suitable substrate is immersed in a microwave-produced hydrogen/methane plasma, and diamond films grow from the plasma state by chemical vapor deposition. The goal is to develop industrially applicable techniques for depositing diamond thin films onto large, three-dimensional substrates. We are now able to routinely synthesize high-quality films of polycrystalline diamond that are one to several $\mu \mathrm{m}$ thick and a few square centimeters in area. We are studying ways to bond the film to the substrate more strongly - an important requirement for moving such diamond films out of the laboratory and into applications. One such application, which we are pursuing, is the use of ultrathin diamondfilm coatings for protecting the magnetic "platters" used in computer hard disks.

The MFE Program at LBL maintains a plasma-theory group operating in the borderland zohere physics blends into mathematics. The group's pure and applied studies help other researchers understand the phenomena observed in hot plasmas and the possibilities for future development. We have sought new ways of analyzing gyroresonant absorption; the goal is to understand the plysics of the phenomenon and thereby describe it in simpler mathematical terms. Our work in this area has yielded not only simplified mathematical approaches, but also insights into the geometry of wave propagation in a plasma.

The immediate purpose of this work is to understand heating and wave transport in plasmas-in particular, gyroresonant absorption of energy. Ion cyclotron range of frequency (ICRF) heating, one of the important heating schemes for tokamaks, involves irradiation of the plasma by a coherent magnetosonic wave excited by an antenna. This radiation is partially absorbed at a resonance layer, where the wave frequency $\omega$ matches either twice the local gyrofrequency of a dominant ion species or the fundamental gyrofrequency of a minority species. In studying gyroresonant absorption, it is important to understand mode conversion (how and where the waves couple into one another) inside a tokamak.

In 1992 we extended our analytic treatment of ion-gyroresonant heating in tokamaks from the perpendicular-fluid model (appropriate to passing particles) to a kinetic model that accounts for trapped particles. The longterm goal, here and generally, is to advance from simple, analytically tractable problems to more-comprehensive, more-detailed treatments of realistic geometries and phenomena.

For many years, the theory of wave propagation in nondissipative media has been based on two implicit assumptions. First, the governing wavefunction is assumed to be Hermitian (non-Hermiticity being associated with dissipation). Second, the fundamental conservation law for local wave action is assumed to be based on eikonality (that is, the variation in amplitude is slow compared to the variation in phase). We have shown that both of these assumptions are false, introducing the concept of "pseudoHermiticity" (with respect to a Hilbert space of indefinite metric) and illustrating this with numerous practical examples.
Diamond Synthesis

\section{Plasma Theory and Nonlinear Dynamics}

\author{
Wave Dynamics and \\ Gyroresonant Energy \\ Absorption
}


This concept leads to a variational principle for which the wave field's local phase variation yields an action-conservation law without invoking eikonality. We have recently applied this law to several problems of mode conversion and have realized that the results may have useful implications in understanding other fields. Mode conversion is a wide-ranging scientific motif that, to take a few examples, occurs in seismology, stellar dynamics (where acoustic waves are coupled into gravity waves), and the reactive scattering observed in atomic and molecular physics. However, the practitioners of these various fields use different terminology for their different applications of mode conversion, and they travel in different circles. An effort to cross-cut these fields, looking at the similarities in theme rather than the differences in context, could reduce duplication of effort and provide analytical tools from which they all could benefit.

It is also known that, when a propagating wave converts locally to a damped non-propagating oscillation, the governing phase difference is sensitive to weak nonlinearity. We have shown that, because of phase locking, this effect can greatly enhance the conversion.

Nonlinear Dynamics

\section{Publications and Presentations}

Our nonlinear-dynamics effort encompasses several themes. We are especially interested in wave phenomena of all types, with an emphasis on geometrical methods such as symplectic geometry, Berry's phase and associated gauge structures, group theory, and catastrophe and bifurcation theory. Recent accomplishments include the development of quantization rules of the Bohr-Sommerfeld type for coupled wave equations, the extension of the rules of classical radiometry from geometrical to physical optics, and the classification of normal forms for linear mode conversion in coupled wave equations. There are applications for the results of this work in a variety of fields, including plasma physics, astrophysics, optics, and atomic, molecular, and nuclear physics.

\section{Theory}

A.J. Brizard, “New variational principle for linearized gyrokinetic Maxwell-Vlasov equations," abstract for the 34th Meeting of the American Physical Society Division of Plasma Physics (Seattle, WA, 1992); LBL32594a.

A.J. Brizard, "Nonlinear gyrofluid description of turbulent magnetized plasmas," Phys. Fluids B 4, p. 1213 (1992), LBL-31294.

A.J. Brizard, "Phase-space formulations for the Vlasov-fluid and kinetic-MHD models," abstract for the Sherwood Theory Conference (Santa Fe, NM, 1992); LBL-31920a.

A.J. Brizard, D.R. Cook, and A.N. Kaufman, "Wave-action conservation for pseudoHermitian fields," LBL-32849; submitted to Phys. Rev. Lett. (1992).
D.R. Cook, A.N. Kaufman, and A.J. Brizard, LBL, and H. Ye, Institute for Fusion Studies, University of Texas, "Analytic derivation of the magnetosonic wave reflection field for ICRF heating in tokamak geometry," abstract for the Sherwood Theory Conference (Santa $\mathrm{Fe}, \mathrm{NM}, 1992)$; LBL-31919a.

D.R. Cook, A.J. Brizard, W.G. Flynn, J. Morehead, and A.N. Kaufman,

"Conservation of wave-action for noneikonal wave fields," abstract for the 34 th Meeting of the American Physical Society Division of Plasma Physics (Seattle, WA, 1992); LBL-32569a.

D.R. Cook, A.N. Kaufman, and A.J. Brizard, LBL, and H. Ye, Institute for Fusion Studies, University of Texas, Austin, TX 78712,

"Analytic theory of magnetosonic reflection by gyrobaliistic waves in tokamak geometry," LBL-32668; submitted to Phys. Rev. Lett. (1992). 
D.R. Cook, W.G. Flynn, J.J. Morchead, and A.N. Kaufman, "Phase-space action conservation for non-eikonal wave fields," L.BL-33045; submitted to Phys. Rev. Lett. (1992).

W.G. Flynn and R.G. Littlejohn, "A normal form theory of pairwise linear mode conversion," abstract for the Sherwood Theory Conference (Santa Fe, NM, 1992); LBL-31940a.

W.G. Flymn and R.G. Littlejohn, "Pairwise linear mode conversion in two and three spatial dimensions," abstract for the 34th Meeting of the American Physical Society Division of Plasma Physics (Seattle, WA, 1992); L.BL-3257()a.

C. Jarzynski, "A diffusion equation for energy in eigodic adiabatic ensembles," Physical Review (Dec. 15, 1992); LBL_-32799.

A.N. Kaufman, "New mathematical methods for linear plasma problems" (review lecture), abstract for the Sherwood Theory Conference (Santa Fe, NM, 1992); LBL-31965a.

A.N. Kaufman, A.J. Brizard, D.R. Cook, and H. Ye, "Gyroresonant reflection, conversion, and absorption of magnetosonic rays in tokamak geometry," abstract for the 34th Meeting of the American Physical Society Division of Plasma Physics (Seattle, WA, 1992); LBL-32571a.

R.G. Littlejohn and W.G. Flynn, "Semiclassical theory of spin-orbit coupling," Phys. Rev. A 45 (7697), 1992; LBL-31749.

R.G. Littlejohn and W.C. Flynn, "BohrSommerfeld eigenfrequencies and eigenfunctions for vector wave equations," abstract for the Sherwood Theory Conference (Santa Fe, NM, 1992); LBL-31939a.

R.G. Littlejohn and W.G. Flynn, "General linear mocte conversion coefficient in one dimension," LBL_-33010; submitted to Phys. Rev. Lett. (a992).

R.G. Littlejohn, LBL, and R. Winston, Enrico Fermi Institute and Department of Physics, University of Chicago, "Corrections to classical radiometry," LBL-33011; submitted to J. Op: Soc. Am. A (1992).

S. Weigert and R.G. Littlejohn, "Diagonalization of multicomponent wave equations with Born-Oppenheimer example," LBL-32949; submitted to l'hys. Rev. A (1992).

\section{Ion E Plasina Sources}

P. Chen, L. Pan, D. Ponce, A. T. Young, and K.-N. Leung, "Laser and spectroscopic diagnostics of an $\mathrm{rf}$ inductively coupled $\mathrm{H}^{-}$ ion source plasma," abstract for the 34 th Meeting of the American Physical Society Division of Plasma Physics (Seattle, WA, 1992); LBL-32568a.

J.W. Kwan, G.D. Ackerman, C.F. Chan, M.E. Cooper, W.S. Cooper, C. . deVries, K.N. Leung, W.F. Steele, R.P. Wells, and M.D. Williams, "Development of a $\mathrm{D}^{-}$surfaceconversion source for ITER neutral beam applications," abstract for the 34 th Meeting of the American Physical Society Division of Plasma Physics (Seattle, WA, 1992); LBL32572a.

J.W. Kwan, G.D. Ackerman, C.F. Chan, M.E. Stuart, W. S. Cooper, G.J. deVries, K.N. Leung, W.F. Steele, R.P. Wells, A. West, and M.D. Williams, "A D" surfaceconversion source for neutral beam applications," in Proceedings of the 6th International Symposium on the Production and Neutralization of Negative lons and Beams (Brookhaven National Laboratory, 1992); LBL-33117.

K.-N. Leung, D.A. Bachman and D.S. McDonald, "RF driven multicusp source for positive and negative ion beam production," abstract for the 19th IEEE International Conference on Plasma Physics (Tampa, FL, 1992; LBL-32014a.

K.-N. Leung, D.A. Bachman, D.S. McDonald, and A.T. Young, "Ion sources for cyclotron applications," in Procerdings of the 13th International Conference on Cyclotrons and their Applications (Vancouver, B.C., Canada, 1992); LBL-.32038.

K.-N. Leung, D.A. Bachman, and D.S. McDonald, "RF driven multicusp ion source for particle accelerator application," in Procidings of the European Particle Accelerator Conference (Berlin, Germany, 1992); L.BL-32041ה.

K.-N. Leung, "RF driven ion sources," in Procedings of the 12th International Conference on the Application of Accelerators in Research and Industry (Denton, TX, 1992); LBL-32499.

K.-N. Leung, D.A. Bachman, and D.S. McDonald, "Applications of $\mathrm{rf}$-driven multicusp ion sources," abstract for the 34 th Meeting of the American I'hysical Society 
Division of Plasma Physics (Seattle, WA, 1992); LBL-32573a.

K.-N. Leung, D.A. Bachman, C.F. Chan, and D.S. McDonald, "An rf driven $\mathrm{H}^{-}$ion source and a low energy beam injection system for RFQ operation," in Procecdings of the 15th International Conference on High Energy Accelerators (Hamburg, Germany, 1992); LBL-32593.

K.-N. Leung, D A. Bachman, and D.S. McDonald, "RF driven multicusp source for pulsed or steady-state ion beam production," in Proceetings of the 9th International Conterence on Inn Implantation Technology (Gainesville, FL, 1992); LBL-32816a.

K.-N. Leung, D.A. Bachman, and D.S. McDonald, "Production of $\mathrm{H}^{-}$ions by an $\mathrm{rf}$ driven nulticusp source," in Procedings of the 6 th International Symposium on the Production and Neutralization of Negative lons and Beams (Brookhaven National Laboratory, 1992); LBL-33119.

D.S. McDonald, D. Bachman, P. Chen, C.Y. Li, A.T. Young, and K.-N. Leung, "Langmuir probe and optical diagnostics of an inductively-coupled rf plasma," abstract for the 19th IEEE International Conference on Plasma Physics (Tampa, FL, 1992); LBL31887a.

D. $\mathrm{McD}$ nald, D.A. Bachman, and K.-N. Leung," nhancement of $\mathrm{H}^{-}$production in an rf-driven multicusp source," abstract for the 34th Meeting of the American Physical Society Division of Plasma Physics (Seattle, WA, 1992); LBL-32574a.

A.T. Young, G.C. Stutzin, P. Chen, W.B. Kunkel, and K.-N. Leung, "Measurement of atomic and molecular hydrogen in a tandem magnetic multicusp $\mathrm{H}^{-}$ion source by VUV spectroscopy," Rev. Sci. Instrum. 63,4 (April 1992); LBL-31321.

A.T. Young, D.A. Bachman, P. Chen, K.-N. Leung, C.Y'. Li, and D.S. McDonald, "Spectroscopic and laser diagnostics of an inductively-coupled $\mathrm{rf}$ discharge $\mathrm{H}^{-}$ion source," in Proce'edings of the 4th NPB Technical Symposium (Argonne, IL, 1992); LBL-31995a.

A.T. Young, K.J. Bertsche, D.J. Clark, K. Halbach, W.B. Kunkel, K.-N. Leung, and C.Y. Li, "Design of a compact permanent magnet cyclotron mass spectrometer for the detection and measurement of trace isotopes," in Procerdings of the 13th International Conference on Cyclotrons and their Applications (Vancouver, B.C., Canada, 1992); LBL-32036a.

A.T. Young, M.D. Van Loy, and K.-N. Leung, "Electron quantum yield measurements from a laser-excited barium photocathode," abstract for the 34th Meeting of the American Physical Society Division of Plasma Physics (Seattle, WA, 1992); LBL-32576a.

A.T. Young, D.A. Bachman, P. Chen, K.-N Leung, C.Y. Li, D.S. McDonald, L. Pan and D. Ponce, LBL, and H. F. Döbele, Institut für Laser und Plasmaphysik, Universität $\mathrm{GH}$ Essen, Germany, "Laser, spectroscopic, and Langmuir probe diagnostics of an inductively-coupled $\mathrm{rf}$ discharge $\mathrm{H}^{-}$ion source," in Proceddings of the 6th International Symposium on the Production and Neutralization of Negative lons and Beams (Brookhaven National Laboratory, 1992); LBL-33118.

A.T. Young, C.Y. Li, C.F. Chan, and K.-N., Leung, "Development and application of a $\mathrm{C}$ ion source and extraction system to a high resolution mass spectrometer system," in Procerdings of the 6th International Symposium on the Production and Neutralization of Negative Ions and Beams (Brookhaven National Laboratory, 1992); LBL-33173.

\section{Beam Physics \& Technology}

O.A. Anderson, C.F. Chan, K.-N. Leung, and R.P. Wells, "Compact, cesium-free, $\mathrm{H}^{-}$ source and LEBT for RFQs," in Procedtings of the 4th NPB Technical Symposium (Argonne, IL, 1992); LBL-31937.

O. A. Anderson, "Emittance growth rates for decentered beams," in Proce'tings of the 4th NPB Technical Symposium (Argonne, IL, 1992); LBL-32012a.

O. A. Anderson and L. Soroka, "Envelope code for electrostatically accelerated beam with ESQ focusing," LBL-33246a (1992).

C.F. Chan and K.-N. Leung, "Improved lowenergy $\mathrm{H}^{-}$injector design for SSC," in Proceedings of the 4th NPB Technical Symposium (Argonne, IL, 1992); LBL-31992a.

C.F. Chan and K.-N. Leung, "Low-energy $\mathrm{H}^{-}$injector design for RFQ's" (unclassified), in Procestings of the 6th International Symposium on the Production and 
Neutralization of Negative lons and Beams (Brookhaven National Laboratory, 1992); LBL-33165.

C.F. Chan and M.C. Vella, "Design of a merging beamlet pre-accelerator for an electrostatic quadrupole accelerator (ESQ)," LBL-33369; submitted to Plasma Devices and Operations (Russia), 1992.

C.Y. Li, M. Cooper, K. Halbach, W.B. Kunkel, K.-N. Leung, R.P. Wells, and A. T. Young, "A permanent magnet system for a cyclotron used as a mass spectrometer," in Proceddings of the 13th International Conference on Cyclotrons and their Applications (Vancouver, B.C., Canada, 1992); LBL-32037a.

B. Shrauner, Department of Electrical Engineering, Washington University, St. Louis, MO, and O. A. Anderson, LBL, "Ion sheet beams with parabolic density profiles," Phys. Fluids B (August 1992); LBL-31088.

M.C. Vella, "Operability of an electrostatic quadrupole Accelerator with finite emittance beam," LBL-33071; submitted to Nucl. Instrum. Meth. (1992).

M.C. Vella, "Acceptance of a $200 \mathrm{kV}$ electrostatic quadrupole accelerator (ESQ)", LBL-33368 (1992).

R.P. Wells, O.A. Anderson, C.F. Chan, W.S. Cooper, G.J. deVries, J.W. Kwan, W.F. Steele, and M.C. Vella, "A proof-of-principle accelerator experiment for high energy neutral beams," abstract for the 34th Meeting of the American Physical Society Division of Plasma Physics (Seattle, WA, 1992); LBL32575a.

\section{Materials Modification}

A. Anders, S. Anders, and A. Förster, "Ion formation in vacuum arc cathode spots," abstract for the 19th IEEE International Conference on Plasma Physics (Tampa, FL, 1992); LBL-31980a.

A. Anders, S. Anders, A. Förster, and I. G Brown, "Pressure ionization: its role in metal vapour vacuum arc plasmas and ion sources," accepted by Plasma Sources Science and Technology (1992); LBL-32943.

A. Anders, S. Anders, B. Jüttner, and I. G. Brown, "Time dependence of vacuum arc parameters," LBL-33052; submitted to IEEE Trans. Plasma Sci. (1992).
A. Anders. S. Anders, and I.G. Brown, LBL, and P. Chow, Superior Vacuum Technology, Eden Prairie, MN, "Joining of metal films to carbon-carbon composite material by metal plasma immersion ion implantation," abstract submitted to the 1993 Spring Meeting of the Materials Research Society (San Francisco, CA, 1993); LBL-33164a.

S. Anders, A. Anders, K.M. Yu, X.Y. Yao, and I.G. Brown, "Vacuum arc generated thin films with reduced macroparticle contamination," 15th International Symposium on Discharges and Electrical Insulation in Vacuum (Darmstadt, Germany, 1992); LBL-31763.

S. Anders, A. Anders, J. B. Kortright, K.M. Yu, I.G. Brown and I.C. Ivanov, "X-ray mirror production by vacuum arc deposition techniques," abstract for the International Conference on Metallurgical Coatings and Thin Films, ICMCTF-93 (San Diego, CA, 1993); LBL-32988a.

I.G. Brown, "Vacuum arc ion sources for particle accelerators and ion implantation," invited paper, 15th International Symposium on Discharges and Electrical Insulation in Vacuum (Darmstadt, Germany, 1992); LBL32029a.

I.G. Brown, A. Anders, S. Anders, X. Yao, and K.-M. Yu, LBL, and I.C. Ivanov, Charles Evans and Associates, Redwood City, CA, "Plasma synthesis of thin metallic and composite films with atomically mixed substrate bonding," abstract for the 8th International Conference on lon Beam Modification of Materials (Heidelberg, Germany, 1992); LBL-32093a.

I. G. Brown, "Metal ion implantation for large scale surface modification," invited paper, 39th Symposium of the American Vacuum Society (Chicago, IL, 1992); LBL32203.

I.G. Brown, A. Anders, S. Anders, M.R. Dickinson, I. Ivanov, R.A. MacGill, X. Yao, and K.-M. Yu, "Metal plasma immersion surface modification: a new processing technique," Coatings for Advanced Heat Engines Workshop (Monterey, CA, 1992); LBL-32718.

I.G. Brown, "Development of a very large scale metal ion implantation facility," Coatings for Advanced Heat Engines Workshop (Monterey, CA, 1992); LBL-32743. 
I.G. Brown, "Plasma synthesis of thin films and multilayers with tailored atomic mixing," invited paper, TMS Conference on Plasma Synthesis and Processing of Materials (Denver, CO, 1993); LBL-33060.

I.G. Brown, "lon implantation doping techniques for rare-earths," abstract submitted to the Spring Meeting of the Materials Research Society (San Francisco, CA, 1993); LBL-33061a.

Z. Feng, M.A. Brewer, I.C. Brown, K. Komvopoulos, and D.B. Bogy, "Growth of diamond films on various carbon coatings with microwave plasma enhanced CVD," LBL-32744.

R.A. MacGill, X. Y. Yao, R. A. Castro and I.G. Brown, "Plasma treated screws for ultra high vacuum application," LBL-32906; submitted to ]. Vac. Sci. Technol. (1992).

R.A. MacGill, X.Y. Yao, R.A. Castro, M.R. Dickinson, and I.G. Brown, "Poor man's scratch tester," LBL-33033; submitted to J. Vac. Sci Techol. (1992).
J. Sasaki, K. Sugiyama, X. Yao, and I. Brown, "Multiple-species ion beams from titanium-hafnium alloy cathodes in vacuum arc plasma," LBL-33034.

X. Yao, A. Anders, S. Anders, I.G. Brown, M.R. Dickinson, and R.A. MacGill, "Plasma guns for the generation of pulsed metal plasma streams," abstract for the 19th IEEE International Conference on Plasma Physics (Tampa, FL, 1992); LBL-31820a.

X. Yao, K.-M. Yu, I.G. Brown, T.R. Devine, Jr., C. Kumai, and I.C. Ivanov, "Effect of titanium ion implantation on pitting corrosion behavior of aluminum," abstract for the 8th International Conference on Ion Beam Modification of Materials (Heidelberg, Germany, 1992); LBL-32156.

X. Yao, l.G. Brown and M.D. Rubin, "Modification of the optical spectra of glass by metal ion implantation," abstract for the 8th International Conference on Ion Beam Modification of Materials (Heidelberg, Germany, 1992); LBL-32176a. 

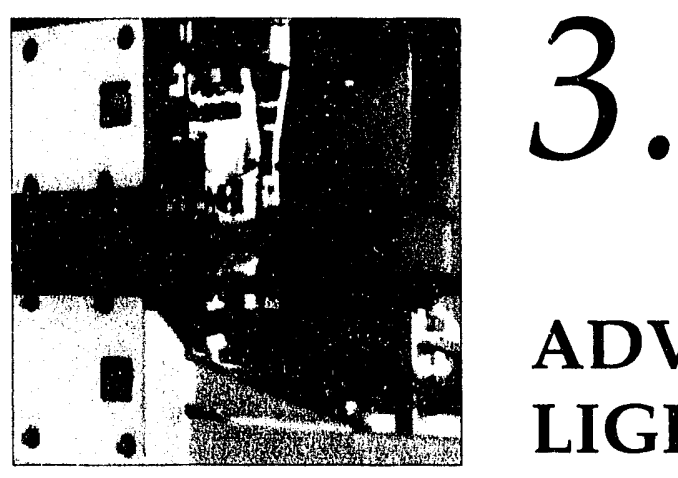

\title{
ADVANCED LIGHT SOURCE
}

\begin{abstract}
A S SIX YEARS' WORK COMES TO FRUITION, commissioning in spring 1993 remains the goal of the Advanced Light Source project. The ALS, bellwether of the third generation of synchrotron-radiation sources, is expected to circulate the first beam in the storage ring early in 1993. This milestone follows extensive work to commission and then gain operational experience with the injection linac and booster synchrotron-a process that will soon be re-enacted with the storage ring. The goal of these exhaustive commissioning activities is "push-button" operation, if not literally, then at least metaphorically: a comprehensive understanding of the machine's performance that will optimize service to users.
\end{abstract}

\begin{tabular}{|c|c|}
\hline $\begin{array}{l}\text { F. V'oelker* } \\
\text { NI. Wollet }\end{array}$ & $\begin{array}{l}\text { K. Bolin } \\
\text { I. Buchanan }\end{array}$ \\
\hline Experimental Systems & R. Cinclelurio \\
\hline B. Kincais & Ci. Carmignani \\
\hline c. Cork" & M. Chin \\
\hline E. Greene & T. Downs \\
\hline E. Ciullikson & F. Duarte \\
\hline k. Halbach* & M. Filmie \\
\hline W. Hassemolhl & D. Fong \\
\hline I. Heimann & Ci. Ciabor \\
\hline M. Howells & R. Cinsolway \\
\hline 2. Hussuin & K. Ciersisoni \\
\hline s. Irick" & A. Ciever \\
\hline $\mathrm{N}$. Iskunder & F. Henson \\
\hline C. hor & J. Hinkson \\
\hline 1. Le'e' & B. Holmes \\
\hline 1). $\log (x)$ & T. Jacksin \\
\hline S. Marks & J. lohnston \\
\hline W. Nckinney & I. fordan \\
\hline M.E. Melc/er & J. Julian \\
\hline R.C.C. Perera & A. Kruser \\
\hline D. I'helan & C.C. L \\
\hline R. Salow" & K. L..uchini \\
\hline R. Schluteter & 1. Lut \\
\hline M. Shlezinger & S. Magyiary \\
\hline S. Turek & P. Molinari \\
\hline I. Valder & M. Nolun \\
\hline A. Warwich & D. ()dicather \\
\hline f. Young & F. (Htens \\
\hline & D. lectersen \\
\hline Electrical Systems* & N. Photen \\
\hline 11. L.ancinter & G. Hortmann \\
\hline B. Bailey & (i. Potter \\
\hline K. Baptiste & 1. Reginatu \\
\hline 1. Bishop & ㅅ. Ritchie \\
\hline
\end{tabular}

Project Management

B.M. Kincaid (atter september 1942)

J.N. Mars funtil September 1992

P. Johnown*

A. (Teroll"

Scientific Program

Development

A. Schlachter

Accelerator Systems

A. lackion

R. Alvis

1. Bengtso(u)

R. Brokloff

H. Collims

E. Forest

C. Hauck

R. Killer

C. $\mathrm{Kim}$

(.) T.unterertion

Vansolett1

W. Neddah

R. Niller

H. Nishimura

Engineering Division and Plant Engineserme Department

* Environment. 1 lealth and Sittety Divinion

teonstruction and Naintenance Deportment and contract emplosedes

"Technical heformation Department

\begin{tabular}{|c|c|c|}
\hline C. Ritscher & T. Chan & 1. Swatin \\
\hline F. Rogers & J. Chin & 1. Taltabe \\
\hline A. Robls & M. Coleman & I.. Fiten \\
\hline L. Slyerriffe & C. Cummings: & J. Taylur \\
\hline J. Sopher & D. DiCiennars & J. Thompuson \\
\hline Ci. Stover & R. Duarte & C. Threatgill \\
\hline M. Szajbler & E. Fong & 1. Vertrees \\
\hline B. Tayleor & K. Franck & R. Wampler \\
\hline C. Timossi & 8. Ciee & W. Womi \\
\hline M. Whisenhunt & 1. Citligli & D. Yee \\
\hline K. Worlfe & 1. Henderson & N. Y'u \\
\hline R. Yadama & S. Hermandes & Conventional Facilities* \\
\hline 1. Zelver & E. Hoyer & I. I'ickrell \\
\hline J. Zukowski & D. Humphries & R. Baker \\
\hline Electricianst & R. k:“ns & L. Chan \\
\hline D. Alams & C. Inlenes & W. Lopes \\
\hline K. ciray & K. Kennedy & J. Pinle \\
\hline T. Nullarky & N. Kritscher & B. Sxhleifur \\
\hline W' Nelsom & T. Laturitzen & R. Scutero \\
\hline G. Petersien & C. Lawrence & R. Shilling \\
\hline 1. Phillips & J. 1..ax & T. Yutull \\
\hline G. Reis & $\begin{array}{l}\text { 1. Lomal } \\
\text { R. Low }\end{array}$ & Administrative Support \\
\hline D. Sandler & W. L.w & E. Atkin-()rvis \\
\hline I. Silnule, & l'. Luft & S. Butler \\
\hline Mechanical Systems ${ }^{*}$ & B. Macciill & 1.. Finch \\
\hline A. Pinterson & C. Matuk & S. Fujimura \\
\hline J. $A \mathrm{krc}$ & J. Nerneghelti & A. King \\
\hline N. Andresen & T. Nakale & 1. Konn \\
\hline (i. Andromaco & 1. Osborn & J. MacDomald \\
\hline B. Nivery & D. Plate & K. Rỵlands \\
\hline A. Black & D. Reimers & N. Talcott \\
\hline 1. Brown & M. Reimers & A. Tidwell \\
\hline J. Carrieri & K. $R e^{+}$ & Ci. Ureta \\
\hline B. Caylur & N. Sestrls & (i. V'ierra \\
\hline
\end{tabular}


The scientific programs of those users continued taking shape as well. The high brightness of the ALS beams will enable forefront research in a variety of scientific disciplines. Anticipating high demand for the ultraviolet and $x$-ray beams from the ALS, we are placing major empinasis on creating a "user-friendly" research environment and on being ready to launch an exciting research program.

As Figure 3-1 shows, the ALS consists of an electron source, a linear accelerator, a booster synchrotron, and a low-emittance storage ring. The storage ring has 12 long straight sections, 10 of which can accommodate insertion devices. Additionally, there are provisions for 48 radiation ports at the bend magnets, which also produce synchrotron radiation. Of these, 24 will be available for initial development.

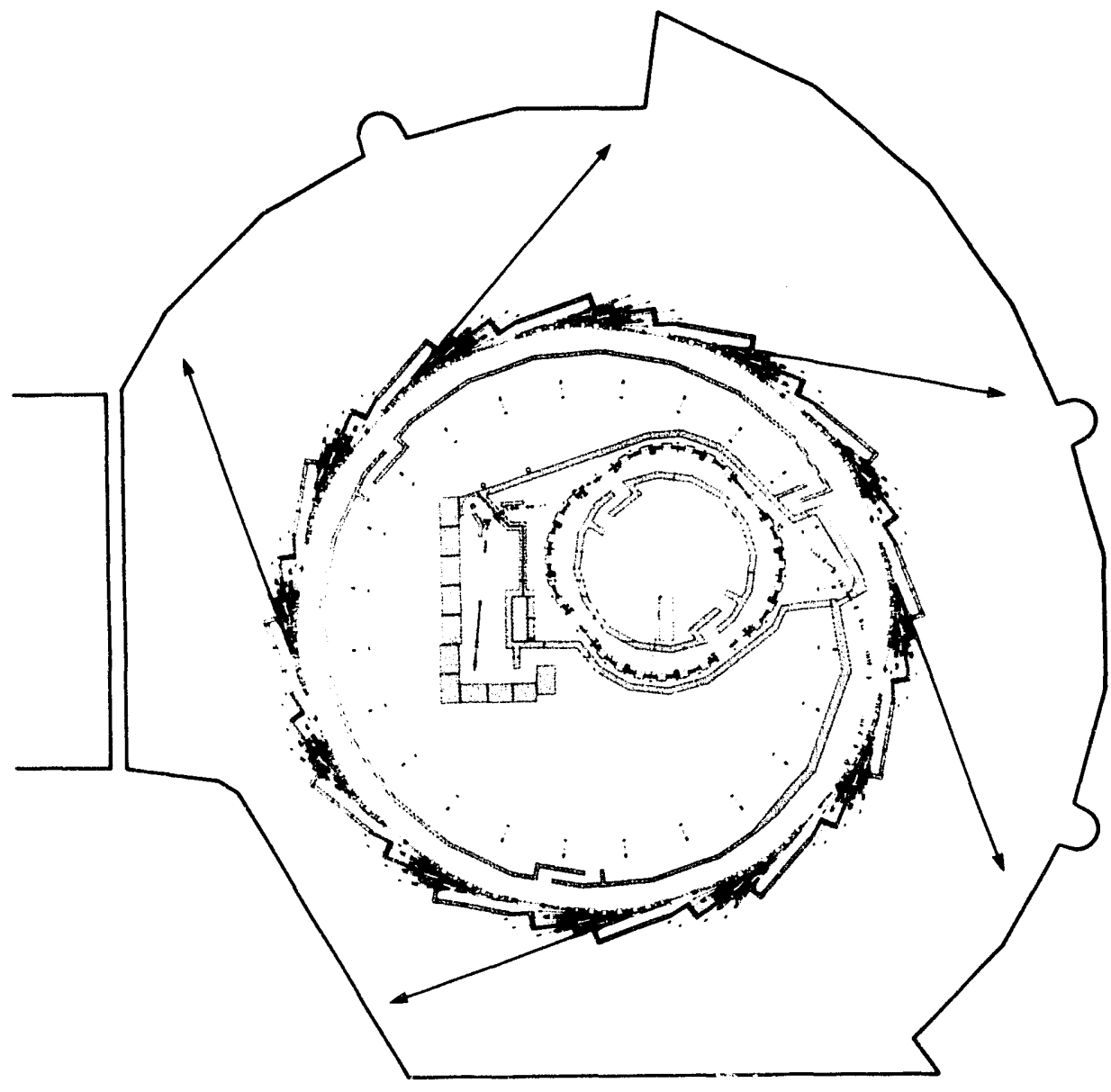

XBL $881 \cdot 8810$

In the year and $a$ half since the ALS building (Figuire the contractor, vast progress has been made within. The injector complex (linac and booster synchrotron) has been commissioned, and the final touches are being put on the storage ring. Throughout these activities, extensive testing and verification work was performed under the comprehensive quality-assurance policies of the ALS project. ALS environment, safety, and health personnel are also overseeing the commissioning process and preparing for user-facility operations.
Figure 3-1. In the ALS, a linac and a booster synchrotron inject electron bunches into a storage ring. The multiple straight sections in the storage ring can each accommodate an insertion device to enhance production of synchrotron light from the verylow-emittance electron beam; the bend magnets also produce useful synchrotron light.

\section{Construction Progress}


74

4

.8

4

저 
A major feature added midway through the project-a thermal stabilization system for the building-is in progress and should be complete in early 1993. It was added in response to a 1988 study, the first-ever systematic analysis of how ambient temperature variations could affect the performance of a synchrotron light source by making components expand and contract. The study indicated that temperature stabilization to within $\pm 1^{\circ} \mathrm{C}$ would be necessary to avoid shifts in beam position. This system will air-condition the storage-ring tunnel and the experimental area outside it.

The ALS injection complex ans commissioned in the early months of 1992. Noze the final touches are being put on the stornge ring as we prepare to inject a coasting beam in early 1993, then add the ability to store the beam and move on with the ring's commissioning.

The injection system ( $50-\mathrm{MeV}$ linac and $1.5-\mathrm{GeV}$ booster synchrotron) was entirely commissioned in spring 1992. The full design energy of $1.5 \mathrm{GeV}$ was achieved in January, and a 1.5-GeV beam at a current of $14 \mathrm{~mA}$, which is sufficient to fill the storage ring in a few minutes, was extracted from the booster in April. (The full design value is $16 \mathrm{~mA}$.) This prompt success is a testimony to the craftsmanship that went into construction, assembly, installation, and alignment.

in order to consolidate and build upon these achievements, we have been taking every opportunity to operate the injection system and have also begun a detailed study of its beam dynamics. These activities have enhanced our understanding of these complicated systems, providing knowledge that will provide continuing benefits to users, such as improved beam stability and smoother, more-reliable operations.

Examples of this work included improvements to the beam's energy stability and energy spread in the linac, which, in turn, improved the efficiency of beam transfer from the linac to the booster. Previously, voltage ripples in the rf modulators, as well as electron-gun timing jitter, had caused considerable linac beam-energy jitter.

We also worked on a problem called "beam loading" in which the passage of the intense beam depletes the rf energy in the linac. This depletion reduces the effective accelerating gradient experienced by the following electron bunches, increasing the beam's energy spread and therefore reducing the booster's beam-capture efficiency. To compensate, we are making the effective accelerating gradient in the linac increase during a period of some $100 \mathrm{~ns}$ by changing the rf phase. We have found that this approach can reduce the energy spread to a tolerable level and are continuing a detailed study.

Recently we added single-bunch-mode capability to the gun electronics. In the single-bunch mode, the gun produces one bunch, containing up to $2.4 \times 10^{10}$ electrons, with a duration of $2 \mathrm{~ns}$ (full width at half maximum). This capability will enable single-bunch operation of the storage ring, which is useful for certain experiments.

This new mode also proved useful for studying the dynamics of the beam in the bunching system and in the linac. The bunching system compresses the 2-ns bunch coming out of the gun by about a factor of 100 . Because of the pulse durations involved-about 20 ps at the linac exit-the

\section{Accelerator Assembly and Commissioning}


exact compression is hard to measure directly with presently available diagnostics. However, the pulses inevitably experience some energy spread caused by the sinusoidal nature of the accelerating fields. By measuring the energy spread, which is easier to do, we can infer the pulse length and use this information to further tune the bunching system.

Other beam-dynamics studies focused on nonlinearity correction in the booster. Even if every electron from the linac could be injected perfectly, the electrons would oscillate transversely around the closed (design) orbit because of subtle magnet misalignments. The misalignments distort the closed orbit, which otherwise would be perfectly at the center of the vacuum chamber. Beam losses could be very large if the amplitude of the betatron oscillations, a type of movement the beam undergoes, were also large.

To solve this problem, we use a system of 32 beam-position monitors and 32 corrector magnets. Thus closed-orbit distortions are measured and corrected to a ievel that does not usually cause any noticeable beam losses unless the tune meets certain resonance conditions." However, these tunes are expected to shift while we "ramp up" the booster to its full energy. The ramp-up process includes an increase in the fields of the dipole (bend) and quadrupole (focu is) magnets. The ratio of these fields does not stay constant, owing to the remanent field in the magnet cores at the beginning of the ramp and to core saturation near the end of the ramp. These nonlinear fields were measured during magnet production, and provisions were made in the control system to program the quadrupole magnet strengths to correct for the tune shifts. We are in the process of perfecting this technique, called "linearity correction," by measuring the tunes and making fine adjustments.

The last physical piece of the injection system was finished in 1992: a transfer line from the booster to the storage ring, along with the requisite extraction and injection elements.

While the injection complex was being commissioned, fabrication and installation of storage-ring components continued apace. By April 1992, after a planned delay to cllow trucks to deliver the shielding blocks, the last of the curved arc sections, or "sector chambers," was installed. The 12 arcs are the vacuum chambers around which the bend and focus magnets are placed. The arcs are interspersed with 12 "straight sections" designed to accommodate insertion devices. Two straight sections are currently occupied, one for acceleration and the other for beam injection; in the other 10 locations "spool pieces," or straight lengths of ultrahigh-vac'sum pipe, are used as placehoiders. Survey and alignment continued to bf a critical activity throughout.

As installation proceeded, we continu ?d developing techniques for "baking out" the assembled arc sectors, st aight sections, and spool pieces at $120^{\circ} \mathrm{C}$ to drive off contaminants, enabling us to pump the ring down to ultrah igh vacuum. We also retrofitted additional mounting struts to control the rovement of the vacuum chambers, and additional heaters were instal'ed to control thermal gradients in the arc sectors.

Joining the arcs and the straioht sections presented interesting crossdisciplinary challenges in mechanical engineering and accelerator physics. Figure 3-3 shows the transition area. The vacuum bellows provide flexibility

\footnotetext{
The tune is the number of betatron uscillations the electrons undergo during one revolution around the svnchrotron.
} 

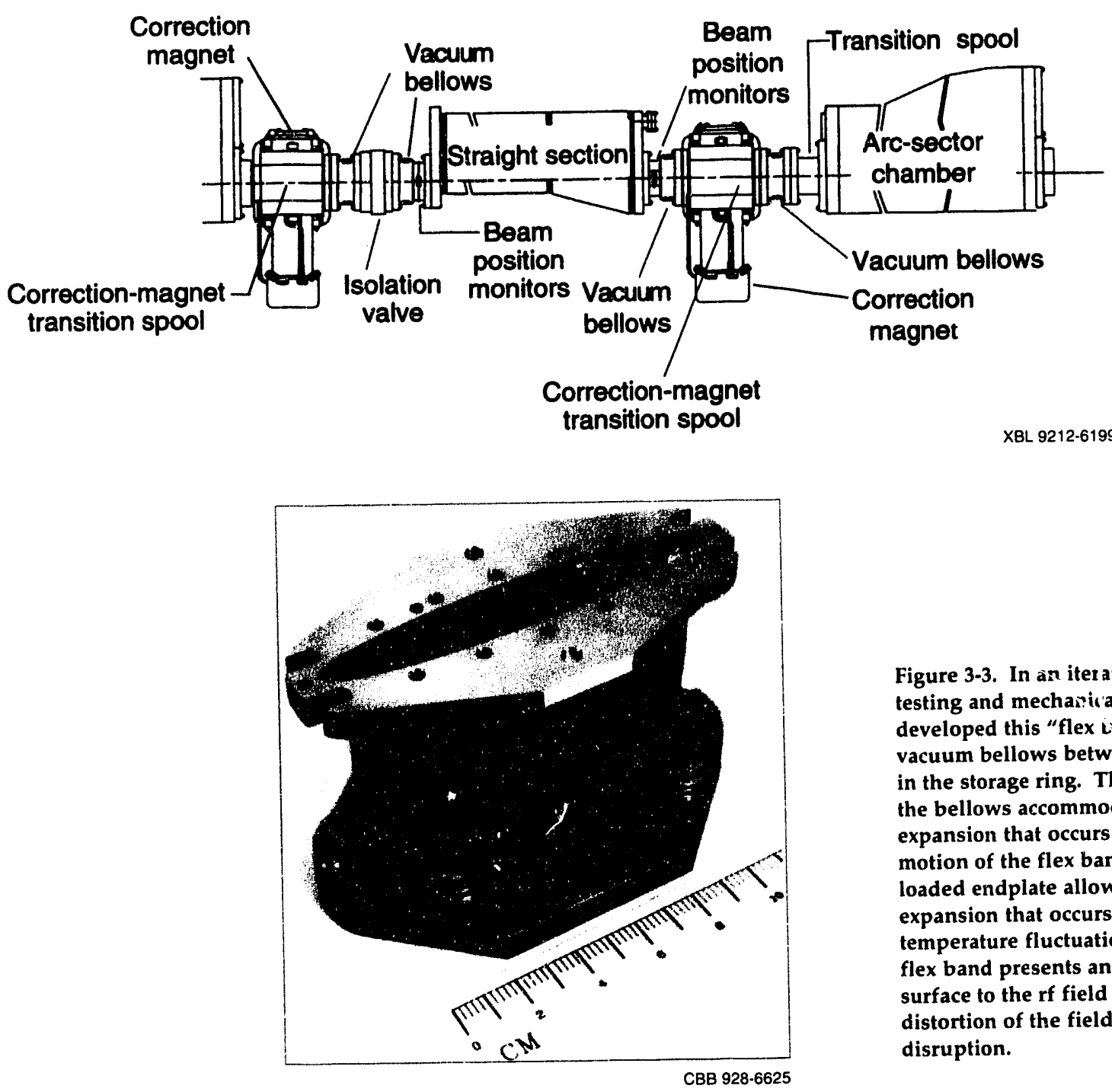

Figure 3-3. In an iter ative effort involving rf testing and mechariual design, we developed this "flex iand," an insert for the vacuum bellows between arcs and straights in the storage ring. The accordion pleats of the bellows accommodate the large expansion that occurs during bakeout, and motion of the flex band against the springloaded endplate allows for the small expansion that occurs during ambienttemperature fluctuations. Meanwhile, the flex band presents an effectively smooth surface to the rf field inside, thus avoiding distortion of the field and the ensuing beam disruption.

to accommodate the thermal expansion and contraction that occur during the bakeout cycle. However, their accordion shape would distort the rf field and consequently disrupt the beam. To solve this problem, we designed special "flex bands" to fit inside the bellows. The bands are made from rectangular pieces of beryllium-copper alloy that are cut through almost, but not quite, from end to end. The cuts allow for expansion during bake-out, yet the whole assembly appears smooth from an $\mathrm{rf}$ standpoint at normal operating temperatures. Each flex band is additionally spring-loaded at one end; this allows for the much-smaller dimensional changes that will occur in the normal operating temperature range of $20-28^{\circ} \mathrm{C}$. Combined with the "transition spools" that blend the diamond-shaped internal profile of the arcs into the elliptical profile of the straight sections, the flex bands will provide a smooth rf path throughout the ring. 


\section{Supporting Technical Facilities}

\author{
Control System
}

RF System

Instrumentation
Nearly all aspects of a modern accelerator are enhanced by electronic systems for measurement, control, and power. In 1992, as the injection complex was commissioned and preparation began for storage-ring commissioning, electronics work at the ALS became even more important.

Significant upgrades were made to the ALS control system, which in partial form has been used throughout the commissioning of the injection system. One of the highlights of this progress is a new, more-capable design for the Intelligent Local Controller (ILC). A key module in the distributed architecture of the control system, the ILC performs much of the processing at the controlled component. The new ILC was successfully tested, and 325 units have been assembled for use in the storage ring. The new design incorporates true 16-bit analog-to-digital and digital-to-analog converters, as well as four times as much memory; it runs $60 \%$ faster and consumes less power. An improved input/output scheme in the new ILC has obviated the need for more than 100 cards that would have interfaced ILCs with their serial link to the rest of the control system. Nineteen ninety-two also saw upgrades to the Collector Micro Module computer boards (now based on the 33-MHz 80486 microprocessor) and the acquisition of 12 more $50-\mathrm{MHz} 80486$-based personal computers for use as operator consoles, as well as a large effort in the development of new applications programs for the operator consoles in support of commissioning.

Throughout the year, the linac and booster rf systems have shown high reliability, operating on a regular basis for development, injector commissioning, and accelerator studies.

All components of the storage ring's high-power $500-\mathrm{MHz}$ rf system are now at LBL, and the major items-the power source, cooling systems, waveguide feeder network, and cavities-are now installed. Early in the year we successfully tested a cavity fitted with a tubular waveguide ceramic window, operating the cavity at $70 \mathrm{~kW}$ continuous duty (the power limit of the rf test rig). The normal operating power level of the cavity, with no beam, is $62 \mathrm{~kW}$. This success verifies the acceptability of a unique design meant to overcome the problems many other storage rings have had with rf windows. Individual testing of discrete components and subsystems of the rf system is well advanced.

Key roles are played in the control system by several types of instruments that determine the location and quality of the electron beam at various points in the injection system and storage ring. Among these instruments are the 32 beam-position monitors now fully operational in the booster, keeping tabs on the location of the beam as it is accelerated from $50 \mathrm{MeV}$ to $1.5 \mathrm{GeV}$ (nearly the speed of light) - a process that takes only about $1 / 3$ of a second, yet involves more than 1.3 million revolutions around the booster. One of the many things that could go wrong in this process is closed-orbit deviation, or distortion of the ideal orbit, caused by subtle misalignment of the magnets. The beam-position monitors store data gathered over as many as 1000 turns and feed it into the control system. Programs in the control system then perform fast Fourier analysis of the data and actuate 32 corrector magnets, which trim the orbit to compensate for these errors, thus keeping the beam well within the limited aperture of the vacuum chamber. 
Our attention has now turned toward the storage ring, which will have its own arrays of instruments to characterize not only the stored electron beam, but also the photon beams. Among the 1992 highlights was the fabrication of 100 electron-beam position monitors using mass-production techniques that have given us a state-of-the-art system at well below the budgeted cost; testing and installation is nearly complete.

Still under development is a longitudinal and transverse beam-damping system. This system uses a novel digital approach for the processing of data from the beam instrumentation in the storage ring, and is being developed jointly with the PEP-II "B-factory" effort at the Stanford Linear Accelerator Center. Further details of the extensive, collaborative PEP-II effort may be found in the Center for Beam Physics chapter of this report.

With the user program less than a year away, the experimental systems-insertion devices for enhanced production of synchrotron light and beamlines for delivering the light to user's experiments-are receiving a great deal of attention (See also the later section on "Interaction with the User Community.") Insertion devices-the $k e y$ to producing small, intense, tunable beams of synchrotron light-moved from design into fabrication, and three of them are near completion. Also critical are high-performance beamlines that convey the photon beams to the users' experimental stations without compromising the brightness of the beams; the components of these systems must maintain precision even under high heat loads. Six experimental beamlines, including two complete beamlines, the "front ends" for three more, and a diagnostic beamline, will make up the initial complement.

Based on a uniform generic design, the three undulators currently being funded by the ALS project are being fabricated Two undulators designated U5.0 (for their $5.0-\mathrm{cm}$ magnetic periods) are nearly complete. Fabrication of U8.0 is well under way; the components of the magnetic structure have been fabricated and assembled into periodic modules, which are being put together on the support structure.

Figure 3-4 shows the first U5.0 as it stands on the ALS floor, complete except for its vacuum system, which is now undergoing bakeout and vacuum test. Also shown is a detail of the magnetic structure, a highly precise assembly in which all the pole surfaces are aligned to within $50 \mu \mathrm{m}$ in both the horizontal and the vertical planes. In general, extremely tight mechanical and magnetic tolerances are required in order to fully realize the photon-beam brightness made possible by the low emittance of the electron beam in the ALS. Typical tolerances for the placement of magnetic materials in ALS insertion devices are $12-50 \mu \mathrm{m}$, and gap motion must be controlled and reproducible to about $1 \mu \mathrm{m}$. This precision must be achieved and maintained despite magnetic loads that can reach almost 40 metric tonshence the massive construction seen throughout.

The 4.5-m-long undulators will generate high-brightness radiation at photon energies from $20 \mathrm{eV}$ to $2.5 \mathrm{keV}$ (that is, in the ultraviolet and soft- $x$ ray regions of the spectrum) in the fundamental and the third and fifth harmonics. The tuning range is determined by the length of the magnetic period, the peak magnetic field achievable, and the electron-beam energy. This radiation will be more than 10 times brighter than radiation from the

\section{Experimental Systems}

\author{
Insertion Devices
}


Figure 3-4. The undulator U5.0 is essentially complete. Not shown here is the prototype insertion-device control system that is now being tested on the assembly; nor is the recently completed vacuum chamber. Another U5.0 and a U8.0 are following it through the assembly process, which involves painstaking alignment and magnetic

characterization. The undulator gap is adjustable in order to

change the magnetic field and hence the wavelength of the synchrotron light. During this process, the position control must be accurate and reproducible to $\pm 1 \mu \mathrm{m}$ despite magnetic attraction of nearly 40 metric tons, hence the massive construction.
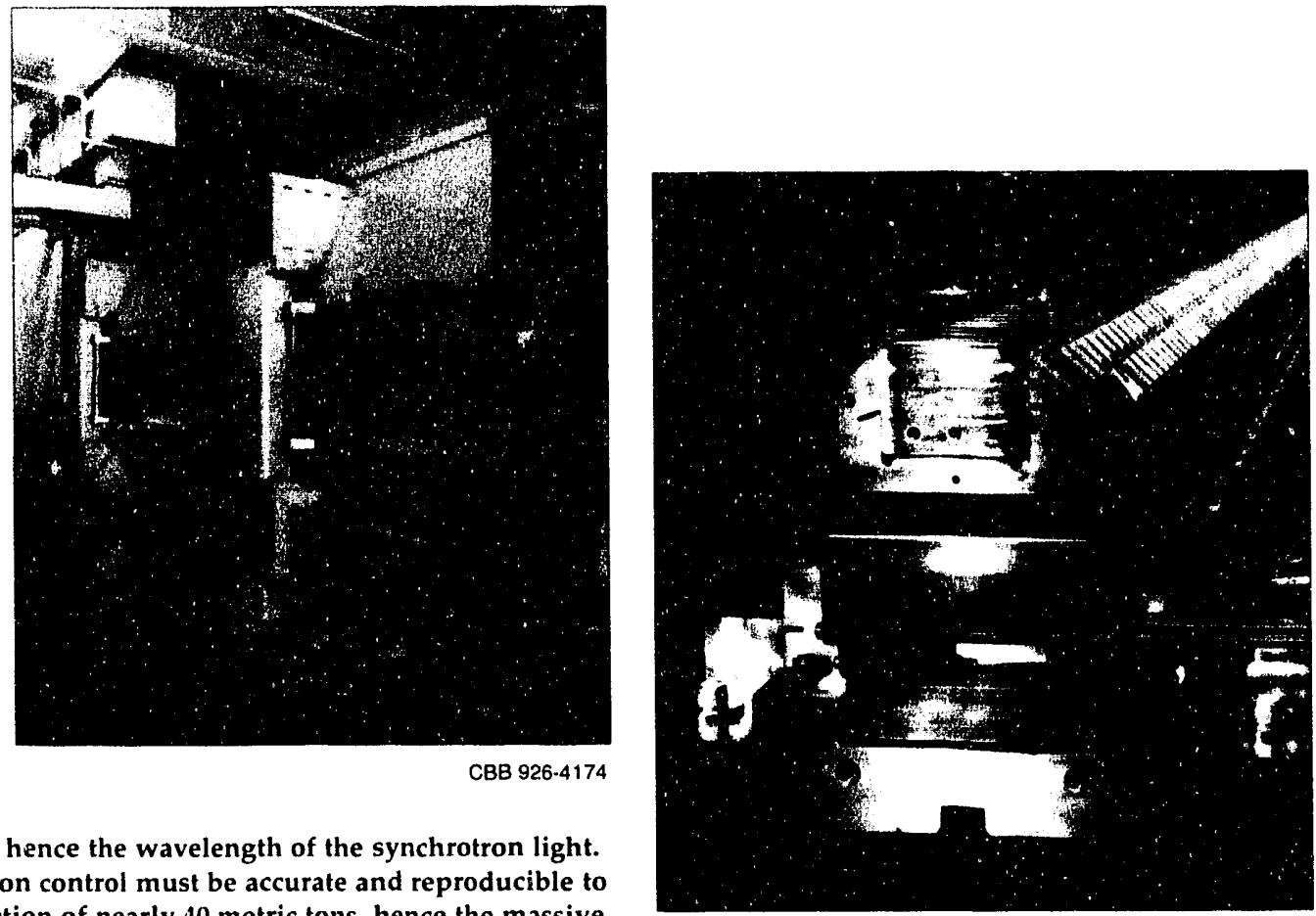

СвB $922 \cdot 1130$

best existing sources. Within this range, the photon energy will be tuned by varying the magnetic field, which changes as the gap between the magnetic poles is widened or narrowed.

The undulators are known as hybrids because the magnetic fields are produced by a high-strength permanent-magnet material, neodymium-ironboron, and the field seen by the electron beam is shaped by a ferromagnetic material, vanadium permendur. Each $U 5.0$ has 181 pole pairs and requires about 2100 magnetic blocks. The U8.0 now being built has 113 pole pairs and requires about 1400 blocks. By the time the magnetic structure is assembled, the blocks have already run a gauntlet of magnetic characterizations to verify their acceptability and to determine their optimum place in the magnetic structure. The assembled structures, after various mechanical measurements, are then characterized magnetically with a Hall-effect scanning system developed at LBL.

This system (Figure 3-5) traverses its 5.5-m path down the undulator in $20 \mathrm{~s}$, earning it the nickname "The Luge" after the sled used in the Olympics. Along the way, it takes 5500 measurements of the field inside the undulator and near the ends. Luge measurements to date show that U5.0 has met the $0.25 \%$ uncorrelated field-error tolerance $(0.23 \%$ was achieved throughout the device, no worse than $0.16 \%$ between each set of poles). This performance indicates that the undulator will indeed be capable of generating usable photon beams through the fifth harmonic, as predicted.

A prototype of the insertion-device control system is being tested on the completed U5.0. 


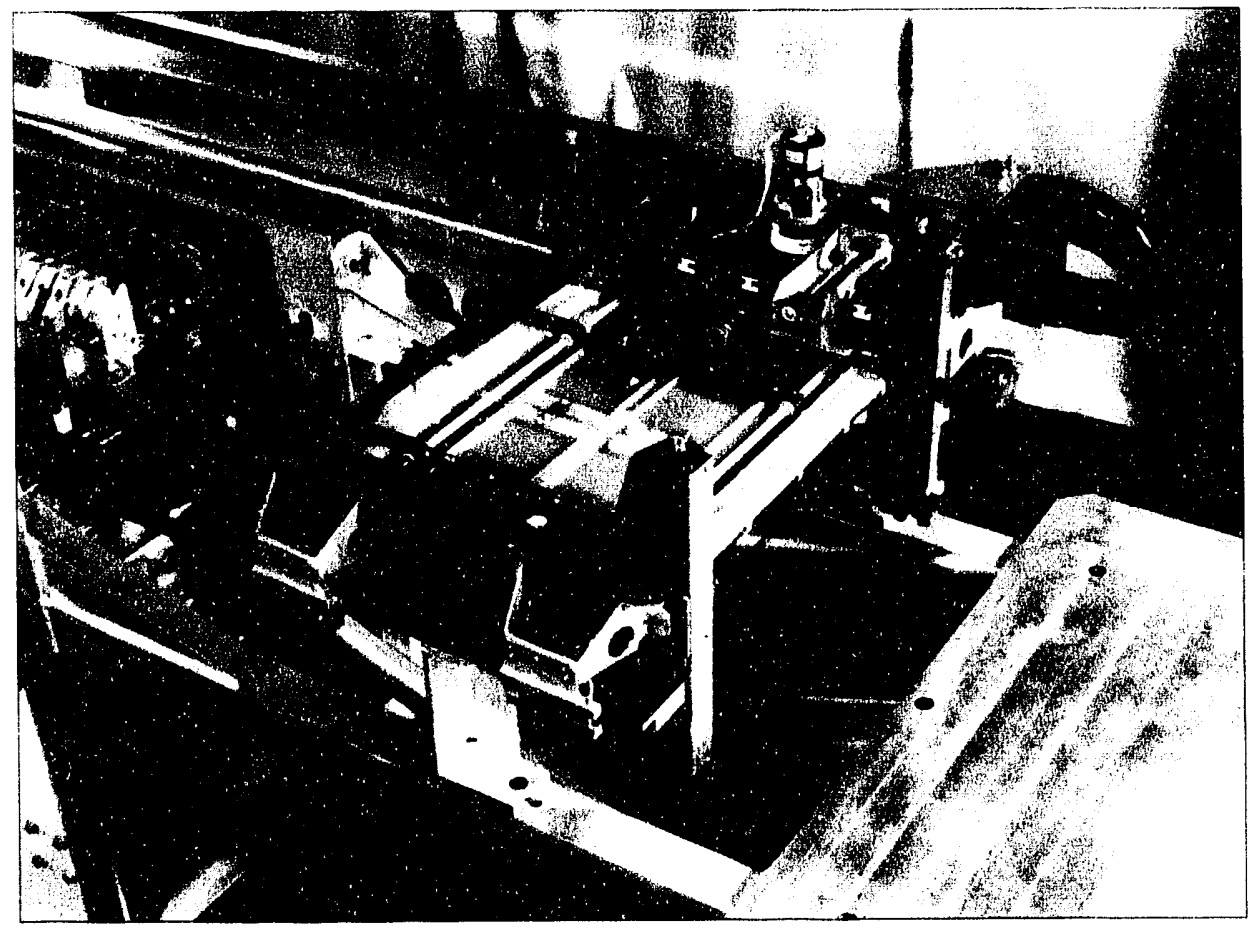

CBB 924.2877

Five beamlines will make up the first group of experimental systems to be installed at the ALS. (By "experimental systems" we mean everything up to and including the monochromator, as shown in Figure 3-6. The users' experimental apparatus lies beyond the monochromator.) Three beamlines will deliver undulator radiation from the U5.0 and U8.0 insertion devices; two more will deliver bend-magnet radiation. Figure 3-7 shows the initial complement of beamlines and their locations.

Of the three undulator beamlines, two are being built by the ALS, one for U8.0 and the other for one of the two U5.0 undulators. (Other beamlines will be provided by their users.) The U5.0 beamline will be dedicated to spatially resolved spectroscopy (spectromicroscopy) of surfaces, interfaces, and other physical systems; the U8.0 beamline is intended for the study of photoprocesses in atoms, molecules, and ions. Both will have sphericalgrating monochromators.

The small size of the ALS photon beams creates new challenges for beamline designers, as does the emphasis on insertion devices. First, the source size and divergence have become very small. For undulators at the high-photon-energy end of the spectral range, the rms photon-beam size is typically $330 \mu \mathrm{m}$ horizontal by $65 \mu \mathrm{m}$ vertical, and the rms divergence is typically $40 \mu \mathrm{m}$ horizontal by $30 \mu \mathrm{m}$ vertical. To avoid loss of light from this smal! source, tighter tolerances are needed for the figure and finish of relay optics and monochromator components." Further, it is now practical to achieve higher resolution by the use of narrower monochromator slits;

\footnotetext{
- Figure refers to the accuracy of the profile at low spatial frequencies or long periods, whereas surface finish refers to the smoothness of the profile at high spatial frequencies or short periods.
}

Figure 3-5. "The Luge," an LBLdesigned Hall-effect scanning system, moves the length of an undulator in $20 \mathrm{~s}$ while taking 5500 magnetic measurements. Data obtained in luge measurements on the first U5.0 indicate that the undulator has an uncorrelated field-error tolerance of $0.23 \%$ throughout its length and $0.16 \%$ between each set of poles. This performance exceeds the specifications and ensures that the higher harmonics (third and fifth) of the photon beam will be usable.

\section{Beamlines}


Figure 3-6. Beamline 7.0 (fed by one of the U5.0 undulators) typifies an ALS undulator beamline, with its LBL-designed spherical grating monochromator. This beamline will be devoted to spectromicroscopy with UV and soft-x-ray light.

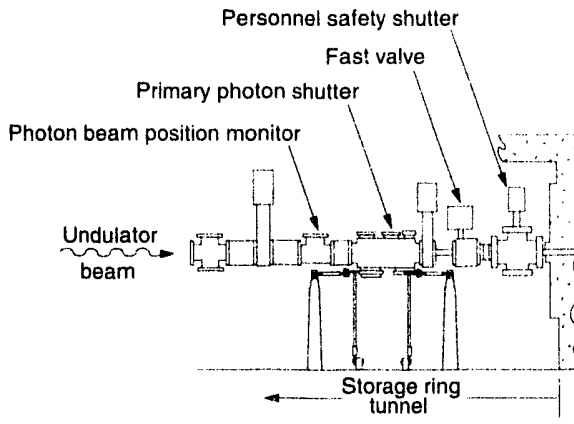

Beamline 7.0
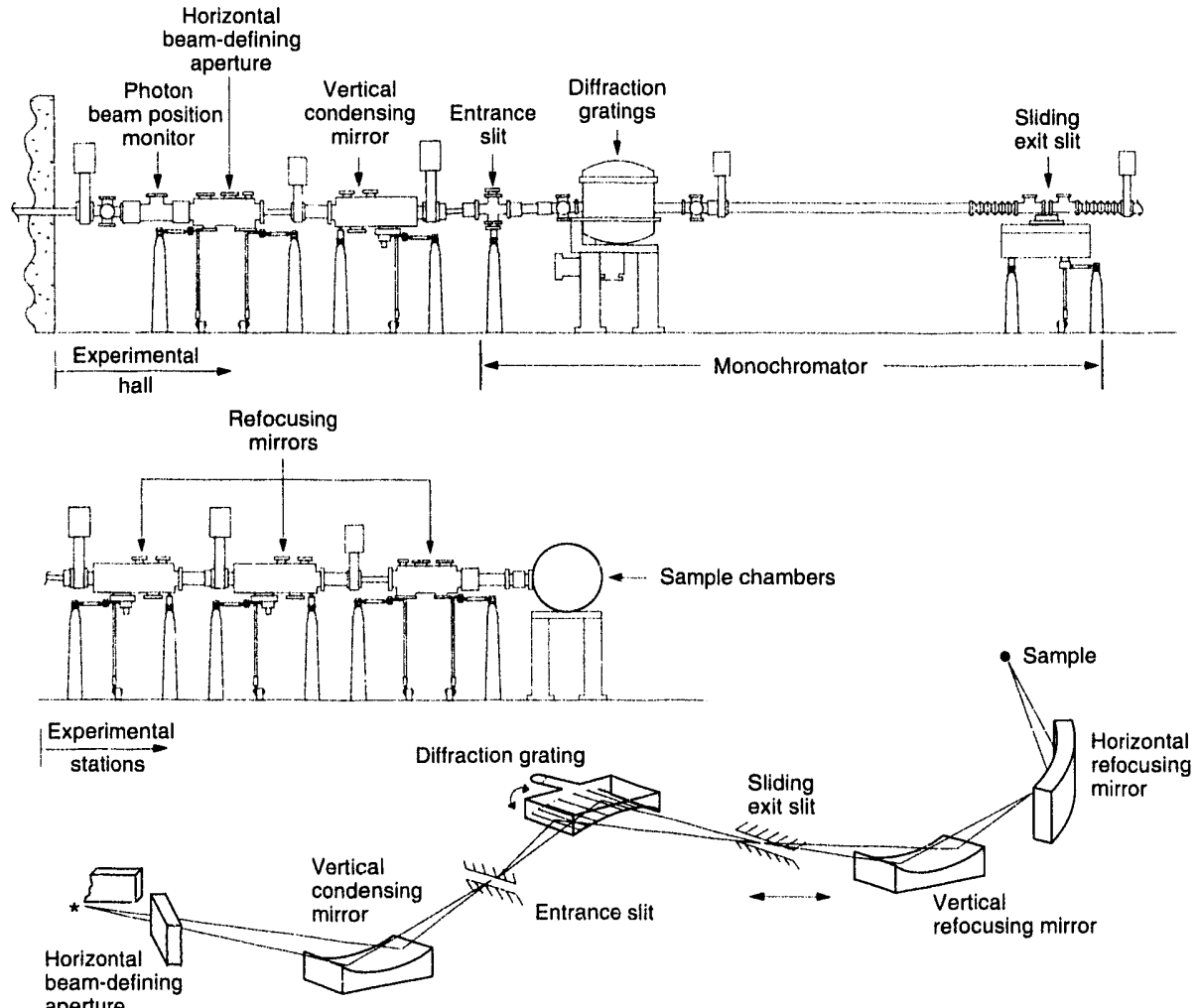

XBL 927-5322

therefore, monochromator components need tighter tolerances to avoid loss of resolution. Finally, the photon-beam power has increased to several kilowatts per square centimeter, implying that the optical components must hold their precision when a tiny area, and only that area, is continually illuminated with what is essentially a powerful x-ray laser. The resulting requirements for control of thermal distortion and stress complicate the design.

During 1992, we made significant progress with our design concepts for undulator beamline optical components, developing them into detailed specifications for fabrication and procurement. With about 15 mechanical engineers, designers, and drafters involved with beamline development, efforts have been primarily devoted to final design of hardware, as well as the subsequent stages of fabrication, procurement, assembly, and installation. As a result, most of the hardware is now being fabricated and will soon 


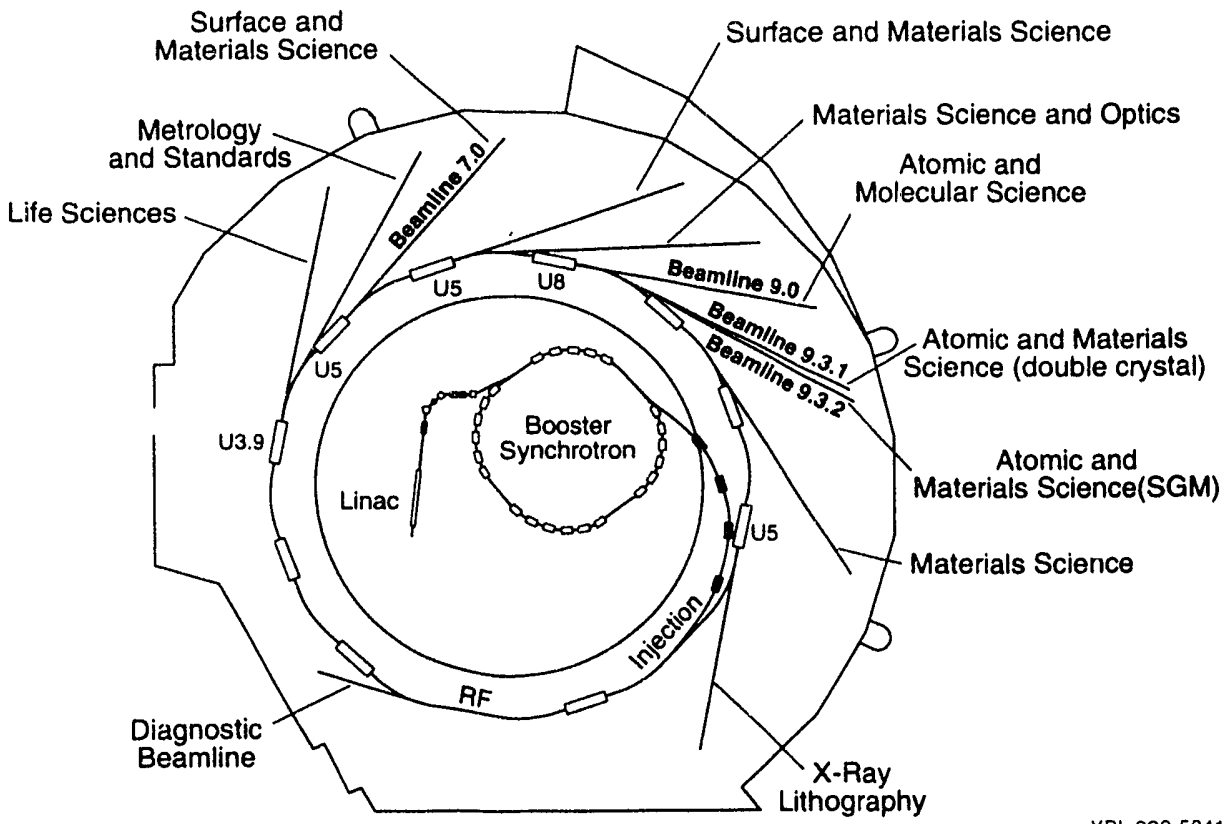

Figure 3-7. A diagram of the ALS floor shows the beamlines that are planned for construction through 1995. The numbering scheme for beamlines is related to the storagering sector where they begin. The photo gives a glimpse inside the storage-ring shielding, revealing the beamline "front ends" (covered, circular openings directly underneath tall structures). The first four from the left are for bendmagnet ports; the rightmost one is for an undulator and comes off the straight section immediately behind the arc sector shown in this photo. The tall structures are photon stops and their actuators; associated with each photon stop but hidden below the vacuum chamber is a titanium sublimation pump for immediate, local removal of photon-desorbed gases.

be ready for final assembly and installation. "Front-end" components for the undulator and bend-magnet beamlines have, for the most part, been assembled and tested. Some additional engineering work has been done to support user planning for future beamlines and installation of experimental facilities.

Fabrication of the directly cooled grating substrates for the two sphericalgrating monochromators was taken through the polishing stage, with the quality of surface figure and finish meeting all specifications. The grating blanks are now ready for the final ruling and coating operations. Water-cooled $x$ ray mirrors for these and other undulator

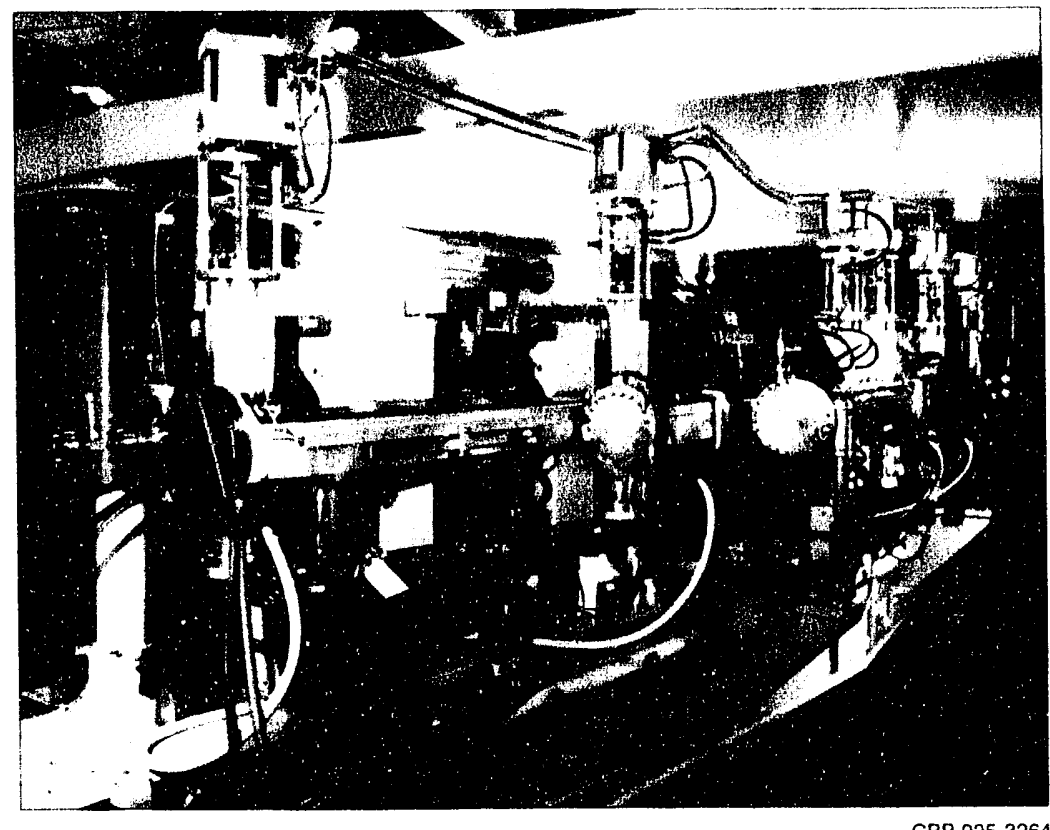

CBB $925-3264$ beamlines received considerable attention in 1992. Following extensive thermal analysis, vibration analysis, and prototype testing, the final design details were completed; parts are currently being fabricated for the mirror mounting and positioning systems. The mirrors themselves have been polished and meet all specifications for figure and finish, and are awaiting final optical coatings. A variety of other engineering and fabrication tasks also got under way.

In addition to building the two user beamlines, we plan to install a diagnostic beamline with which we can use synchrotron radiation for precision imaging and transverse measurement of the electron beam in the 
storage ring. (The ALS has a natural emittance of $3.4 \times 10^{-4}$ meter-radians and, in the first bend magnet, has horizontal and vertical beam sizes $\sigma_{h}$ and $\sigma_{v}$ of $44 \mu \mathrm{m}$ and $83 \mu \mathrm{m}$, respectively, assuming a 10:1 ratio of horizontal to vertical emittance. Simple diffractive optical calculations show that it is not feasible to image this beam with visible-light optics; imaging must be performed using photon energies greater than $50 \mathrm{eV}$.) This beamline will be used to provide 1:1 images of the electron beam in the storage ring, take transverse measurements of the beam, and obtain information about its positional stability and the length of the electron bunches of which it consists. The images and the data will enable the accelerator physicists to finetune the beam to achieve the ALS goals for very small source size. These measurements are essential for optimizing the operation of the storage ring, insertion devices, and monochromators. The diagnostic beamline will be installed at the first bend magnet downstream from the straight section used for acceleration. This constricted area of the experimental floor is the least desirable for user beamlines.

The beamline will have an imaging system for 200-eV photons and a "white beam" port (i.e., a port with no monochromator, passing the full spectrum of bend-magnet radiation) with a streak camera to obtain timing information. The imaging system will employ two crossed, spherical mirrors in a Kirkpatrick-Baez configuration to eliminate astigmatism. Use of 1:1 imaging will eliminate coma, thereby producing an image of the source limited only by the residual aberrations of the optics. Real-time imaging of the beam should be feasible with the use of a high-resolution charge-coupled device.

The design of the diagnostic-beamline imaging system for the ALS was implemented with an eye toward application in other third-generation synchrotron radiation sources, all of which will present challenges similar to these.

Optical Components and Characterization
One of the most important areas of beamline R\&D has been characterization of optical surfaces. The typical quality of optical components for use in synchrotron-radiation beamlines had been sub-optimal for many years. Our requirements are stringent (slope errors in the range of 0.5 microradian, for example), so optical metrology plays a key role. To this end, the ALS project funded the construction of two long-trace profilers based on a surfaceprofiling instrument developed at Brookhaven National Laboratory. One is operating in the new ALS Optical Metrology Laboratory, where it is housed in a class- 10000 cleanroom to avoid contamination of optical surfaces. The other is installed at Photon Sciences International, a private company with which we are cooperating to develop manufacturing techniques for watercooled metal beamline optics. These surface profilers make it possible for us to obtain the best beamline optics available while making significant contributions to state-of-the-art surface profiling.

To date, the profilers can measure surface slope error to better than 1 microradian rms. With the help of these instruments, water-cooled metal optical components at least as good as the best glass components have been fabricated for the ALS. Surface microroughness figures of $2 \AA$ rms-five times better than previously achievable-have been obtained on 15-inch-long copper alloy mirrors coated with electroless nickel. 
Currently we are working with Photon Sciences International to achieve this microroughness on metal surfaces that also have a slope error of only 0.5 $\mu \mathrm{rad} \mathrm{rms}$; the project is proceeding well. In addition, we developed, in collaboration with industry, a completely new method of making toroidal mirrors to a slope tolerance of $5 \mu \mathrm{rad} \mathrm{rms}$. These developments have opened a new era in the availability of high-quality grazing-incidence optical elements for the synchrotron radiation community. The significance of these multiyear, cooperative efforts, which benefit not only the ALS but our industrial partners and the synchrotron-radiation community in general, was recognized with the Federal Laboratory Consortium's Technology Transfer Award in 1992.

Both in construction and in the planning of the user program, we have placed great emphasis on health, safety, and environmental protection. Commissioning activities have been paced not only by the installation of the mechanical and electrical hardware, but also by installation of the safety systems deemed necessary for a particular activity. Commissioning activities are limited to the evenings because of ALS construction and installation activities during normal working hours. In late afternoon the linac and booster tunnels are searched and secured according to the established procedure. The accelerator enclosure is also locked, although measurements have shown that the radiation level outside the tunnels is well below the safety limit. Only then does operation begin.

Radiation safety is one of our major concerns. The linac and the booster generate high levels of radiation (neutrons and bremsstrahlung photons), confined inside the accelerator tunnels. According to calculations and actual radiation surveys made in normal and accident scenarios, the shielding performs as required in protecting the user area and points beyond from unacceptable radiation levels. In addition to the routine continuous monitoring, the radiation level in the accelerator building is surveyed every time commissioning reaches a milestone that is relevant to radiation.

Planning is currently under way for the Operational Readiness Review, a milestone that must be passed before the ALS can open as a user facility.

When the ALS opens its doors to a large and diverse community of users, the environment, health, and safety matters will become even more challenging. Accordingly, a major part of the user-services program focuses on experimental safety. Before coming to the ALS, users will fill out a form to describe their experiments, providing information about all potentially hazardous materials and equipment-for instance, flammable chemicals, lasers, or high-voltage power supplies. Eventually, the form will be accessible by computer so that users will have a convenient alternative for supplying this information. Whether entered on paper or by computer, the information will be stored in a computer database for easy retrieval. Safety specialists will review the forms and suggest ways to remedy any anticipated problems before an item is shipped to the ALS. This proactive review is intended to forestall the shipment of unacceptable equipment that would otherwise require modification before being installed.

A similar approach is planned to ensure beamline safety. Users who build beamlines are required to provide detailed documentation, including drawings, radiation and shielding calculations, interlock descriptions, and procedures, before bringing the beamline on site. These documents will be

\section{Environment, Safety, and Health}


evaluated by the Beamline Safety Committee, consisting of ALS staff members who have expertise in safety and in the design and construction of beamlines and other experimental systems.

Once at the ALS, users' beamlines, equipment, and experimental setups will be inspected by LBL and ALS safety personnel before being put into operation. A team of ALS operations coordinators will continue to oversee all activities on the ALS floor for compliance with safety regulations.

\section{Interaction with the User Community}

Close and extensive interaction with prospective users has been a hallmark of ALS program planning. The research program may be thought of in four categories:

- Materials, interface, and surface sciences.

- Atomic, molecular, and chemical sciences.

- Life sciences.

- Instrumentation and technology.

The intitial program emphasizes the high brightness of soft-x-ray and extremeultraviolet (XUV) light available from the ALS. The first experiments will be carried out by Participating Research Teams (PRTs)--groups of researchers from one or more institutions who have related interests. The primary responsibility for experimental apparatus rests with the PRTs; the responsibility for the beamlines and insertion devices will be shared by the ALS and the PRTs. In return for its commitment, each PRT receives a guaranteed fraction of the ALS operating time at its beamline. Through a proposal process, a substantial fraction of runining time at each beamline will also be made available to independent investigators not affiliated with a PRT.

Two complete beamlines, the front ends for three additional beamlines, and a diagnostic beamline are now under construction at the ALS. Extensive work is also under way to create a physical and procedural infrastructure that will help researchers use the ALS safely, ensily, and efficiently.

Beamlines 7.0, 8.0, and 9.0: Insertion-Device Beamlines
The two beamlines to be built entirely by the ALS are designated Beamlines 7.0 and 9.0. The numbers correspond with the arc sectors of the storage ring in which their radiation ports are located.

Beamline 7.0 will use as its light source the undulator U5.0, now nearly complete. This beamline will have a spherical-grating monochromator with three interchangeable diffraction gratings and will deliver photons over the energy range $70-1200 \mathrm{eV}$ to a spot as small as $50 \times 50 \mu \mathrm{m}$ (FWHM) at the sample. Present plans call for a photoelectron spectrometer at one end station, equipped with two microscopes-a scanning photoelectron microscope (SPEM) and a scanning transmission $x$-ray microscope (STXM)-both with better than $500-\AA$ resolution. Beamline 7.0 will be used for spectromicroscopy by a PRT whose spokesperson is Brian Tonner of the University of Wisconsin.

The source of light for Beamline 9.0 is the undulator U8.0, now being assembled. Equipped with an SGM that has three interchangeable gratings, the beamline will deliver photons in the $20-300 \mathrm{eV}$ range to a spot as small as $50 \times 80 \mu \mathrm{m}$ (FWHM) at the sample. Beamline 9.0 will be used to investigate photoprocesses in atoms, molecules, and ions. 
While its front end is under construction at the ALS, a third undulator beamline (designated 8.0) is being built at IBM's Aimaden Research Center in San Jose, California, by a PRT that includes members from IBM, Tulane University, and the University of Tennessee. This beamline's source is the second U5.0, also under construction at the ALS. The PRT, whose spokesperson is Joachim Stöhr, will use the beamline for photoelectron spectroscopy and soft X-ray emission (SXE) and absorption (SXA) spectroscopy.

Two bend-magnet beamlines, designated 9.3 .2 and 10.3, will also come on line in spring 1993. Beamline 9.3.2 formerly operated as Beamline 6-1 at the Stanford Synchrotron Radiation Laboratory. Its major components are being moved to the ALS and modified to make the best use of this facility's brighter synchrotron radiation. The beamline has an SGM with three interchangeable gratings, thus covering a broad range of photon energies-from $30 \mathrm{eV}$ to $1.5 \mathrm{keV}$. The spot size at the sample will measure about $100 \mu \mathrm{m}$ (vertical) $\times 500 \mu \mathrm{m}$ (horizontal). Beamline 9.3.2 features an experimental chamber outfitted with an angle-resolved photoemission spectrometer (ARPES) system. The beamline will be used to study problems in materials and chemical sciences by a PRT whose spokesperson is Charles S. Fadley of the University of California at Davis and of LBL.

Beamline 10.3 is being built by LBL's Center for X-Ray Optics (CXRO). It will include white-light microprobes-one existing (a past winner of the R\&D 100 award) and one of novel design to detect and determine trace quantities of elements as small as $10^{-15} \mathrm{~g}$. Its two branches employ mirrors to select light around $10 \mathrm{keV}$ and focus it to extremely small spot sizes. The existing microprobe on one branch will use spherical mirrors in a Kirkpatrick-Baez configuration to achieve a spot size of 3-4 $\mu \mathrm{m}^{2}$. The second branch will include a microprobe with "tiny bendable mirrors" -flat metal pieces bent into an elliptical shape-to achieve a spot size of less than $1 \mu \mathrm{m}^{2}$. The beamline will be operated by a PRT whose spokesperson is Albert Thompson of CXRO.

At least five additional beamlines are planned for the first few years of ALS operation. Two undulator beamlines would support, respectively, $x$-ray microscopy in the life sciences and projection x-ray lithography technology development. Three bend-magnet beamlines would be used for highresolution spectroscopy of solid-state and gas-phase systems and for x-rayoptics metrology. In addition, the Program Review Panel approved two new proposals for a chemical dynamics branchline on the U8.0 undulator beamline and for a bend-magnet $x$-ray microscopy life sciences beamline. Planning is also under way for an insertion device and beamline to provide circularly polarized VUV and soft $x$-ray radiation, which will be used for spectroscopy and spectromicroscopy of magnetic materials and other physical and biological systems.
Beamlines 9.3.2 and 10.3: Bend-Magnet Beamlines

Future Beamlines 


\section{Availability to Researchers}

In addition to PRTs, the ALS is open to qualified independent investigators who submit proposals for beamline access. Although largely free from the responsibility of furnishing experimental facilities, independent investigators may choose to provide their own experimental chambers. As a Department of Energy user facility, the ALS provides all qualified investigators with free beamtime.

Initially, three beamlines (the undulator beamlines 7.0 and 9.0 and the bend-magnet beamline 9.3.2) are expected to be available to independent investigators. A fourth beamline, with a bend-magnet source and a doublecrystal monochromator, will be available for experiments in 1995. Further details may be found in PUB3104, ALS Beamlines for Independent Investigators, which is available on request from the ALS. 
To make use of the ALS as convenient as possible, a comprehensive program to cover all aspects of a user's visit is being developed after considerable study of other user facilities. For example, an LBL Visitor Center near the shuttle-bus terminal will provide "one-stop shopping"; to wit, users can register with the Personnel Department, obtain an identification badge, take their basic safety training, and receive a parking permit, all at the same location.

Experimental and beamline safety will be a major part of the program at all stages. ALS and LBL Environment, Health and Safety Division staff will take a proactive approach to safety, reviewing operating plans and equipment designs well before the users and their apparatus arrive. This attention to safety will include pre-operations checking after the equipment has been set up and will continue with oversight during operations.

Users will be assisted throughout by operations coordinators under the supervision of a user support manager. These facilitators will help users take full advantage of the crafts and trades support available at LBL and will provide a wide range of logistics assistance. Extensive training and documentation will also aid experiment planning and help ensure safe, efficient operation.

An important planning role has been played by an ongoing series of workshops. The ALS workshops, along with related meetings sponsored by other organizations, have helped highlight the wide variety of scientific opportunities for high-brightness XUV light and further broaden the participation by all segments of the potential user community. They have also been useful in helping us understand the community's needs.

Synchrotron radiation has numerous current and potential applications in geochemistry, mineralogy, and other geological disciplines. In December 1991, in conjunction with the San Francisco meeting of the American Geophysical Union, a workshop was held on "Applications of the Advanced Light Source to Problems in the Earth, Soil, and Environmental Sciences." Topics included $x$-ray microprobe analysis, $x$-ray spectroscopy, and surveys of current applications.

To help the industrial research community to become better acquainted with the ALS, a workshop spotlighted some of the ways in which XUV radiation from synchrotron sources has already been put to use by industrial scientists.

More than 80 participants and speakers from 20 countries attended the NATO Advanced Study Institute workshop on "New Directions in Research with Third-Generation Soft X-Ray Synchrotron Radiation Sources," held June 28-July 10, 1992 in Maratea, Italy. This gathering was a milestone in international scientific programs sponsored by NATO: for the first time, representatives of former Eastern Bloc countries (Russia and Hungary) were not only allowed but encouraged by NATO to participate. The institute focused on research opportunities at third-generation synchrotron radiation facilities, including the ALS, Elettra (a machine of comparable performance being built in Trieste, Italy) and BESSY II in Berlin. Speakers described how the unprecedentedly bright light from these and similar sources can be used for research in materials science, surface science, atomic physics, chemistry, and biological science.
User Services

Workshops and ALS Science 
"Synchrotron Radiation in Transactinium Research" was the subject of a workshop held October 1-2, 1992 at LBL. The workshop explored the scientific opportunities that would be offered by an actinide beamline at the ALS. The proposed beamline would deliver synchrotron radiation in the energy range $20-1000 \mathrm{eV}$ for the study of the transactinium elements and their compounds.

The major question in studies of the physical and chemical properties of these elements is the role of the $5 f$ electrons. The ALS offers the high brightness and spectral range necessary to study the electronic properties of the actinides both in the vapor phase and in the solid state. Researchers will be able to probe the $5 f, 6 d, 7 s$, and inner electron shells unambiguously, in addition to exploring the unique role of the $5 f$ electrons throughout the actinide series. Data from such studies, combined with theoretical calculations, would result in a coherent picture of $5 f$ electron systems.

\section{ALS Adivisory Panels}

Two committees provide advice on ALS planning and operation to the LBL Director. The Science Policy Board (see Table 3-1) provides advice on highlevel policy issues affecting the ALS. The Program Review Panel (Table 3-2) gives advice on the scientific program through the ALS Director. Until the ALS is commissioned, this panel's main task will be evaluation of PRT proposals and reviews of progress by previously approved PRTs. Accordingly the panel devoted its February and August 1992 meetings to proposals

Table 3-1. ALS Science Policy Board, 1992

Dean E. Eastman, IBM Thomas J. Watson Research Center (chair)

E. Morton Bradbury, School of Medicine, University of California at Davis William F. Brinkman, AT\&T Bell Laboratories

John C. Browne, Los Alamos National Lo'soratory

Bernd Crasemann, University of Oregon

J. McEwan Paterson, Stanford Linear Accelerator Center

Table 3-2. ALS Program Review Parel, 1992

Neville V. Smith, AT\&T Bell Laboratories (chair)

Sheldon Datz, Oak Ridge National Laboratory*

Charles S. Farley, University of California at Davis and $\mathrm{LBL}^{+}$

John W. Hepburn, University of Waterloo, Ontario, Canada

Franz J. Himpsel, IBM Thomas J. Watson Research Center

Michael L. Knotek, Battelle Pacific Northwest Laboratories*

Christof Kunz, University of Hamburg ${ }^{\dagger}$

Gerald J. Lapeyre, Montana State University

Giorgio Margaritondo, École Polytechnique Féderalé (Lausanne, Switzerland)

Keith Moffat, University of Chicago*

William Orme-Johnson, Massachusetts Institute of Technology

François Wuilleumier, University of Paris-South ${ }^{\dagger}$

\footnotetext{
* Retired from committee in 1992.
}

${ }^{+}$New member for 1992. 
from both insertion-device and bend-magnet teams (three were approved) and to discussing guidelines for PRT progress. The panel also encouraged proposals from independent users once the ALS becomes operational.

The ALS Users' Association held its fifth annual meeting in August 1992 at LBL. The meeting highlighted scientific opportunities (many of them based upon ongoing work elsewhere) that are likely to be explored during the first few years of ALS operation. Other highlights included the Department of Energy's view of funding prospects for experimental facilities (insertion devices, beamlines, and end stations).

At the meeting, new members were nominated to the Users' Executive Committee, which serves as the voice of the user community in communicating its needs to the ALS staff. The UEC is an elected body comprising 11 members with staggered terms of three years. Table 3-3 lists the 1992 memb.rs. During 1992, the UEC made important contributions to planning for ALS operations, defining space requirements for user offices and laboratories, and devising the user safety program.

Table 3-3. ALS Users' Executive Committee, 1992

C. Denise Caldwell, University of Central Florida

George Castro, IBM Almaden Research Center

Stephen P. Cramer, University of California at Davis

David L. Ederer, Tulane University (vice chair)

Cynthia Friend, Harvard University

Dennis W. Lindle, University of Nevada at Las Vegas

Rupert C. Perera, Lawrence Berkeley Laboratory

Piero A. Pianetta, Stanford Synchrotron Radiation Laboratory (chair)

James G. Tobin, Lawrence Livermore National Laboratory

Brian P. Tonner, University of Wisconsin at Milwaukee and Synchrotron

Radiation Center, University of Wisconsin at Madison

Michael G. White, Brookhaven National Laboratory
ALS Users' Association 


\section{Publications and Presentations}

J.M. Bizau, D. Cubaynes, M. Richter, F.J. Wuilleumier, J. Obert, J.C. Puteaux, T.J. Morgan, S. Sorenson, E. Källne, A. Damany, and A.S. Schlachter, "First measurement of a photoelectron spectrum emitted in the photoionization of a singly charged ion beam by synchrotron radiation," Seventeen International Conference on Electron and Atom Collisions (Brisbane, Australia, 1991).

F. Cerina, C. Capasso, S. Liang, W. Ng, A.K. Ray-Chaudhuri, G. Margaritondo, J.H. Underwood, J.B. Kortright, and R.C.C. Perera, "First results from a soft $x$-ray photoemission microscope MAXIMUM with $1000 \AA$ spatial resolution," Tenth International Conference on Vacuum Ultraviolet Radiation Physics (Paris, France, 1992).

M.W. Clark, J.A. Tanis, E.M. Bernstein, N.R. Badnell, R.D. DuBois, W.G. Graham, T.J. Morgan, V.L. Plano, A.S. Schlachter, and M.P. Stockl, "Cross sections for resonant transfer and excitation in $\mathrm{Fe}^{8+}+\mathrm{H}_{2}$ collisions," Phys. Rev. A 45, 7846 (1992).

C. Cork and H. Nishimura, "Framework for control system development," International Conference on Accelerator and Large Experimental Physics Control Systems (Tsukuba, Japan, 1991); LBL-31580 (1991).

D. Cubaynes, J.M. Bizau, C. Marinelli, T.J. Morgan, J. Novak, M. Pahler, B. Rouvellou, F. Schlachter, N. Berrah Mansour, and F.J. Wuilleumier, "First measurements of the asymmetry parameter $b$ for photoelectrons emitted from the $2 \mathrm{p}$ subshell of $\mathrm{Na}$ atoms in ground and 3p-laser excited states," Tenth International Conference on Vacuum Ultraviolet Radiation Physics (Paris, France, 1992).

D. Cubaynes, J.M. Bizau, T.J. Morgan, J. Novak, M. Pahler, B. Rouvellou, F. Schlachter, and F.J. Wuilleumier, "Angular distribution in the direct photoionization of the $2 p$ subshell in ground and excited state Na atoms," Conference on Atomic Physics (Riga, Latvia, 1992).

W.V. Hassenzahl, "Progress of the ALS," "Status of ALS insertion device design," "Magnetic structure of ALS insertion devices," "Magnetic field measurements of insertion devices," and "Superconducting magnetic energy storage," Beijing Synchrotron Radiation Facility (Beijing, People's Republic of China, 1991).
W.V. Hassenzahl and D. Phelan, "Tests of a model pole assembly for the ALS U8.0 undulator," LBL-31960) (1992).

P.A. Heimann, E.M. Gullikson, and W.R. McKinney, "The efficiency of a blazed and a laminar grating," Tenth International Conference on Vacuum Ultraviolet Radiation Physics (Paris, France, 1992).

P. Heimann, T. Warwick, M. Howells, W. McKinney, D. DiGennaro, B. Gee, D. Yee, and $B$. Kincaid, "The Advanced Light Source U8 beam line, 20-300 eV," in Proceedings of the Seventh National Conference on Synchrotron Radiation Instrumentation (Baton Rouge, LA, 1991), Nucl. Instrum. Meth. A 319, 106 (1992); LBL-32155 (1991).

J. Hinkson, "Advanced Light Source beam position monitor," Accelerator Instrumentation Workshop at CEBAF (Newport News, VA, 1991); LBL-31526 (1991).

M. Howells, "Optical systems for synchrtotron radiation beamlines," NATO Advanced Study Institute on New Directions in Research With Third-Generation Soft $X$ Ray Synchrotron Radiation Sources (Maratea, Italy, 1992).

M. Howells and P. Heimann, "Optical systems for synchrotron radiation beamlines," U.S. Particle Accelerator School (Austin, TX, 1992).

E. Hoyer, "High power photon beamline elements in the LBL/SSRL/Exxon Beamline VI," LBL-32981 (1992).

S.C. Irick, "Determining surface profile from sequential interference patterns from a long trace profiler," in Proceedings of the 4th International Conference on Synchrotron Radiation Instrumentation (Chester, UK, 1991), Rev. Sci. Instrum. 63, 1432 (1992).

S.C. Irick, W.R. McKinney, D.L.J. Lunt, and P.Z. Takacs, "Using a straightness reference in obtaining more accurate surface profiles from a long trace profiler," in Proceedings of the 4th International Conference on Synchrotron Radiation Instrumentation (Chester, UK, 1991), Rev. Sci. Instrum. 63, 1436 (1992).

S.C. Irick and W.R. McKinney,

"Advancements in one-dimensional profiling 
with a long trace profiler," International Symposium on Optical Fabrication, Testing, and Surface Evaluation Conference (Tokyo, Japan, 1992); LBL-32560 (1992).

B.M. Kincaid, "The use of undulator radiation in VUV and soft $x$-ray radiometry," Optical Society of America Meeting (San Jose, CA, 1991); LBL-31584 (1991).

B. Kincaid, "Undulators in the real world," NATO Advanced Study Institute on New Directions in Research With ThirdGeneration Soft X-Ray Synchrotron Radiation Sources (Maratea, Italy, 1992).

B. Kincaid, "Undulators: the state of the art," 10th International Conference on Vacuum Ultraviolet Radiation Physics (Paris, France, 1992).

S. Magyary, M. Chin, M. Fahmie, $H$. Lancaster, P. Molinari, and A. Robb, "Status report on the Advanced Light Source control system," International Conference on Accelerator \& Large Experimental Physics Control Systems (Tsukuba, Japan, 1991); LBL-31436 (1991).

W.R. McKinney, S.C. Irick, and D.L.J. Lunt, "Water cooled metal optics for the Advanced Light Source," in Procedings of the Seventh National Conference on Synchrotron Radiation Instrumentation (Baton Rouge, LA, 1991), Nucl. Instrum. Meth. A 319, 179 (1992); LBL-31259 (1991).

R.C.C. Perera, "Photon excitation for satellite free $x$-ray spectroscopy: instrumentation challenges," in Procectings of the Seventh National Conference on Synchrotron Radiation Instrumentation (Baton Rouge, LA, 1991), Nucl. Instrum. Meth. A 319, 277 (1992); ; L.BL-31741 (1991).

R.C.C. Perera, "Synchrotron radiation source physics," "ALS diagnostic beamline," and "Overview of the ALS storage ring," 6th Chinese International Summer School of Physics on Applications of Synchrotron Radiation (Beijing, People's Republic of China, 1992).

R.C.C. Perera, M.E. Melczer, A. Warwick, A. Jackson, and B.M. Kincaid, "Diagnostic beam line for a third generation storage ring," in Procedings of the 4 th International Conference on Synchrotron Radiation Instrumentation (Chester, UK, 1991), Rev. Sci. Instrum. 63, 541 (1992).
T. Reich, Z. Hussain, E. Moler, G. Kaindl, D.A. Shirley, and M. Howells, "High resolution in the soft $x$-ray range with a toroidal grating monochromator," 10 th International Conference on Vacuum Ultraviolet Radiation Physics (Paris, France, 1992).

A.L. Robinson and A.S. Schlachter, "Scientific program of the Advanced Light Source at LBL," in Procedings of the Seventh National Conference on Synchrotron Radiation Instrumentation (Baton Rouge, LA, 1991), Nucl. Instrum. Meth. A 319, 40 (1992); LBL-31564 (1991).

A.L. Robinson, "The Advanced Light Source at Lawrence Berkeley Laboratory,", in Proceedings of the 4th International Conference on Synchrotron Radiation Instrumentation (Chester, UK, 1991), Rev. Sci. Instrum. 63, 1555 (1992); LBL-31243 (1992).

R. Savoy and W.V. Hassenzahl, "Periodic magnetic structure design calculations for the U8.0 undulator," LBL-32025 (1992).

L. Schachinger, "Summary of the Working Group on Modeling and Simulation," Fifth Advanced ICFA Beam Dynamics Workshop (Corpus Christi, TX, 1991); LBL-31665 (1991).

A. Schlachter, "Introduction to thirdgeneration synchrotron radiation sources," NATO Advanced Study Institute on New Directions in Research With ThirdGeneration Soft X-Ray Synchrotron Radiation Sources (Maratea, Italy, 1992).

R.D. Schlueter, "Harmonics suppression in electromagnets with application to the ALS storage ring corrector magnet design," LBL32028 (1992).

R.D. Schleuter, "Harmonics suppression of vacuum chamber eddy current induced fields with application to the Superconducting Super Collider (SSC) Low Energy Booster (LEB) magnets," LBL-32026 (1991).

R.D. Schlueter, "Iron yoke eddy current induced losses with application to the ALS septum magnets," LBL-32027 (1992).

D.H. Schneider, D.R. DeWitt, R.W. Bauer, J.R. Mowat, W.G. Grah;am, A.S. Schlachter, B. Skogvall, P. Fainstein, and R.D. Rivarola, "Absolute doubly differential cross sections for electron emission in collisions of 3.5$\mathrm{MeV} / \mathrm{u} \mathrm{Fe}^{17+}$ and $\mathrm{Fe}^{22+}$ ions with $\mathrm{He}$ and $\mathrm{Ar}$ 
gas targets," in Proceedings of the 10th International Conference on Vacuum Ultraviolet Radiation Physics (Paris, France, 1992), Phys. Rev. A 46, 1296 (1992).

D. Shu, T. Warwick, and E.D. Johnson, "Diagnostic phosphors for the Advanced Light Source," in Proceedings of the 4th International Conference on Synchrotron Radiation Instrumentation (Chester, UK, 1991), Rev. Sci. Instrum. 63, 548 (1992).

J.A. Tanis, R.D. DuBois, and A.S. Schlachter, "Comment on 'double and single ionization of helium by high-velocity $\mathrm{N}^{7+}$ ions'," Phys. Rev. Lett. 68, 897 (1992).

T. Warwick and P. Heimann, "Novel characteristics of VUV insertion device beamlines at the Advanced Light Source," in Proceedings of the Seventh National
Conference on Synchrotron Radiation Instrumentation (Baton Rouge, LA, 1991), Nucl. Instrum. Meth. A 319, 77 (1992); ; LBL-31533 (1991).

T. Warwick and S. Sharma, "Thermal effects and mirror surface figure requirements for a diagnostic beamline at the Advanced Light Source," in Proceedings of the Seventh National Conference on Synchrotron Radiation Instrumentation (Baton Rouge, LA, 1991), Nucl. Instrum. Meth. A 319, 185 (1992); ; LBL-31532 (1991).

T. Warwick, D. Shu, B. Rodricks, and E.D. Johnson, "Prototype photon position monitors for undulator beams at the Advanced Light Source," in Proceedings of the 4th International Conference on Synchrotron Radiation Instrumentation (Chester, UK, 1991), Rev. Sci. Instrum. 63, 550 (1992). 


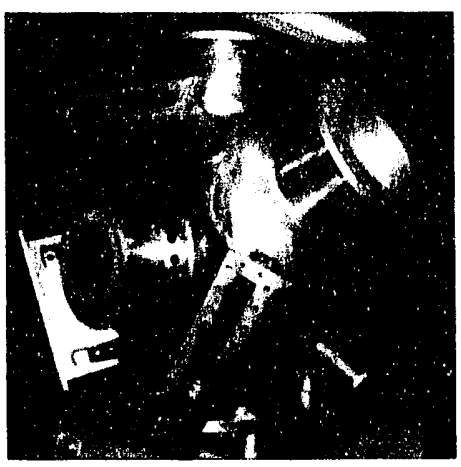

4.

\section{CENTER FOR BEAM PHYSICS}

T

WO MAJOR ACCELERATOR-BASED INITIATIVES are being assisted by the Center for Beam Physics, ${ }^{*}$ a divisional center that performs multifaceted exploratory studies of the physics of accelerators and beams. PEP-II, a proposed B-meson "factory" based on the Positron-Electron Project ring at the Stanford Linear Accelerator Center, has been the subject of ongoing research. Meanwhile, a long-standing interest in free-electron lasers and highbrightness electron and photon sources has led to the detailed design of an infrared free-electron laser (IRFEL), which is proposed as part of the Chemical Dynamics Research Laboratory. The IRFEL investigations have led to productive collaborations with Stanford University, Brookhaven National Laboratory, the Continuous Electron Beam Accelerator Facility, and TRW, Inc.

S. Chattopadhyay (heat)

K.-J. Kim (ilepulty)

High-Luminosity Collider Physics

M. Zisman (grolup leader)

Y.-H. Chin

E. Forest

M. Furman

A. Garren

R. Rimmer

D. Robin

A. Zhulents

High-Energy Collider Physics

A. Sessler (yromiplinder)

G. Fiorentini

R. Govil

S. Krishnagopal

W. Leemans

C. Rangarajan

D. Whittum

C. Wang

Radiation Sources and FELs

K.-J. Kim Cornup linterr

Y.-H. Chin
J. Edighoffer
R. Gough
K. LaMon
W. Leemans
S. Lidia
J. Smithwick
M. Xie
Storage-Ring Synchrotron Light
Sources and ALS Accelerator Systems
A. Jackson (group lander)
J. Bengtssun
R. Keller
C. Kim
D. Massoletti
H. Nishimura
L. Schachinger
F. Selph
Beam Electrodynamics
J. Corlett (grotul leader'
W. Barry*
J. Byrd

D. Goldberg

W. Harnden*

J. Johnson ${ }^{*}$

C. Lambertson

R. Rimmer

1. Smithwick*

F. Voelker*

J. Wise*

International Visitors

G. Fiorentini (Univ. of Milan)

M. Meddahi (CERN)

D. Whittum (KEK)

C. Wang (P'RC)

Students

G. Fiorentini

R. Govil

K. LaMon

S. Lidia

E. Wallace

Principal Collaborators

W. Barletta (LI.NL)
1. Ben-Zvi (BNL)

J. Dorfan (SLAC)

F. Dylla (CEBAF)

S. Fornaca (TRW)

D. Hitlin (Caltech)

C. Leeman (CEBAF)

P. Oddone (1.BL)

A. Schwettman (Stanford)

C. Shank (LBL)

G. Westenskow (LLNL)

Technical Support

D. Massoletti

J. Smithwick*

J. Wise

Administrative Support

J. Kono (center admimistrator)

E. Atkins-Orvis

M. Condon

M. Mara-Ann

D. Moretti

* Engineering Division

- The Center was formerly known as the Exploratory Studies Group. 
To meet the technical challenges of these initiatives and to generally enhance LBL's capabilities in particle- and photon-beam research, the Center made several additions to its experimental capability in 1992 and early 1993. In addition to upgrading the beam electrodynamics laboratory and setting up an optics lab, the detailed design of the Advanced Test Beam Facility was finished and construction was started. This facility will get "double the money's worth" out of the 50-MeV ALS linac by using it for beam physics experiments during the considerable spans of time between the ALS injection cycles. Many experiments are possible; immediate plans include generation and detection of $x$-ray pulses as short as tens of femtoseconds (a long-standing interest) and focusing of a beam by using plasma lenses.

Members of the Center have been involved in the ALS project from the outset and make up its technical core, the Accelerator Systems Group. With the design of the accelerators complete, they have continued to play major roles in guiding construction, programming the control system, and commissioning.

Research continues in accelerator theory, nonlinear dynamics, and fundamental FEL physics. The High-Energy Collider Physics group continued its long-range Two-Beam Accelerator research. The Beam Electrodynamics group contributed to and supervised the PEP-Il rf and feedback design efforts. It also contributed significantly to rf, impedance, and feedback work at the ALS, and worked on beam-cooling improvements for the Tevatron's antiproton source.

The worldwide hightenergy physics community has become increasingly interested in "B factories," which would produce $\mathrm{B} \overline{\mathrm{B}}$ pairs" for fundamental studies of chargeparity $(C P)$ violation and rare $B$-meson decays. Several schemes for copious $B \bar{B}$ production in electron-positron collisions have been advanced in the literature. In collaboration with the Stanfort Linear Accelerator Center (SLAC), Lazerence Livermore National Laboratory ( $L L N L$ ), and Caltech, the Center is designing a facility bused on one of the most promising schemes: PEP-II, a collider with one high-energy ring and one lowe-energy stornge ring. This energy-asymmetric collider, built in the PEP tumnel at SLAC and re-using many PEP components, would be scientifically and economically atractive.

During 1992, the PEP-II collaboration continued refining the design of a B factory in which a 9-GeV electron beam in PEP collides with a $3.1-\mathrm{GeV}$ positron beam circulating in a new storage ring. The new low-energy ring will be of the same circumference as PEP and will be mounted above it in the existing tunnel, as shown in Figure 4-1. The chosen energy combination reaches the $\mathrm{E}(4 \mathrm{~S})$ resonance, at which $\mathrm{B} \overline{\mathrm{B}}$ pairs are produced in the abundance required for the study of $C P$ violation (sidebar). The challenge in the design of a B factory is to reach an initial luminosity of $3 \times 10^{33} \mathrm{~cm}^{-2} \mathrm{~s}^{-1}$, which is more than an order of magnitude beyond the luminosities achieved to date in electron-positron colliders.

\section{B-Factory Studies}

\author{
Conceptual Design \\ Overview
}

\footnotetext{
"A B meson and its antimatter equivalent produced together. Pronounced "bee bee-bar."
} 
Figure 4-1. The proposed asymmetric B factory, PEP-II, would be built in the Positron-Electron Project tunnel and would use a substantial amount of the existing hardware for the PEP collider. Recent LBL work in the multi-institutional PEP-II collaboration has emphasized refinement of the magnetic-lattice designs of the two rings and extensive studies of beam-beam interaction. (Artist's impression courtesy SLAC)

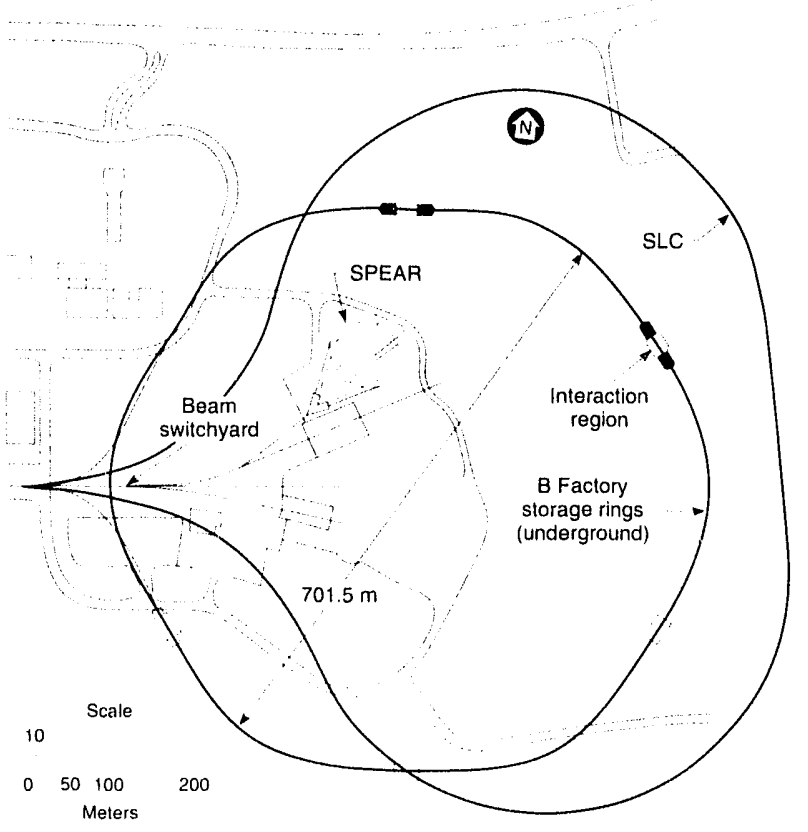

XBL 902-5762

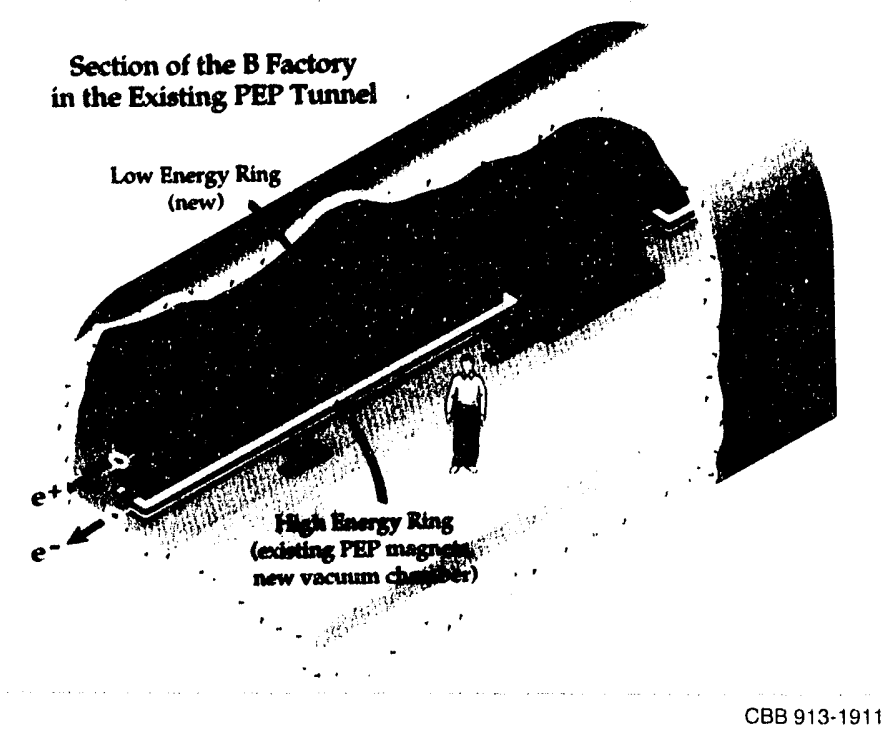

In principle, all the relevant parameters-the ratio of the cross sections of the two beams, the beam currents, the beam-beam tune shifts, the beam energies, and the vertical beta functions at the interaction point-are adjustable. In practice, however, the beambeam tune shift cannot be increased beyond a certain value, which has been determined experimentally in many colliders to lie in the range of 0.02 to 0.06 . Simi-

larly, a collision energy at the $\longleftarrow(4 S)$ resonance implies that the product of the beam energies must be $28 \mathrm{GeV}^{2}$. Thus, only three parameters-the beam-size ratio, the beam current, and the vertical beta function at the interaction point-are fully at the discretion of the accelerator designer.

The chosen luminosity, $3 \times 10^{33} \mathrm{~cm}^{-2} \mathrm{~s}^{-1}$, has been shown to be adequate for the study of the key physics issue, $\mathrm{CP}$ violation. Given the limitations caused by the beam-beam interaction-which we take for our design to correspond to a maximum tune shift of 0.03 - a substantial increase in luminosity implies that the high-current beam must be divided into a large number of individual bunches (1658 in our design). This approach involves a design in which the single-bunch parameters (emittance, bunch length, current, tune shift, etc.) are well within present practice for colliders. Our choice of 1658 bunches lies in a safe middle ground between the extremes-it does not exacerbate coupled-bunch instabilities, nor does it have problems with single-bunch effects. 
For PEP to serve as the high-energy ring, several of its systems must be significantly upgraded to deal with these issues. Foremost among these are the $\mathrm{rf}$ and vacuum systems. The high luminosity leads to extreme heat loads caused by synchrotron radiation; a great deal of engineering work has been performed by our collaborators to solve the resultant cooling and vacuum problems. There are also implications for the rf system of the high-energy ring. It will operate at a frequency of $476 \mathrm{MHz}$ to phase-lock the storage ring $\mathrm{rf}$ system to that of the 2856- $\mathrm{MHz}$ injector (the "two-mile linac" that is also used to inject the Stanford Linear Collider). This choice of frequency minimizes injection phase errors, which contribute significantly to the power demands of the multibunch feedback system. The rf system will consist of 20 single-cell cavities; the cavitv design itself is aimed at minimizing the higherorder-mode impedance contribution of the rf system.

The interaction region is designed in such a way that the beams collide head on. During 1992, a great deal of effort has gone into studying the beam-beam interaction and into refining the lattice designs of the two rings and the interaction region. These three subjects are closely related.

\section{B Decays and CPT Symmetry}

Judging particle interactions by the standards of the familiar, macroscopic world, one would think that if a process and the participating objects were replaced either by their antimatter equivalents or by versions of themselves as seen in a mirror, the rate of the process would remain the same. It seems equally intuitive that reversing a process would yield the original participants, much as though one were running a movie in reverse and watching the actors run backward in their own footprints.

But on the scale of subatomic particles and the quarks that make them up-a scale where the "weak interaction" becomes the strongest of forces-the first two rules, called "conservation of parity" and "charge conjugation," are not necessarily obeyed. Not even CP symmetry, which combines both rules, necessarily holds true. The remaining variable is time; we are left with CPT symmetry-a scheme in which C, P, and CP symmetry violations can occur, but only if the arrow of time is allowed to take a different course when reversed, going back to a different beginning.

Thus far, $\mathrm{CP}$ violation has been observed through asymmetries in the decay modes of the neutral $\mathrm{K}$ meson and its antiparticle. The $\mathrm{K}^{0}$ and $\bar{K}^{0}$ contain an unusual quark, the "strange" quark, which is not found in the group of quarks that make up ordinary matter. The $\mathrm{K}$ decays in a wide variety of fashions (it is axiomatic that every decay mode that is not explicitly forbidden must occur eventually). In a few of these modes, the $\mathrm{K}^{0}$ decays a few tenths of a percent differently than the $\mathrm{K}^{0}$, a sign of $\mathrm{CP}$ violation. But studies of the $\mathrm{K}$ system have left many questions unanswered about the mechanisms and magnitude of $\mathrm{CP}$ violation.

The B meson, which contains a different unusual quark ("bottom" as opposed to "strange"), is predicted by the Standard Model of Particles and Interactions to have asymmetries as great as 30\% in some rare decay mndes. This makes it a very promising candidate for $\mathrm{CP}$-violation studies. However, the branching fraction-the proportion of pairs that will not only decay through the unusual modes but also violate $C P$ symmetry in doing so-is only about $10^{-4}$ to $10^{-5}$. Therefore, about $10^{7}$ to $10^{8} \mathrm{BB}$ pairs will have to be produced to get good $\mathrm{CP}$-violation statistics. This requirement, implying the need for many $\mathrm{e}^{+} \mathrm{e}^{-}$collisions, brings us to the luminosity frontier of accelerator physics, whose technical challenges are described elsewhere in this chapter.

The ultimate goal of this research is to enhance the Standard Model-today's partial theory of the building blocks of nature and how they interact-or replace it with a new, more-satisfactory theory. In either case, $\mathrm{CP}$ violation will have to be better quantified, and its origins will have to be explained. The present Standard Model does not disallow CP violation but does not explain it either. These studies also have ramifications beyond particle physics.

In 1967, not long after the discovery of $\mathrm{CP}$ violation, Andrei Sakharov pointed out that it might explain one of the long-standing riddles of cosmology: why the universe was not born with equal, evenly distributed quantities of matter and antimatter that would annihilate each other whenever they interacted. For some reason, the laws of nature appear to prefer matter over antimatter-a phenomenon that makes possible the physical universe we see every day. Such will be the implications of the research at PEP-II. 


\section{Lattice Design}

\section{Beam-Beam Interaction Studies}

The basic lattice designs of both rings were completed in earlier years; the primary effort during 1992 was, and remains, optimization of the lattices (particularly that of the low-energy ring). Using simulation tools that we had developed to analyze the dynamic behavior of particles circulating in storage rings, we found that the low-energy ring was not as dynamically stable as the high-energy ring. These tools are based on single-particle tracking, and fully account for nonlinearities, magnetic-field imperfections, and magnet-position errors. (Beam-beam interaction is ignored at this stage of the design.)

Significant strides have been made toward understanding the problem. Good dynamic behavior requires a stable aperture at least 10 times the natural beam size. However, one cannot make a positron beam that is of the same quality as the electron beam; its natural emittance and energy spread are each roughly 1.5 times larger. Therefore, all else being equal, one must achieve a larger aperture in the low-energy ring than in the high-energy ring. In addition, the stronger focusing of the low-energy ring requires stronger sextupole magnets in order to correct the linear chromaticity. As a result of this correction, the low-energy ring has more nonlinear chromaticity, as well as a greater tune shift under amplitude variations, than the high-energy ring. All this reduces the available dynamic aperture.

Our present results show that, to control these effects, it is necessary to have a local chromaticity-compensation scheme in both planes near the interaction point. Such a scheme requires sextupole magnets in the interaction-region straight section. They must be placed in such a way as to correct the chromaticity arising from the interaction-region quadrupole magnets without generating higher-order tune shifts. Our simulations have shown this scheme to be the best method of improving the dynamic aperture. As we approach a final design for the interaction region, the challenge we face is to implement this scheme while respecting the geometrical constraints imposed by the existing tunnel and by the relative position of the two rings.

While working on the lattice design, we also intensified our studies of beambeam effects. We have made a great deal of progress on a simulation approach that complements the lattice studies by providing detailed simulation of beambeam dynamics but neglecting magnet nonlinearities. These beam-beam studies are based on multiparticle tracking simulations, so they require much more computation than the single-particle dynamic-aperture studies. Ideally we would like to combine the two approaches into one consistent whole; we intend to develop the necessary tools for this unification during 1993.

Most of these studies were based on multiparticle simulations in the linear-lattice approximation. In the ongoing detailed-design effort, a good deal of additional progress has been made on various issues, including the existence of an adequate "working point" in the multivariate parameter space, adequacy of the beam separation at the parasitic collision points, beam-beam interaction during injection, departures from full equality of the four beam-beam parameters, and an experimental proposal at TRISTAN ${ }^{*}$ to test the effects of the beam-beam force on the closed orbit.

In search of a working point, we performed tune scans over a wider region of the tune plane than we had in the initial effort that produced the 1991 Conceptual Design Report. A working point above the half-integer, with

\footnotetext{
- Transposable Ring Intersecting STorage Accelerators in Nippon, a collider in Japan.
} 
fractional tunes $\left(v_{x}, v_{y}\right)=(0.64,0.57)$ for both beams, was shown to give adequate luminosity and has been adopted. The "old" working point, $\left(v_{x}, v_{y}\right)=(0.09,0.05)$, also gave acceptable beam-beam dynamics, but it was deemed to be too close to the integer, where we would expect to encounter serious closed-orbit instabilities. Ongoing simulations suggest that making the working points slightly different for the two beams will give even better performance.

Because of the relatively close bunch spacing, the bunches experience glancing collisions in the horizontal plane on their way into and out of the interaction point; we call these "parasitic collisions" (PCs). Although each beam experiences several of these collisions, the PC closest to the interaction point is the most disruptive. In the earlier interaction-region design, the separation of the two beams at the first PC was specified to be $2.8 \mathrm{~mm}$. Early simulations showed that if this distance were too small, the PCs could be quite detrimental to luminosity. Although the simulations proved that 2.8 $\mathrm{mm}$ was adequate, they also showed that if the distance were slightly smaller, the beam blowup could become substantial. For this reason the design of the interaction region has been upgraded to provide a much more comfortable 3.5-mm separation.

When the beams are injected, they are displaced transversely from their nominal orbits by a distance of approximately eight times their diameter. Therefore, if the injection were in the horizontal plane, the beams could collide head on, or almost head on, at the PC points. Simulations showed that, indeed, beam-beam-induced beam blowup was substantial during the first few damping times. When a vertical injection option was simulated, the beam-beam effect was found (as we had expected) to be much less severe, so the design now calls for vertical injection.

The design for PEP-II, which is asymmetric in energy, currently uses the same values in both rings for each of the four beam-beam tune shift parameters because this causes the beam-beam dynamics to resemble those of symmetrical colliders, which are well understood. Furthermore, this equality has the practical advantage of constraining many other parameters. Recent simulations suggest, however, that if the beam-beam parameters were somewhat different from each other, luminosity performance could be improved. We have explored two approaches thus far. In the first, the horizontal and vertical beam-beam parameters are kept equal to each other, but are different in the two beams. In the second, the horizontal and vertical beam-beam parameters are different from each other, but are kept equal for the two beams.

Simulations for both cases suggest that better performance can be obtained in the first case by using a slightly smaller beam-beam parameter for the positron beam and in the second case by using a slightly smaller vertical beambeam parameter for both beams. The possible effects of these choices upon other design parameters have been identified but not yet quantitatively evaluated. Undoubtedly some combination of the two approaches, or a third approach in which all four beam-beam parameters are different, will prove to be optimal, so our studies continue. Already we know from these simulations that the beam-beam dynamics remain well behaved when the four parameters are not equal, indicating that, at least, our initial requirement for equality in these parameters has some room for adjustment.

Because the beams travel in different vacuum chambers in an asymmetric collider, the beam orbits near the interaction point need to be controlled to a much greater degree than in symmetric colliders to ensure head-on colli- 
sions. A feedback scheme used in the Stanford Linear Collider, based on the observation of the beam-beam-induced orbit deflection, has been extended to the PEP-II design and shown to be promising. The basic idea is to measure the beam-beam-induced distortion of the closed orbits of the two beams at many points well away from the interaction point. These measurements can guide the operation of a feedback "lever" to keep the beams in collision. An experiment to test this idea at TRISTAN is expected soon.

In summary, most of the challenges of the PEP-II design have been addressed satisfactorily, and technical solutions are in hand. We are continuing the detailed engineering design and fabrication of various components for testing purposes. Some areas of the conceptual design, which was submitted to the Department of Energy in February 1991, have been refined during 1992. Further suggestions for improvements continue to be put forth as the accelerator physics and other technical issues becorne better understood.

\section{Chemical Dynamics Research Laboratory}

\section{Research Prospects}

The CDRL, with its advanced lasers, will complement the ALS, which upon completion will be the world's brightest source of soft $x$-rays for basic and applied research. Collaborative CDRL researchers from industry, universities, and national laboratories will use the unique features of ALS x-ray beams-high spectral brightness and very short pulse length (nominally tens of picoseconds). Undulators in the storage ring will provide somewhat spatially coherent radiationsometimes referred to as "laserlike" - that is broadly tunable across the soft-x-ray to ultraviolet regions of the electromagnetic spectrum.

The CDRL experimental systems will be used for dynamic, spectroscopic, and structural studies of highly reactive molecules. Many of these are created during the early stages of combustion. These studies will take place in an experimental hall where light from the IRFEL or infrared chemical lasers, the ALS, and advanced conventional lasers at various wavelengths (Figure 4-3) can all be directed into experiment stations to study the dynamics of fastmoving chemical processes in detail. Such fundamental knowledge-which is beyond the reach of existing experimental facilities-is crucial for improving the efficiency of combustion and controlling the formation of pollutants, among other issues.

Scientists from other LBL divisions, the University of California at Berkeley, and Sandia National Laboratory-Livermore are developing the CDRL research program. Research results will feed directly into U.S. industries concerned with cleaner combustion, alternative fuel supplies, reduced pollution, ard improved industrial processes. This is important not only to industry but also to government, since many regions of the nation face significant economic curtailment during the coming decades if air pollution from mobile and stationary sources is not controlled more effectively. The CDRL can provide a foundation of fundamental understanding that will enable long-term success in solving these problems.
The Chemical Dynamics Research Laboratory (CDRL) is a key nerv facility in the multilaboratory Combustion Dynamics Initiative, which is being put forward by the DOE's Division of Chemical Sciences. The research proposed for the laboratory (sidebar) is iderstanding of chemical reactions.

The CDRL is proposed for $L B L$, with significant participation by Sandia National Laboratories, two institutions with complementary strengths. Sandia researchers will be responsible for the construction and operation of some of the experimental equipment and zoill play key roles in the scientific program and the transfer of combustion technology to industry. The Center's role in this national user facility is twofold. The Advanced Light Source will supply the CDRL with VUV and soft-x-ray light from two beamlines. Experiments at the $C D R L$ could also be greatly enhanced by a proposed infrared free-electron laser (IRFEL), which personnel from the Center are designing as a second-phase addition to the facility. Researchers from other $L B L$ divisions, most notably the Chemical Sciences Division, will join colleagues from the University of California at Berkeley and other institutions in conduct. ing the scientific program. 

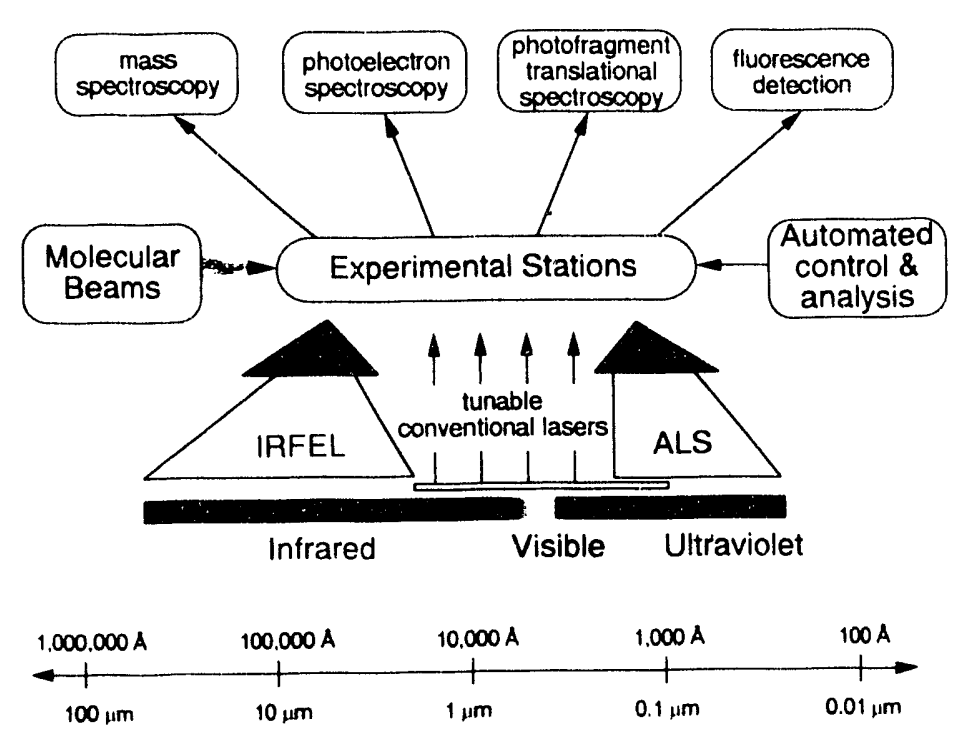

Electromagnetic spectrum wavelength
Figure 4-3. A key to the scientific potential of the CDRL is the unprecedented integration of several technologies over a wide spectral range. Tunability, synchronization capabilities, and time resolution on the order of picoseconds are among the other important features of the proposed facilities. The lower-energy region of the infrared (different portions of which are of interest for different kinds of research) could be covered with chemical lasers; the proposed IRFEL would offer broad tunability over a wide range, in addition to high power.
Research in such areas as gas-phase reaction dynamics, combustion, isotope separation, and industrial processing is generally dependent upon advanced technologies and techniques. The CDRL will bring the key technologies together for the first time. At its heart could be an IRFEL, which has been the subject of a great deal of work by our group. The IRFEL, together with two ALS beamlines, optical lasers, and molecular-beam machines, will enable research that has a great impact on our understanding of pure and applied chemical dynamics.

The CDRL will occupy a new building adjacent to the ALS so that photon beams from both the ALS and the proposed IRFEL (which would go in a shielding vault in the basement of the new building) can be delivered to the experimental stations. The ALS beamlines have been another area of study and development by our group, in collaboration with the ALS staff and the Center for X-Ray Optics in LBL's Materials Sciences Division. Advanced optical lasers are being designed by colleagues from both the University of California at Berkeley Chemistry Department and Sandia National Laboratories. These cuibuborators have been deeply involved in the design of experimental facilities and in the development of the research program.

Since the original conceptual design was published in February 1990, we have revised it to incorporate an accelerator with $500-\mathrm{MHz}$ superconducting rf cavities (Figure 4-2). The primary reason for the move from the lessexpensive room-temperature technology was the user requirement for photon-beam wavelength stability. Their requirement for stability to within 1 part in $10^{4}$ translates into an electron-beam energy fluctuation of less than 5 parts in $10^{5}$. By reducing cavity losses due to wall resistance to nearly zero, the superconducting accelerator technology also allows continuous-wave (cw) operation. Pulses are desired for the CDRL application, so cw' operation is best interpreted as a means of increasing the average power, permitting more data to be obtained in a given time.
CDRL: A Unique Combination of User Facilities

IRFEL Design Progress 
Figure 4-2. Recent thinking about the CDRL IRFEL points toward a new design based on 500-MHz niobiumtitanium superconducting linac structures. The superconducting technology allow's continuous operation (with various pulsed modes and the ability to serve multiple experiments) and lends itself to a recirculation loop. Downstream of the accelerator apparatus shown here, coherent infrared radiation is produced with a variable-gap undulator and an optical cavity with a broadly tunable hole-coupled outcoupling scheme. Also shown here is an indication of how the new design might fit in the basement shielding vault of the CDRL building proposed earlier; the beam dumps are shown but the cryogenic systems, which would be outside the vault, are not.

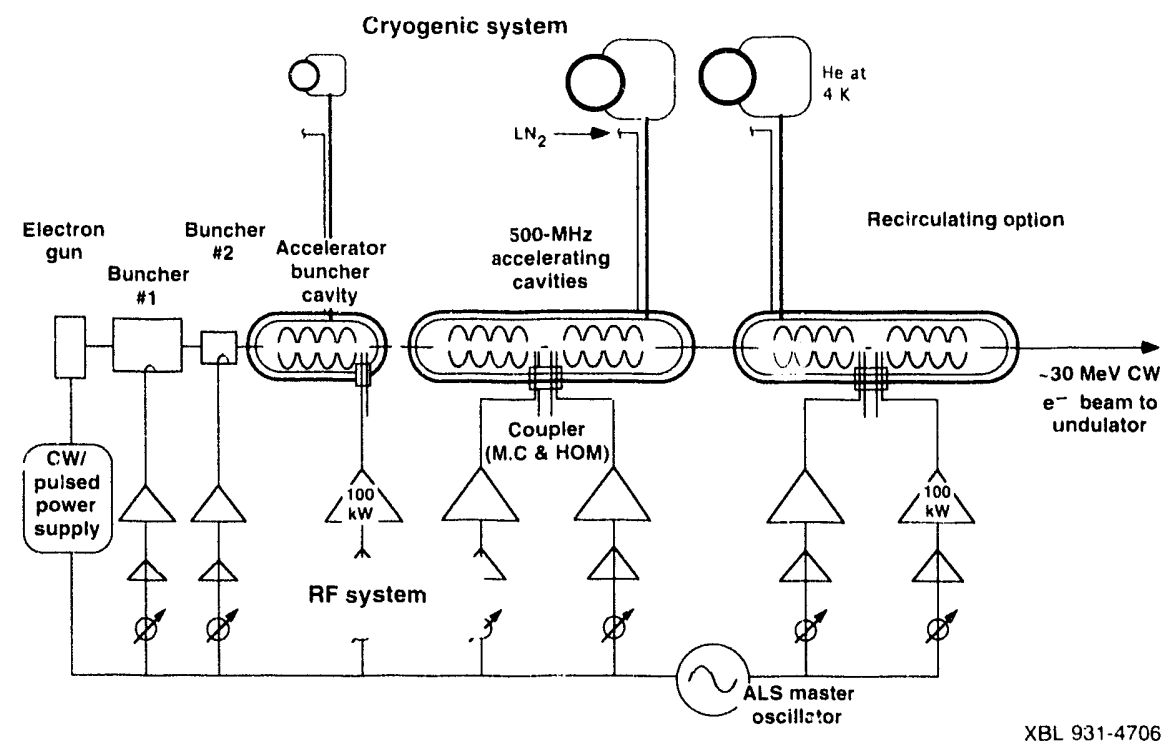

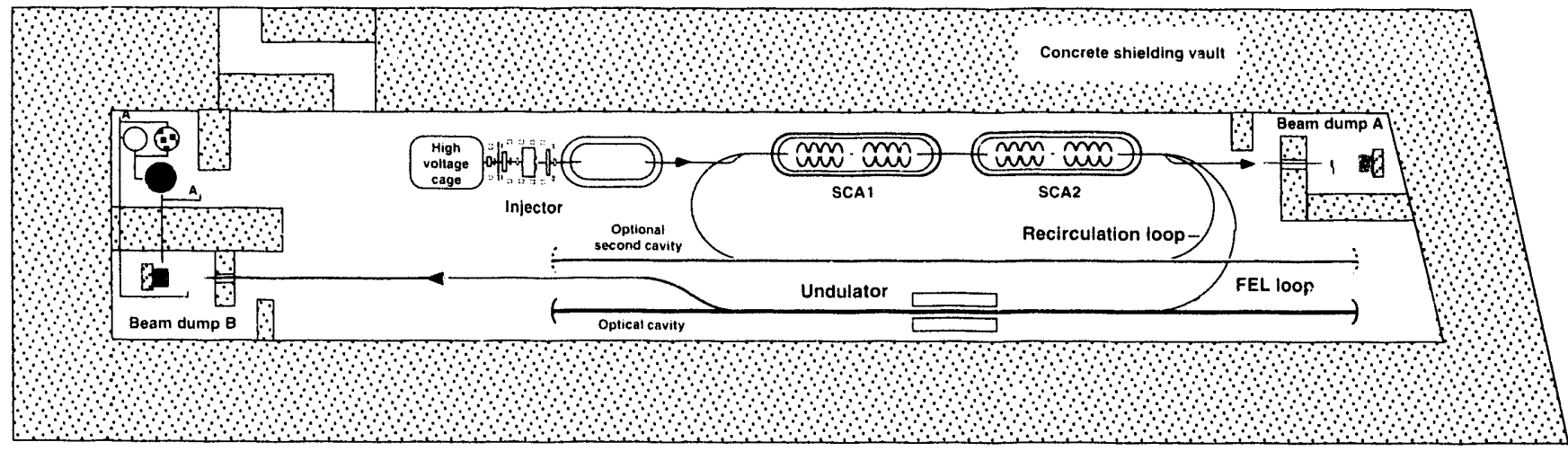

XBL 931.4707

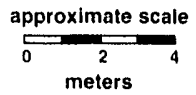

The pulse train can be tailored to meet the experimenter's needs in a variety of ways, including synchronization with the ALS pulses. This flexibility will allow simultaneous service to multiple users. The IRFEL's micropulse duration is $33 \mathrm{ps}$, with a repetition rate of $6.6 \mathrm{MHz}$. Infrared radiation is produced with a variable-gap undulator and a $24.6-\mathrm{m}-\mathrm{long}$ optical cavity with a broadly' tunable outcoupling scheme. Table 4-1 summarizes the parameters of the new IRFEL design.

Particular attention was paid to stability and tunability, which are crucial for the users' needs. A detailed analysis of the effect of the electron beam fluctuation upon the optical performance was carried out through analytic calculation and numerical simulation. Also studied were various sources of fluctuations in the gun, bunchers, and accelerating sections, as well as feedback and feedforward schemes to reduce these fluctuations. The superconducting design proved superior to the 1990 room-temperature design, which had an electron-beam energy stability of 1 part in $10^{-3}$. Thus the 500$\mathrm{MHz}$ superconducting design appears to be the best choice for our purposes. 
Table 4-1. Some Characteristics of the Proposed CDRL IRFEL

\begin{tabular}{lc}
\hline Accelerator & \\
RF frequency $(\mathrm{MHz})$ & 500 \\
Maximum energy $(\mathrm{MeV})$ & 55 \\
Micropulse & $0.35 \%$ at $55 \mathrm{MeV}$ \\
FWHM energy spread & 33 \\
FWHM duration $(\mathrm{ps})$ & 6.6 \\
Repetition rate $(\mathrm{MHz})$ & \\
Output & $3-50$ \\
Wavelength $\lambda(\mu \mathrm{m})$ & $10^{-4}$ \\
Line $:$ idth & $\leq 0.1$ \\
Bandwidth stability $\delta \lambda \lambda$ & 600 \\
Intensity stability $\delta \mathrm{I} / \mathrm{I}$ & transform-limited \\
Average power $(\mathrm{W})$ &
\end{tabular}

The FEL design must provide wide wavelength coverage while minimizing operational interruptions. At a fixed electron energy, the wavelength can be tuned by about a factor of two by varying the magnet gap of the undulator. For rapid fine tuning, we can change the electron beam energy by $\pm 1 \%$, varying the photon wavelength by $\pm 2 \%$. By operating the accelerator at any of four different energies $(55.3,39.1,27.7$, or $19.6 \mathrm{MeV})$ and using these tuning techniques $\mathrm{v}$ 're an cover a wavelength range of 3 to $50 \mu \mathrm{m}$.

The beam reacues about $30 \mathrm{MeV}$ in its first pass through the pair of superconducting rf cavities. With an extra, in-phase recirculation pass, it reaches about $55 \mathrm{MeV}$, greatly extending the short-wavelength capability of the IRFEL. In another operational mode, the recirculated bearn could instead be introduced into the cavities $180^{\circ}$ out of phase with the rf. This would decelerate the beam, putting its power back into the rf cavities in a sort of flywheel effect for use on the next pulse. The electron beam, and hence the optical beam, would become quite powerful.

Our work on the recirculation scheme is beginning to address such important issues as isochronous beam transport and safe dumping of energetic, intense beams. We and our potential users are also studying the scientific implications of operation at this high power. The design effort has spawned several experimental programs, including an LBL-Stanford collaboration on development of novel diagnostics for FEL optical pulses, a Stanford-LBL-TRW-BNL collaboration on optimization of superconducting cavities for FEL, an LBL experimental study of hole-coupling and resonator modes, and a joint LBL-CEBAF test bed for demonstrating superconducting IRFEL technologies.

The ALS injection complex includes a traveling-zuave linac that produces a $50-\mathrm{MeV}$ eleciron beam. After the storage ring has been filled, the injection complex will be idle for the useful lifetime of the stnied beam, which is expected to be several hours. A variety of interesting experiments could be conducted with that beam, including plasma focusing, tests of accelerator structures, and geveration of "chirped" photon pulses. Accordingly the Center is building the Test Beam Facility, a DOE-funded initiative using the ALS linac. Ti. facility will use the linac between injection cycles for a highly productive and cost-effective program in beam physics with minimal disruption to ALS operations.

\section{Trst Beam Facility}


Research Program
Many interesting experiments could be performed with this convenientiy available, short-pulsed, low-emittance electron beam. These two investigations are planned for the initial research program: a plasma focus and the generation of short $\mathrm{x}$-ray pulses through Compton scattering.

When a relativistic electron beam passes through a plasma, electromagnetic interactions focus the beam. To date, most work with the plasma-focus concept has involved thin "lenses." Continuous plasma focusing with thick lenses holds the promise of overcoming the so-called Oide limit-a fundamental limit of focusability arising from statistical emission of high-energy photons in a sharp focusing bend. Our plans include a proof-of-principle test and systematic exploration of plasma-focus ideas generated at our Center. One of the ideas is a long, continuous plasma focus in which diaphragms and differential pumping combine to taper the plasma density. The density will be tapered from about $1 \times 101^{0}$ to $5 \times 10^{12} \mathrm{~cm}^{-3}$ over a length of $0.5 \mathrm{~m}$. We hypothesize that, at $50 \mathrm{MeV}$, such a device could focus a beam with $13-\mathrm{mm}$ cross section into a $400-\mu \mathrm{m}$ spot. Our scaled proof-of-principle work will involve plasma lengths ranging from 10 to $50 \mathrm{~cm}$, with density tapering from about $1 \times 10^{11}$ to $5 \times 10^{13} \mathrm{~cm}^{-3}$ over that distance.

Two requirements must be satisfied for an effective plasma focus: the plasma response time must be short compared to the pulse length, and the plasma return currents within the beam must be small. We have calculated parameters for a number of experiments that can be performed using the 50$\mathrm{MeV}$ injector; they will allow careful study of these requirements in both underdense and overdense plasmas." Furthermore, a study of the effect of plasma return currents on the effectiveness of the focusing can provide insight into the usefulness of plasmas in reducing beam-beam interaction.

The design of the plasma source and diagnostics is currently under way. Two candidate sources are an rf discharge source and a photoionization source driven by an excimer laser. The plasma properties will be measured using Langmuir probes and a $65-\mathrm{GHz}$ Michelson interferometer.

Another experiment at the facility will use the ALS linac's beam for an ALS-like purpose: the generation of $x$-rays. Today, the shortness of photon pulses that are produced by either interaction with a magnetic field (synchrotron radiation) or interaction with visible photons (Thomson scattering) is limited by, and comparable with, the shortness of the electron beam. For the ALS linac beam, the shortest photon pulses from a direct collinear interaction would be a few tens of picoseconds long. We have recently hypothesized that a third approach could break through this limit, producing sub-picosecond $x$-ray pulses.

The new approach, being supported with Laboratory Directed Research and Development funds, is based upon $90^{\circ}$ Compton scattering with a visible laser (Figure 4-4). In this configuration, the shortness of the $x$-ray pulse is limited not by the length of the electron pulse, but rather by the length of the laser pulse or the transit time of the laser pulse across the waist of the focused electron pulse. Therefore it is crucial to focus the electron beam to a narrow waist matching the laser pulse length. A short-pulse solidstate laser $\left(\tau_{\mathrm{L}}=200 \mathrm{fs}, \mathrm{E}=100-200 \mathrm{~mJ}\right)$ is being designed by the femtosecond laser laboratory in LBL's Materials Sciences Division. Also being designed is an $\mathrm{x}$-ray detector that offers femtosecond time resolution. In cooperation

\footnotetext{
" $T$ l a terms "overdense" and "underdense" indicate whether the plasma is denser than the particle beam or vice versa.
} 


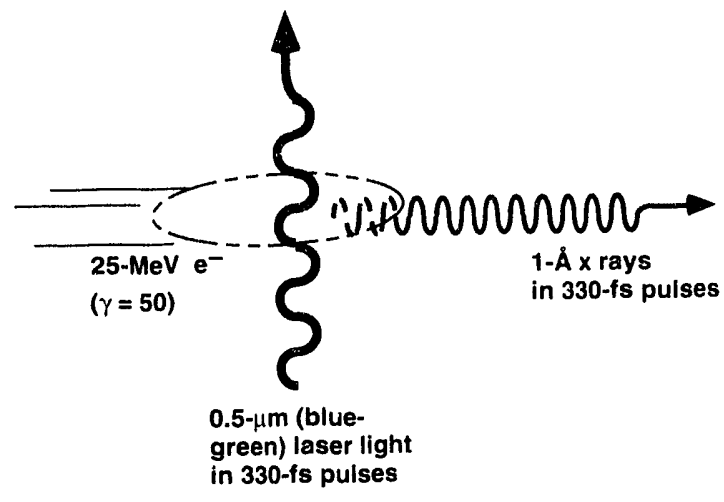

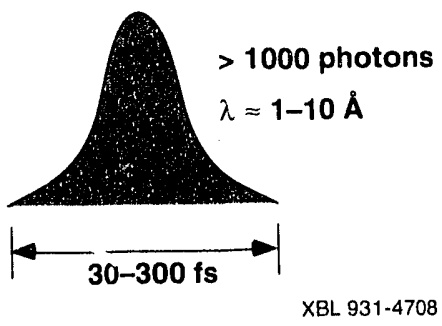

Figure 4-4. One of the most intriguing experiments for the Advanced Test Beam Facility is production of sub-picosecond $x$ ray pulses through $90^{\circ}$ Compton scattering of a visible laser beam against the $50-\mathrm{MeV}$ electron beam. with LBL's Center for X-ray Optics, we are examining ways to direct the beam onto detectors and experimental apparatus. We are also designing the beamline components required to focus the electron beam to a 70-100 $\mu \mathrm{m}$ spot and then separate it from the $x$-rays after the interaction point. With the current design parameters, we should be able to produce a 100-300 fs $x$-ray pulse, containing about $10^{5}-10^{6}$ photons, with a wavelength that can be varied in the range of 1-10 $\AA$ by changing the electron-beam energy.

A variety of other experiments will also be made possible by the facility, including beam-structure interaction studies, investigations of beam-conditioning cavities for FELs, and the "chirping" of conveniently long (10-ps) electron-beam bunches to produce photon pulses much shorter than thiat.

Figure 4-5 shows the overall layout of the Test Beam Facility. We have been designing the beamline components while studying the proposed experiments and deciding how best to reconcile their somewhat different implications for the magnetic lattice of the transfer line. For example, the plasmafocus experiment requires several transverse measurements of the electron beam (which therefore should be perturbed as little as possible downstream of the focus), whereas the Compton-scattering experiment requires separation of the electron and $x$-ray beams downstream of the interaction point.

We anticipate that most experiments will be entirely transparent to ALS operations, involving no changes in the electron-gun and linac settings. Some special experiments might call for temporarily changing the relative amplitude and phase settings of the two linac tanks; others might require the gun pulser and the grid voltage to be turned up to their maximum capacity in terms of charge extraction and pulse-train length. The linac will remain under the overall control of the ALS throughout.
"A term for a small, rapid change in energy during a pulse, historically based in radio transmission of Morse code.
Facility and Operations 
Figure 4-5. Because the ALS is based on a storage ring, the injector linac will be idle much of the time. This affords a highly cost-effective opportunity to develop a facility for beamphysics research. This diagram shows the probable layout of the Advanced Test Beain Facility, which is now moving from design into construction. The facility will support a variety of experiments, such as plasma focusing of an electron beam and the production of ultrashort $x$-ray pulses using $90^{\circ}$ Compton scattering of a visible laser beam off an electron beam.

\section{Optics Experiments}

\author{
Bench Testing of Holl,
}
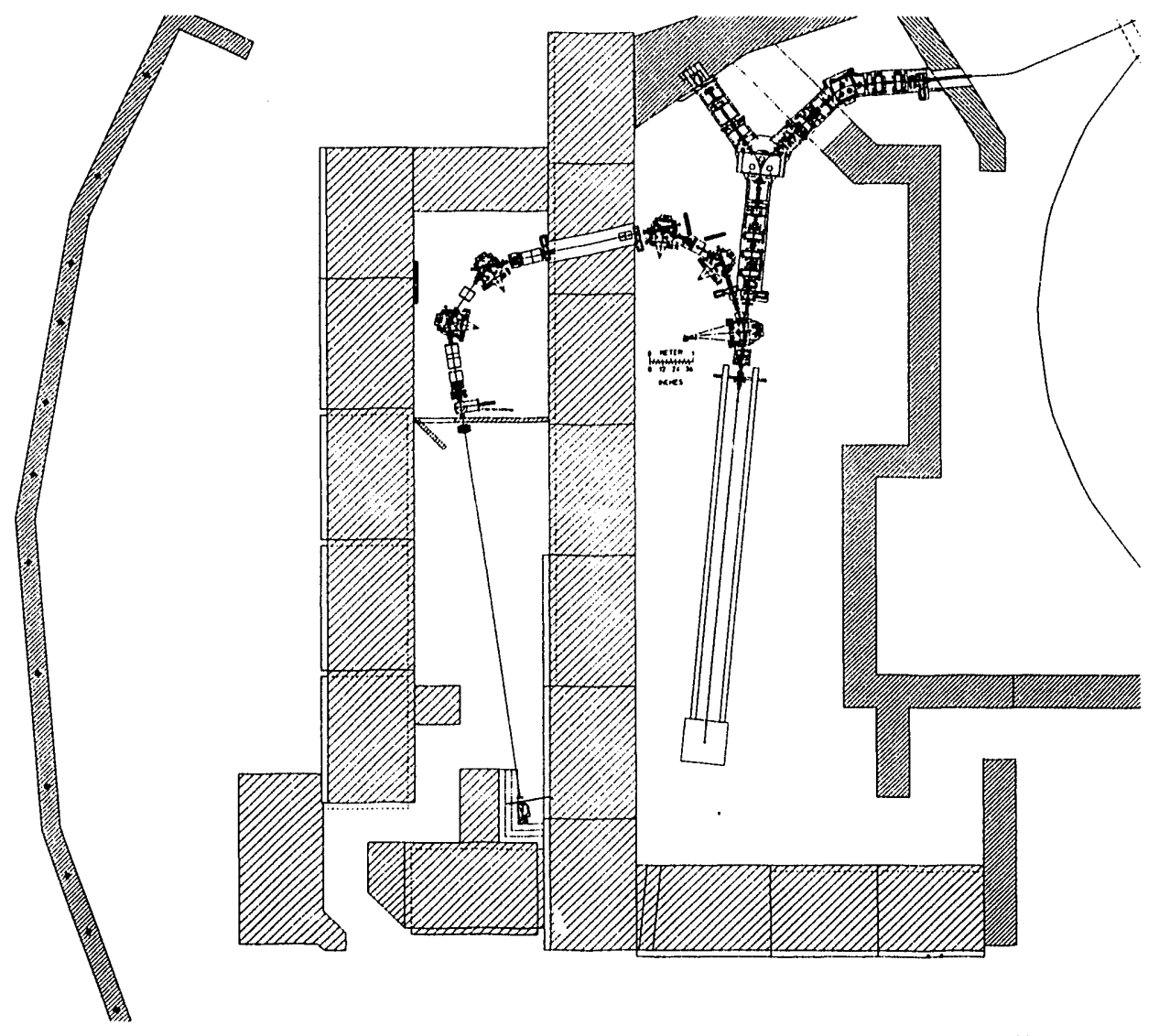

XBL. 9111-6852

The Center for Beam Physics has greatly expanded its experimental capabilities with a new optics laboratory that will serve many efforts, including the design of the proposed CDRL IRFEL and the development of optical components for the Test Beam Facility described in the previous section. Two projects have been carried out thus far: bench testing of the Fox-Li code Hol.D and development, together with Stanford University, of advanced optical diagnostics for FELs.

Hole coupling was selected for the CDRL IRFEL's optical resonator after extensive computer simulation, primarily using the code HoLD. To validate these simulations, we performed scaled experiments by injecting a visible HeNe laser beam into a stable cavity, as shown in Figure 4-6. We studied two cases: a Gaussian near-concentric symmetric resonator and a holecoupled resonator with degenerate higher-order modes. The first case, with its simple geometry, allowed direct comparison with analytical results and HoLD output and was also useful for benchmarking the diagnostic equipment. The second case provided an effective means of exercising the code and also yielded intrinsically useful results, since mode degeneracy should be avoided for good FEL operation. 


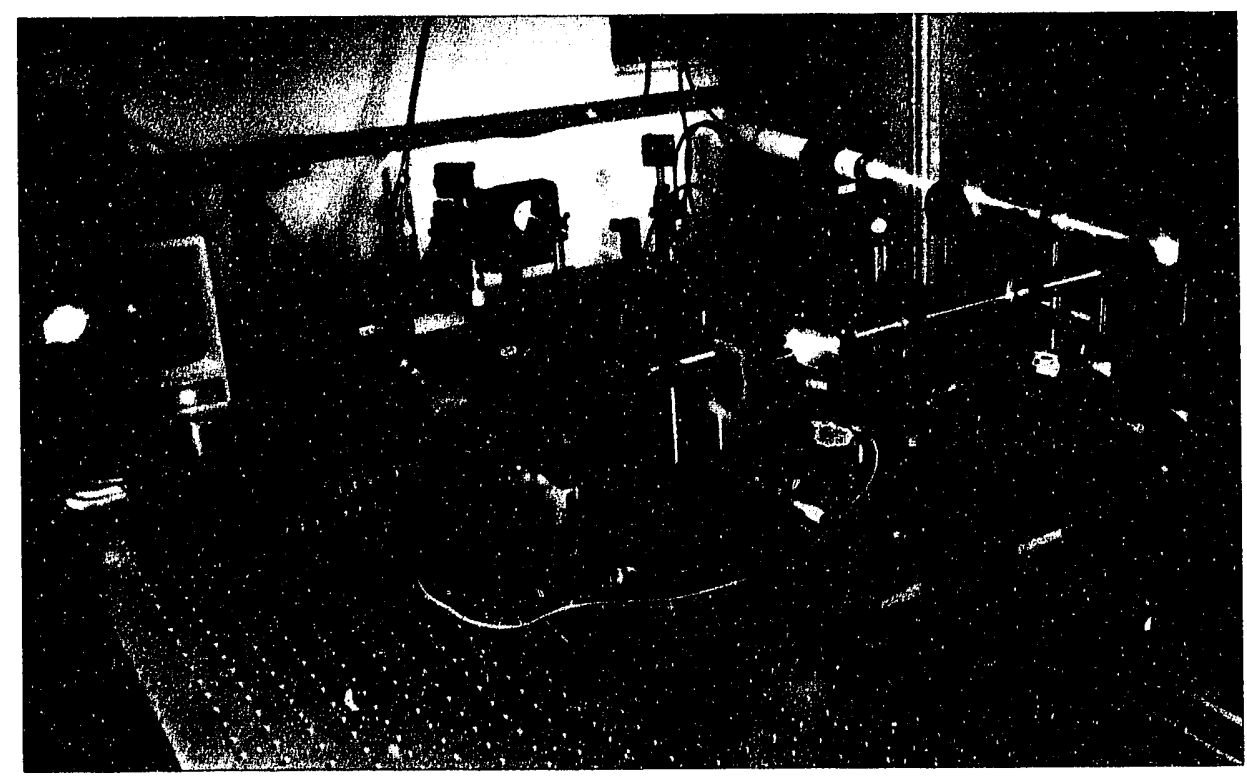

CBB 928.6565
Figure 4-6. To validate analytical solutions and code simulations, we performed scaled experiments by injecting a visible HeNe laser beam into a stable cavity. The experiments have enabled us not only to verify the Fox-Li code known as HoLD, but also to gain some insights into avoidance of degenerate higher-order modes in FELs.
The measurements agreed reasonably well with HOI.D calculations of intracavity and outcoupled mode profiles and other parameters. We observed mode degeneracy, as evidenced by an intracavity mode profile that alternated between two higher-order modes, and related it to our deliberate choice of stability parameter, outcoupling hole size, and intracavity aperture size. Future experiments will concentrate on improving the laser stability, suppressing cavity-mirror vibrations, and achieving better pointing accuracy so that we can accurately study the actual design parameters of the CDRL IRFEL.

Working with colleagues from Stanford University, we designed a novel diagnostic system to measure the spectrum and pulse width of an IRFEL's output. By using an image dissector and a high-speed single-element detector with integraing sphere, the system provides spectral and temporal information for each micropulse within the pulse train. The system is shown schematically in Figure 4-7.

Key elements for the spectral diagnostics are the mode-matching telescope, high-resolution spectrometer, imaging telescope, and image dissector. The imaging telescope, located between the spectrograph and the image dissector, varies the magnification of the image that arrives at the dissector, thus allowing the desired spectral resolution to be selected. For pulse-width measurement, the system uses single-pulse autocorrelation through non-collinear optical mixing in a frequency-doubling crystal, along with the imaging telescope and the image dissector. Here the image is that of the region of the nonlinear crystal in which second-harmonic light was generated.

After the spectrometer system was developed in our laboratory, we tested it using an FEL beam at Stanford in September and October. Preliminary results indicate that the single-element detector with integrating sphere

\section{Advanced Optical Diagnostics for FELs}




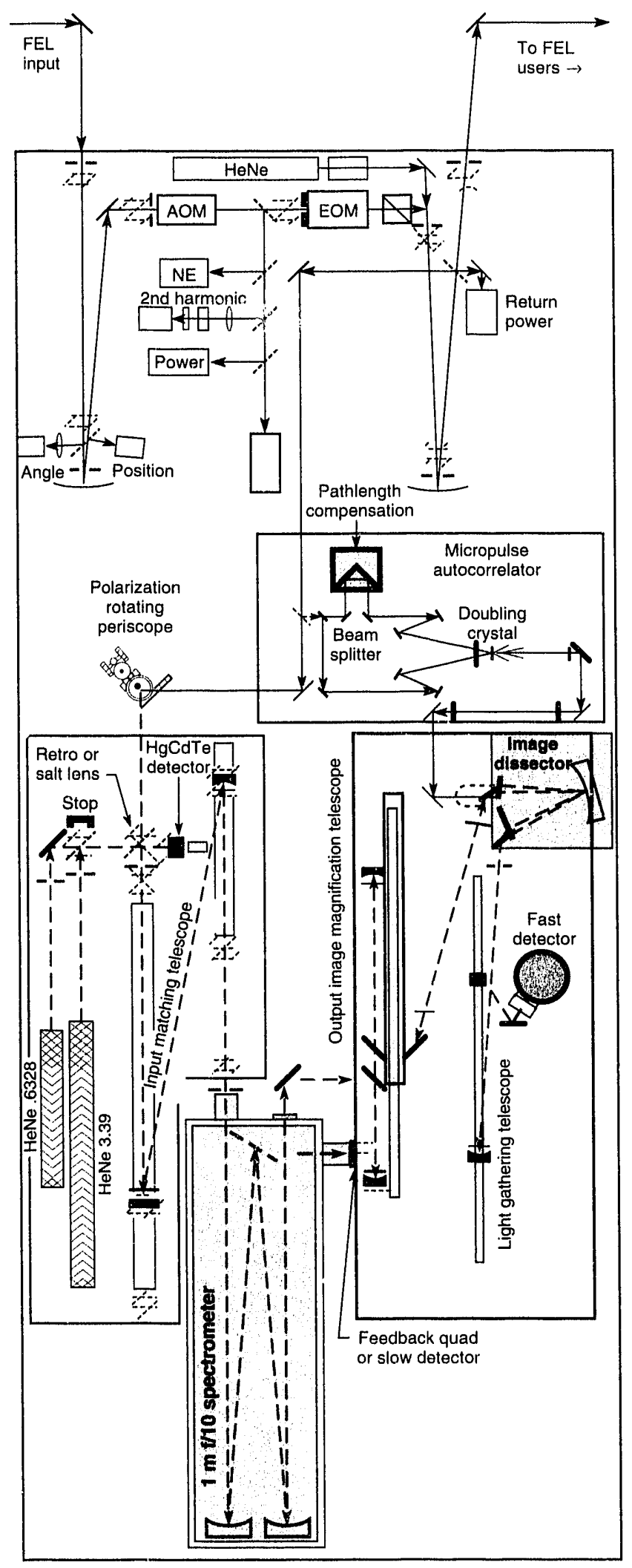

diagnostics has resulted in a system that can perform both spectral analysis and pulse-width measurement of the optical output. Preliminary results from testing with an XBL 9212.5858 FEL beam at Stanford have been encouraging. 
has the requisite sensitivity and rise time. A micropulse spectrum with 5 spectral "bins," or sets of data in different parts of the spectrum, was obtained. Further improvements to the spectrometer and its user interface will be tested in another FEL run at Stanford early in 1993, and we are also about to begin building the single-pulse autocorrelator system for pulse-width measurements.

Members of the Center for Beam Physics have been involved in the Advanced Light Source from the outset, focusing primarily on the immediate needs of the project but also investigating many basic physics issues involving high-brightness electron storage rings that have numerous insertion devices. Much of this research is highly generic and is relevant, for the most part, to any third-generation source, as well as to storage-ring-based free-electron lasers and to compact damping rings envisioned for high-energy linear colliders.

Because of the high beam current and short bunch length in the ALS, it is important to minimize the beam coupling impedance of the vacuum-vessel components. If this impedance were high, it might excite coupled-bunch and single-bunch instabilities, and the electromagnetic energy deposited in the vacuum vessel by the beam (hundreds of kilowatts are potentially available) might cause excessive heating. Our Beam Electrodynamics group has studied many ALS structures, beginning at the design stage, a process that includes computer modeling and measurements of some actual components by launching waves down a coaxial wire. Our most recent achievement was measuring the higher-order modes of a spare ALS rf cavity. With these measurements we determined the effectiveness of damping (which is accomplished with high-pass filters connected to the input power waveguide) and obtained the data necessary for accurate simulation of coupled-bunch motion.

The information is being put to use in the design of damping systems. In the ALS, bunch-by-bunch damping schemes will be implemented for all three axes so that errors in the position and phase of each bunch can be measured and corrected. Computer simulations and calculations of the beam behavior, which used the data on the higher-order modes of the rf cavity, suggest that the beam instabilities can be safely cortained with the proposed feedback systems. Tests of the longitudinal feedback system are planned on the ALS in the near future. This system was developed in cooperation with SLAC, and a similar scheme will be used for the PEP-II collider described earlier in this chapter.

The device that will apply the feedback to the beam is a "kicker," which consists of a pair of coaxial electrodes approximately a quarter-wavelength long connected by half-wavelength delay lines. This structure uses only onefourth the power of a single-electrode design for a kick of the same amplitude. Low-power measurements of a prototype confirm the predicted performance, and a production unit is being made. Transverse kickers are also being designed.

\section{Accelerator Physics for the ALS}

RF Measurements and Feedback Systems 


\section{Injector \\ Commissioning \\ Experience}

\section{Beam \\ Electrodynamics}

\author{
B-Factory \\ Contributions
}

Members of our Center working on the ALS project have been closely involved in the commissioning of the $50-\mathrm{MeV}$ injection linac and the $1.5-\mathrm{GeV}$ booster synchrotron. This process (see Chapter 3 of the AFRD Summary of Activities) is essentially complete; we are now studying the beam dynamics of the injection system and taking as many opportunities as possible to gain operational experience with it. By building our understanding of this complicated system, we will be able to commission the storage ring efficiently in early 1993 and then provide smooth, reliable operations after the facility opens to users.

As greater demands are made on the performance of accelerators-such as increased luminosity, as in PEP-II, or lower emittance, as in the ALS-it becomes ever more important to understand potentially disruptive of phenomena within the beam chamber and to perform various of "gymnastics" to monitor and control the beam. An area of special interest is the understanding and control of the potentially disruptive electromagnetic interaction between the beam and the conductive zoalls of the vacuum chamber and various devices. The Beam Electrodynamics group within the Center approaches these problems through analysis, simulation, and experimentation. In 1992 they contributed to PEP-II by leading the design of rf and feedback systems, analyzed beam impedance and feedback issues at the ALS, and continued their history of contribution to the Tevatron by studying a stochastic beam-cooling upgrade.

The major rf-design challenge posed by PEP-II is control of coupled-bunch motions. In each of the three directions (horizontal, vertical, and longitudinal), these motions have 1658 modes that may be driven strongly by the higher-order resonances of the rf cavities. Each higher-order cavity mode can drive a hundred or so of these motions at a growth rate thousands of times faster than the damping that naturally occurs in the accelerator. The first step toward stabilization is to reduce the shunt impedances of the higher-order modes by several orders of magnitude without corresponding degradation of the desired fundamental mode. Removal of the remaining instabilities will then be within the reach of a practical feedback system. To reduce the shunt impedance of the higher-order modes, we attach waveguides to the cavity to couple these modes to an external resistor.

Figure 4-8 shows a design for such a cavity and a low-power prototype, designed and analyzed with the aid of the MAFIA code and Kroll-Yu processing of the output. Extensive measurements designed to examine which modes are damped and whether there is interference with the fundamental mode have shown good agreement with expectations; for example, the strongest longitudinal mode, $\mathrm{TM}_{011}$, was predicted to have a loaded $\mathrm{Q}$ of less than 30 and was measured to have a loaded $Q$ of approximately 28 (suppressed by more than three orders of magnitude from the unloaded case). We developed a bead-pull perturb-tion apparatus to measure the impedances of the cavity modes and to map field profiles for mode identification. We are now designing a nigh-power test model to verify fabrication and 


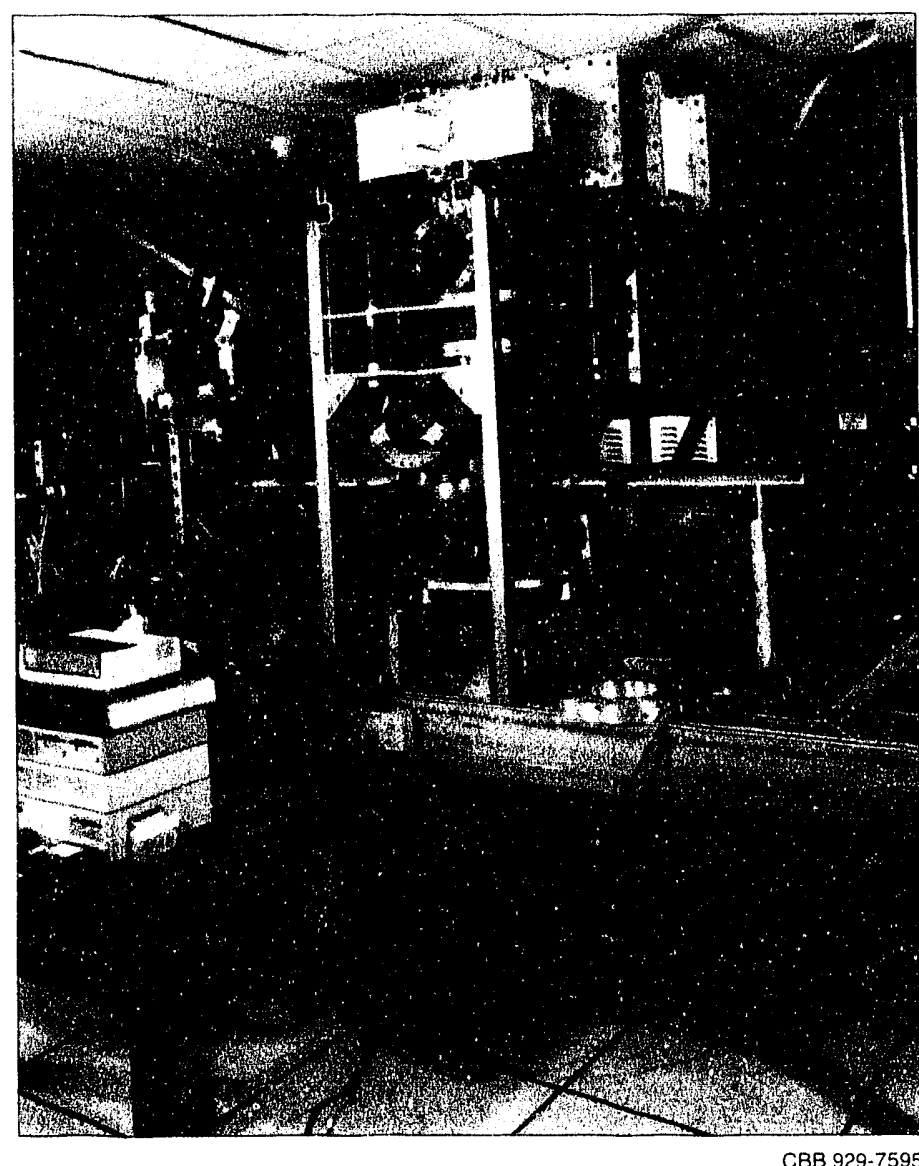

CBB 929-7595

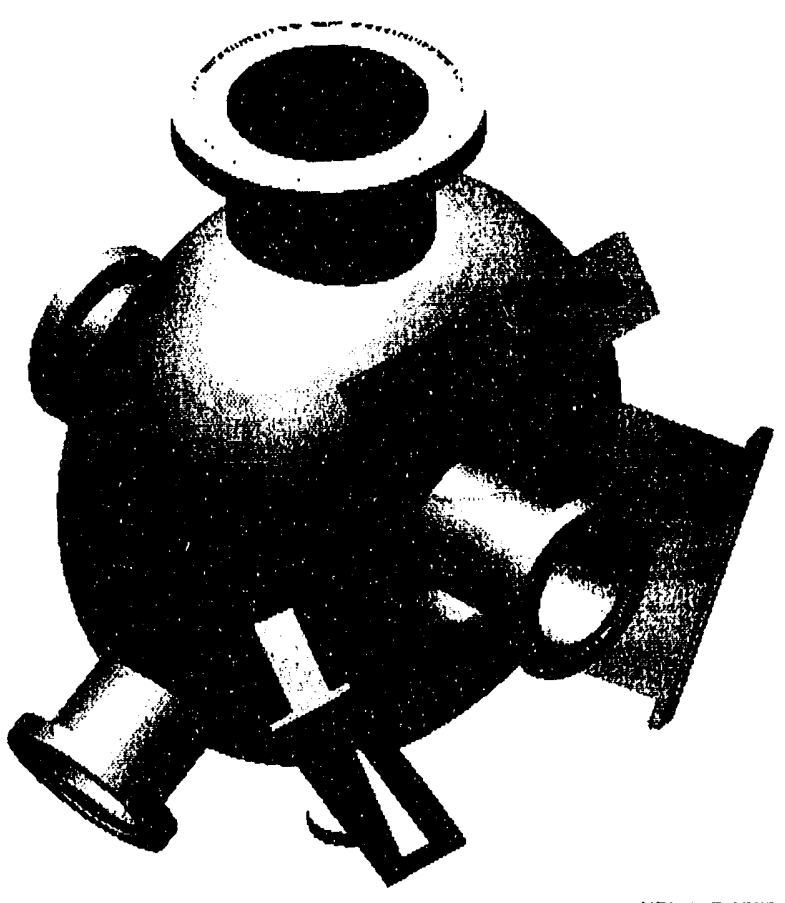

Figure 4-8. This design for the PEP-II rf cavities was studied extensively in computer simulation. Tests on the low-power prototype shown here have led us to begin the design of a high-power prototype. The design is intended to accelerate the beam with the fundamental mode while diverting the disruptive higher-order modes into external resistors.

conditioning techniques and to study operation at a continuous power level of $150 \mathrm{~kW}$. It will also be used for further studies of the external loads that dissipate the higher-order-mode power.

Coupled-bunch modes that fall within the width of the fundamental resonance cause an additional driving impedance. This problem, endemic to large-diameter rings, must be addressed with active rf feedback around the cavity and its driver, a problem that we are now studying in collaboration with SLAC and LLNL. The design of the longitudinal feedback system is proceeding in collaboration with SLAC, and work on the transverse feedback system is about to begin. Computer simulations and calculations of the longitudinal beam motion, using the measured higher-order-mode data, have shown that the specified feedback system can safely contain the instabilities excited under the expected worst-case conditions, which occur at injection. The effects of noise on the system are being investigated. It appears that the problem of suppressing coupled-bunch modes, although difficult, can indeed be solved. 


\section{Fermilab Antiproton Cooling System}

\section{FEL Accelerating Cavities}

The latest achievement in our ongoing collaboration with Fermilab is the design of a biplanar electrode system for more rapid cooling of the beam for the antiproton source.* (LBL was involved in the initial design of pickup and kicker electrodes for this cooling system and has been continually engaged in analyzing the system's performance and seeking ways of improving it.)

In earlier years we had demonstrated that, for power-limited cooling systems, it is more efficient and cost-effective to double the number of cooling electrodes than to double the operating frequency, whereupon we developed biplanar electrodes that could effectively double the number of electrodes without using any more space. This scheme, with the existing 2-4 $\mathrm{GHz}$ electronics, appeared to yield better results than would a system with uniplanar electrodes and completely reworked $4-8 \mathrm{GHz}$ electronics. Calculations indicate that the resulting performance would exceed the needs of any anticipated upgrade to the Tevatron complex, including the proposed new main injector. The validity of our beam-cooling calculations was affirmed by comparing the results with cooling data from Fernilab. In 1992, we completed a detailed design and cost estimates for the electrode system and began studying the performance of a prototype module. Full production awaits the results of the study and a go-ahead decision by Fermilab.

As mentioned in the earlier section on the proposed CDRL IRFEL, minimization of fluctuations in the energy and intensity of an FEL requires stringent maintenance of electron-beam stability. This in turn has implications for the rf cavities in the accelerator and other aspects of the accelerating system. For instance, coupled-bunch motions driven by the higher-order modes of the rf cavity may be excited in a recirculating beam, causing $\mathrm{rf}$ voltage fluctuations that make the energy of the beam fluctuate. As part of our collaboration with Stanford, Brookhaven, and TRW, we have performed various experiments in FEL technology.

A two-cell superconducting rf cavity, similar to a cavity of the IRFEL, has been studied extensively with our bead-pull perturbation apparatus in search of higher-order modes. These modes can be damped by putting probes at appropriate locations in the beampipes at the ends of the cavity and connecting them to external resistive loads. A similar copper cavity has been used to study a network for input impedance matching and phase adjustment. In 1993, together with Stanford, we will measure the quality factors of the hi rher-order modes of this niobium cavity at superconducting temperatures.

Of the many ideas that have been proposed for the electron-positron colliders of the next century, the two-beam accelerator, or TBA, appears to be one of the more promising. Figure 4-9 illustrates the concept. The first of the two beams is a "drive" beam, generated by an induction linac, that has high current but relatively low energy (perhaps $3 \mathrm{kA}$ at $10 \mathrm{MeV}$ in a full-scale TBA). This beam is passed through either an 'ndulator-based $F E L$ or a relativistic klystron (RK), generating microwuve power on the order of $1 \mathrm{GW}$ per meter of length. The powver is applied to an adjacent high-gradient acceleration structure, which accelerates a second electron beam to

\footnotetext{
"Beam cooling means measuring the "temperature" or internal motion of the particles in the beam and applying feedback to reduce this motion, thereby increasing intensity.
} 


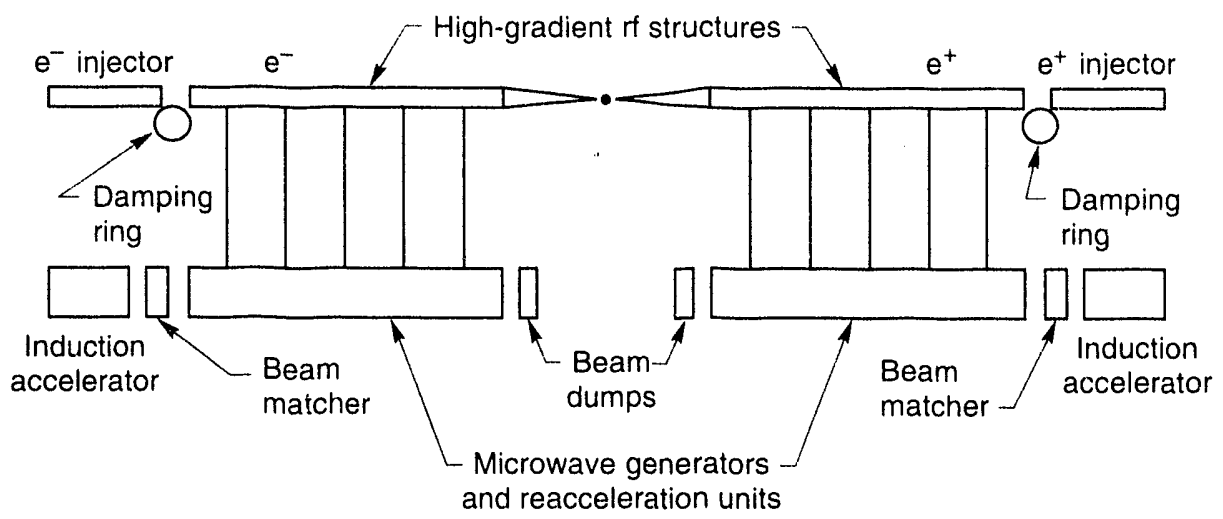

XBL $901-5730$

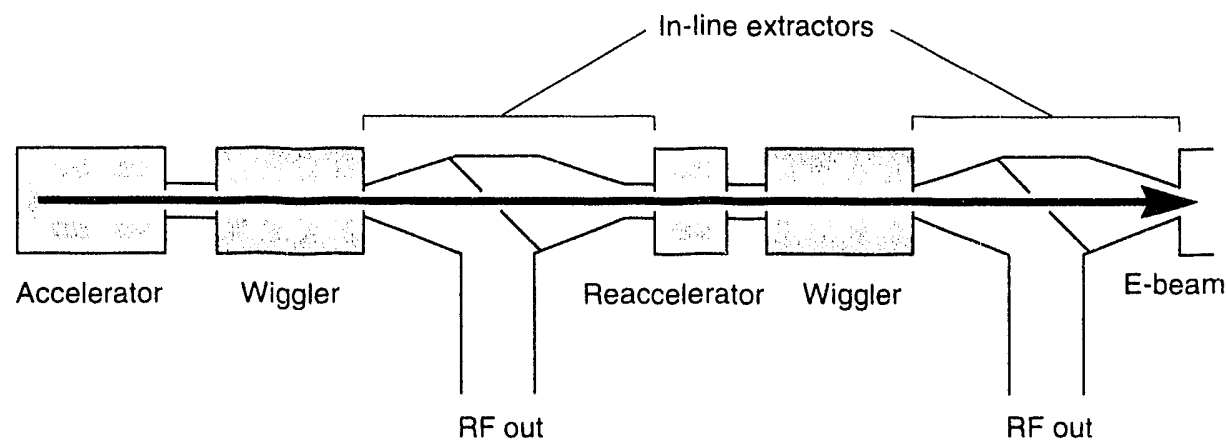

XBL $901-5729$

high energy. Today, the TBA technology is in the early stages of development; designs are being developed and evaluated by researchers in the Center's HighEnergy Collider Physics Group, in collaboration with colleagues from LLNL and from KEK, the high-energy physics laboratory in Japan.

Initially the TBA/RK work involved longitudinal bunching of the drive beam. This is adequate for low energies, but at moderate energies (greater than $3 \mathrm{MeV}$ or so) it becomes less effective. To extend our work to higher energies, we have been experimenting with a transverse chopper cavity or "choppertron," built according to our designs by Haimson Research. In 1991 trials, the choppertron produced impressive peak power-some 400 MW-but the pulses at such power levels were less than $10 \mathrm{~ns}$ in length. We determined that the problem was beam breakup caused by a spurious higher-order mode generated in the output structure at $13.6 \mathrm{GHz}$. We have since added a damping structure (Figure 4-10) that removes this higher-order mode; in 3-MeV experiments on the Advanced Technoiogy Accelerator at LLNL, we obtained 30 -ns pulses at $120 \mathrm{MW}$. These pulses had a phase jitter of about $2^{\circ}$, which implies good spectral purity. This satisfying demonstration of high power output from an RK provides a good basis for our continuing $R \& D$ program. We are investigating damping structures that could remove the higher-order mode without damping the desired mode, perhaps enabling us to simultaneously achieve the hundreds-of-megawatts power and the tens-of-nanoseconds pulse lengths.
Figure 4-9. As shown in the TBA sketch abov', a high-current, lowenergy drive beam is used for generating $\mathrm{rf}$ power that is applied to a high-gradient acceleration structure, where a low-current load beam is accelerated to high energy. The diagram below shows the progress of the drive beam through the rfgenerating devices (FEL wigglers in this example) and the reacceleration units that replenish the drive beam in between.

\section{Transversely Modulated RK}




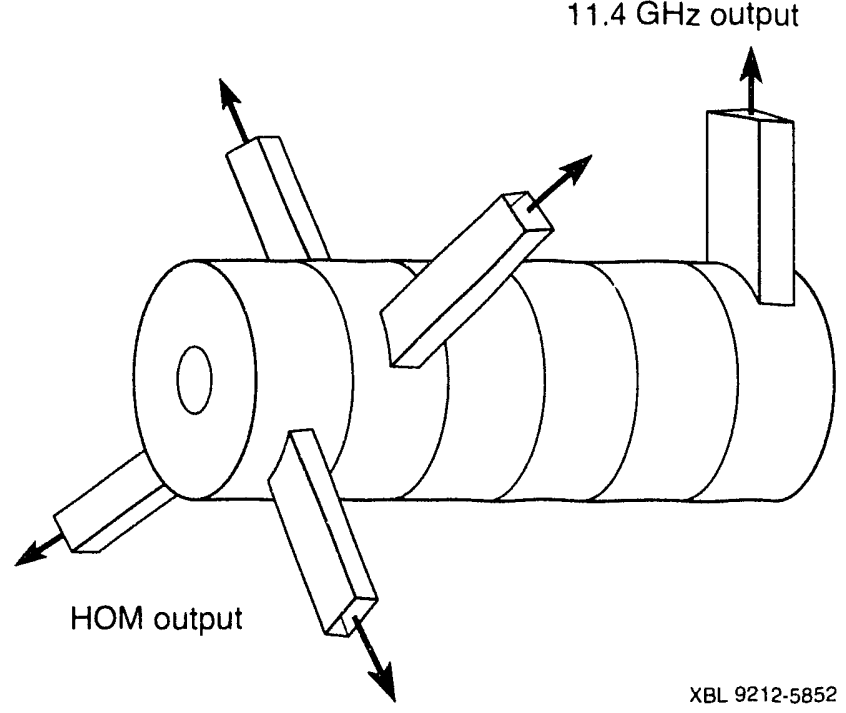

Figure 4-10. When the choppertron was equipped with this structure, a highly disruptive higher-order mode was effectively damped, enabling a 30-ns pulse of $11.4 \mathrm{GHz}$ microwaves at 120 MW. The low phase jitter (about $\pm 2^{\circ}$ ) indicates good frequency stability. The next step is to improve the efficiency of the damper so that the power level in the fundamental mode returns to the hundreds-of-megawatts level achieved (in much briefer pulses) without the damper. (After LLNL illustrations)
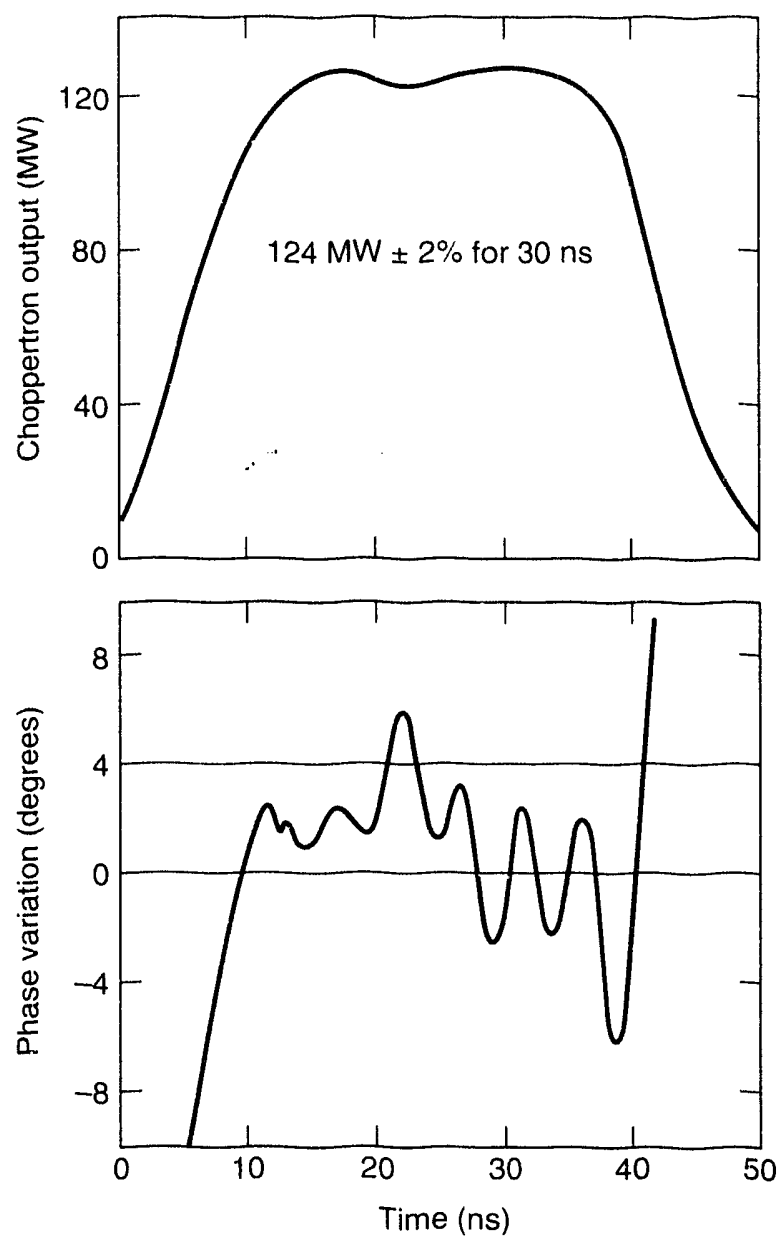

XBL 9212-5852
Standing-Wave FEL

Horizons for the TBA
The FEL, explored in our original TBA research, remains a proven candidate with great potential. We are developing an idea, called the "standing-wave FEL," in which the radiation is trapped in a standing-wave rf cavity and beat-coupled to a nearby high-gradient acceleration structure. This concept is currently undergoing intense analysis and theoretical study, with no experimental program anticipated in the near term.

The work done on the TBA since we conceived of it 10 years ago has validated the basic concept and the use of either an RK or an FEL as the source of rf power. However, there remains a vast amount of research and development before the TBA can be used in high-energy physics. Here are some of the planned near-term investigations.

- Re-acceleration of the "spent" drive beam (useful for economic reasons) will be examined in a planned 1993 experiment at LLNL.

- There is much theoretical and experimental work to be done in extraction of microwaves from the power source. A demonstration of repeated extraction is being studied at LLNL. 
- Sensitivity studies to determine the importance of various parameters will be important. A great deal of theoretical work has been done; coming years will see more studies on real apparatus.

- Economic matters will be significant in the eventual decision on whether to build a full-scale TBA and in the technological choices within such a project. We are working with LLNL on these issues.

A collaboration with KEK is under way, using an FEL from which up to 30 MW can be extracted at $8.6 \mathrm{GHz}$.

The gain of a free-electron laser or other resonant electron-beam device is limited by the spread in longitudinal velocity and, hence, the energy spread and emittance of a three-dimensional beam. The electron-beam emittance must be less than the wavelength of the radiation from the device divided by 4. In practice, the energy spread of the beam is often small, so the beam could be "conditioned" with special rf cavities. These cavities would impart more acceleration to the particles traveling longer paths, reducing the spread in longitudinal velocity. We have analyzed this idea with a simple numerical model for beam transport, assuming ideal rf cavities. We have also analyzed an FEL to evaluate its performance with reduced axial-velocity spread; these studies lead us to expect distinct improvements from beam conditioning. During 1992 we computationally analyzed a cavity geometry that promises to greatly increase the beam-cavity coupling. Experiments to test the feasibility of a beam-conditioning cavity are being planned for the Accelerator Test Facility at Brookhaven National Laboratory.

W. Barletta (LLNL), A. Sessler, and L. Yu, "Physically transparent formulation of a freeelectron laser in the linear gain regime," 14th International Free Electron Laser Conference (Kobe, Japan, 1992); LBL-32221 (1992).

W. Barry, J. Edighoffer, and S. Chattopadhyay (LBL) and S. Fornaca (TRW), "Field profile and loading measurements on higher order modes in a two cell $500 \mathrm{MHz}$ superconducting structure," 16 th International Linac Conference (Ottawa, Ontario, Canada, 1992); LBL_-32178a (1992).

V.G. Baryshevsky and 1. Dubovskaya, "Diffraction phenomena in spontaneous and stimulated radiation by relativistic particles in crystals (review)," LBL-31695 (1991).

I. Ben-Zvi, L.-H. Yu, R. Govil, and A.M. Sessler, "A proposed experiment for beam conditioning," 14th International Free Electron Laser Conference (Kobe, Japan, 1992); Brookhaven National Laboratory report BNL-47857 (1992).
S. Chattopadhyay and K.-J. Kim, "Generation of sub-picosecond x-rays," Workshop on the 4th Generation Light Sources (Stanford, CA, 1992); LBL-31968 (1992).

Y.-H. Chin, "Beam-beam dynamics during the injection process at an asymmetic ring collider," abstract for the Spring Mecting of the American Physical Society (Washington, D.C., 1992); Lawrence Berkeley Laboratory report LBL-31754a (1992).

Y.H. Chin, "Effects of parasitic beam-beam interaction during the injection process at the PEP-II B factory," in Procerdings of the International Conference on B Factories: The Siriti of the Ait in: Arcelerators, Detectors, and Physics (Stanford, CA, 1992); LBL-32468 (1992).

S. Chattopadhyay, K.-J. Kim, R. Byors, R. Donahue, J. Edighoffer, R. Gough, E. Hoyer, W. Leemans, J. Staples, B. Taylor, and $M$. Xie, "Design of a superconducting linearar accelerator for an infrared free electron
Beam Conditioning Publications and
Presentations 
laser of the proposed chemical dynamics research laboratory at LBL," 16th International Linac Conference (Ottawa, Ontario, Canada, 1992); LBL-32182a (1992),

Y-H. Chin, "Simple formulae for the optimization of the FEL gain length as a function of emittance, betatron wavelength and energy spread," 14th International Free Electron Laser Conference, (Kobe, Japan, 1992); LBL-32249 (1992).

Y.H. Chin, K.-J. Kim, and M. Xie, "Calculation of 3-D free electron laser gain: comparison with simulation and generalization to elliptical cross section," 14th Free Electron Laser Conference (Kobe, Japan, 1992); LBL-32286 (1992).

Y.H. Chin, K.-J. Kim, and M. Xie, "Threedimensional free electron laser theory including betatron oscillations," submitted to Phys. Rev. A; LBL-32329 (1992).

J.N. Corlett and J.A. Clarke, "New injection kicker magnets for the Daresbury SRS," in Procedings of the 3rd European Particle Accelerator Conference (Berlin, Germany, 1992), pp. 1469-1471.

J. Dorfan and A. Hutton (SLAC), M. Zisman (LBL), and W. Barletta (LLNL), "PEP II asymmetric B-factory: $R \& D$ results," 3rd European Particle Accelerator Conference (Berlin, Germany, 1992); LBL-32098a (1992).

I. Dubovskaya, "Coherent generation of visible radiation by relativistic electron beams in a solid space-periodic target," LBL31694 (1991).

P. Eberhard and S. Chattopadhyay, "Asymmetric phi factories - a proposed experiment and its technical feasibility," 15 th International Conference on High Energy Accelerators (Hamburg, Germany, 1992); LBL-32250 (1992).

E. Forest and K. Hirata, "A contemporary guide to beam dynamics," LBL-32793 (1992); also published by KEK (1992).

E. Forest and K. Ohmi, "Symplectic integration for complex wigglers," LBL-32792 (1992); also published by KEK (1992).

M. Furman, "Beam-beam issues in asymmetric colliders," invited talk, in Procedings of the International Conference on B Factories: The State of the Art in
Accelerators, Detectors, and Physics (Stanford, CA, 1992); LBL-32561 (1992); invited talk, Spring Meeting of the American Physical Society (Washington, D.C., 1992); L.BL-31752a (1992)

M. Furman, "Beam-beam effects in asymmetric colliders," abstract for the Spring Meeting of the American Physical Society (Washington, D.C., 1992).

M. Furman, "Proposal for experiments at Tristan on beam-beam issues for future Bfactories," LBL PUB-5332 (1992).

$$
\text { , }
$$

M. Furman, Y.-H. Chin, and J. Eden (LBL), J. Tennyson and V. Ziemann (SLAC), and W. Kozanecki (CEN-Saclay and SLAC), "Beam-beam diagnostic from close orbit distortion," 15th International High Energy Accelerator Conference (Hamburg, Germany, 1992); LBL-31888 (1992); Stanford Linear Accelerator Center SLAC-PUB-5472 (1992).

M. Furman, Y.H. Chin, and J. Eden (LBL), W. Kozanecki (DAPNIA/SPP), and J. Tennyson and $\mathrm{V}$. Zic mann (SLAC), "Closed orbit distortion and the beam-beam interaction," preliminary draft; LBL-32435 (1992).

D. Goldberg and G. Lambertson, "Dynamic devices a primer on pickup? nd kickers," chapter in AIP Conference I roceedings Series: Physics of Particle Accelerators (1992); LBL-31664, (1991).

R. Gough, editor, "An infrared free-electron laser for the Chemical Dynamics Research Laboratory: design report," LBL PUB-5335 (1992).

R. Govil, R.A. Rimmer, and A. Sessler ( $L B L)$ and $H$. Kirk (BNL), "Design of $r$ conditioner cavities," 14th International Free Electron Laser Conference (Kobe, Japan, 1992); LBL-32232 (1992).

A. Jackson, "Ideas for future synchrotron light sources," European Particle Accelerator Conference (Berlin, Germany, 1992); LBL-31172 (1992).

J. Johnson, J. M. Byrd, G. Lambertson, and F. Voelker, "Progress on PEP-II multibunch feedback kickers," in Proceedings of the International Conference on B Factories: The State of the Art in Accelerators, Detectors, and Physics (Stanford, CA, 1992). 


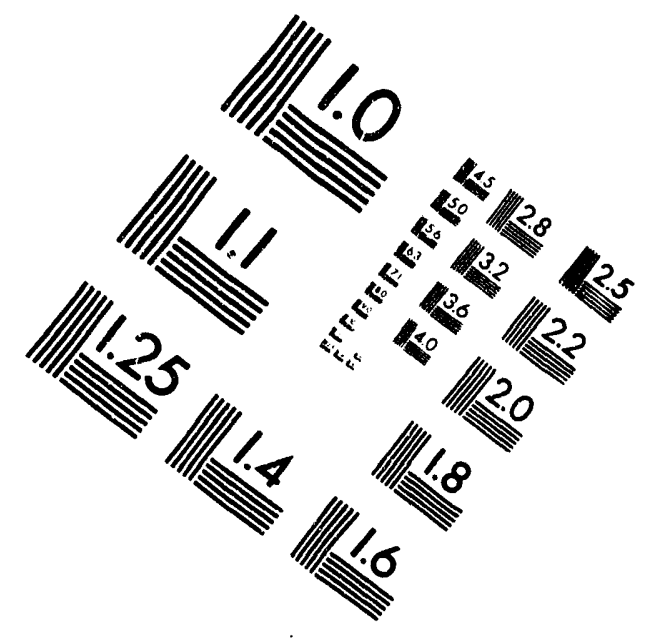

\section{Centimeter}

$\begin{array}{llllllllllllllll}1 & 2 & 3 & 4 & 5 & 6 & 7 & 8 & 9 & 10 & 11 & 12 & 13 & 14 & 15 & \mathrm{~mm}\end{array}$

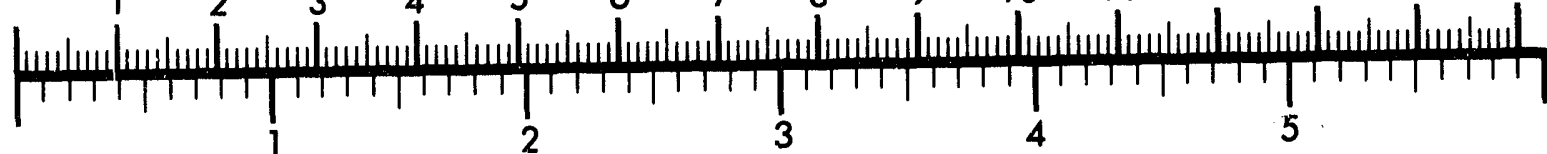
Inches
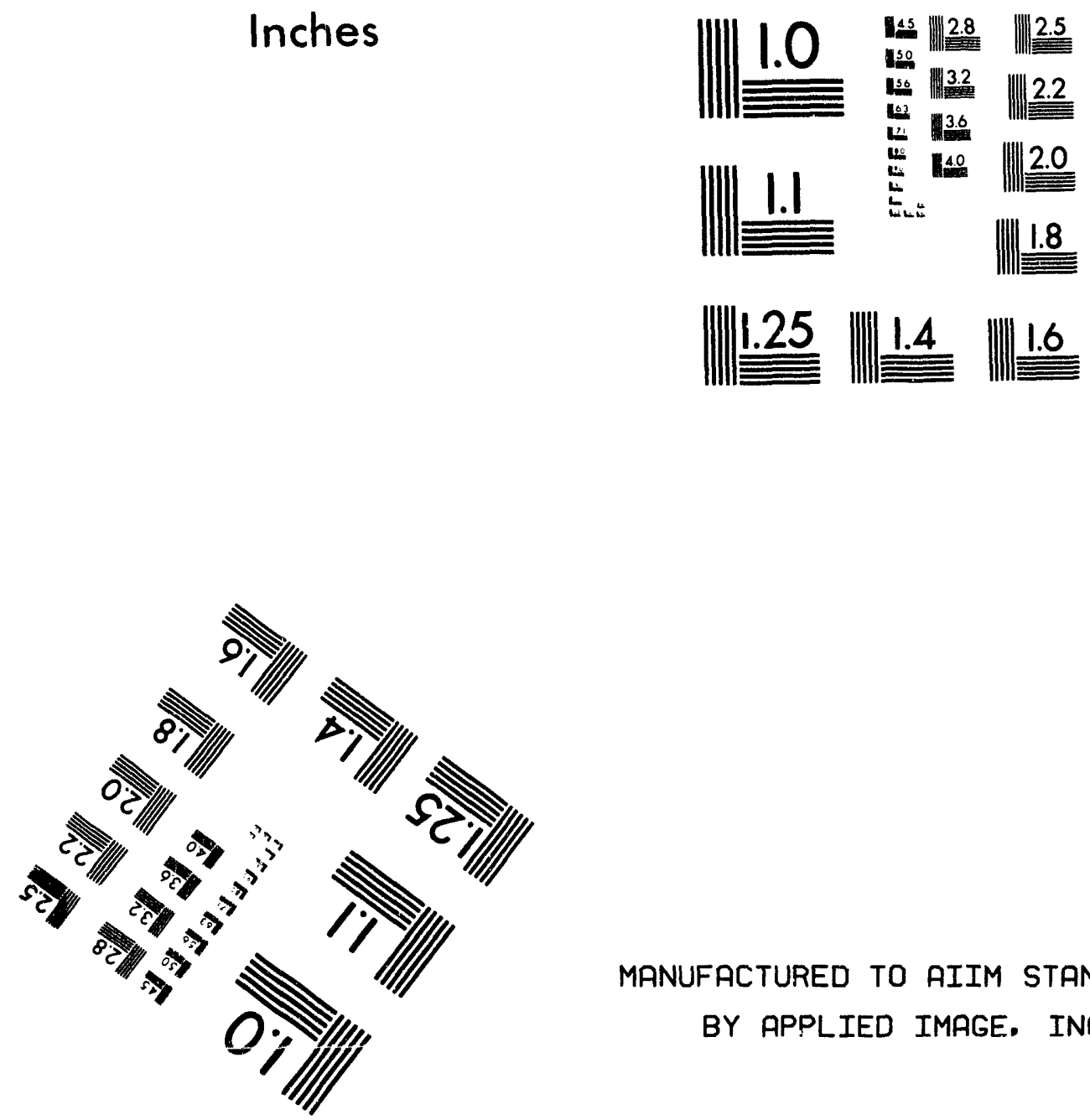

MANUFACTURED TO AIIM STANDARDS BY APPLIED IMAGE. INC.

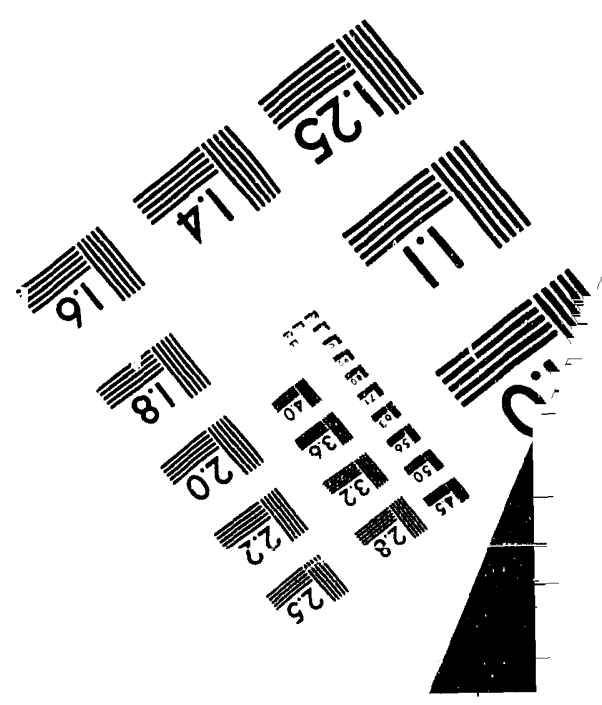



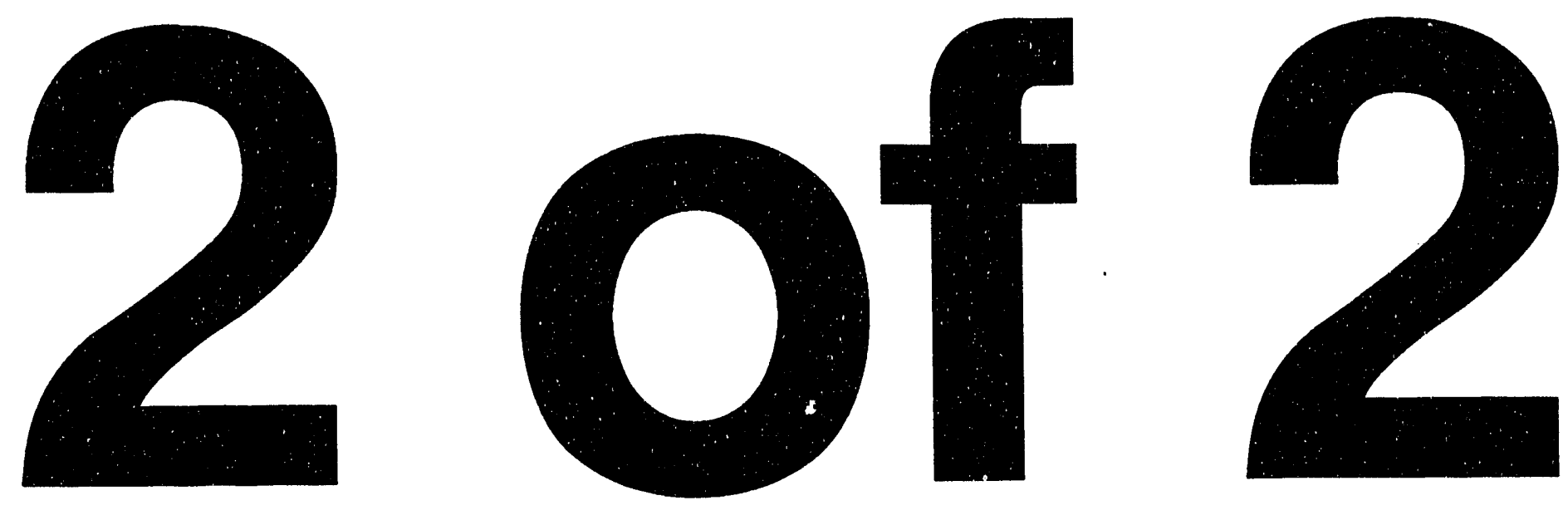


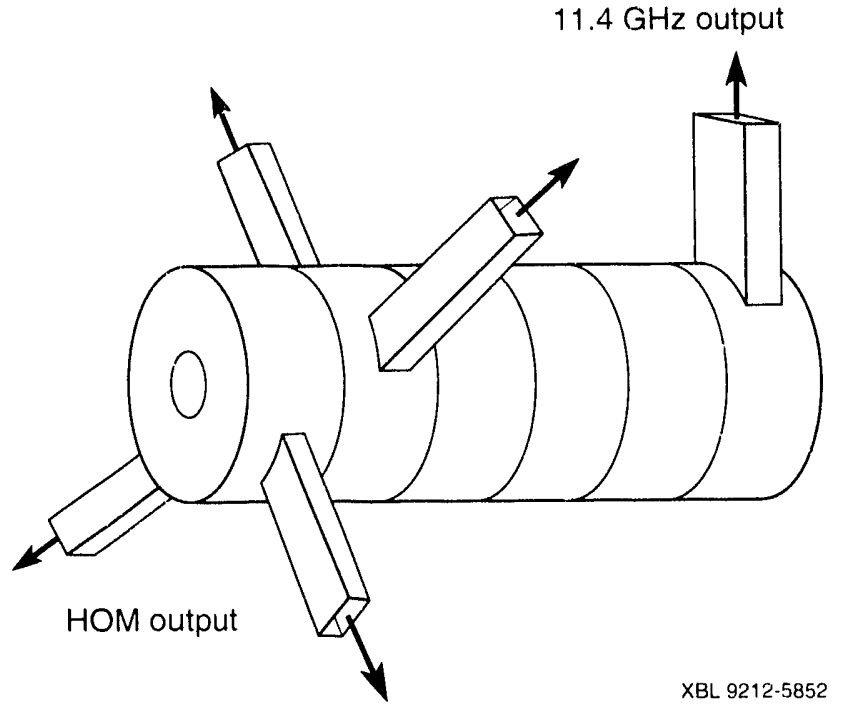

Figure 4-10. When the choppertron was equipped with this structure, a highly disruptive higher-order mode was effectively damped, enabling a 30-ns pulse of $11.4 \mathrm{GHz}$ microwaves at 120 MW. The low phase jitter (about $\pm 2^{\circ}$ ) indicates good frequency stability. The next step is to improve the efficiency of the damper so that the power level in the fundamental mode returns to the hundreds-of-megawatts level achieved (in much briefer pulses) without the damper. (After LLNL illustrations)
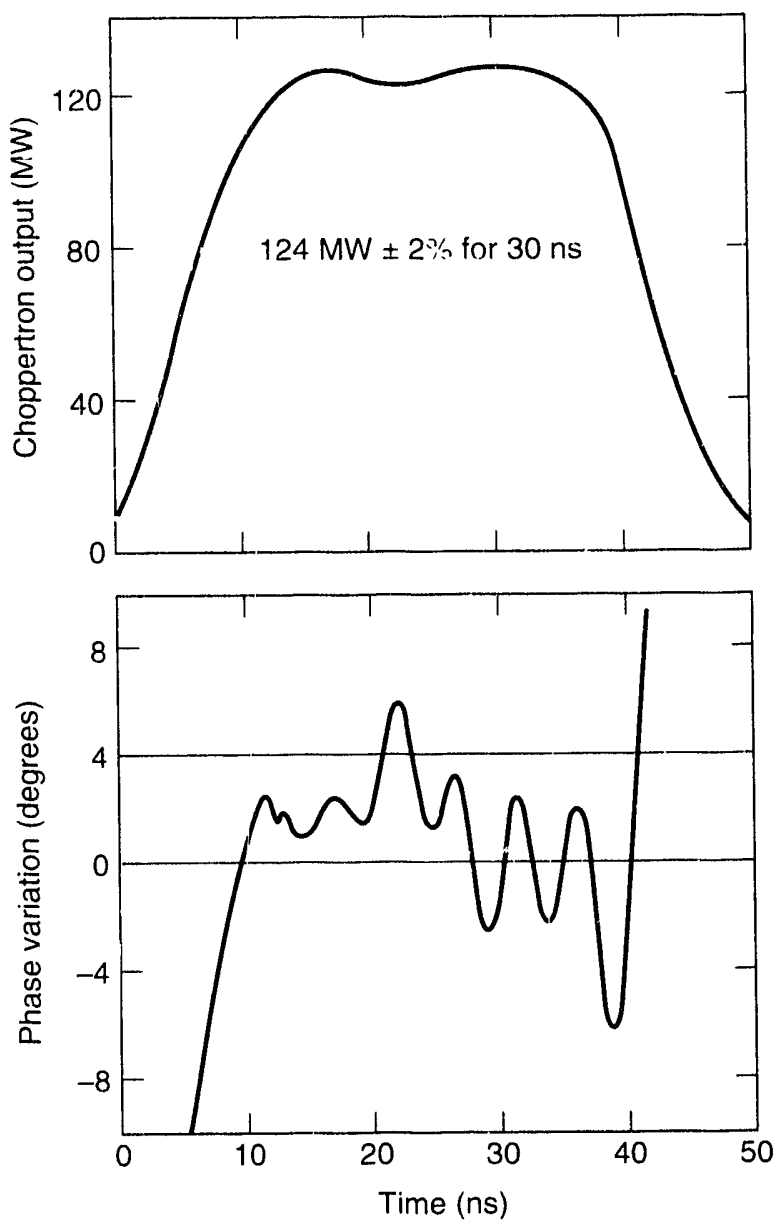

XBL $9212-5852$
Standing-Wave FEL

Horizons for the TBA
The FEL, explored in our original TBA research, remains a proven candidate with great potential. We are developing an idea, called the "standing-wave FEL," in which the radiation is trapped in a standing-wave rf cavity and beat-coupled to a nearby high-gradient acceleration structure. This concept is currently undergoing intense analysis and theoretical study, with no experimental program anticipated in the near term.

The work done on the TBA since we conceived of it 10 years ago has validated the basic concept and the use of either an RK or an FEL as the source of rf power. However, there remains a vast amount of research and development before the TBA can be used in high-energy physics. Here are some of the planned near-term investigations.

- Re-acceleration of the "spent" drive beam (useful for economic reasons) will be examined in a planned 1993 experiment at LLNL.

- There is much theoretical and experimental work to be done in extraction of microwaves from the power source. A demonstration of repeated extraction is being studied at LLNL. 
- Sensitivity studies to determine the importance of various parameters will be important. A great deal of theoretical work has been done; coming years will see more studies on real apparatus.

- Economic matters will be significant in the eventual decision on whether to build a full-scale TBA and in the technological choices within such a project. We are working with LLNL on these issues.

A collaboration with KEK is under way, using an FEL from which up to 30 MW can be extracted at $8.6 \mathrm{GHz}$.

The gain of a free-electron laser or other resonant electron-beam device is limited by the spread in longitudinal velocity and, hence, the energy spread and emittance of a three-dimensional beam. The clectron-beam emittance must be less than the wavelength of the radiation from the device divided by 4. In practice, the energy spread of the beam is often small, so the beam could be "conditioned" with special rf cavities. These cavities would impart more acceleration to the particles traveling longer paths, reducing the spread in longitudinal velocity. We have analyzed this idea with a simple numerical model for beam transport, assuming ideal rf cavities. We have also analyzed an FEL to evaluate its performance with reduced axial-velocity spread; these studies lead us to expect distinct improvements from beam conditioning. During 1992 we computationally analyzed a cavity geometry that promises to greatly increase the beam-cavity coupling. Experiments to test the feasibility of a beam-conditioning cavity are being planned for the Accelerator Test Facility at Brookhaven National Laboratory.

W. Barletta (LLNL), A. Sessler, and L. Yu, "Physically transparent formulation of a freeelectron laser in the linear gain regime," 14th International Free Electron Laser Conference (Kobe, Japan, 1992); LBL-32221 (1992).

W. Barry, J. Edighoffer, and S. Chattopadhyay (LBL) and S. Fornaca (TRW), "Field profile and loading measurements on higher order modes in a two cell $500 \mathrm{MHz}$ superconducting structure," 16 th International Linac Conference (Ottawa, Ontario, Canada, 1992); LBL-32178a (1992).

V.G. Baryshevsky and I. Dubovskaya, "Diffraction phenomena in spontaneous and stimulated radiation by relativistic particles in crystals (review)," LBL-31695 (1991).

1. Ben-Zvi, L.-H. Yu, R. Govil, and A.M. Sessler, "A proposed experiment for beam conditioning," 14th International Free Electron Laser Conference (Kobe, Japan, 1992); Brookhaven National Laboratory report BNL-47857 (1992).
S. Chattopadhyay and K.-J. Kin, "Generation of sub-picosecond x-rays," Workshop on the 4th Generation Light Sources (Stanford, CA, 1992); LbL-31968 (1992).

Y.-H. Chin, "Beam-beam dynamics during the injection process at an asymmetic ring collider," abstract for the Spring Meeting of the American Physical Society (Washington, D.C., 1992); Lawrence Berkeley Laboratory report LBL-31754a (1992).

Y.H. Chin, "Effects of parasitic beam-beam interaction during the injection process at the PEP-II B factory," in Proceedings of the International Conference on B Factories: The State of the Art in Accelerators, Detectors, and Physics (Stanford, CA, 1992); LBL-32468 (1992).

S. Chattopadhyay, K.-J. Kim, R. Byrns, R. Donahue, J. Edighoffer, R. Ciough, E. Hoyer, W. Leemans, J. Staples, B. Taylor, and $M$. Xie, "Design of a superconducting linear accelerator for an infrared free electron
Beam Conditioning

\section{Publications and Presentations}


laser of the proposed chemical dymamics research laboratory at L.BL," IG,th International Linac Conference (Ottawa, Ontario, Canada, 1992); L.BL-32182a (1992).

Y-H. Chin, "Simple formulace for the optimization of the FEL gain length as a function of emittance, betatron wavelength and energy sprend," 14th International Frec Electron Laser Confurence, (Kobe, Japan, 1992); LBL-32249 (1992).

Y.H. Chin, K.-J. Kim, and M. Xie, "Calculation of 3-D) free clectron laser gain: comparison with simulation and generalization to elliptical cross section," $14 \mathrm{th}$ Free Electron Laser Conterence (Kobe, Japan, 1942); LBL-32286 (1992).

Y.H. Chin, K.-J. Kim, and M. Xie, "Threedimensional free electron laser theory including betatron oscillations," submitted to Phys. Rev. A; LBL-32329 (1992).

I.N. Corlett and J.A. Clarke, "New injection kicker magnets for the Daresbury SRS," in Prociedings of the 3rd European Particle Accelerator Conference (Berlin, Cermany, 1992), pp. 1469-1471.

1. Dorfan and A. Hutton (SLAC), M. Zisman (LBL), and W. Barletta (LLNL), "PEP II asymmetric B-factory: R\&D results," 3rd European Particle Accelerator Conference (Berlin, Germany, 1992); LBL-32098a (1992).

I. Dubovskaya, "Coherent generation of visible radiation by relativistic electron beams in a solid space-periodic target," LBL. 31694 (1991).

P. Eberhard and S. Chattopadhyay, "Asymmetric phi factories - a proposed experiment and its technical feasibility," 15 th International Conference on High Energy Accelerators (Hamburg, Germany, 1992); LBBL-32250 (1992).

E. Forest and K. Hirata, "A contemporary guide to beam dynamics," LBL-32793 (1992): also published by KEK (1992)

E. Forest and K. Ohmi, "Symplectic integration for complex wigglers," L.BL-32792 (1992); also published by KEK (1992).

M. Furman, "Beam-beam issues in asymmetric colliders," invited talk, in Procedings of the International Conference on B ratories: The State of the Art in
Acceleraturs, Detectors, and Phesics (Slanford, CA, 1492); 1.BL-32561 (1492); invited talk, Spring Meeting of the American Physical Society (Washington, D.C., 1992); 1.BL-31752a (1942).

M. Furman, "Beam-heam effects in asvmmetric colliders," abstract for the Spring Meeting of the American l'hysical Society (Washington, D.C., 1992).

M. Furman, "Proposal for experiments at Tristan on beam-beam issues for future Biactories," LBBI. I'UB-5332 (1992).

M. Furman, Y.-H. Chin, and ]. Fden (LBL), J. Tennyson and $V$. Ziemann (SLAC), and W. Kozanecki (CEN-Saday and SLAC), "Beam-beam diagnostic from close orbit distortion," 15th International High Energy Accelerator Conference (Hamburg, Germany, 1992); LBL-31888 (1992); Stanford Linear Accelerator Center SLAC-I'UB-5472 (1992).

M. Furman, Y.H. Chin, and ). Eden (LBIL), W. Kozanecki (DAPNIA/SPP), and J. Tennyson and $V$. Ziemann (SLAC), "Closed orbit distortion and the beam-beam interaction," preliminary draft; LBL-324.35 (1992).

D. Coldberg and G. Lambertson, "Dynamic devices a primer on pickups and kickers," chapter in AIP Conference Proceedings Series: Physics of Particle Accelerators (1992); LBL-31664, (1991).

R. Gough, editor, "An infrared free-electron laser for the Chemical Dynamics Research Laboratory: design report," L.BL. PUB-5335 (1992).

R. Govil, R.A. Rimmer, and A. Sessler (LBL) and $H$. Kirk (BNL), "Design of $r f$ conditioner cavities," 14 th International Free Electron Laser Conference (Kobe, Japan, 1992); LBL-322.32 (1992).

A. Jackson, "ldeas for future synchrotron light sources," European Particle Accelerator Conference (Berlin, Cermany, 1992): LBL-31172 (1992)

J. Johnson, J. M. Byrd, G. Lambertson, and F. Voelker, "Progress on P'EP-Il multibunch feedback kickers," in Procertings of the International Conference on B Factories: The State of the Art in Accelerators, Detectors, and Physics (Stanford, CA, 1992). 
K.-. Kim, "Calculation of peak power for short wavelength FFLs," Workshop on the th Gencration Light Sources (Stanford, CA, $1992) ; 1 . B 1-31969(1992)$.

K-l. Kim, "Symmetric and asymmetric multiperiod wigglers in long wavelength limit," submitted to Nucl. Instrum. Meth.; I.BI-.31249 (1991).

K-1. Kim and M. Xie, "Colculation of selfamplified spontaneous emissiom in the short wavelongth region," 1tth Free Electron Laser Conference (Kobe, Japan, 1942); LBI_-32288 (1992).

K.-J. Kim, R. Byrns, S. Chattopadhyay, R. Donalue, I. Edighoffer, R. Ciough, E. Hoyer, W. Leemans, J. Staples, B. Taylor, and $M$. Xie, "An infrared free electron laser system for the proposed Chemical Dynamics Research Laboratory at LBL. based on a 500 $\mathrm{MHZ}$ superconducting linac," 14th Free Electron Laser Conference (Kobe, Japan, 1992); L.BL-32287 (1992).

S. Krishnagopal, "Coherent beam-beam simulations," abstract for the Spring Meeting of the American Physical Society

(Washington, D.C., 1492); I.BL-31747a (1492).

S. Krishnagopal and R.H. Siemann, "An investigation of coherent yuadrupole beambeam effects," 15th International Conference on High Energy Accelerators (Hamburg, Germany, 1992); LBL-32581 (1992).

S. Krishnagopal and R. Siemann, "Some aspects of the two beam performance of DCI," submitted to Nucl. Instrum. Meth.; Stanford Linear Accelerator Center SLACPUB-5653 (1991); LBL-31227 (1991).

S. Krishnagopal and Y.-H. Chin, "Benchmarking beam-beam simulations using coherent quadrupole effects," in Procetdings of the International Conference on $B$ Factories: The State of the Art in Accelerators, Detectors, and Physics (Stanford, CA, 1992); LBL-32516 (1992).

S. Krishnagopal, G. Rangarajan, and A. Sessler, "The multi-cavity free-electron laser," 1 th International Free Electron Laser Conference (Kobe, Japan, 1492); LBLL-32220) (1992).

S. Krishnagopal, M. Xice, and K.-J. Kim, "Suppression of mode-beating in a saturated hole-coupling FEL oscillator," 14 th International Free Electron Laser Conference (Kobe, Japan, 1992); L.BL-32223 (1992).
C. Lambertson, "Transierese fledback in a 100 TeV storage ring," in Precedetines of the 19th Workshop on Maximizing L uminosity of Hadron Colliders at 100 TeV' If rice, Itals, (4)1); 1.131.-325.37 (1492).

W. Leemans, I. Edighoffer, K.-l. Kim, and S. Chattopadhyay, "Novel techniques for single-pulse spectrum and pulsewidth mensurements for an IR-FEL," 1 th Free Electron Laser Conference (kobe, lapan, (0)2); LBL-3228+ (1092).

W. Leemans, J. Edighoffer, M. Xic, K.-J. Kim, and S. Challopadhyay, "Bench-testing of IRFEL hole-coupled resomator designs using a $C W$ - HeNe laser: mode profile and outcoupling efficiency," 1t th Free Electron Laser Conference (Kobe, Japan, 1992); LBL32285 (10)2).

C. Rangarajan and A. Sessler, "Sensitivity studies of a standing-wiave free-electron laser," Third Workshop on Adranced Accelerators (Port lefferson, NY, 1992); LBL$32+6.3(1992)$

R. Rimmer, D. Goldberg, C. Lambertson, and F. Voelker (LBL.), K. Ko, N. Kroll, R. Pendelton, and $H$. Schwary (SLAC), and $N$. $F$. Adams and $M$. de Jong (AECL), "Higher order mode damping studies on the PEI'-1I Bfactory RF cavity," 3rd European Particle Accelerator Conference, (Berlin, Cermany, 1992); L.BL-32549 (1992).

L. Schachinger, "Summary of the working group on modeiling and simulation," presented at the Fifth Advanced ICFA Beam Dynamics Workshop (Corpus Christi, TX, 1991); LBL-31665 (1991).

A.M. Sessler, "Linac-based VUV FEL_s- a review," abstract for the Spring Meeting of the American Physical Society (Washington, D.C., 1992); LBI-31696a (1992).

K. Takayama, R. Govil, and A. Sessler, "Macroparticle theory of a standing wave free-electron laser two-beam accelerator," submitted to Nucl. Instrum. Meth. A; LBL$32(44+(1942)$

C. Wang, "Conditioner for a helically transported electron beam," Workshop on Advanced Accelerator Concepts (Port Jefferson, NY, 1992); LBL-32222 (1992).

J. S. Wurtele, D.H. Whittum, and A. M. Sessler, "Common analysis of the relativistic klystron and the standing-wave free-electrom 
laser two-beam accelerater," 15th

Intermational Conference on High Energy Accelerators (Hamburg, Germany, l(4)2); L.BL- -32580 (I)(4) ).

1. Wurtele, D. Whittum, and A. Sessler, "Impedance-based analysis of the relativistic klystron and the coupled-cavity free-electron laser two-beam accelerator," Third Workshop on Advanced Accelerators (P'ort Jefferson, NY, 1992); LBI . 31848 (1992)

M. Xie, "Variational analysis of 3-D FEL gain in beam distribution approach," 14th Free Electron Lalser Conference (Kobe, Japan, 1942); I.BL-3229) (1942).
M. Zisman, "B factory rf system design issues," in Procidedings of the Intermational Conference on B Factories: The State of the Art in Accelerator's, Detectors, and l'hysics (Stanford, CA, 1992); L.BL-324.46 (1992).

M. Zisman (LBBL), R. Bell, J. Dorfan, and 11 . Schwar\% (SI.AC), and W. Barletta and $M$. Calderson (I.LNL), "The PEP-II asymmetric B factory: design update and R\&D results," 15th international Conference on High Energy Accelerators (Hamburg, Germany, 1942); LBL-32559 (1942); Stanford Linear Accelerator Center SI.AC PUB-5852 (1992); Lawrence Livermore National Laboratory UCRL-JC-111380() (1992). 


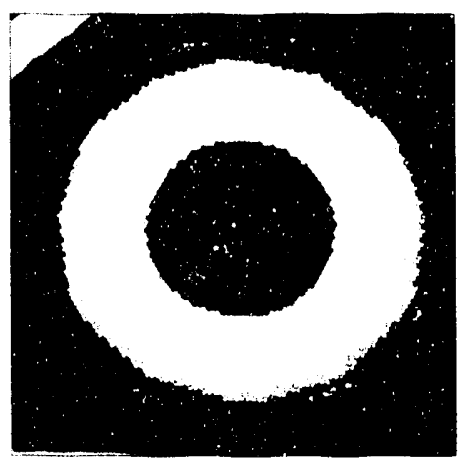

5.

\section{SUPERCONDUCTING MAGNETS}

\footnotetext{
T
}

HOUGH PERHAPS MOST FAMILIAR IN ACCELERATORS such as the Superconducting Super Collider (SSC), superconducting materials are currently or potentially useful in many other fields, ranging from magneticresonance imaging to magnetically levitated trains. The Superconducting Magnet Program in AFRD takes an integrated approach to these magnets, with involvement at all phases from basic development of superconducting materials to evaluation of finished magnets.

One of our most significant 1992 accomplishments was the completion of the SSC Magnet Industrialization Program, in which industry representatives worked alongside LBL engineers and technicians to learn how to build the technically challenging collider quadrupole magnets. This degree of technology-transfer activity is inherent in the program's operations, which rely at all levels upon two-way interaction with industry.

The SSC work largely culminated in 1992 with the exhaustive (and successful) testing of six full-length collider quadrupoles. As this effort diminished, the program's emphasis shifted toward other areas: generic R\&D that will benefit future accelerators, for instance, as well as nonaccelerator applications of superconducting magnets and basic investigations of materials.

\begin{tabular}{|c|c|c|c|}
\hline C. Taylor (program he'ut) & W. Gilhere' & A. lekedist' & Administrative Support \\
\hline I. Amerman* & M.A. Cireen* & F. l'erry* & S. P'ereira \\
\hline B. Ardenyi $\mathrm{i}^{*}$ & M.1. Gireent & 1. Rechen ${ }^{* *}$ & I.A. Zelver \\
\hline R. Armer** & L. Hawrystor' & 1. Rementurch ${ }^{*}$ & \\
\hline P. Baralle* & M. $\mid \mathrm{c} / \mathrm{m}^{*}$ & K. Rencher' & Students \\
\hline B. Benjamine* & II. Higley* & 1. Royet* & C. Brown \\
\hline R. Benfegerdes & E. Hiss" & C. Saunters"t & K. Etter \\
\hline B. Bensiek* & 1. Hern & R. Scanlan & S. Eylon \\
\hline ['. Bish" & R. L.atever* & D. Schafer** & E. Kornblum \\
\hline J. $B\left(x \cdot h m^{*}\right.$ & 1. Laslett ${ }^{* *}$ & 1. Sopher" & P' McManaman \\
\hline V. Bradt ${ }^{* *}$ & $X .1 . i$ & D. Thewlis" & M. Sivta \\
\hline S. Caspi* & A. lieloke & N. Twigg" & H. Tisui \\
\hline 1. Cortella* & R. Méuserer* & D. Van Dyke' & C. Wang \\
\hline S. Dardin* & K. Mirk** & H. Van Oort & N. Wilco: \\
\hline D. Dell'Oreo* & 1. O'Neill* & A. Wandestorde* & I. Wong \\
\hline D. Dietterich & R. Oort* & J.S. Zelver* & \\
\hline B. Chiorsut* & & & \\
\hline
\end{tabular}

* Enginerering Division

** Relired

* Babouch \& Wilcor

'SSC Loboraturs

${ }^{1+}$ Contract 
Among the highlights was a field-strength record for accelerator-type magnets: just over $10 \mathrm{~T}$ in the magnet D19, made with niobium-titanium superconductor.

The 10-T regime, however, appears to be near the practical limit for niobium-titanium. In the push toward $13 \mathrm{~T}$ and beyond in accelerator magnets, we are exploring other materials that are not currently as well-proven and economical, but hold the promise of higher fields. They include brittle materials such as niobium-tin, as well as "high-temperature" bismuth-oxide superconductors operated at liquid-helium temperatures.

Innovative approaches are also being taken in ancillary fields. A new method that uses fiber optics to measure strain through optical interferometry is being investigated. Also being developed is an analytical approach that better integrates magnet design and accelerator physics.

The SSC. He largest scientific instrument man luns e'er attempted to build, e'ntails many technical challenges. Some of the most significant challenges arise from the supercontucting magnets. The magnets have to meet exacting specifications for precision and durability. Furthermore, the most-used magnet type's, especially those' in the main collider rings, must lend the'nselenes to industrial mass production; the pair of 52-mile-circumference collider rings will need more than 10000 magnets, mostly dipoles and ounatrupoles.

Our program at LBL has contributed to both of these types of magnets. In 1989, weresumed work on the quadrupole (focusing) magnet, which hat begun in 1987 but had been suspended so that we could concentrate on the dipole. Because the fullscale quadrupoles are only $5 \mathrm{~m}$ long (in contrast to the 17-m dipoles), it uns feasible to conduct the entire effort in the LBL facilities, including fabrication and testing of prototypes* and subsequent transfer of the technology to industry.

By the end of 1992, we had exhaustively tested all six full-length quadrupole prototypes. The fabrication and testing effort often indolved the close cooperation of engineers and technicians from Babcock and Wilcox and from Siemens under the auspices of the Magnet Industrialization Program. They worked alongside their LBL counterparts, beginning in August 1991, to learn how to build and test these magnets.

The Magnet Industrialization Program resulted in the transfer of both knowehow and mamufacturing technology to the Babcock and Wilcox team, which has contracted with the SSC Laboratory to design, build, and test 1-m working models and 5-m prototypes, thereby positioning themselves to bid on mass production later in the SSC project. Our SSC collider-quad rupole effort, except for some probable ongoing consultation and assistance, was thereby successfully concluded.

The results of "training" the six 5-m prototypes are shown in Figure 5-1. The last five fully met the SSC's specification for quadrupole training behavior, which requires that the magnet exceed the operating current of $6560 \mathrm{~A}$ by $5 \%$

\section{SSC Magnet Industrialization}

Collider Quadrupole
Development

* At first, we worked with 1-m functional "models" of the SSC quadrupole as well as the 5-m prototypes. The $1-\mathrm{m}$ models, which are quicker and cheaper to make, were useful for many of our research activities. The Babcock and Wilcox effort will also begin with 1-m models. Our base program of non-SSC magnet research is performed mainly with short dipoles. 


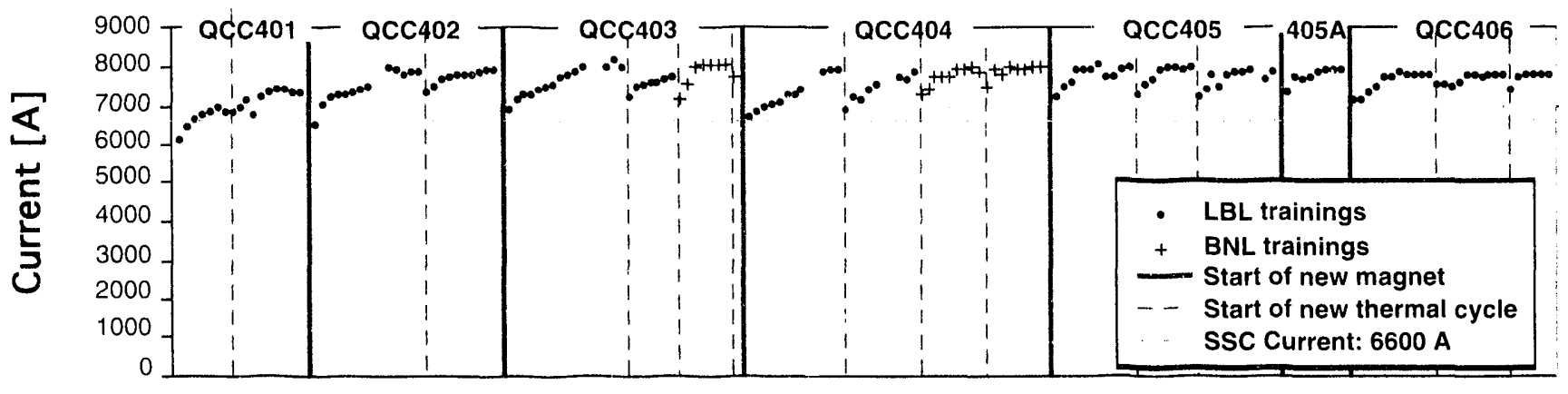

Figure 5-1. Training results for full-scale SSC collider quadrupole prototypes show that the desired field (horizontal dashed line) was usually achieved on the second quench or sooner, with subsequent performance well in excess of requirements. QCC 401 was the first "throwaway" prototype; QCC 405 examined a modified yoke. The vertical dashed lines indicate experiment interruptions during which the magnet was brought back up to room temperature.

after no more than three quenches and subsequently reach the operating current without quenching. They achieved $6560 \mathrm{~A}$, corresponding to a field gradient of $211 \mathrm{~T} / \mathrm{m}$ across the $4-\mathrm{cm}$ bore, ${ }^{*}$ on or before the second quench. After a modest amount of training, they far exceeded the design requirements.

Figure 5-2 shows successive stages in the fabrication of one of these magnets. First, the superconducting cable is formed into the proper shape on a mandrel. For short magnets the cable supply spool and tension control remain stationary while the mandrel revolves. In 1989, for making 5-m magnets, we developed the equipment shown here, in which the mandrel remains stationary while the cable spool travels around in a "racetrack" path. The cable and mandrel are inserted in a precisely machined molding cavity where heat is applied. A heat-activated B-stage epoxy on the windings holds them in place until laminated-metal collars can be installed with a hydraulic press. The result is a low-cost yet rigid structure that maintains the coil positions accurately even under the stress of multi-tesla magnetic fields. Finally, iron yokes and a welded stainless-steel jacket are applied.

Each of these magnets is slightly different, for engineering them is an iterative process. Although physicists and engineers understand quite well how to build adequate magnets of this type, a great many potentially beneficial innovations have yet to be tested. (Now that our SSC work has ended, the final design will be determined by Babcock and Wilcox or other industrial companies that will mass-produce the 1664 collider quadrupoles.) Each magnet-a typical completed unit is shown being inserted in its cryostat in Figure 5-3-incorporates some feature that we think will improve performance, reliability, or manufacturability.

An example is QCC 405. In it, we tried out a different design in which the iron yoke is clamped down onto the pack of interlocking collars, hoping that this would improve the magnet's training behavior-an approach that

\footnotetext{
* In the original SSC design, all the principal magnets in the main collider lattice had a $4-\mathrm{cm}$ bore. Later, the dipole bore was changed to $5 \mathrm{~cm}$ to ensure a sufficient transverse "good-field" region. The concerns were not applicable to the much shorter collider quadrupoles, so there the less costly $4-\mathrm{cm}$ design remains in use.
} 

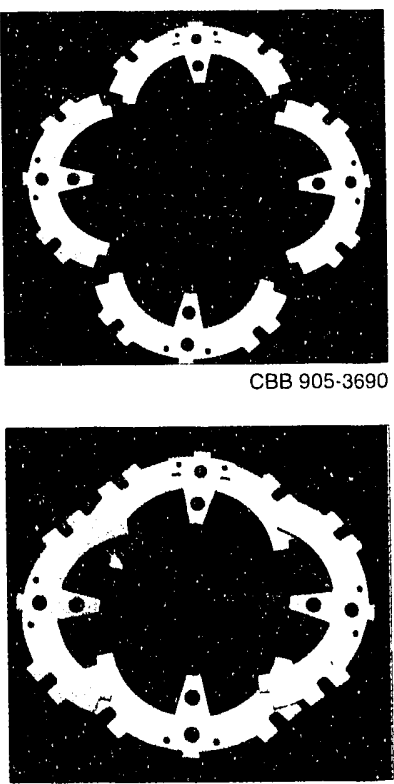

CBB 905-3686

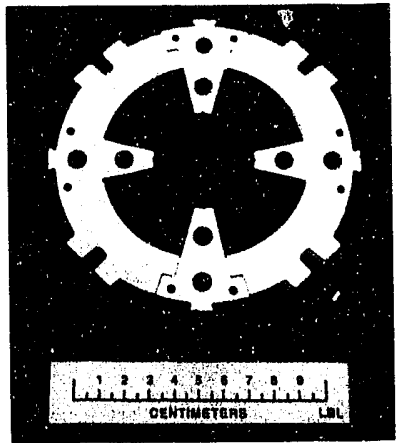

CBB 905-3688

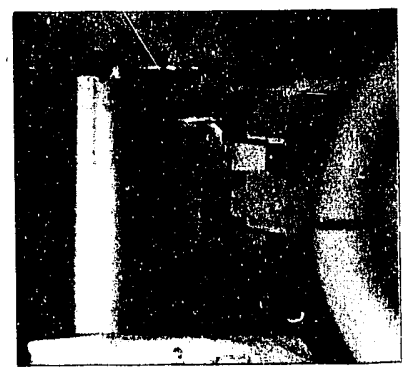

CBB $878-7135$

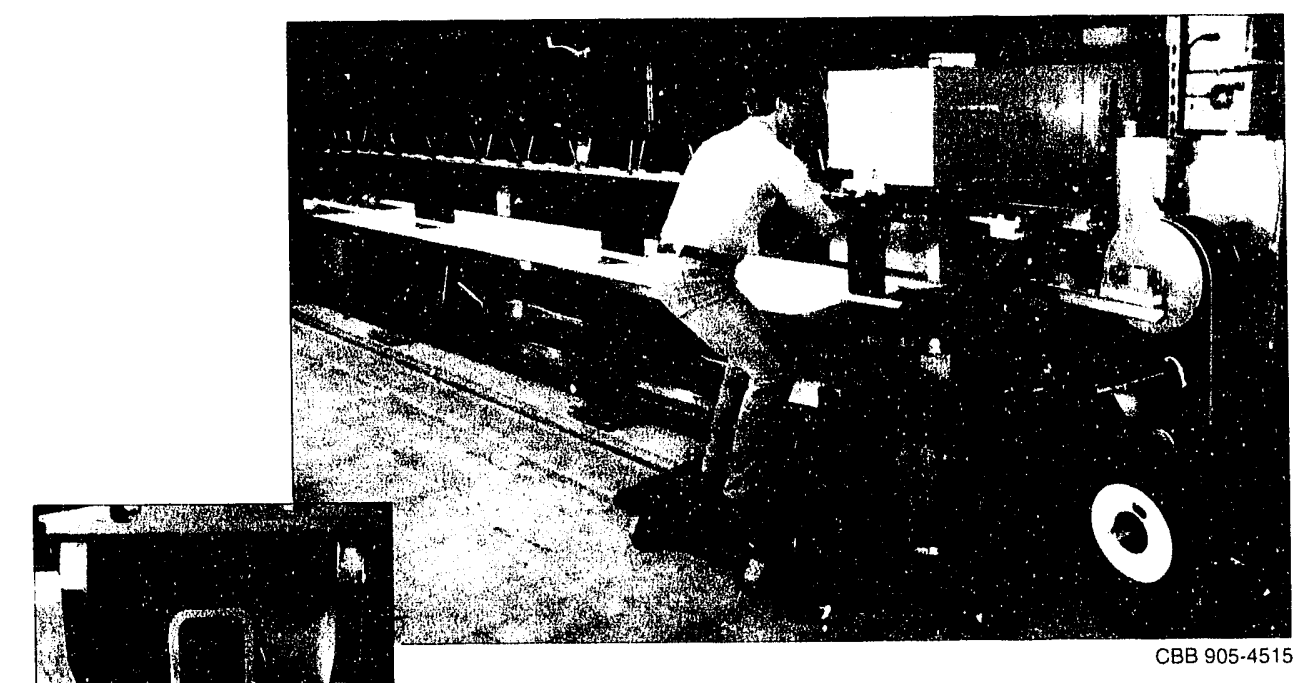

Figure 5-2. Stages in the assembly of a magnet include (counterclockwise from top right) winding the layers of superconducting cable on a mandrel, compressing and heating this assembly inside a precision mold, installing collars, and finally applying and welding the stainless-steel jacket at the outer edge of the cold mass. During magnet collaring, a hydraulic press compresses the collar pack enough for tapered keys to be driven into the slots in the collar as the external pressure is relieved. The collar pack is thus drawn tightly around the coils, resulting in a stable assembly that puts a pressure of several thousand pounds per square inch on the windings. The details at far left show how the parts of the collar pack for a quadrupole fit together.
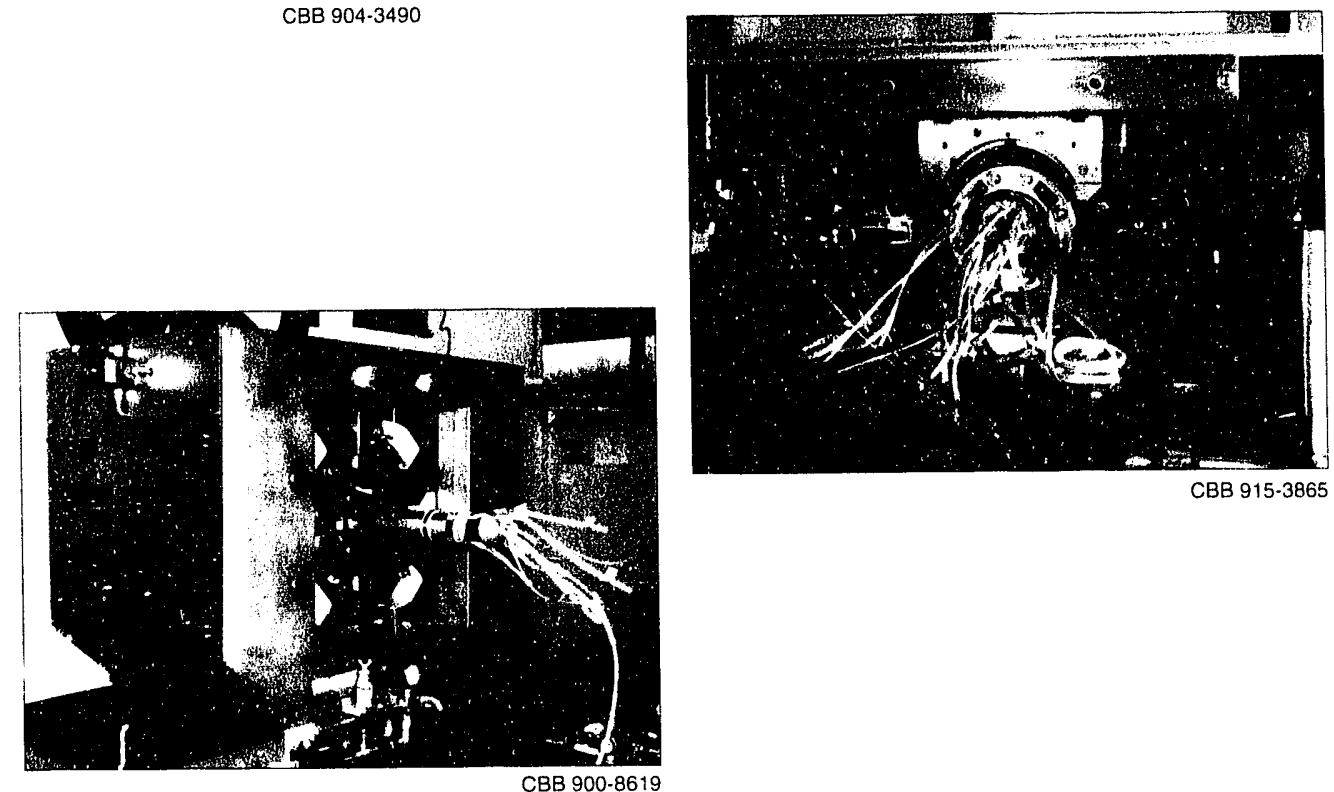
Figure 5-3. A prototype SSC collider quacirupole magnet is inserted in its cryostat for testing.

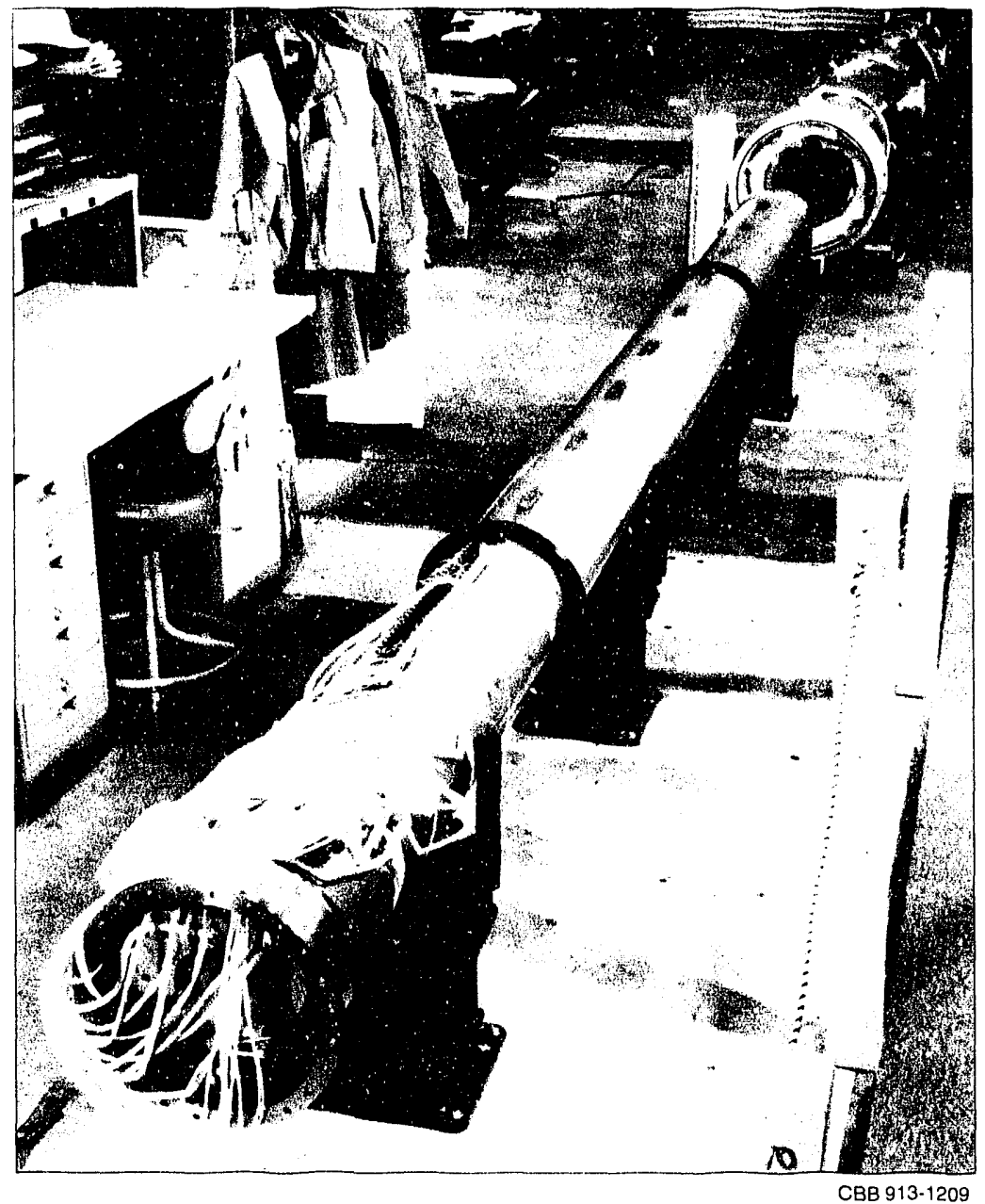

was originally suggested by the SSC Laboratory for the dipoles. However, the training behavior of the inherently more-rigid quadrupole was improved little, if at all. The lack of substantial improvement in the already-acceptable training behavior suggests that the idea will not be applied to the collider quadrupoles.

After a magnet has been fabricated, it is operated in a cryostat at everincreasing current until we detect a "quench," which is a rapid heating and consequent loss of superconductivity. Each unit is equipped with extensive instrumentation, such as load cells to measure the forces developed in the windings and voltage taps to pinpoint the origin of quenches. This information can be used to improve design and fabrication in the search for greater reliability and predictability.

In keeping with the highly applied nature of the SSC program, quality assurance and quality control are carried to great lengths. The emphasis begins in engineering, where we strive to create designs and procedures that reduce the need for skilled craftsmanship and the variability it entails. Precision tooling is another key feature. Coils are wound to a uniformity of \pm 0.001 to 0.0015 inch (azimuthal) over the 5-m length of each magnet, and similar degrees of reproducibility from one magnet to another are sought. Each of the major manufacturing fixtures-the molding, collaring, skinning, 
and yoking presses- -is built to close telerances, and automated, numerically controlled processes are used whenever possible. The result is a linal assembly that is true to within \pm 0.0105 inch in straightness and \pm 11.25 milliradian in twist. Each coil is measured at 20 locations along its length, using a semi-automated, numerically controlled measuring machine.

The uniformity of the magnetic field is also measured, as is its purity, or freedom from undesirable higher-order fields. The magnetic measurements are usually made at room temperature, which is much easior; correlations with measurements made in a cryostat show that room-temperature measurements of these parameters provide a good indication of cryogenic performance.

Measurement and documentation are also important factors in quality. Each magnet is made according to standard procedures and is accompanied by a logbook where some 1000 electrical and mechanical measurements are recorded. This kind of information constitutes a knowledge base of normal readings and critical parameters that will be useful during mass production.

\section{"Breaking In" a Newe Magnet}

Ncarly all of our tests involve training, the process by which a very strong superconducting electromagnet is brought up to its full capability in several steps. The mechanism of training is not definitively understood. The predominant hypothesis centers on small, unavoidable mechanical instabilities in the windings. When the magnet is first energized, the windings, which are themselves affected by the mannetic field, move slightly as they bed in. This motion, although minuscule, is enough to cause frictional heating, and at liquid-helium temperatures even a small amount of heat can make a small part of the winding go from a superonducting state into a normal, resistive state. Then, at high currents, the entire magnet heats up, or "quenches," and the energy has to be removed from it quickly. Measures can be taken to control a quench gracefully and avoid ruining the magnet, but a quench in any of the thousands of magnets in an SSC collider ring would halt operation for several hours while the problem was resolved and the ring was reloaded with accelerated protons.

The need for training can be circumvented, or at least greatly reduced, through a procedure called conditioning, which we demonstrated in 1986. To condition a magnet, we temporarily reduce the temperature below the design value, which enables us to increase the current and therefore operate for a time at a higher magnetic field than the magnet was designed for. This results in considerable overpressure; once a magnet has been conditioned, the remaining quench-causing mechanical instabilities will not be triggered by normal operation. Nonetheless, we continue to work with nonconditioned magnets that must be trained; the training behavior of a magnet offers great insight into design and performance, and such detailed knowledge may point the way to building magnets that give their full performance without either training or conditioning.

In 1988, we learned that very small changes in the coil-support structure can cause significant differences in training behavior. For example, when the collars are removed from a trained magnet and then put back around the same coils in even a slightly different fashion, the magnet must be retrained. Thus we can test the influence of changes on training without having to build completely new magnet parts for each test.

The SSC is planning to use conditioning if necessary. However, the last four of our unconditioned SSC collider quadrupole prototypes reached the field strengths required by the SSC without quenching, as have full-length dipole prototypes built at Brookhaven National Laboratory and Fermilab. 


\section{Advanced Magnets}

D19: A Record-Seiting Magnet

Figure 5-4. This graph shows the training behavio: of dipole D19 on its way to an ultimate field of $10.06 \mathrm{I}$ in the middle of the bore-a record for acceierator-type magnets.
Althengh applied de'clopment and for the SSC dominated aur actionities in recent years, are have continned to ine'stigate other aspects of superconducting-magnet science and tedmology. The fintings will be relewant to accelerators other than the SSC and to superconducting-magnet applications other than accelerators.

These investigations also represent the future of our program as the $S S C$ magnet effort shifts its focus away from laboratories and $R \& D$ toward private industry and mass production. Our current and future directions include advances in high-field magnets; specialty magnets such as interaction-region focusing quadrupoles for "particle factory" colliders; and magnets that are stronger, more reliable, and easier to assemble.

To advance the state of the art in magnets and to support our development of superconducting cables and the machines to make them, we build experimental magnets comparable in size and shape to those actually used in acceleraiors. The aforementioned field-strength record for such magnets of $10.06 \mathrm{~T}$ central field (approximately $10.4 \mathrm{~T}$ near the conductor) was set using D19, a high-field dipole. Figure 5-4 shows the training behavior of D19 en route to $10 \mathrm{~T}$.

D19 uses the same niobium-titanium cable as the SSC dipole magnets: 30) strands in the inner layer of windings, 36 in tise outer layer. The field-record attempts were made at $1.8 \mathrm{~K}$, with a current of about $9.4 \mathrm{kA}$. It reached the

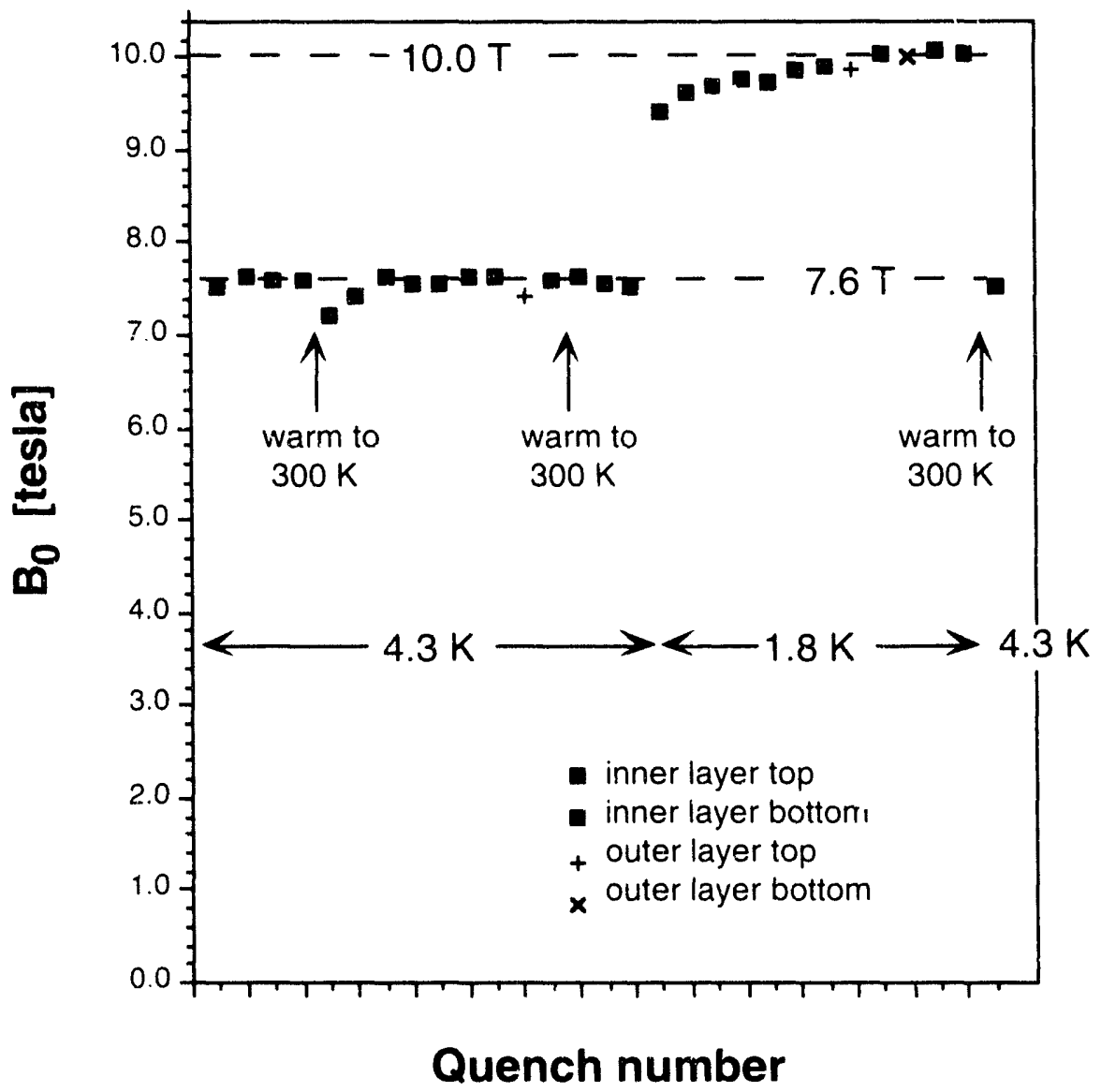


SSC dipole field of $6.6 \mathrm{~T}$ at $5.8 \mathrm{kA}$ and $4.3 \mathrm{~K}$. This is considerably better efficiency than could be obtained from an earlier design such as the SSC collider dipole, which requires $6.6 \mathrm{kA}$, also at $4.3 \mathrm{~K}$, to produce the same field." The cross section in Figure 5-5 reveals the noncircular inner profile of the irsn yoke. This shape, an existing idea not previously applied to accelerator-type magnets, maximizes the magnetic-field contribution of the iron while keeping high-field saturation effects down to reasonable levels.

Currently in the works is D19B, a follow-on project using the same design but incorporating superconductor that has artificial flux-pinning centers for higher critical current.

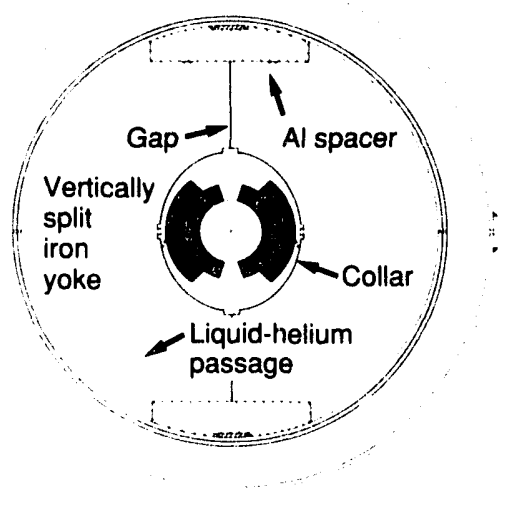

XBL $9112-6875$

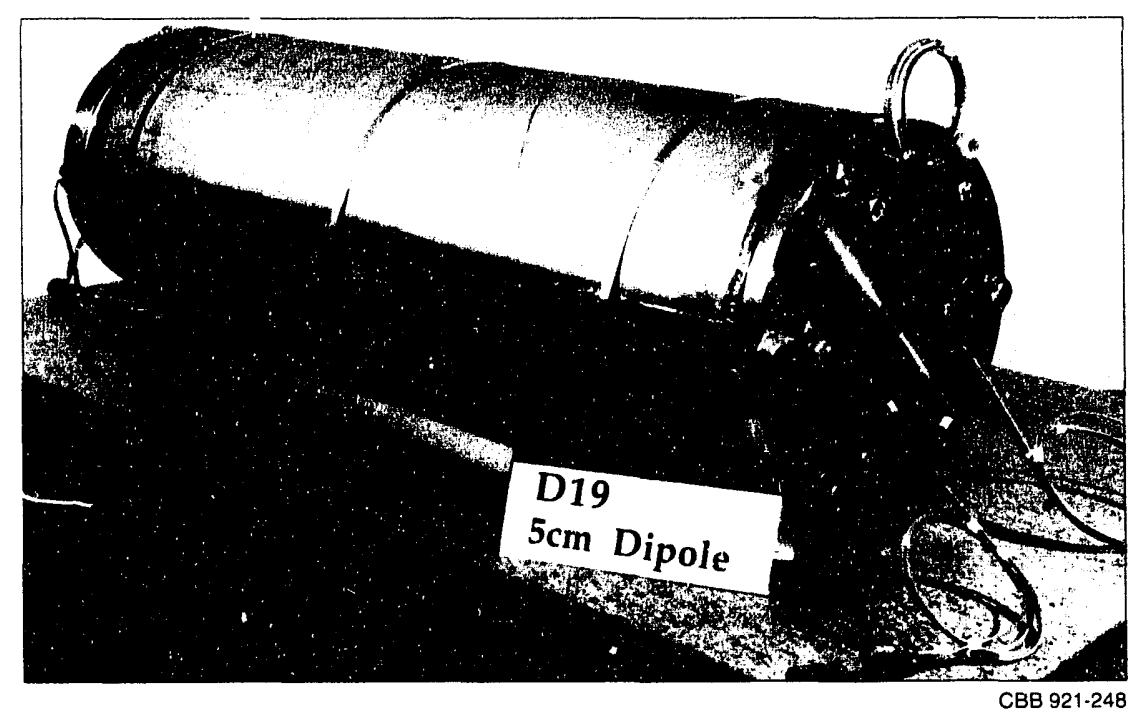

Figure 5-5. Dipole D19, the 10-T magnet, is more efficient than the similar SSC magnets because of the noncircular inner profile of the iron yoke. This shape maximizes the magnetic-field contribution of the iron while keeping high-field saturation effects down to manageable levels. Another interesting feature is the vertically split iron yoke with aluminum-alloy spacers between the halves. The spacers maintain a predetermined gap between the halves at room temperature but allows them to shrink together tightly at cryogenic temperatures. This maintains the high compresive load on the coils and prevents the windings from shrinking faster than the iron yoke during cooldown. Such differential contraction would relieve the compression of the windings and thus undo the "training" process that allows the ultimate magnetic field to be reached.

\footnotetext{
* A large, high-technology apparatus that must be completed in a timely manner, be it an aircraft, supercomputer, or accelerator, connot necessarily incorporate all the latest innovations. As improvements are invented, the advantages they offer must be weighed against schedules and budgets, a rule that has become more and more stringent as the project progresses and components go into mass production.
} 


\section{D20: The Push Beyond}

$10 \mathrm{~T}$

Figure 5-6. With dipole D20, we will use $\mathrm{Nb}_{3} \mathrm{Sn}$ superconductor in the push toward field strengths previously unachievable in accelerator-type magnets. Two layers of superconducting cable47 strands in the outer layers, 37 in the inner layers-will be wound in a double-pancake configuration. The mechanical design is similar to that of D19. However, because $\mathrm{Nb}_{3} \mathrm{Sn}$ is embrittled by the heat treatment that makes it superconducting, we will have to anneal the cable after winding. The goal of D20 is $13 \mathrm{~T}$, representing a short-sample limit for the $\mathrm{Nb}_{3} \mathrm{Sn}$ material of about 13.3 T.
Another advanced magnet, D20, is now being designed. It will press through the 10-T barrier (and hopefully well beyond) by using niobium-tin superconductor. The goal of D20 is a "short-sample" field of about $13 \mathrm{~T}$ at $1.8 \mathrm{~K}$. (The short-sample field represents the maximum performance of the superconducting cable and is determined by measuring the conductivity of a short sample in an external magnetic field. An actual magnet incorporating the cable might, if designed and constructed well, approach the short-sample limit near the edge of the aperture, where the field is strongest.)

The effort to reach beyond $10 \mathrm{~T}$ in accelerator-type magnets presents a number of challenges above and beyond magnetic design. Mechanical stresses become far greater than those in the SSC magnets, for example. This can have a variety of adverse effects. In its most severe form, the strain can distort the magnet. It can also cause the small movements of the cable that lead to quenches, and can reduce the critical current of the superconductor (an effect that we are investigating).

Most importantly, $\mathrm{Nb}_{3} \mathrm{Sn}$ in its finished, superconducting form is much more brittle than the widely used $\mathrm{NbTi}$ and cannot be wound into cable. Instead, cable containing the ingredients is wound onto magnet forms and then heated to $700^{\circ} \mathrm{C}$ to make the ingredients react (which also causes embrittlement). This magnet-making procedure is essentially a new technology, and an important one, since all of the other materials potentially useful for these high-field magnets also require heating after winding. (Ductile "ternary," or three-part, compounds like NbTiTa might offer small improvements over $\mathrm{NbTi}$, but the high-field frontier clearly belongs to brittle superconductors. The brittle compounds, at least theoretically, hold the potential for current densities four or five times greater than that of $\mathrm{NbTi}$.) Therefore the techniques developed for D20 will be widely applicable to future R\&D. D20 is diagrammed in Figure 5-6. Although intended solely as an $\mathrm{Nb}_{3} \mathrm{Sn}$ "trial horse," it has many characteristics generically applicable to future hadron colliders. These characteristics include the high field itself (which would permit higher energies and/or more-compact layouts) and an iron flux-return yoke that could accommodate twin bores. Twin bores might prove more economical than the approach used in the SSC and present-day colliders, in which, variously, two beams rotate in opposite directions through the same pipe or two pipes run through separate lattices of magnets. 
Although our program grew up alongside high-energy physics and is strongly oriented toward service to the accelerator community, there are many other applicatio's for high-field superconducting magnets. One of them is the field of nuclear magnetic resonance (NMR) and magneticresonance imaging (MRI). We are exploring a cooperative project with Wang NMR of Livermore, CA and the Bitter National Magnet Laboratory at the Massachusetts Institute of Technology to develop the conductor for a gigahertz NMR spectrometer. This proton spectrometer would be part of the Molecular Science Research Center, an initiative proposed by Pacific Northwest Laboratory, and would be especially well suited to studying the large molecules and systems of molecules that are of interest to biologists. The spectrometer design requires a large $23.5-\mathrm{T}$ magnet. ${ }^{* *}$

As part of a "vertically integrated" approach to superconducting magnet $R \& D$, we conduct a diverse program of investigations in superconducting materials. Current projects explore artificial pinning center (APC) materials and investigate still-embryonic but promising applications of high-Tc superconductors. Other projects include a novel interferometric method of measuring strain in magnets; it uses optical fiber not in its conventional role as a "light pipe" for imaging, but rather as a physical sensor. We are also embarking upon an innovative approach to the design of magnets that better integrates this process with the methods and conventions used for beam-dynamics calculations by accelerator physicists.

As we look toward the future of superconducting-magnet development, we realize that materials-science research plays a key role in achieving higher, more-uniform, and more-predictable magnetic fields. A promising recent line of inquiry involves the APC concept. iit APC superconductors, the random distribution of pinning centers, which ordinarily arise from precipitation, is replaced with a more-precise distribution that matches the magnetic fluxoid lattice for a given field strength.

A fluxoid is the site of one quantum of magnetic effect and may be thought of as the place where a line of magnetic flux penetrates the superconducting wire. Ordinarily, fluxoids can move through the superconductor in response to an applied magnetic field, and energy is dissipated. In artificial flux pinning, a material (niobium in the case of our niobiumtitanium wire) is introduced as a normal-conducting phase in the supecionducting material. The flux lines are localized to these regions. Artificial flux pinning allows some measure of control over the final microstructure of the superconducting material-an intrinsic characteristic that cannot be altered

\footnotetext{
* Generally speaking, NMR refers to the nonimaging scientific tool and to the underlying physical principle, whereas MRI refers to the imaging technigue used for applications such as medical diagnosis.

** Nole that the ll-T record mentioned carlier was for an accelerator-type magnet. The magnets for high-energy accelerators must exert their field over a considerable length in order to bend or focus the extremely "rigid" particle beam, and the field must be extremely homogeneoun. Their design is also constrained by many mechanical and comomic hactors. NMR magnets, although challenging in their own way, are much different physically, so the apparent tremenderus leap from $10 \mathrm{~T}$ in accelerator magneds $1023.5 \mathrm{~T}$ in this project is misleading.
}

Nonaccelerator Applications

\author{
APC Materials \\ Development
}


by the way the superconducting material is formed into wires. (The significant intrinsic factors are filament microstructure and composition. Extrinsic factors, such as the cross-sectional area, integrity, and uniformity of the superconductor, are also important.) Figure 5-7 illustrates this prir.ciple.

The ultimate application of this research is to enable the fabrication of multifilamentary superconducting wire that has higher critical-current density $\left(J_{c}\right)$ and is more economical to produce. We are examining niobium distribution and pinning strength as key intrinsic factors that may offer opportunities for further understanding and progress. We are also working with several industrial companies on ways of producing APC superconductor by the strictly mechanical means of cold-working, rather than the timeconsuming and expensive heat-treatment technique that is used today.

In 1991 and 1992 we studied two such approaches, selecting one proposed by Supercon, Inc., that improves the high-field performance of the APC superconductor and also promises to reduce the cost of the material. Microstructural examination of the wire (Figure 5-8) showed that it had a uniform structure despite a local area ratio (copper matrix to superconductor filaments) of about 0.6 . Such a low ratio would have caused "sausaging" with conventional manufacturing techniques. It is desirable to make this ratio as low as possible to increase the $J_{1}$ of the wire. (Superconducting magnets retain the desired electrical properties only up to a certain critical

\section{Conventional Flux Pinning Artificial Flux Pinning (APC)}

Figure 5-7. In conventional flux pinning (left) the fluxoids are localized at the randomly distributed points where precipitates were formed by heat treatment. (These sites are elongated, as shown here, when the material is drawn into wire.) In artificial flux pinning, normalconducting metal sheets are interleaved with the superconductor in the manufacturing process and drawn down to nanometer scale. This provides better matching between the pinning sites and the flux lines. It also obviates the formation of pinning centers as a reason for heat treatment.

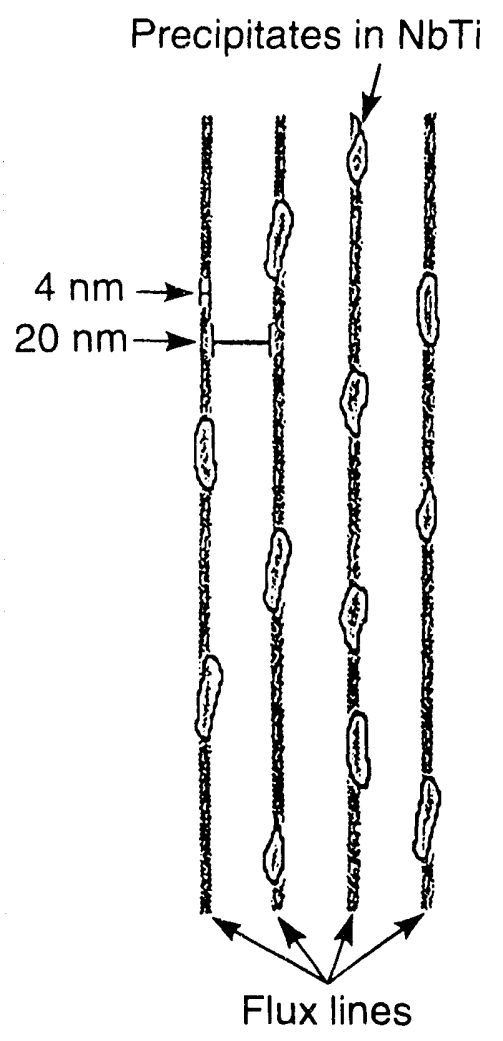

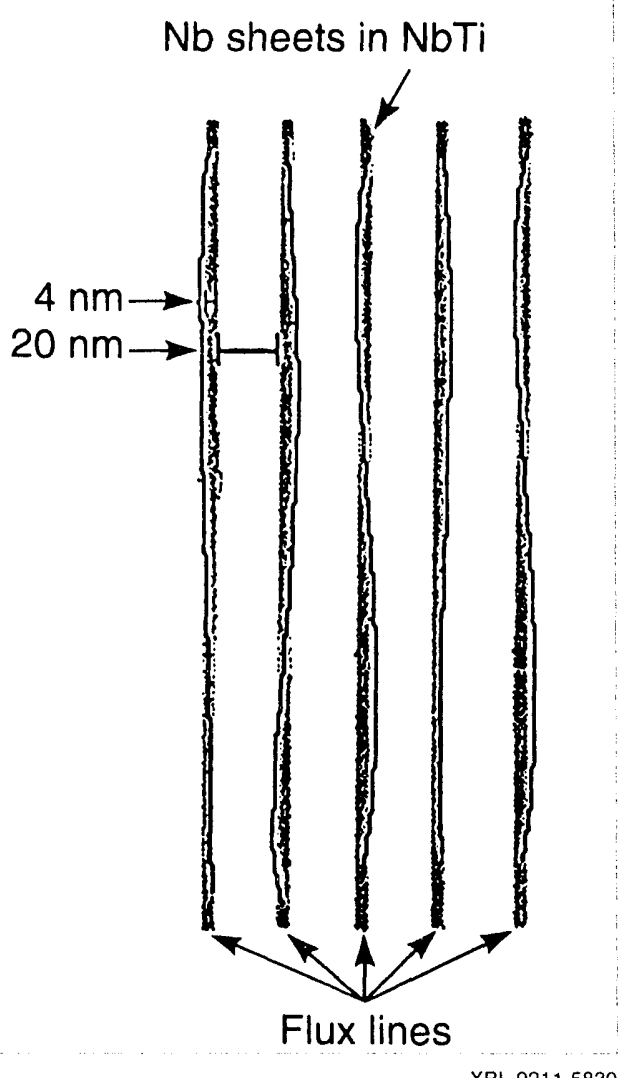



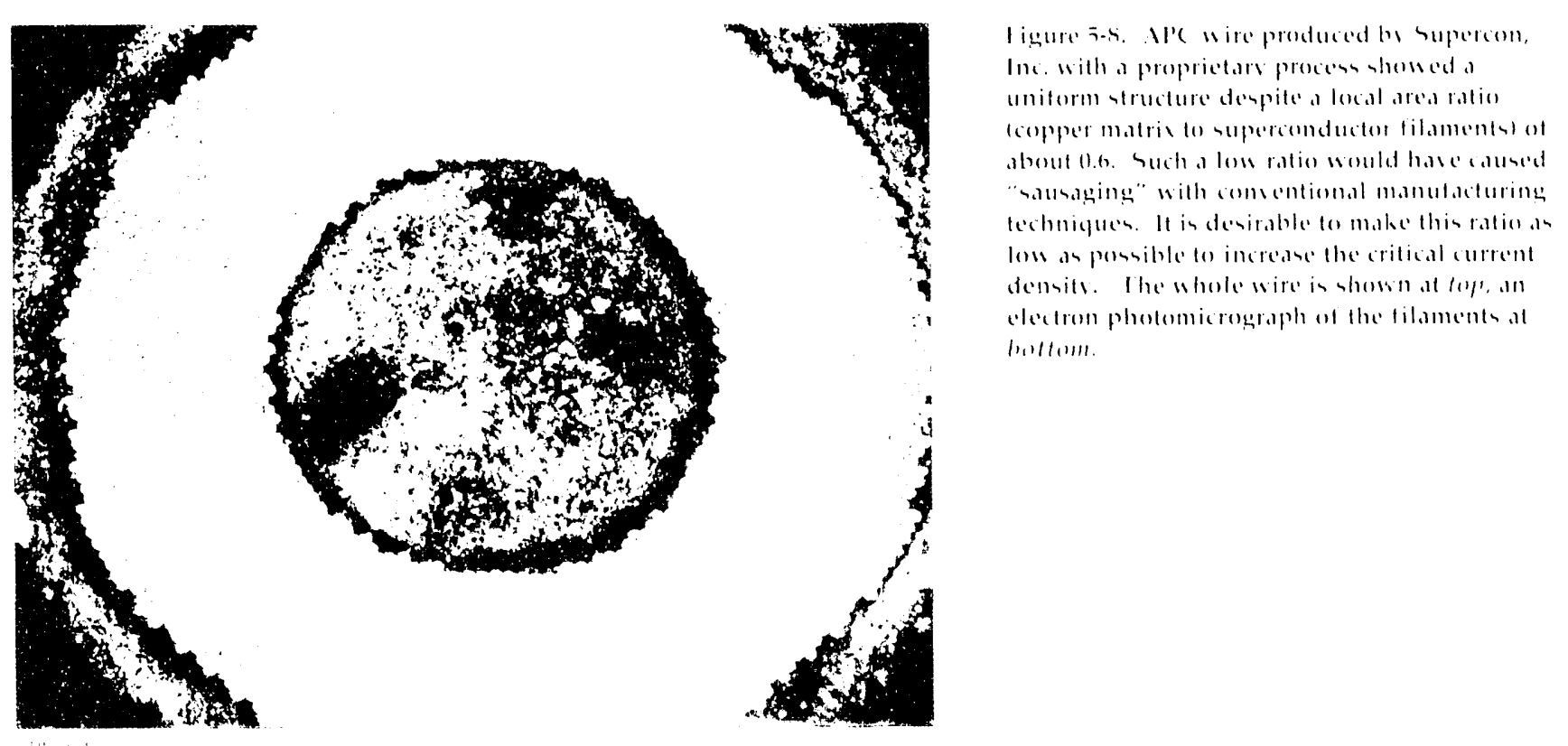

(arrent: ahene that hend, thes

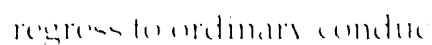

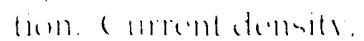

temperitture. and molsmeth

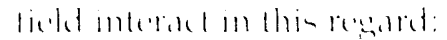

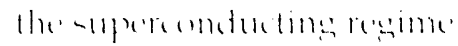

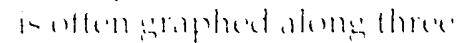

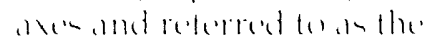

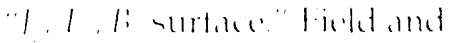
arment atem-1ts a , m he limited the fmotamental preperticent the -

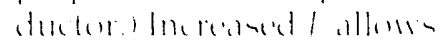

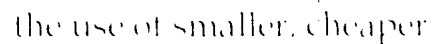
ablear, altermateds, the. aherement at hisher todets

herelemold homon in

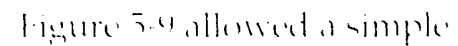

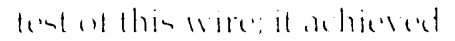

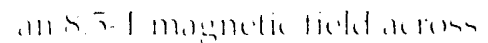

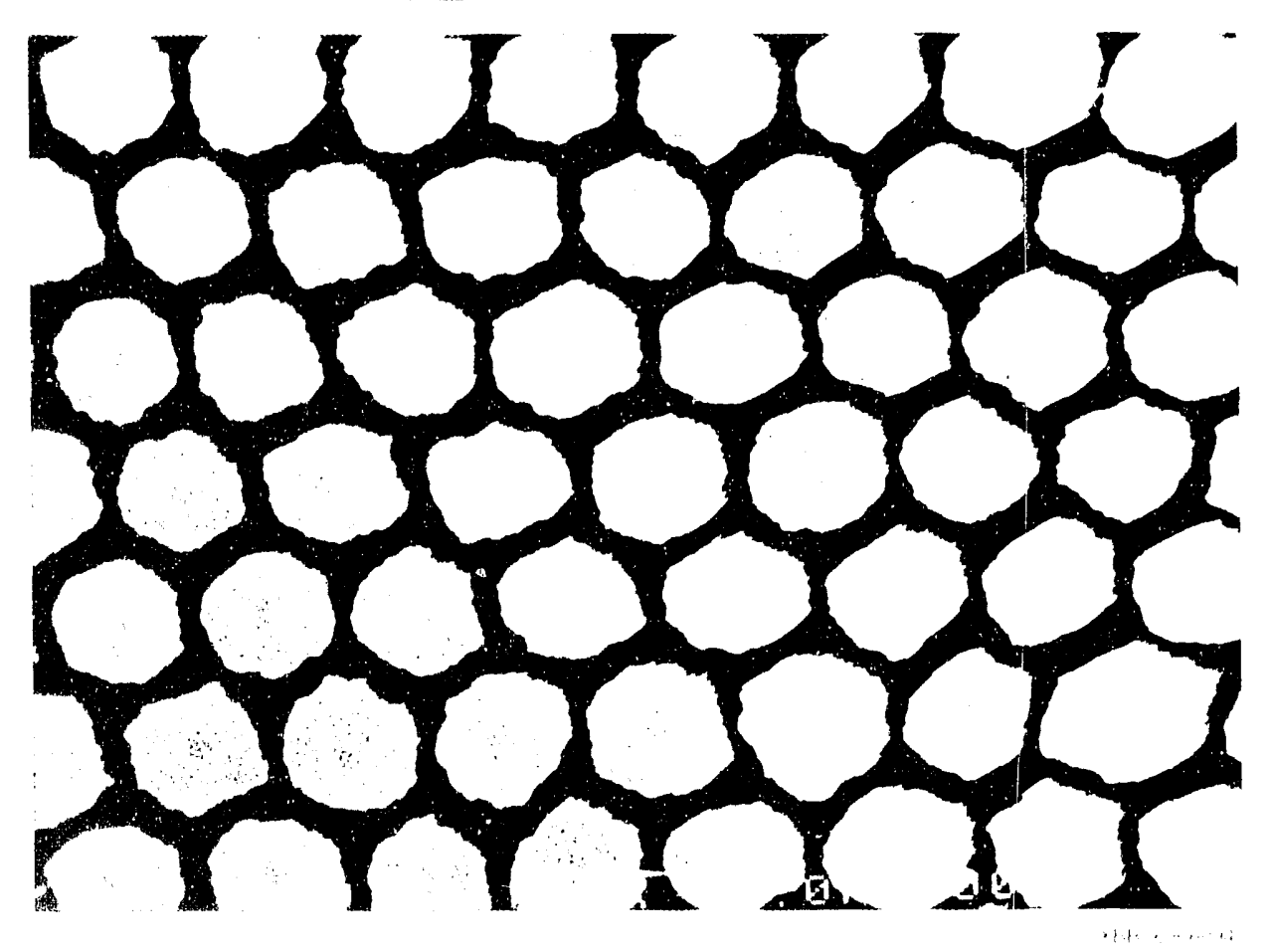
it 15 mml horediameler at

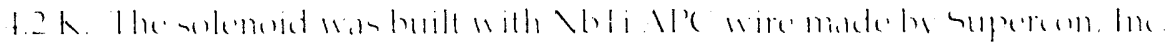

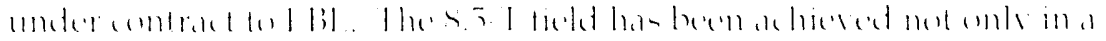

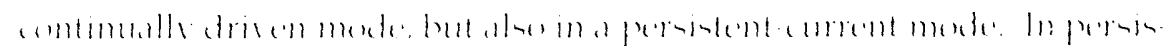

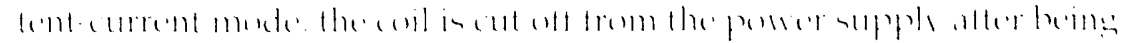

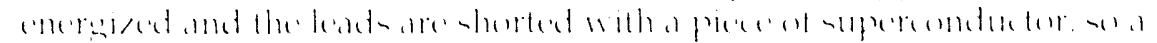

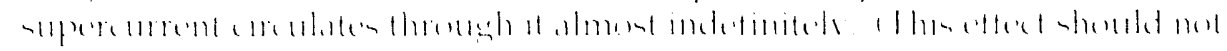

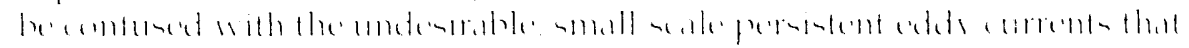

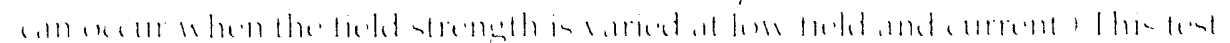

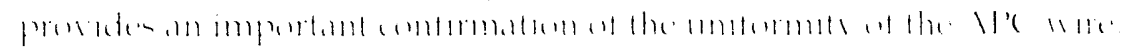


Figure 5-9. This 8-T solenoid was built as a simple test of the new wire from Supercon, Inc., shown in Figure 5-8. Tests with persistent current (continuing circulation of a current after the power supply was disconnected) provided an important confirmation of the uniformity of the APC wire. As a result of this successful demonstration, we have ordered enough of the APC wire to fabricate cable and build a 1-mlong dipole, D19B, that will give us further information on the performance, reliability, and cost-effectiveness of this type of material.

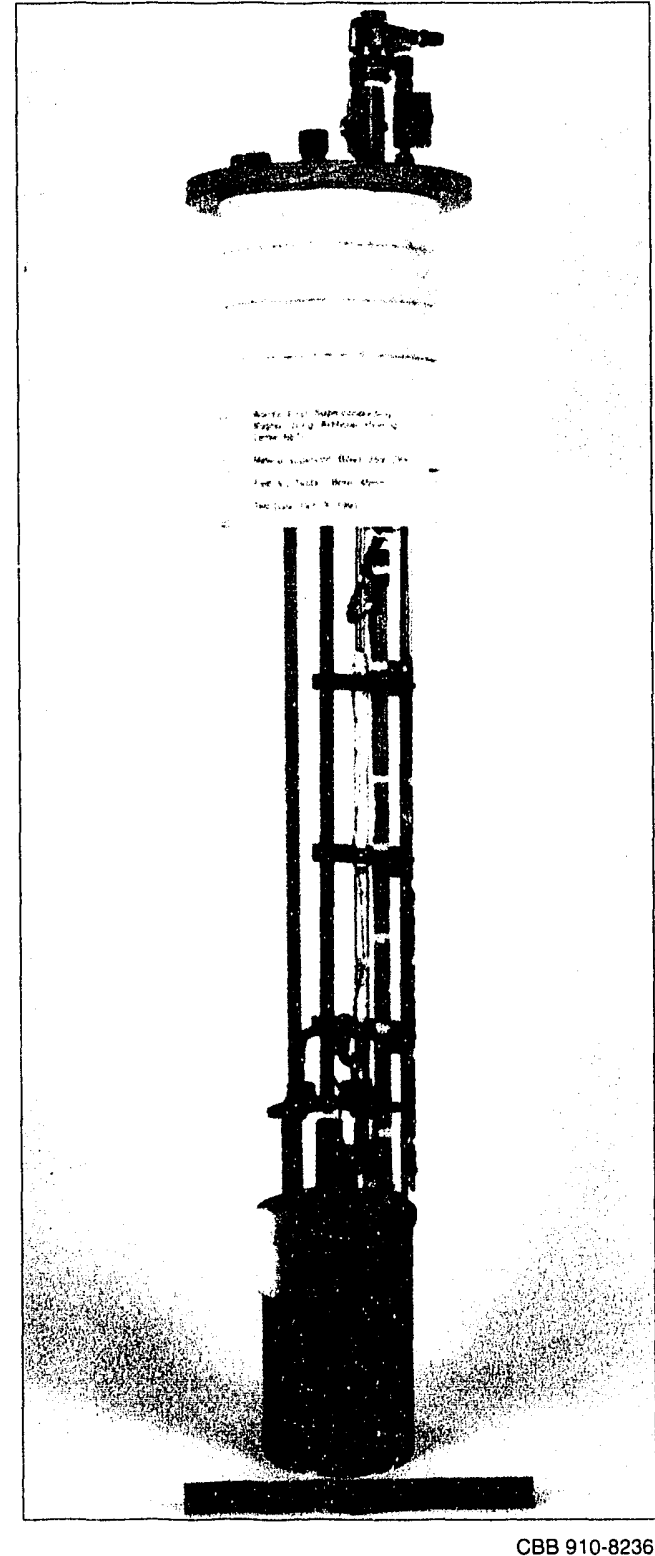

As a result of this successful demonstration, we ordered enough of the wire to fabricate cable and build D19B. Testing this 1-m-long dipole magnet will give us further information on the performance, reliability, and costeffectiveness of APC material, which is needed to realize the full potential of $\mathrm{NbTi}$ and may prove useful in $\mathrm{Nb}_{3} \mathrm{Sn}$ and other brittle superconductors as well.

High- $T_{c}$

Superconductor at Low Temperature
Although nearly all the emphasis in the program has been placed on traditional Bardeen-Cooper-Shockley superconductivity, we also have a promising investigation into so-called high- $T_{\mathrm{c}}$ superconductors. These materials, which we actually use at liquid-helium temperatures in order to achieve high magnetic fields, might someday be useful for accelerator and other magnets. 
However, many challenges will have to be overcome in order to turn them into useful engineering materials for devices such as accelerator magnets that must withstand great stress or must be built up into complicated shapes.

In an effort currently funded with Laboratory Directed Research and Development monies (and interesting to at least one potential industrial partner), we are examining phase equilibria and microstructural texture in thick films of the bismuth oxide ( $\mathrm{BiSrCaCuO})$ family of compounds. In particular, we are studying ways of creating and preserving an aligned microstructure in the $\mathrm{Bi}_{2} \mathrm{Sr}_{2} \mathrm{Ca}_{1} \mathrm{Cu}_{2} \mathrm{O}_{2}$ or " 2212 " phase throughout the phase transformations that occur during processing, both within the bismuth-oxide compound and between it and its silver substrate. Earlier work indicated a $/$. of greater than $1000 \mathrm{~A} / \mathrm{mm}^{2}$ for 2212 bismuth-oxide material. This figure seems to be on the same order as the $J_{\text {c }}$ of NbTi conductor (albeit at a low magnetic field), though the sample was too small for incorporation in a coil. Whether formed into tapes for windings or deposited onto surfaces as a coating (e.g., for superconducting rf cavities in accelerators), high- $T_{\text {. }}$ compounds represent a promising area of development.

A longtime goal of our superconductor R\&D program has been to develop improved techniques and tooling for the fabrication of Rutherford-style cable. The R\&D cabling machine we developed to meet the needs of the SSC, and which is now available in a commercial version, continues to serve our R\&D needs. In 1991 we upgraded it with a new spool and Turk's-head so that it could fabricate cables with as many as 48 strands of superconducting wire. With this new equipment, we have been able to fabricate cables that are $30 \%$ wider and contain $25 \%$ more strands than the cable we developed in the late 1980s for the SSC dipole magnets. In addition to furthering cable manufacturing, this upgrade has provided our magnet designers with additional flexibility in their choice of superconducting materials and cable designs.

The easy in-house availability of this machine, along with the expert assistance of its operators, has paved the way for innovative materials experiments. For example, we will use it to make the 48 -strand $\mathrm{Nb}_{3} \mathrm{Sn}$ cable for D20; the able can be made in the usual fashion because the wire is ductile. Later, after magnet winding, it is heat-reacted in place; this coincidentally makes it brittle.

In the attempt to better integrate two quite different fields that depend on one another, we are exploring an analytical approach to magnetic design. The goal is to use the same notation that is common in beam dynamics, expressing the three-dimensional fields produced by electromagnetic lenses in terms of a single function $A_{4}(Z)$ and its derivatives. Thus far, by applying the Biot-Savart law and using both a differential algebra package developed in part at LBL and a numerical method, we have achieved success in threedimensional modeling of magnetic fields. A longer-term goal is to extend this technique to guide accurate conductor placement and to use simulation codes to study the resulting particle motion.

\section{Cable and Cabling- Machine Development}

Analytical Magnet
Design 


\section{Publications and Presentations}

P. Barale, "Quadrupole centering correction," abstract accepted for the International Industrial Symposium on the Super Collider (San Francisco, CA, 1993); LBL-33012a (1992).

S. Caspi, "An approach to 3D magnetic field calculations using numerical and differential algebra methods," LBL-32624 (1992).

S. Caspi, "Non-linear beam dynamics," Spring Meeting of the American Physical Society (Washington, DC, 1992); LBL-31719a (1992).

J. Cortella, "Mechanical performance of fullscale prototype quadrupole magnets for the SSC," Applied Superconductivity Conference (Chicago, IL, 1992); LBL_-32788 (1992).

D. Dell'Orco, "A $50 \mathrm{~mm}$ bore superconducting dipole with a unique iron yoke structure," Applied Superconductivity Conference (Chicago, IL, 1992); LBL-32071 (1992).

D. Dell'Orco, "Design of the $\mathrm{Nb}_{3} \mathrm{Sn} 13 \mathrm{~T}$ dipole D20," Applied Superconductivity Conference (Chicago, IL, 1992); L_BL-32(172a (1992).

D. Dietderich, "Noble metals and $\mathrm{Bi}_{2} \mathrm{Sr}_{2} \mathrm{Ca}_{1} \mathrm{Cu}_{2} \mathrm{O}_{4}$ " Applied Superconductivity Conference (Chicago, IL. 1992); LBL-321(14a (1992).

D. Dietderich, "Nuclention and growth of $\mathrm{Bi}_{2} \mathrm{Sr}_{2} \mathrm{Ca}_{1} \mathrm{Cu}_{2} \mathrm{O}_{4}$ in textured thick films," Materials Research Society (Boston, MA, 1992); LBL-32635a (1992).

M.A. Green, "UCLA Phi Factory detector, the integration of superconducting compensation solenoids and the final focus interaction region quadrupoles," International Cryogenic Engineering Conference (Kiev, Ukraine, 1992); L.BL-326.35 (1992).

A. Lietzke, "Quadrupole magnet for the SSC," Applied Superconductivity Conference (Chicago, IL, 1992); LBL-32790) (1992).
A. Lietzke, "SSC quadrupole magnet performance at LBL," abstract accepted for the International Industrial Symposium on the Super Collider (San Francisco, CA, 1993); LBBL-33002a (1992).

J. Royet, "Recent developments in cabling technology used to manufacture superconducting accelerator magnets," LBL-32069a (1992).

R. Scanlan, "Characterization \& coil test results for a multifilamentary $\mathrm{NbTi}$ conductor utilizing artificial pinning center technology," Applied Superconductivity Conference (Chicago, IL, 1992); LBL-32074 (1992).

C. Taylor, "Construction and test results on $40 \mathrm{~mm}$ bore $5 \mathrm{~m}$ long quadrupole magnets for the SSC," LBL-32074a (1992).

J. van Oort, "A fiber-optic strain-gauge with absolute readout using the strain-dependent frequency-shift of the transmission spectrum of a fused biconical tapered $2 \times 2$ coupler," LBL-32623a (1992).

J. van Oort, "A superconducting pressure test-station for material property tests at 4.2 K," LBL-32620a (1992).

J. van Oort, "An absolute fiber-optic strain gauge using white-light interferometry of an extrinsic fiber-optic Fabry-Perot cavity," LBL32622a (1992).

J.M. van Oort, "The application of extrinsic Fabry-Perot fiber-optic strain sensors in accelerator dipole magnets," LBL-32184a (1992).

J. van Oort, "The design of a $1.3 \mathrm{~T}, \mathrm{Nb}_{3} \mathrm{Sn}, 1 \mathrm{~m}$ model dipole using a window-frame coil layout," LBL-32621a (1992).

J. van Oort, "The reduction of the critical current in $\mathrm{Nb}_{3} \mathrm{Sn}$ cables under transverse Ioads," Applied Superconductivity Conference (Chicago, IL, 1992); LBL-32070 (1992). 


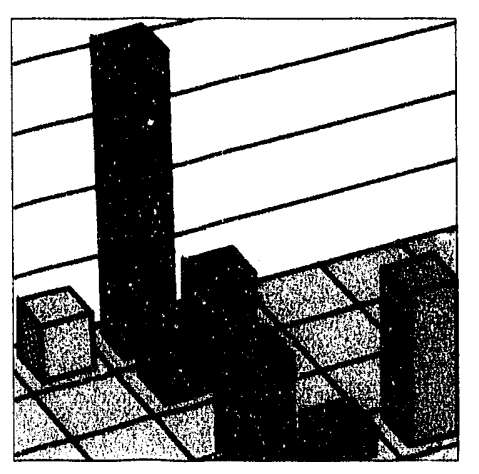

6.

\title{
BEVALAC OPERATIONS
}

\begin{abstract}
After nearly fOUR DeCades of SERVICE and several important discoveries, it appears likely that operations at the Bevalac will stand down completely in early 1993. Previously we had expected that the principal funding source, the DOE Division of Nuclear Physics, would withdraw its funding in 1994, whereupon a new sponsor would take advantage of the accelerator's capabilities. The Bevalac advisory committees (Tables 6-1 and 6-2) have guided us in the painful task of deciding which already-approved programs should be pruned to make the best use of the remaining year of operations. Meanwhile, the Bevalac continues its traditional mission of serving nuclear science, radiation biology, and clinical radiation treatment.

Research teams and the operations staff are working hard to make the best use of the available beamtime while attempting to identify other venues for the vast amount of work that has yet to be done. The nuclear science
\end{abstract}

B. Feinberg (operotions program he'ad: planning and development group leader) C.F. Krebs (restarch comatimation sroupl later) H.K. Syversrud toperutions graltip lender)

R. Stevenson (soffety and admintstrative siritit's

siction liater)

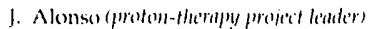

S. Abbott**

R.V. Aita

D. Andersen ${ }^{* *}$

L. Archambulul**

P. Arhart**

J.1. Ayers**

B.). Bailey**

M.O. Balaget**

R. Barr

D. Beck**

(i. Behrsing**

M. Bennett**

D.A. Bentsen

R. Berminzoni*

1. Bercosity**

A. Biocena*

M. Blasbalg $\left(s^{2} m t^{\prime} s\right)^{* *}$

K. Bolin ${ }^{4 *}$

M. Bordua**

\begin{tabular}{|c|c|}
\hline J.P. Brannigan & R. Engstrand* \\
\hline M.J. Bricker, Jr.* & 1. Etchells* \\
\hline W. Brown** & W.L. Everette \\
\hline W.L. Bruemmer* & W. Faust** \\
\hline I.A. Brusse*** & M. Felix* \\
\hline J.P. Burch** & K. Fetters** \\
\hline W.E. Byrne & 1. Finsch** \\
\hline R.L. Callaway ${ }^{3}$ & I. Flores** \\
\hline J. Calvert** & R. Force**a \\
\hline M. Casey ${ }^{* *}$ & K. Fowler** \\
\hline I. Castey** & R. Frias**s \\
\hline C. Colata & DS. Ciarrield** \\
\hline R. Celestre** & B. Caving \\
\hline 11.J. Chamberst* & F.E. Cevisler** \\
\hline W. Chism* & T.L. Cimpel \\
\hline W.T. Chu' & J.D. Ciregorr** \\
\hline R. Conte's & J.R. Ciuggemos \\
\hline D.N. Cowles & B. $H_{a} \|^{*}$ \\
\hline 11. Crawford (gutst) th & 1. Harmon** \\
\hline A.W. Cutt ${ }^{*}$ & W. Harndent* \\
\hline S.L. Daly & 1. Henderson ${ }^{* *}$ \\
\hline R.L. Dote** & E. $H(m) s(m)^{* *}$ \\
\hline J.R. Dougherty** & D. Howard** \\
\hline R. Dwinell** & P.M. Howeslo \\
\hline J.A. Elkins** & D. Iluctson** \\
\hline H.M. Ellison & M. Hfui** \\
\hline
\end{tabular}

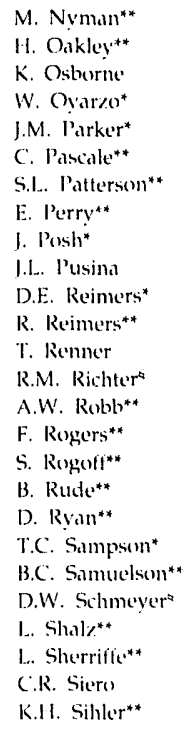

R.P. Singh*t

R. Sorrensent*

W. Spedrse*

J.W. Staples

I.I. Stout **

R. Stradtuer $r^{* *}$

W. Strelot*

S.A. Stricklin**

D.L. Syversrud**

M.M. Tekawa

R.K. Thatcher

J.R. Thomas**

P. Torres"

A. Tranis**

P. Varghatt+

J.C. Walling*

M. Williams*

M.D. Wolfe

C. Woud*

K. Woulfe*t

E. Zajuce

J. Zukuwski**

Administrative Support

b. Finch

R. Perry

I. Mibonald

* Plant Engineering and Construction and Maintenance Departments

** Enginecring Division

+ Research Medicine and Radialion Biology Division

"University of Californin Space Sciences Laboratory"

it Envirmment, Health, and Salely Division

setired 
community has inaugurated a new Equation of State detector, which is taking advantage of a development program that effectively more than doubles the available number of research hours.

The Bevalac lives on in a variety of ways, though, including its legacy of spinoff projects and unexpected benefits. One of the latest is a proton-therapy project being considered by the University of California at Davis, which has drawn heavily on the accelerator and biomedical expertise associated with the Bevalac. The accelerator, which would be built at their cancer center in Sacramento, would be designed in a cooperative effort involving oversight from LBL scientists and engineers and strong industrial participation.

Table 6-1. Program Advisory Committees (PACs) for 1992.

\section{Bevalac Nuclear Science PAC}

S. Datz, Oak Ridge National Laboratory

B. Feinberg, LBL, operations manager

C. Gelbke, Michigan State University

F. Goldhaber, State University of New York at Stony Brook

M. Gyulassy, LBL

W. Henning, GSI, Darmstadt, Germany

G. Krebs, LBL research coordinator

F. Lothrop, LBL scheduling coordinator

P. McMahan, LBL executive secretary

F. Plasil, Oak Ridge National Laboratory

R. Scharenberg, Notre Dame University, users' representative

L. Schroeder, LBL, scientific director

V. Viola, Indiana University, chair

\section{Bevalac Biomedical PAC}

S.J. Adelstein, Harvard Medical School, chair

Eleanor A. Blakely, LBL, deputy executive secretary

J.D. Chapman, Cross Cancer Institute

William T. Chu, LBL, executive secretary

Edward R. Epp, Massachusetts General Hospital

B. Feinberg, Bevalac operations manager (ex officio)

Robert J. Michael Fry, Oak Ridge National Laboratory

David J. Grdina, Argonne National Laboratory

G.F. Krebs, Bevalac research coordinator (ex officio)

Robert E. Krisch, University of Pennsylvania

Amy Kronenberg, LBL

Lester J. Peters, University of Texas System Cancer Center

Bernhard Ludewigt, LBL, biomedical experiment liaison

Table 6-2. Nuclear Science Users' Association Executive Committees for 1992.

P. Brady, University of California at Davis

J. Carroll, University of California at Los Angeles

D. Keane, Kent State University

A. Mignerey, University of Maryland

M. McMahan, LBL, executive secretary

D. Olson, LBL

H.G. Ritter, LBL, scientific director (present)

R. Scharenberg, Notre Dame University, chair

L. Schroeder, LBL, scientific director (past)

These panels of experts recommend beamtime allocations. The LBL scientific director of each program may allocate about $10 \%$ of the beamtime on a discretionary basis. 


\section{Accelerator \\ Technology and Operations Summary}

1992 Performance Statistics
A steady program of technology upgrades, combined with long experience at efficient scheduling and with continual fine tuning of operating procedures, has helped the Beralac staff ozercome a long-term trend of decreasing budgets coupled with inflationary erosion. With closure immine'nt, maintenance dountime zons reduced in 1992, bringing the Beralac up to 3122 hours of experinental beamtime, up from 3000 hours in 1991. (The sustained trend in the late 1980s had been 4000-plus hours of experimental beamtime per year.)

Many factors affect beam delivery, including scheduled and unscheduled shutdowns for maintenance, usage for machine studies and tuning, and seasonal fluctuations in the cost and availability of electricity (traditionally, the facility shuts down during part of the summer). Despite these variations, there have been steady improvements. During 1992, the impending shutdown reduced the need for maintenance, further increasing net operating hours. Additionally, we continued a scheduling evolution called "block running" that began several years ago, in which nuclear physics is given long, uninterrupted blocks of beamtime, punctuated by long periods reserved for radiotherapy alone. This has allowed the most efficient use of the limited time available.

The physical sciencesmostly nuclear physicscontinued to use about twothirds of the experimental beamtime at the Bevalac. The life sciences, comprising radiotherapy research and basic radiobiology and radiation biophysics studies, accounted for the rest. Table 6-3 summarizes the year's operating statistics and compares them to figures for past years.
Life Sciences

SuperHILAC*

Total

Number of nuclear-science

experiments receiving beam

Number of participating scientists

Institutions represented

Universities

National laboratories

Other

Totial

Use of Beamtime (\%)

In-house staff

Universities

DOE national laboratories

Other institutions

\begin{tabular}{|c|c|c|}
\hline $\begin{array}{r}1991 \\
\text { actual }\end{array}$ & $\begin{array}{r}1992 \\
\text { actual }\end{array}$ & $\begin{array}{r}1993 \\
\text { projected }\end{array}$ \\
\hline 2975 & 3122 & 1440 \\
\hline 225 & 269 & 24 \\
\hline$\underline{706}$ & 773 & 192 \\
\hline 3906 & 4164 & 1656 \\
\hline 459 & 444 & 360 \\
\hline 4395 & 4176 & 6744 \\
\hline 4854 & 4620 & 7104 \\
\hline 2034 & 2045 & 1392 \\
\hline 941 & 1077 & 48 \\
\hline 400 & $\underline{0}$ & $\underline{0}$ \\
\hline 3375 & 3122 & 1440 \\
\hline 8 & 12 & 3 \\
\hline 64 & 64 & 24 \\
\hline 15 & 18 & 9 \\
\hline 3 & 5 & 0 \\
\hline$\underline{2}$ & $\underline{3}$ & $\underline{0}$ \\
\hline 20 & 26 & 9 \\
\hline 50 & 50 & 25 \\
\hline 35 & 35 & 75 \\
\hline 5 & 5 & 0 \\
\hline 10 & 10 & 0 \\
\hline
\end{tabular}

* SuperHILAC operition was for a single experiment previously approved but unable to rum until FY91. 
The Bevalac (Figure 6-1) has an ongoing program of technology upgrades designed to increase efficiency, improve user service, and ensure safety. A multiyear project to modernize the Bevalac's control systems and extend modern computer systems throughout the facility saw additional progress in 1992. The computer control system has been extended to the local injector's rf system and drift-tube-magnet power supplies and to the beam transport and diagnostic devices in all beamlines. Conversion from the obsolete ModComp computer control system to the new system, which is based on networked Sun workstations, also progressed.

Our 1992 accelerator-improvement projects were directed toward raising the maximum "flat-top" time of the main guide-field magnet's power supply, and therefore the maximum beam spill time, from 1 second to as

\section{Facility Development Projects}

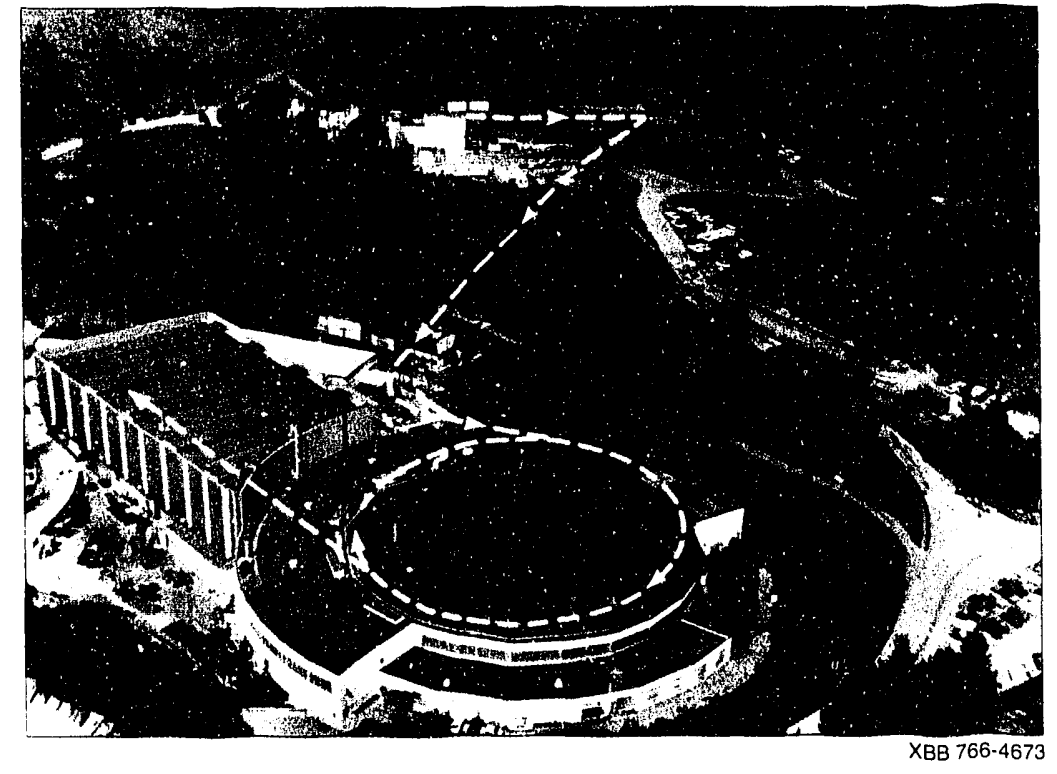

Figure 6-1. The Bevatron, a synchrotron, accelerates beams from either its local injector or the SuperHILAC heavy-ion linear accelerator. The dotted lines in the picture show the beam path from the SuperHILAC through the Bevatron. The SuperHILAC, which has three complementary ion sources, is used when higher energies and heavier ions are needed, as in most of the nuclear-physics programs. The beams are delivered to a variety of users in the External Particle Beam hall. The faint dashed lines in the drawing correspond to the circular and rectangular buildings shown in the photograph. The various experimental areas are separated by a "maze" of shielding blocks.

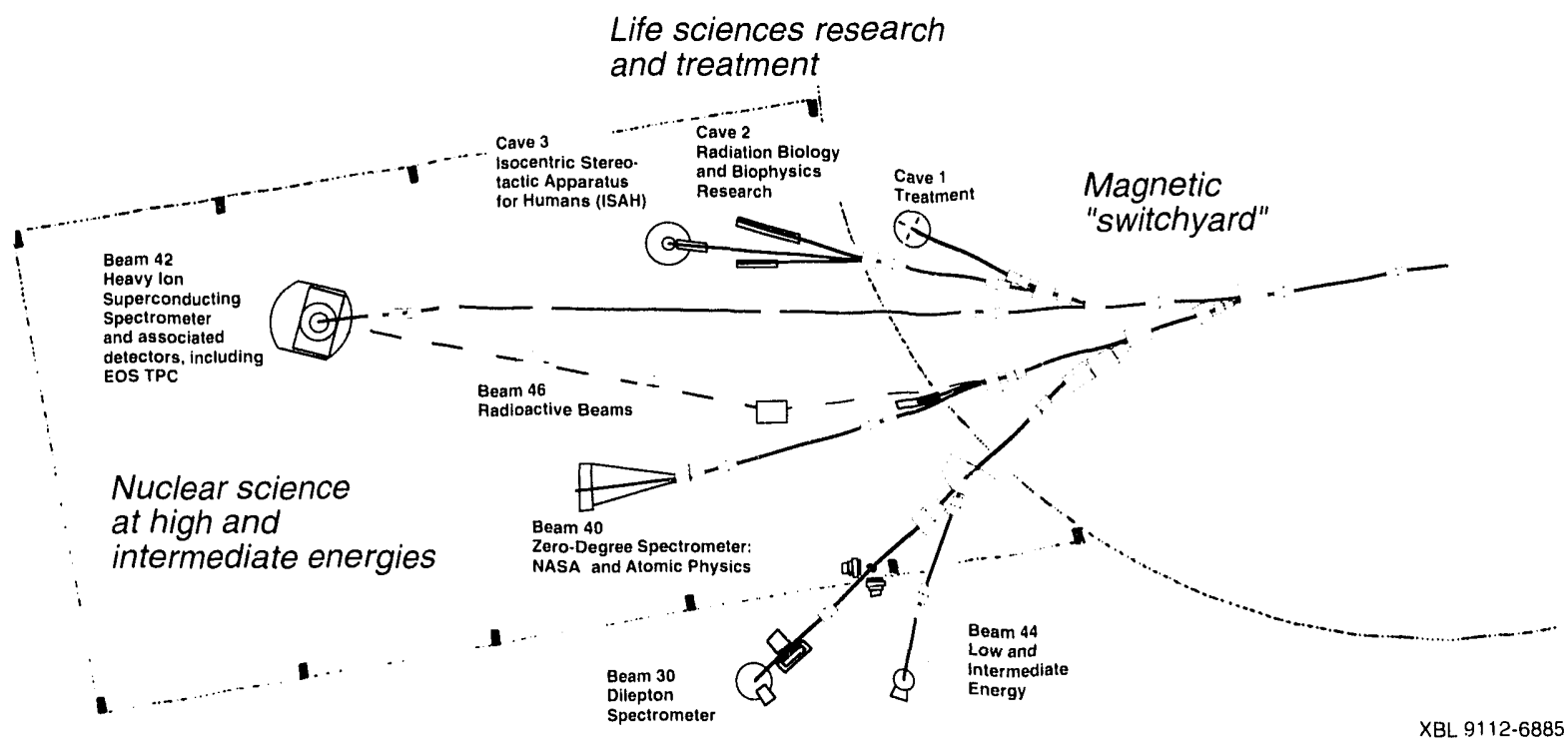


much as 9 or 10 seconds. This project required R\&D for new measures to stabilize the magnetic field and to substantially reduce power-supply ripple. This has allowed the Bevalac to greatly increase the overall duty cycle-by up to a factor of three-thus providing service that better matches today's emphasis on high-statistics investigations rather than survey experiments. The primary nuclear physics experiments have made great use of this new feature.

\section{Proton Medical Accelerator}

\author{
The Davis Initiative
}

The use of accelerated ion beams to reach tumors precisely in three dimensions is deeply rooted in the history of $L B L$. Over four and a half decades, radiotherapy with heavy, charged particles has become widely accepted for the treatment of an eve;increasing variety of tumors. Meanwhile, LBL accelerator scientists and their lifesciences colleagues have been thinking from time to time about special-purpose accelerators deticated to medical use. In recent years these troo lines of inquiry have come together in a proton-therapy initiatize planned for the University of California at Davis Cancer Center, located in Sacramento. This facility will be the second dedicated, hospital-based, clinical charged-particle treatment facility in the world, following the lead of the Loma Linda University Medical Center. The present schetule calls for its commissioning for clinical use in 1997.

The Davis facility - formally, the University of California at Davis Proton Therapy Center-will be a $250-\mathrm{MeV}$ proton accelerator optimized for medical treatment. Specifications and design decisions for the Davis facility are being developed with the aid of grants from the National Cancer Institute.

In 1992 the growing enthusiasm for the project culminated in the signing of a memorandum of understanding between LBL Director Charles Shank and UC-Davis Chancellor Theodore Hullar. LBL continued to develop specifications for clinical requirements (together with Davis and Massachusetts General Hospital) and accelerator performance (in cooperation with Davis and the University of California at San Francisco). These specifications will be used in seeking formal approval from the UC Board of Regents and then, probably in early 1993, for issuing a Request for Proposal to the vendors that are interested in bidding on the actual engineering design. Among the key areas of inquiry are techniques for creating large radiation fields suitable for therapy, along with isocentric gantry delivery of the beam. LBL is among the leaders in this field (see the Biomedical Research section of this chapter); one of the challenges before us is to transfer our knowledge and technology to Davis and to private industry before the closure of the Bevalac, and, if possible, to identify funding sources that will keep this experienced and uniquely qualified team intact.

Meanwhile, in the second year of NCI funding, Davis worked on site-specific architectural, managerial, regulatory, and operational aspects of a design for the Sacramento facility. The Proton Therapy Facility, to be located in a new structure adjoining the recently completed Cancer Center, is currently envisioned as a $250-\mathrm{MeV}$ proton synchrotron delivering beam to three treatment rooms, two of which are equipped with rotary gantries capable of bringing the treatment beam into a supine patient from any angle. Throughout these design efforts, we are taking advantage of lessons learned from the construction and operation of the Loma Linda facility in the hopes of achieving true second-generation performance at Davis. 
In a parallel activity, approval has been chtained for moving the Bevalac eve-treatment program to the (rocker Nuclear Laboratory on the Davis campus. The haburatory operates a direct descendant of one of E.O. Lan'rence's original machines, the Crocker (n)-Inch Cyclotron, which was moxed to Davis from Lawrence's laboratory on the Berkelev campus. Its 68MeV proton beams are ideal for the treatment of shallow tumors, such as ocular melanomas. Crocker Nuclear Laboratory persomel and LC Davis management are enthusiastically supporting the establishment of this program at their hacility, and work is currently underway to adapt hardware and technigues developed at the Beralac. Patient treatment is expected to start in mid-1493.

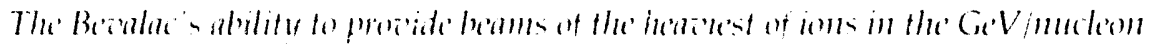

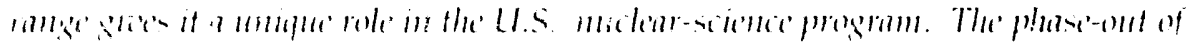

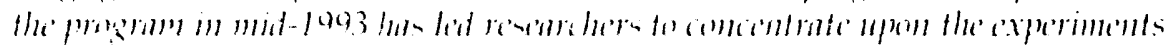

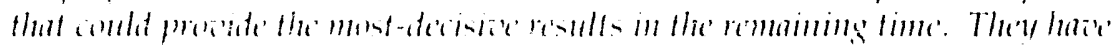

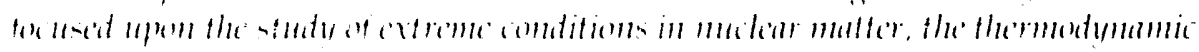

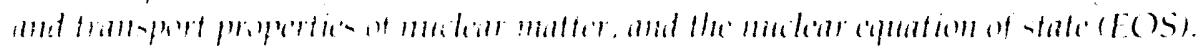

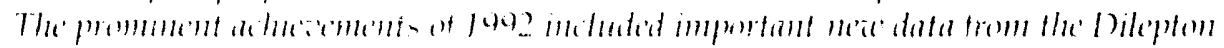

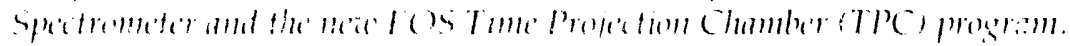

The rumme theme of the Besalace nuclear seience program has been the production ad a amination of extreme conditioms in nuclear matter. Early Bewalace periments, using the Streamer C hamber and Plastic Ball detectors, establinhed that central : Ilinions between muled could oreate high tempera-

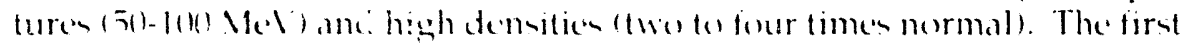

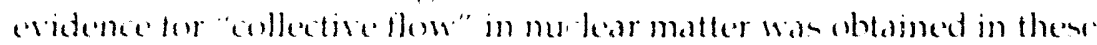
eperments. the ntwdies reveal the thermodenamic and transport proper-

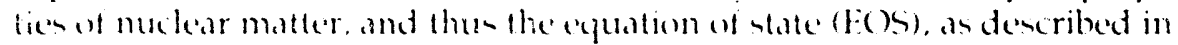
the idebar. Howewer. the effective comprensibility of nuckear matter remained uncertain to within a tactor of 2, so clearly a consensus on the form of the loc had not been reathed.

Eyperience has - town that, te reach a unique description of the collision

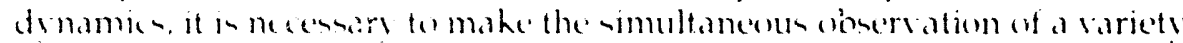
ot nuckear precenes and to apply a combistent theoretical tormalism to fitting the data. What in icepured in at detector capatse of analezing most of the tharged particle produed in a cen'ral collinion between the hedsiest nuclei. At the same time, this detecter meut be able to collect a large statistical

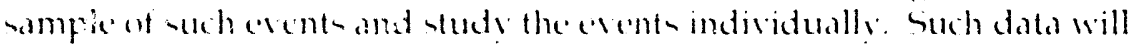
emable the extriction of seurce temperature trom the energy spectro, source premeren trom the collective momentum thew, source entropies trom the

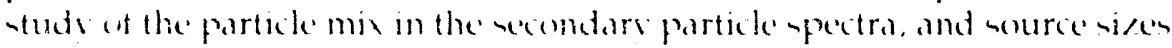
trem l tanburs Brown and Twin meanurementeon poire ot identical par-

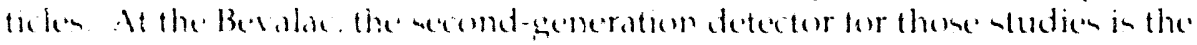
ros I!c itme projection chamber).

\section{Nuclear Science}

\author{
EOS Studies and the \\ Time Projection \\ Chamber
}

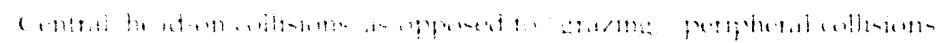


The EOS TPC (Figure 6-2) fits inside the Heavy Ion Superconducting spectrometer (HISS) magnet. The detector is designed for full-solid-angle coverage, hence the term " $4 \pi$." It is able to identify and analyze the momenta of the mid-rapidity charged particles (mostly protons, deuterium and tritium nuclei, ${ }^{3} \mathrm{He}$ and the nuclei, and pi mesons) that are produced when heavy nuclei such as gold collide at Bevalac energies. The experiment is completed by using a variety of existing HISS detectors like the multiple sampling ionization chamber (MUSIC), a time-of-flight scintillator wall, and a neutron detector.

In 1992 we started an extensive program that measures excitation functions for a variety of symmetric and asymmetric target-projectile combinations. A summary of the number of events accumulated for all the combimations is shown in Figure 6-3. Work will continue until the termination of the nuclear science program at the Bevalac.

In addition to EOS studies, the

Pamping and Commenessing the Nuclear Hoid

Some of the effects of nuclear collisions are rather exotic, as are the means of observation. However, many of the effects are analogous, in eonsiderable detail, to phenomena one sees in everyday, macroscopic matter. (An example of such behavior is collective ilow, which was first observed in the Plastic Ball and Streamer (hamber detectors at the Bevalac.) Nuclear matter can be thought of as a solid, liquid, gas, or plasma, depending on temperature and pressure, as shown in Figure 6-4. The equation of state (EOS) mathematically describes the balance among these phases and the borderlines of the phose transitions. Knowledge of the FOS is of great fundamental importance to muclear scientists. It is also useful to astrophysicists, because certain hypotheses about mechanisms within supernovae and neutron stars are based on assumptions related to the EOS. The hypotheses are especially sensitive to the value of nuclear incompressibility.

Probing the EOS under conditions far removed from the equilibrium state of nuclear matter requires considerable disturbance of the entire volume of interacting matter. This calls for head-oli impacts from beams of heary ions. The greater the mass of the target nucleus and the projectile, the greater the disturbance and the greater the number of participating nucleons. Therefore, an ongoing highlight of Bevalac research is exploration of the EOS at high temperatures (fypically 5()-100) MeV of thermal energy) and high densities (fwotofour times normal).

The Bevalac energy range is especially appropriate hecause regions of the Eos far irom equilibrium can be reached, yet the phenomena are still strengly influened by the nuclear mean field. In other werds, the nucleus still behaves as a unit in the collision, whereas at much-higher energien, such a those of the Relativistic lleavy-lon Collider being built at Brookhasen National Laboratory, the effects are expected to resemble those of elementary particles striking each other.
EOS TI' is used to examine nuclear multifragmentation. This phenomenon can be studied through a complete experiment in charge space (identifying the total initial charge independent of impact parameter) using the technicue of reverse kinematics. This work involves heavy beams such as krypton, hanthanum, and gold upon lighter targets such as bervllium and carbon. 

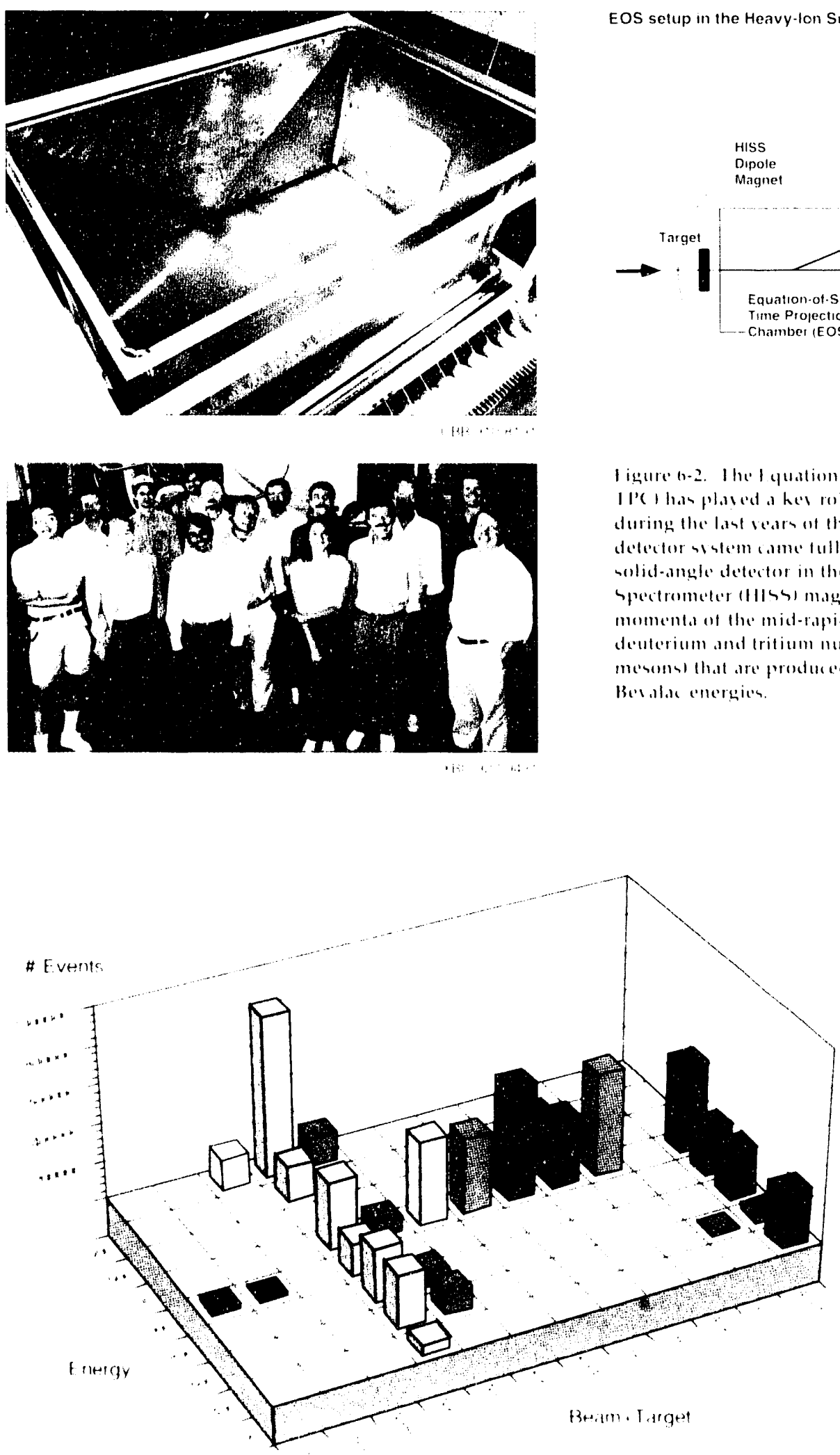

EOS setup in the Heavy-Ion Superconducting Spectrometer (HISS) system

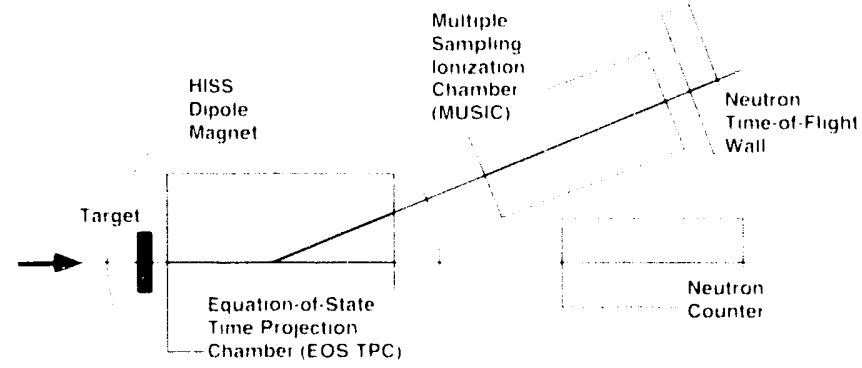

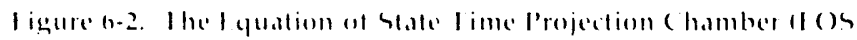
1 l' 1 has phased a her role in optimising siontitis progress during the lant veare ent the Beralde nuclear-acience progham. The

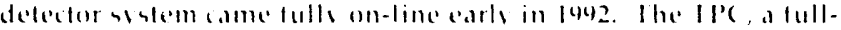
solid-angle detector in the lleas lon hupercombluting

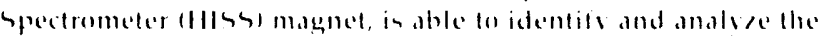
momenta of the mid-tapidits charged partiden lmontle protoms. deuterium and tritium muled. 'He' and 'He nuclei, and pi mesoms that are produced when hean muclei inch collide at Bersilac energien.

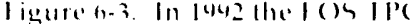
collabordtion started an enternate program that meanduresencitation functions tor a d ariels of

stometris and asmometrie targed. propedete combindtions lhis shaph summoristen the number of

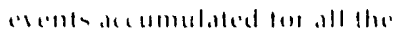

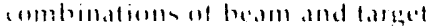
clements 
Figure 6-4. A schematic phase diagram for nuclear matter shows some of the states and transformations predicted by various theories. The normal state of nuclear matter can be characterized as liquid. It may undergo phase transitions to a hadron gas at relatively low densities or, at higher densities, to some condensed phase such as a pion condensate or a superdense nucleus containing thousands of nucleons. Under extreme conditions, the hadrons themselves are expected to break down into "deconfined" quarks and gluons; such conditions are thought to have existed in the first few fractions of a second after the Big Bang, and are thought to exist today in neutron stars and supernovae. Such extreme conditions can be re-created at a laboratory scale through central collisions between nuclei at extreme relativistic energies.

Dilepton Spectrometry

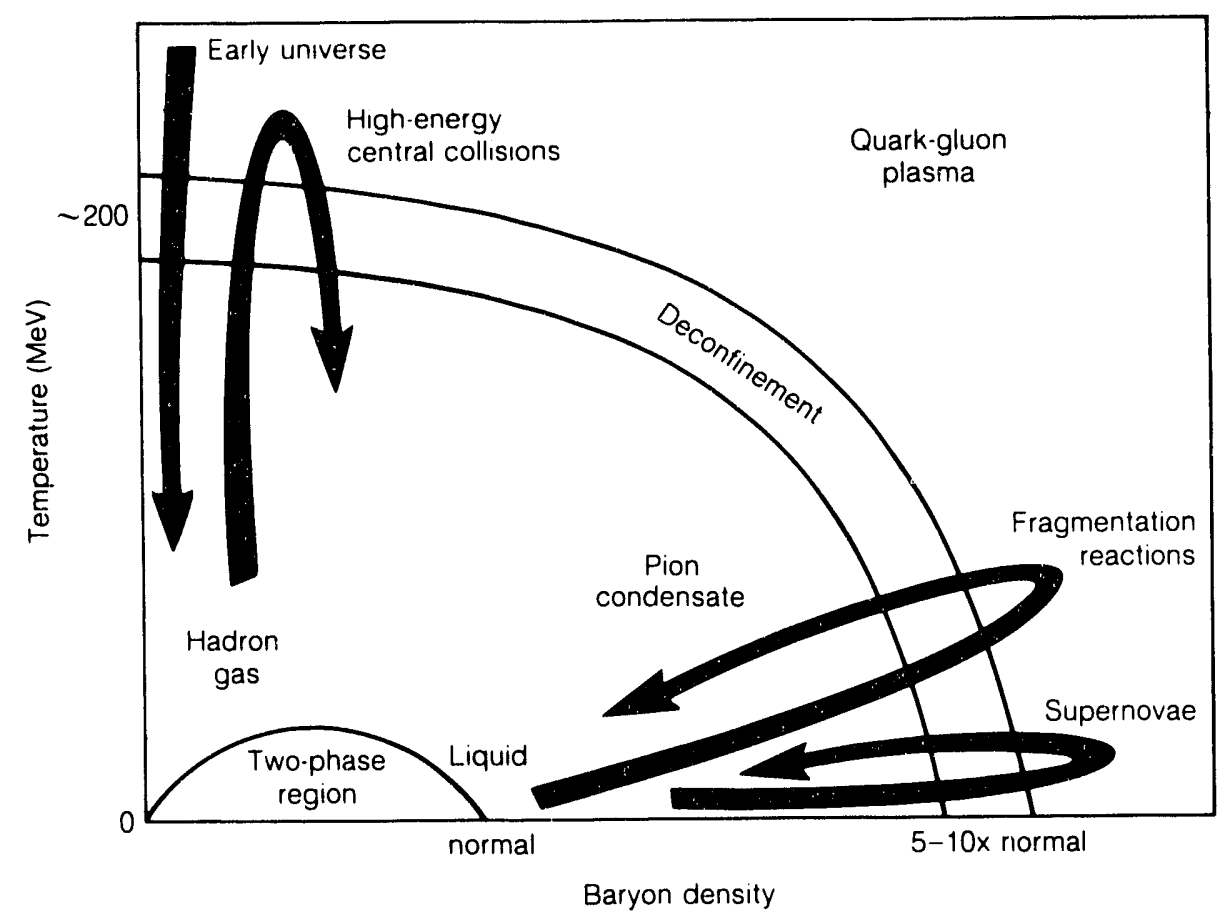

XBL $881-8818$

Another important detector is designed to provide an especially clear view of certain reactions. The Dilepton Spectrometer (DLS), shown in Figure 6-5, is a unique detector that was installed at the Bevalac in 1986. It offers special insights into reaction dynamics by watching for a rare event: emission of an electron and positron correlated in their paths and their time and place of origin.

Dilepton emission is thought to provide an especially clear view of nucleus-nucleus, proton-nucleus, and proton-proton collisions because leptons interact with other forms of matter through the weak nuclear force and the electromagnetic force, not the strong nuclear force. Thus there is only a small probability of scattering or reabsorption on their way out of the reaction area, and reliable data can be obtained on deep and early phenomena of the collision. In particular, dilepton spectrometry might provide insights into one of the key theoretical unknowns of nuclear collisions: the behavior of pions and other mesons. (It is also thought that dilepton production might signify the formation of a cuark-gluon plasma in the higherenergy heavy-ion colliders of the future.)

In 1987 the DLS collaboration established that dilepton emission does indeed occur at Bevalac energies. Subseguent work further defined the role of dilepten spectrometry in studying the EOS and other behavioral aspects of hot, compressed nuclear matter. Many models have been proposed to explain the early DIS data.

To verify preliminary interpretations of these DLS data, it was essential to measure the dilepton spectrum for the elementary nucleon-nucleon case. A licpuid hydrogen/deuterium target was added to the DLS. In these collisions, which involve elementary nucleons rather than nuclei with a collective 


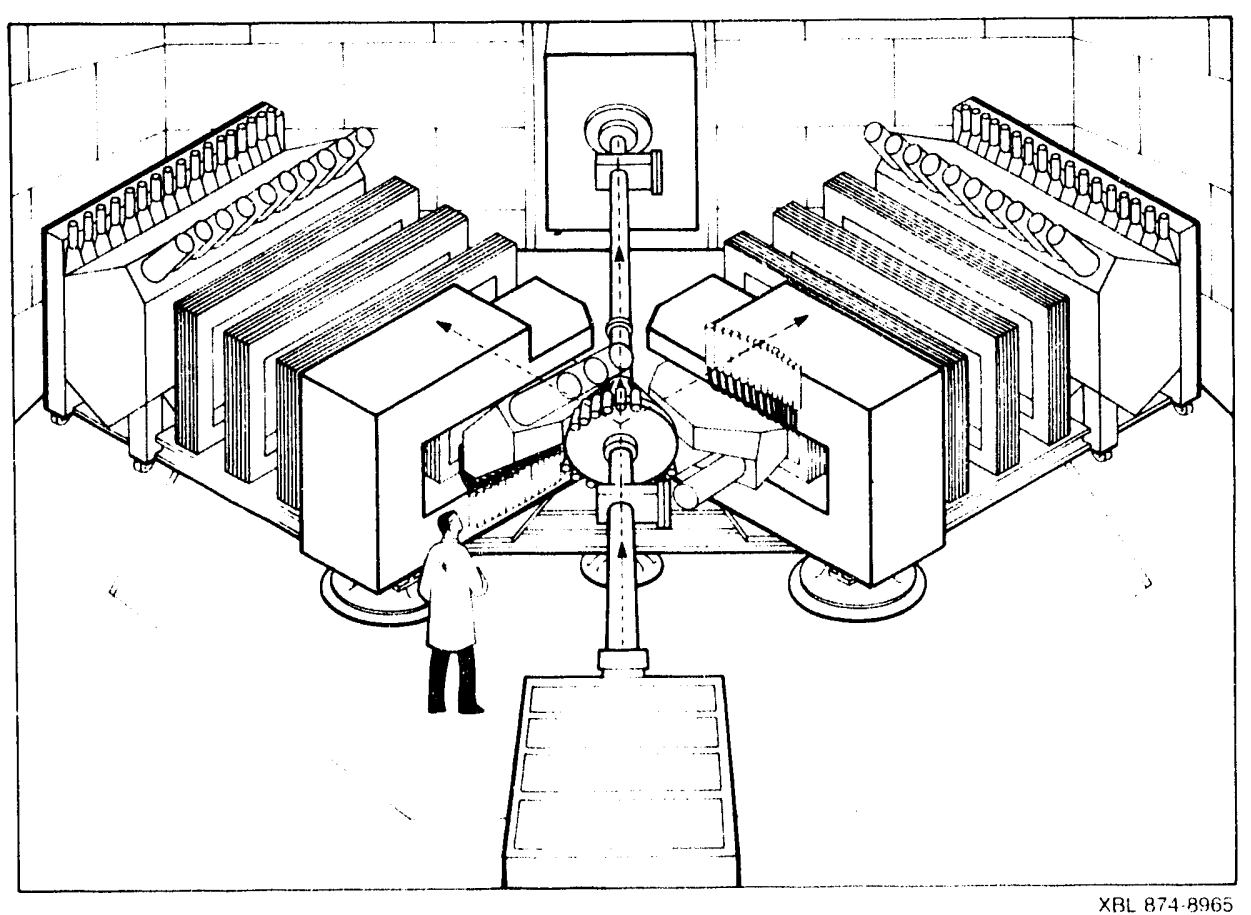

"mean field," it could be shown that proton-neutron Bremsstrahlung does not account for a large part of the dilepton pairs produced, as predicted by most of the model calculations. The main contribution comes from resonance decay such as the Dality decay of the 17 meson.

In 1492 the DIS collaboration finished the work on the high-statistice p-p and p-D study. About $200(0)$ true pairs were accumulated. Then the group continued to study dilepton production in nucleus-nucleus collisions. This will reveal whether the structure observed in the data truly reflects a property of the nuclear medium.

Figure 6-6 shows the cumulative number of true dilepton pairs measured with the DLS over the years. Note the way that operational and accelerator improvements, such as block running and improvements in the time structure of the beam spill, have led to a dramatic increase in scientific productivity in recent vears.

Recent theoretical speculation suggests that collective flow should set in at energies as low as 50-100) MeV/n. Earlier data from a Streamer (hamber experiment carried out by researchers from Michigan State University apparently show a disappearance of flow at energies below $70 \mathrm{MeV} / \mathrm{n}$. In 1991, a group from Kent State University began an extensive set of measurements of the triple differential cross section for neutrons produced in the collisions of the heaviest systems, attempting to ascertain the energy at which flow disappears.

Inclusive charged-particle experiments, carriced out using the two single-arm spectrometers at the end of Beam 31), are in the final stages of analy'sis uning new data sets. These data, take'n with high precision, should be sensitive te effects of the nuclear medium and should also serve as a testing ground for theoretical models of contral nuckeus-nucleus collisions for hedry sy'stems.
Figure 6-5. An artist's rendering shows the major elements of the Dilepton Spectrometer (DLS), which in its completed and installed form is difficult to photograph. Electron-positron pairs are detected in the two arms, each of which has a three-cell Cerenkov gas counter, a 16element scintillation hodoscope, drift chambers and a magnetic dipole, a 20-cell Cerenkov gas counter, and a large 16-element scintillation hodoscope. In 1989, an additional multiplicity detector was added: a 96-element hodoscope configured as a cone surrounding the target chamber. This hodoscope detects charged particles and gives information about the centrality of the event.

Neutrons and Light Charged Particles 
Figure 6-6. Dramatic increases in the scientific productivity of the Bevalac have been achieved in recent years through such measures as block running and improvements to the time structure of the beam spill. This graph of the cumulative number of true dilepton pairs measured with the DLS over the years illustrates this trend.
Grazing Collisions and Secondary Radioactive Beams

Nuclear Astrophysics, Atomic Physics, and NASA Instrument Calibration

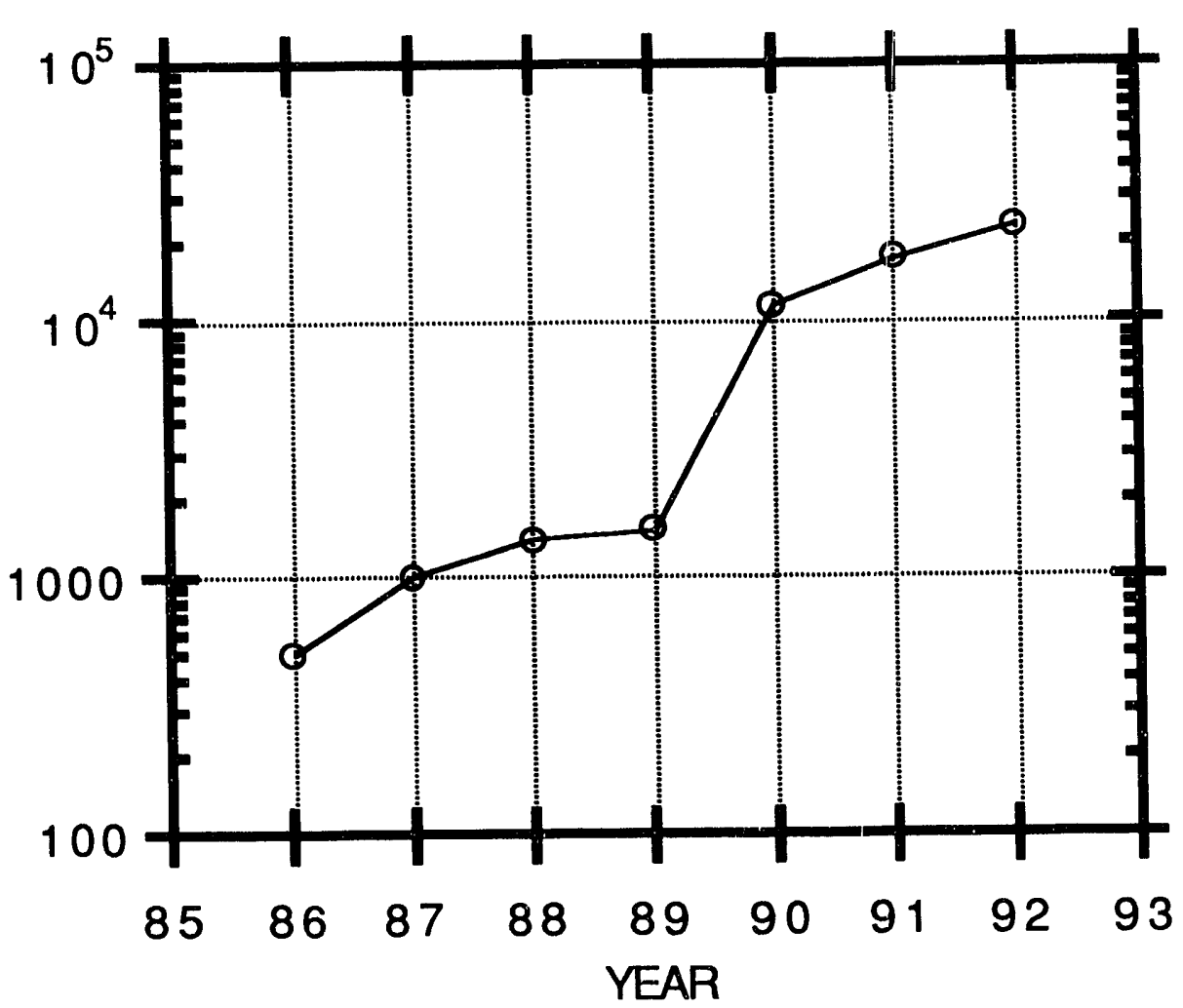

XBL.931-4703

Although most Bevalac research focuses upon the extreme temperatures and pressures caused by central collisions, other extreme phenomena can be studied in "grazing" collisions. For example, nuclear fragments with extreme numbers of protons or neutrons-out to the "driplines" at the edges of the chart of the nuclides-can be created. Considerable progress continues to be made in using the projectile fragmentation processes to produce and study beams of radioactive nuclei. Initial studies with "Li showed clear evidence for a two-component structure, which has been interpreted as a sign of a diffuse neutron skin (halo). This has been followed by studying the A-dependence of the electromagnetic-dissociation (EMD) process for "Li, an isotope for which a large EMD component was observed.

Many of the Bevalac's capabilities are relevant to astrophysics. HISS is used to study nuclear reaction mechanisms and to measure heavy-ion inclusive fragmentation cross sections. This holds particular interest for those who study cosmic-ray propagation in the interstellar medium. The group performing this research is measuring fragmentation cross sections for projectiles as heavy as Fe colliding with targets of liquid hydrogen and helium (the elements that are the principal components of the interstellar medium).

A variety of work is done at the Bevalac in disciplines other than nuclear science. Bevalac beams are used for instrument calibration by researchers from the National Aeronauties and Space Administration (NASA) and by 
other cosmic-ray scientists from around the world. They use the beams to calibrate detectors that will be used on balloon, rocket, and satellite flights and to recalibrate them after retrieval.

Atomic physicists use the Bevalac's ability to provide "hydrogen-like" and "helium-like" uranium ions (that is, ions stripped down to one or two electrons). Because uranium has 92 protons, these highly stripped ions represent an extreme condition of the atom. On their small scale, they have the strongest electric fields found in nature, enabling scientists to address phenomena in quantum electrodynamics, including the Lamb shift.

Production of electron-positron pairs in collisions of fully stripped uranium nuclei on gold targets, with the electron captured by the outgoing nucleus, has consequences for RHIC, the Relativistic Heavy Ion Collider being built at Brookhaven National Laboratory, and has been explored in a recent experiment at the Bevalac. And a gas-cell target was developed at the Bevalac to measure charge-changing cross sections for ions that are of interest to the designers of new accelerators at CERN and Brookhaven; it also examines the role of electrons in relativistic ionizing collisions. This gas cell was then used to study resonant transfer and excitation for $\mathrm{U}^{91+}$ projectiles in hydrogen. These experiments provide a test of relativistic dielectronicrecombination theory.

In addition to nuclear science, a dienese bionedical program has thrived at the Beralac. This section describes selected 1992 highlights in the three primary areas of Beralac biomedical work:

- Clinical programs in henoy-charged-particle radiation Horapy and radiosurgery.

- Radiation biology and biophysics research.

- Derelopment of e'quipme'nt and teclmique's.

Other life sciences research at the Beralac, accounting for about $20 \%$ of bionedical beamtime', include's basic studies of the effects of ratiation on both normal and abnormal cells and tissues. In zizo and in vitro experiments examinin' such subjects as damage and repair of DNA, cell and tissue kinetics, and radiation-induced tumor genesis. This zerok is relewant to many fields, including extended manned space exploration beyond the Earth's protectioe magnetosphere. Biomedical researchers and clinicians who zerork at the Berulac, like their collengues in muclear science, are working furiously to continue their programs after the early-1993 shutidouln, searching for me'ue ze'nue's and making arrangements for the tracking of patients.

A program of teclmology deselopment, aimed at safer and more effectize delizery of the prescribed radiation doses, has gone hand in hand aith the'se research and treatment efforts. In 1992, Research and De'olopment Magazine recognized the Raster Scammer berm-dclizery syste'n with an RED 10() areard, given to the year's 10) most-significant tedunology dea'elopments. The Raster Scanmer, commissioned for clinical use' in 1991, is a significant ste'p toneards dynamic thre'-dime'nsional conformal atelizery.

Clinical research medicine at the Bevalac focuses primarily on Bragg-peak radiation treatment (Figure 6-7). Two general types of treatments are carried out: radiosurgery of intracranial arteriovenous malformations (AVMs) and radiotherapy of tumors.

\section{Biomedical \\ Research}




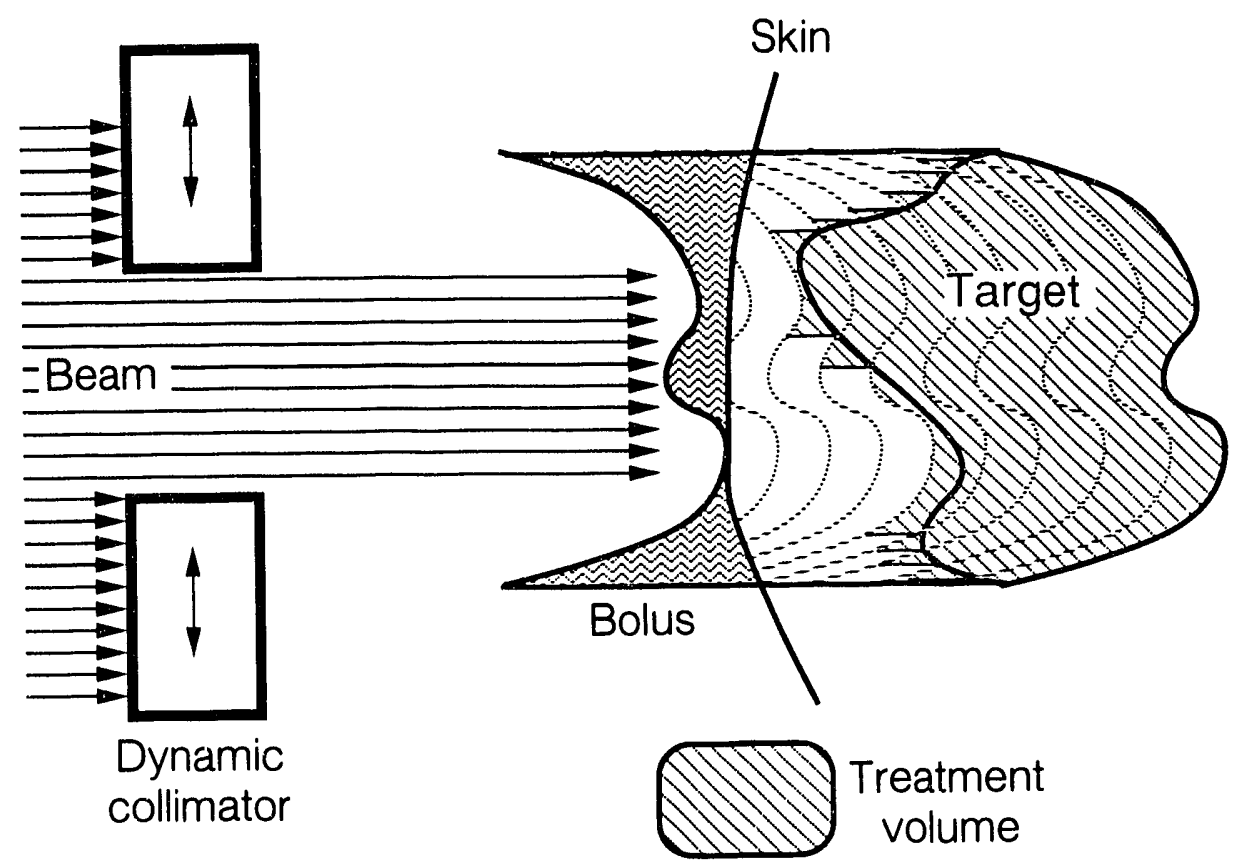

XBL 896-6557

Figure 6-7. The Bragg peak gives charged particles an advantage over electromagnetic radiation (such as $x$-rays) for radiosurgery. Electromagnetic radiation grows weaker exponentially as it is absorbed, so delivering an effective dose to a deep tumor means considerable damage to healthy tissues in front of and behind the tumor. Particles, by contrast, lose most of their energy in a relatively narrow part of their range; the location of this "Bragg peak" can be accurately predicted

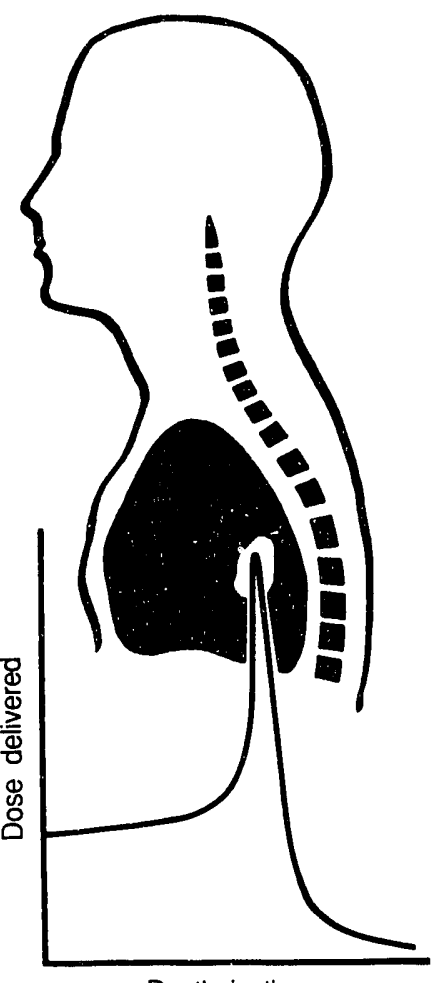

Depth in tissue

XBL 737.969 and precisely controlled. In the radiosurgical instrument arrangement diagrammed here, an energy-absorbing wax or Lucite bolus matches the depth of the Bragg peak to the thickness of the tumor, while collimators control the cross section of the beam. Not shown is an upstream "binary filter," or absorber, which draws the beam back in sequential layers. Changing these variables can fit the treatinent area to the tumor across three dimensions.

Since 1980, more than 470 patients with symptomatic, inoperable intracranial AVMs and other vascular disorders have been treated at LBL with stereotactic Bragg peak radiosurgery. This program of helium-beam treatment, in which we collaborate with medical centers in the area, was initiated at the nowdecommissioned 184-Inch Synchrocyclotron and then moved to the Bevalac. A long-term dose-searching clinical-trial protocol has followed more than 250) patients for more than 2 years. Initially, radiation doses ranged from 45 to 35 gray-equivalent $(\mathrm{GyE})^{*}$ now, doses of 25,20, 15 and, under special circumstances, $10 \mathrm{GyE}$ can be used, depending on a number of factors.

In these AVM treatments, radiosurgery functions as one of several tools, and the goal is to prepare the patient for subsequent procedures. The radiosurgery reduces the vascularization of an inoperable AVM so that it can be treated by other means, such as embolization or microneurosurgical resection. When the entire arterial phase of the AVM core is included in the treatment field, the rates for complete obliteration 3 years after treatment are

The gray is a unit of energy depesition by ionizing radiation: 1 joule per kilogram of mass. It in equivalent 10 100 rads. The gray-eguivalent accounts for differences in relative biolegical effectiveness caused by the "quality factor" of different kind of radiation (ions, neutrons, rays, and so forth); the linear energy transfer (LET) of the radiation being used; and the vulnerability of the tissue being irradialed. 


\section{BEVALAC OPERATIONS}

impressive: $90-95 \%$ for volumes of $4-14 \mathrm{~cm}^{3}$ and $60-70 \%$ for volumes greater than $14 \mathrm{~cm}^{3}$. The total obliteration rate for all volumes up to $70 \mathrm{~cm}^{3}$ is approximately $80-85 \%$. For complete radiation-induced obliteration, dose is primarily related to volume, secondarily to location. The implementation of raster scanning in Cave III opens up the possibility of treating complex intracranial lesions that have previously been impossible to treat effectively, even with beams of charged particles.

A related helium-ion bioeffects research program continues to investigate the reaction to heavy-charged-particle radiation injury. Advances in dosimetry and beam-delivery technologies provide a better understanding of dose localization and dose distribution in the Bragg ionization peak at selected sites. Emphasis is placed on the cellular basis of central-nervoussystem damage and repair. Research addresses the biophysical events of DNA damage and repair; oligodendrocyte, neuronal, and endothelial-cell kinetics and homeostasis; perturbations of regional blood flow dynamics and regulatory control; and cell population kinetics under heavy-ion irradiation.

The success of the charged-particle radiosurgery program at LBL has led to the development of a Cooperative Research and Development Agreement (CRADA) between LBL, Loma Linda University Medical Center, and Stanford University Medical Center. Under this CRADA, the LBL-Stanford developments in technology and technique are being transferred to the hospital-based proton facility at Loma Linda. Researchers at the three institutions will conduct cooperative research, concentrating on optimizing the radiosurgical treatment of patients whose AVMs are large or complex in shape. Selected basic-science programs at LBL will also be transferred to Loma Linda. The scheduled closure of the Bevatron has made it necessary to expedite the technology-transfer process, since more than 10) AVM patients are currently awaiting treatment with charged particles and no feasible alternative to the Bevatron is currently available.

Radiotherapy has continued to be another highly successful aspect of the Bevalac biomedical programs. By the end of 1992, more than 1350 patients had been treated in LBL clinical trials, which began in 1975. After the shutdow'n, the approximately 600 living patients will be followed for the next 5 years to further evaluate results and late effects. This tracking program, supported by the Department of Energy and the National Cancer Institute, will help assure optimal use of the expertise developed at LBL over the past 15 years.

The clinical trials with helium ions in skull base and juxtaspinal tumors continue to show excellent results as compared to historical control data, as does a randomized trial of uveal melanoma in the eve in which helium was compared to 27 plaçue therapy. The rate of complications is acceptably low and remains comparable with that of standard radiotherapy.

The initial local control results with neon ion irradiation in the Phase I-II studies have been promising, with rates ranging from 50-9)\% for salivary gland, locally advanced prostate, advanced poranasal sinus and nasopharynx tumors, as well as for locally residual sarcoma of bone or soft tissue. We continued to search for additional evidence regarding the clinical effectiveness of high-linear-energy-transfer (high-LET) charged particles and for improved techniques of patient selection, treatment planning, and fractionation scheduling. This search will move to the new medical accelerator at the

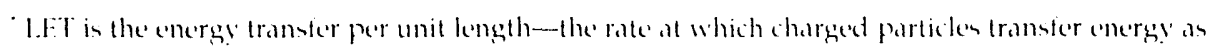
they interact with electrone when troveling through a medium. Difterent ioms have different i.f.Ts. 


\author{
Radiation Biology and \\ Biophysics Research
}

National Institute of Radiological Science in Chiba, Japan. The possibilities for additionally conducting this type of work at GSI in Darmstadt, Germany, with the assistance of Heidelberg University, are also being explored.

Improved techniques for treatment planning were pursued steadily at LBL and will be continued under $\mathrm{NCI}$ support. We have developed an excellent treatment planning system, which will soon incorporate full 3D planning as well as multiple scattering corrections. Members of LBL's radiation oncology group have also been heavily involved in the planning of a proton-therapy facility at the University of California-Davis Medical Center, as discussed in an earlier section of this chapter.

As part of a more-fundamental program of investigation, we have measured the dose-survival responses in i itro for $150-k V p x$-rays and for $425-$ $\mathrm{MeV} \mathrm{Ne}$ ions in each of three recurrent human tumor cell lines (with normal luman skin fibroblasts as a control) that had failed megavoltage x-ray therapy. We have also begun measuring micronuclei yields, since induction of micronuclei provides an early indication of genetic damage by radiation; prelliminary data in itro have correlated well with radiosensitivity. Potential uses of these assays include screening of tumor biopsies to identify candidates for charged-particle radiotherapy.

Radiation biology and biophysics research activities at the Bevalac address a broad range of scientific questions. This work is relevant to the missions of several national agencies as well as to the private sector. These scientific questions have been addressed by experiments employing a wide variety of beams, ranging from light ions such as helium to heavy ions such as lanthanum. These are a few highlights of their diverse and collaborative research programs.

NASA-funded biophysical research at the Bevalac has focused mainly on three space-related problems. First, an investigation is underway to assess the effects of heavy-particle fragmentation on cell killing and transformation (sidelar). In particular, the use of polyethylene or water-equivalent shielding material in space is under consideration, so quantitative information is necessary to determine what this material would do to the high-energy, heavy charged particles found in cosmic radiation, such as iron at $600 \mathrm{MeV} / \mathrm{n}$.

A varicty of more-fundamental work is conducted. One program examines and interprets the mutational spectra-the numbers and types of changes in DNA-in mamallian cells exposed to various charged particles, including $\mathrm{Fe}$, at high and low dose rates.

DOE and NASA have supported studies directed at understanding the RBE of high-LET radiations for inducing cancer. Using the Harderian gland of the mouse as the test system, tumor prevalence data are obtained for heavy ions ranging up to $953 \mathrm{keV} / \mathrm{mm}$ in LET. The subjects are irradiated and the prevalence of Harderian-gland tumors is examined 16 months later. A primary objective is the estimation of the initial slope of the dose-response curve for tumor prevalence, along with the LET dependence of the initiial slope of the curve. The ratio of this initial slope to that obtained with ${ }^{(1)} \mathrm{Co}$ gamma rays gives an estimate of the maximum RBE for radiogenic neoplasia. The tumor-prevalence data are also analyzed in terms of radiation fluence and the probability that any given cell will be traversed by an ion. Contrary to data on normal tissue responses and from cell transformation studies, no convincing evidence has been found that the RBE drops at very high LET values. These data have important implications for estimating the cancer risk from heavily ionizing radiation. 


\section{BEVALAC OPERATIONS}

In addition, dose-fractionation studies, which enable us to assess whether protracting a given particle dose reduces the hazard of tumor induction, have been completed. Early results indicate that fractionation does not spare the radiogenic cancer response--in other words, that small doses of heavy ions spread out over time can be as risky as a single dose of the same size. Methods are also being developed to determine cells at risk and the transformation rate for in virio irradiations.

In 1992, LBL was chosen as a site of NSCORT (NASA Specialized Center for Research and Training) in the field of Radiation Health. The major objective is basic and applied radiobiological research directly applicable to assessment of the particulate radiation risk associated with extended manned space missions. A second important objective is education to enhance the nation's pool of trained scientists. Colorado State University's Department of Radiation Health Sciences is our partner in this endeavor. The first class of five NSCORT students has embarked upon a training program at Colorado State, with research at LBL planned for summer 1993.

Significant progress was made by NSCORT in 1992 in several fields, including the study of enzymatic DNA repair processes and the examination of comparative mutagenesis in two cell systems, one human and one rodent. Transformation of mouse mammary epithelial cells has been quantified as part of an ongoing study of neoplastic progression. Applied NSCORT research is directed toward assessing the risk of radiation carcinogenesis through retroactive analysis of patients treated therapeutically at LBL with helium ions; these data will be compared with the extensive experimental data base on animal carcinogenesis. Extrapolation procedures for human risk analysis are being developed; this will make it easier to interpret results from one species in the context of another and to extrapolate from highto low-fluence data. Theoretical studies address particle-track structure and quantitative estimation of initial damage.

\section{Surviving Three Years in Space}

According to the calculations for one scenario involving three years outside the magnetosphere, any given cell within an astronaut will probably be hit many times by protons and helium ions. Heavier ions (with atomic number greater than 10), though less common, would still hit one in three cell nuclei, and would have a disproportionate effect. (The calculations refer to the omnidirectional background of galactic cosmic rays. They assume that the astronauts would have a place to take shelter from the intense but short-lived and directional radiation of solar flares.)

The total dose may be as much as one sievert, or 100 rem-much more than a U.S. radiation worker would be allowed to receive in the same period. Deducing the biological effects is a complex matter. Heavy ions can damage a cell either indirectly or directly. They can produce free radicals in water and thus cause a cascade of chemical reactions. The ions can also interact directly with the DNA in the cell nucleus. A cell can be killed outright or damaged in various ways, including DNA alteration.

Radiation-induced cell transformation, one of the more worrisome biological results, begins with molecular events in the DNA, such as deletions of genes, translocations, and other genetic rearrangements. In addition to being massive and thus carrying great energy, heavy ions deposit most of that energy at the "Bragg peak," a single point at the end of their path. There, extensive cellular damage occurs, including greater potential for breaking both strands in the double helix of DNA. This compromises the cell's primary method of repairing damaged DNA, which uses an intact strand as a template.

Additional knowledge on both the physical and the biological effects of radiation will be needed for spacecraft design as well. The simplest approach would be to include massive shielding, but this could backfire, at least for the amount of overall shielding that a mechanically and financially practical spacecraft could accommodate. Naively designed shielding might make matters worse by turning the incoming particle into a shower of fragments. It would be virtually impossible to build a shielding wall that stops all these secondary particles; thus the shielding must be designed to admit the least-harmful spectrum of radiation. Clearly, a mission to Mars will require new knowledge in many fields, including the effects of heavy-ion irradiation. Venues for this research in the wake of the Bevalac's closure have yet to be identified.

Based upon. an LBL Research Review article by leff Kalm of the Public Information Department. 


\section{Equipment}

Levelopment and Related Biomedical Research

Figure 6-8. One of the 1992 highlights in Bevalac biomedical technology development was an R\&D 100 award for the Raster Scanner Beam Delivery System. The Raster Scanner, commissioned for clinical use in 1991. It scans the rigid heavy-ion beam back and forth much like the electron beam in a television's picture tube. Biological measurements in the proximal peak of the field, using neon ions, have shown results as good as those of the "wobbler" beamdelivery system, itself a previous R\&D 100 winner. Innovations such as the Raster Scanner, especially in beam delivery systems, will offer excellent technology-transfer potential as medical accelerators are built and improved worldwide. We are seeking ways to keep the critical $R \& D$ teams together in the wake of the 1993 shutdown so that medical-acrelerator programs such as the UC-Davis proton therapy initiative can derive the greatest possible benefit from the expertise developed at the Bevalac.
The most important attribute of heavy charged-particle beams for radiotherapy is their dose-localizing property. We continue to develop the hard ware necessary to achieve the optimum dose delivery - that is, delivering a uniform dose within the entire tumor volume with minimum damage to adjacent healthy tissues. The most significant advance of recent years was the 1991 commissioning of the Raster Scanner for clinical use. The achievement was recognized in 1992 with an R\&D 100 award.

The Raster Scanner (Figure 6-8) represents an advance over systems currently in use, including the "wobbler" we developed in the mid-1980s. It scans the beam across the treatment area, much as the electron beam in a

\section{RASTER SCANNING SYSTEM}

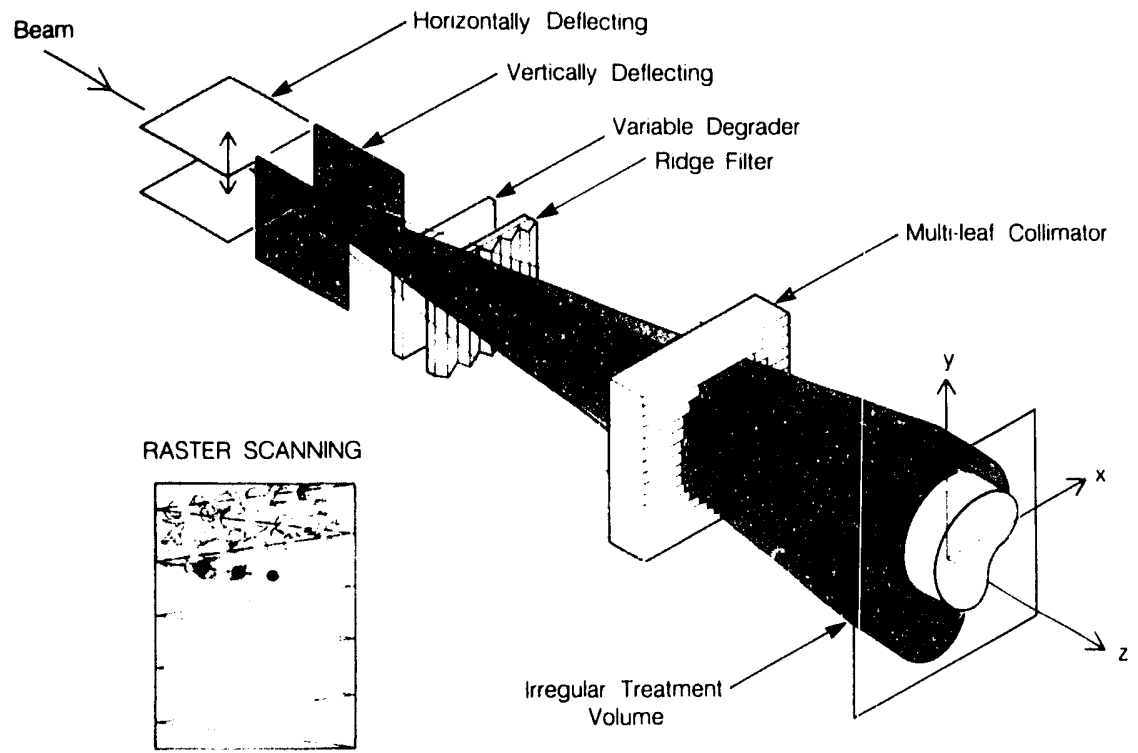

CBB 894-3647

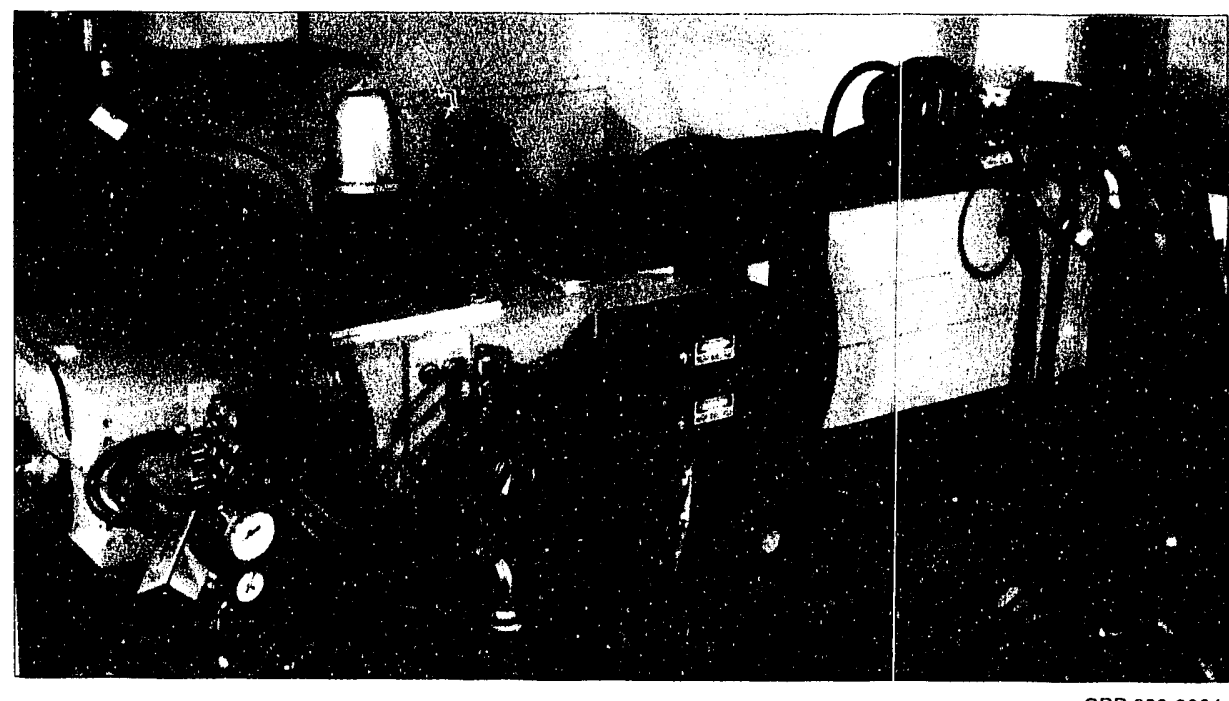

CBB $883-2684$ 
television's picture tube is scanned across the screen. Because the ion beams used at the Bevalac are so much more "rigid" than the low-energy electron beam in a picture tube, it was difficult to implement. Considerable effort has gone into its power supply and control system so that sharp-edged fields could be produced and the scan speed could be modulated to control the dose. Biological measurements in the proximal peak of the field, using neon ions, gave results as good as those of the wobbler.

Development of dynamic conformal treatments has continued with the construction of a multileaf collimator. We tested it with helium and neon ions for transmission through the gap between the fingers and found that leakage of the particles was suppressed sufficiently for use with patients. These results are a promising indication that the ultimate goal of implementing dynamic conformal particle therapy will be achieved.

\section{By and in Collaboration with Bevalac Staff}

A. Albergo, S. Costa, M. Maryeo, D). Nicotra, Ci. Poli, R. I'otenza, C. Ture, H.J. Crawford, and I. Flores, "losition sensitive scintillator disces for intermediate and high energy neutron spectroscopy," Nucl. Instrum. Meth. A 311, 280 (14492).

I.R. Alonso, "Accelerators in proton therapy," invited talk, Spring Meeting of the American Physical Sociely (Washington, $D$, 1942), Bull. Am. l'hys. Sox. 37, 2, p. 459 (I)42).

J.R. Alonso, "()ptimizing proton therapy at the I.BL. Medical Accelerater," final report, National Camoer Institute Cirant ( A $53835 ;$ : I.BL-320)53.

J.R. Alonso, "Proton therapy construction projects in the Lnited States," invited talk, 13th National Particle Accolerator Conference (Dubna, Russia, I042); I.B1-3.3211 .

I.R. Alomse, "Statum of the LC" Davis proton therapy project," l'article Therapy (i) Operative Group meeting PTC() $;$ XVII (loma linda, (A, I (N)2); abstracts to be published in "Particles" newsiletter, J. Sisterson, editor.

3.R. Akonso, "Summary of the workshop on technical systems for a protom therapy lacility," larticle Therapy (in-( )peralise

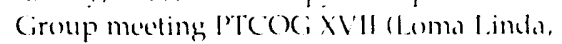
(A, (4)2); abstracts to be publisted in

"Particles" newsletter, I Sisterson, cditur.

J.R. Alonso, "The L.BL-UC Daris prolon therapy project," larticle Therapy ( (1)

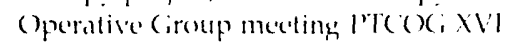

(Vancouver, Candada, 1942); abstracts published as supplement to "Particles" new'sletter, issue 10, J. Sisterson, edilor.

J.R. Alonse, "20 years of production and use of radionetive nuclear beams at the Bevalac," invited lalk, American Chemical Sociely mecting (San franciso, CA, 144)2).

I.R. Nlonse, W.T. Chu, B. Foinberg, B.IV. Ludewigt, T.R. Renner, and I.W. Staples, "Future biomedical research at the Bevialace," in Proctelimis of the NIRS Inlermational Workshop on l lealy Charged l'artick Therapy and Relaled Subjects, $A$. Itane and T. Komni, editors, 201-20)2 (Chiba, Japan,

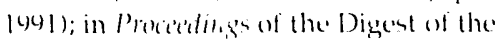
World Congrese on Vedical Physios and Biomedical Enginering (K! ioto, Japan, July 1941); Medical and Biological Engineering and Computing 29, Suppl. Part 1, 51 (|49)|).

I.R. Alonse, T.R. Remner, W.T. Chu, B. Ludewigt, P.A. Petti, R.P'. Singh, and M.A. Nymun, "l.ine scomming: an efficacious technique for three-dimensional treatment delisery of charged-particle beoms," in

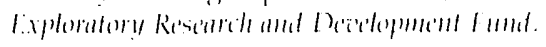

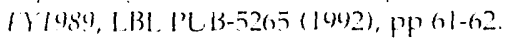

I.R. Cistro, l'I. Pelli, W.T. Chu, I.M. Collier, 1. Daftari, S. Pitluck, T. Renner, B. l.udewigh, and lE. Blakeley, "Potential of heary charged-particle radtiotherapy uning a new dynamic conformal techniçue," in Procerding of the Anntal Meeting of the Radiation Rescarch Society (144)2).

I.R. Costro, P'.1. l'etti, W. Chu, I.M. Collier, 1. Dallari, S. Pilluch, T. Renner, B. I udewigh, and I:. Blakely, "Polential of

\section{Publications and Presentations}


heary charged-particle radiotherapy using a new dynamic conformal technicue," in Procerdings of the ASTRO meeting (November 1991).

C.M. Colata, M.J. Bennett, B. Foinberg, T.K. McGathen, and M.A. Nyman, "Improvement of the Bevalac extracted beam time structure for slow (9.5 sec.) extraction," Bull. Am. Phys. Soc. 37, 888 (1942).

S. Chattopadhyay, K.-1. Kim, R. Byrns, R. Donahue, I. Edighoffer, R. Gough, E. Hover, W. Leemans, J. Staples B. Taylor, and $M$. Xie, "Design of a superconducting linear accelerator for an infrared free electron laser of the proposed chemical denamies research laboratory at L.BL.," in Priverettinges of the 1942 Linear Accelerator Conference, 23-28 (Othawa, Camada, l(s)2).

W.T. Chu, B.A. Ludewigh, and T. Renner, "Specifications for the heavy chargedparticles radiotherapy facility," Bull. Am. Phys. Soc. 37, 984t (1942).

W.T. Chu, B.A. Ludewigh, K.M. Marks, M.A. Nyman, T.R. Renner, R.P. Singh, and R. Stradtner, "Three-dimensional conformal therapy using light-ion beams," in Procededinse of the Digest of the World Congress on Medical Physics and Biomedical lengineering (Kvoto), Japan, July |col); Medical and Biological Engineering and Compuling 29. Suppl. Part 1,770 (1991).

(i. Coutrakon, M. Bauman, D. Lessina, D). Miller, J. Nusbaum, ]. Slater, J. Johamning, 1. Miranda, I'. Doldaca, J. Siebers, and B. l.udewigt. "A prototype berm delivery system for proton medical accelerator at Loma Linda," Med. Phy's. 18, $1(14) 3(104) 1)$.

H.). Crawtord, V.F. Dudkin, F.F. Kovalew, N.A. Nefedor, Y.A. Antonchik, S.D. Bogdanor, Y'I. Ostroumos, and E.L. Benton, "Target frogments in collisions of 1.8 Civ/N Fe nuclei with photeremulsion nucled and ciscade exaporation model," Nucl. Phys. A 530, 750 (1940).

T. Doke, H.l. Crawford, I.N. Engeldge, 1. Flores, L. Greiner, T. Kashiwagi, I. Kikuchi, K. Masuda, K. Nishijima, E. Shibamura, and T.J.M. Symons, "Sampling calorimeter for high energy heavy particles filled with allene-doped liquid argen," Nucl. Instrum. Meth. A 302, 24(1)(140)1).

B. Foinberg, H. Ciculd, W.E. Meverhot, A. Belkacem, H.-l'. Muilskitter, I.R. Alonso, 1..
Blumenfeld, l:. Dillard, N. Guardala, G.F. Krobs, M.A. McMahan, M.J. RhordesBrown. B.S. Rude, I. Schweppe, D.W. Spooner, K. Street, P. Thieberger, and $H$. Wegner, "Relativistic electron and proten impact ionization of highly stripped heavy ions determined from projectile electron loss in 112 and He," Bull. Am. Phys. Soc. 37. $1(184(154) 2)$.

1. Flores, l'. Blando, H.J. Crawtord, S. Ingelage, L. Creiner, S. Ko, C.F. Krebs, and G. Verser, "A four channol $250 \mathrm{MH} 1 \%$ visual counter," I.BB. PUB-3(1)97 (1942).

I.E. Ciondinght and J.R. Alonso, "Proton therapy research and treatment center," L.BL. I'U B-5.348 (194)2).

N. Hasehe, T. Doke, T. Kashiwagi, S Kalaoka, J. Kikuchi, H. Moriya, K. Nishijima, and H.J. Crawford, "Iosition linearity and resolution of large positionsensitive silicon detecter with a highly uniform thickne'ss," Ipn. 1. Appl. Phys 31, ||$\varphi \mid(\mid 092)$.

I.C. Kalnins, Ci.t. Krebs, M. Tekawia, D. Cowles, and T. Byrne, "Bevalac extration," LBI. -31967 (1962).

W'.l. Kehoc, A.C. Mignerey, A. Moroni, I. Iori, C.F. I'enslee, N. Colomna, K. Hanold, D.R. Bow'man, L.C. Moretto, M.A.

MoWahan, I.T. Walton, and G.J. Worniak, "A medular array to detect complex iragments produced in intermediate-energy reverse-kinematic reactions," Nucl. Instrum. Meth. A 311, 258 (1492); L.BL-310(15 (1991).

K.-J. Kim, R. Byrns, S. Chattopadhyay, R. Donahue, I. Edighoffer, R. Gough, E. Hoyer, W. Leemans, I. Staples, B. Taylor, and $M . X i c$, "An infrared free electron laser sistem for the proposed chemical dynamics research laboratory at L.BL based on a 500$)$ MHz superonducting LIINAC," in Prencetimes of the 13 th lnternational FFE. Conference, 23-2s (Kube, Japan, 14)2); L.B1.$322 \times 7$.

R. Mader, M. Eladsar, W.M. Zhang, I. Schumbach, D. Keome, B.D. Anderson, A.R. Baldwin, J.W. Watson, C.F. Krebs, H. Wieman, G.1). Westlall, C. Gale, and $k$. Haglin, "l'robing the nuclear matter equation of state with neutrons from nucleus-nucleus collisions," Nuctear Physics A, in Procedimes: of the Intermational Nuclear Physics conference, adited by R. Bock (Wiesbaden, (iermans, luly /(4)2). 


\section{BEVALAC OPERATIONS}

K. Matsuta A Crawa, Y. Neri, T. Minumiromo, M. Fukuda, A. Kitagawa, T. Ohtsutx, S. Momota, S. Fukusta, Y. Matsuo, H. Takerhi. 1. Minami, K. Sugimoto, I. Tanihata, K. Omata, S. Shimoura, J.R. Alonso, G.F. Krobs, and T.I.M. Symons, "A tragment sefarater at 1.BI for the ineta-NMR eperiment," Nucl. Instrum. Weth. B 70 . 344-308 (1402); in Proceitms of the and International Conference on Radionctive Vuclear Beams (Lousain-la-Neuve, Belgium, 1491), IOP Publishing LId. (1992); 1.BL-32077 (1992).

K. Matsuta, A. Ozawa, I. 'ajiri, T. Minamisono, M. Fukuda, A. Kitagawa, $\mathbf{5}$. Momota, T. Ohtsubo, Y. Matsuu, $H$. Takechi, S. Fukuda, I. Minami, K. Sugimoto. 1. Tanihata, K Omata, I.R. Alonso, G.F. Krebs, and T.I.M. Simons. "Obsersation of spin polarization of projectile fragments from Iin Mel t"Ca - Au vollisions," Phis. Lett. B 281. $21+11442) ; 1 . B 1.3211 ; 9(1442)$

K. Statulta, A. Orawa, Y. Nori, T Minamiuno, if. Fuhuda, A Kitagawa, s. Momota, T. Ohtsubx, Y. Matsuo, H. Takeechi, 5 Fukuda, I. Minami, K. Sugimoto, I. Tanihata. K. Omata, J.K. Alonso, Ci.f. Krebs, and T.J.M Simous. "Polarization phenomena in provectile tragmentition proxems,"

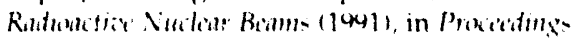
of the Ind International Conference on Radicacitve Nuclear Beams, Tl. Delbar. editor itonusain-la-Neure, Belgium, (491), Pp. 361-34x; 1.B1-32017\% 11442)

K. Matsuta, A (Yawa, ) Vopro, T

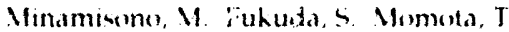
Ohtsutres Futuda, K. Sugimote. 1 Tanihata. $K$. Yonhida. K. Omata. I. $R$ Alones, Gif. Krebs and T.l.M simons, "Fragment polariation of "ist in the " ${ }^{\text {t" }} \mathrm{T} i+\mathrm{C}$ coilision." In Procctedmen of the 4th International Conterence on Hepertine Interactions (C)aha, lapan, 1442)

K. Matsuta, A. O/awa, Y Noniri, T

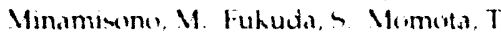

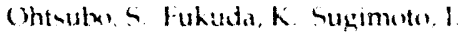

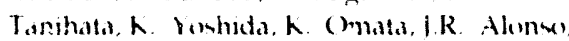

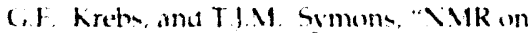

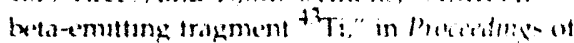
the nth Internatienal Conference on >ucles Far trom stabilite and the oth Internaturnat conterence on Atoms Mame and Fundamental (andant- (ikernhanted)-Kuen.

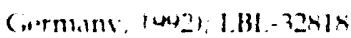

1.D. Molituris, W.E. Meverhor, Ch. Stoller, R. Anholt, D.W. Spoomer, L.C. Moretto, L.Ci, Sxolvotka, R.l. MCDonald, Ci.l. Wozniak, M.A. MoMahan, L. Blumenfeld, $N$. Colonna, M. Nessi, and E. Morenzoni, "Molecular-orbital study of late-fission times in deep inelastic ${ }^{238} \mathrm{U}+{ }^{23 k} \mathrm{U}$ collisions," submitted to Phys. Rev. Lett. (1992).

D.I. Olson M. Baumgartner, D.E. Greiner, P.J. Lindstrom, T.J.M. Symons, R. Wada, M.L. Webb, B.L. Berman, H.J. Crawford, and J. Engelage, "Direct observation of the giant dipole resonance of ${ }^{16} \mathrm{O}$ via electromagnetic dissociation," Nucl. Phys. B 24 (suppl.), 157 (1991).

L. Phair, W. Bauer, D.R. Bowman, $N$. Carin, R.T. de Souza, C.K. Gelbke, W.G. Gong, Y.D. Kim. M.A. Lisa, W.G. Lynch, G.F. Peaslee, M.B. Tsang, C. Williams, F. Zhu, $X$. Colonna, K. Hanold, M.A. MCMahan, G.J. Wozniak, and L.G. Moretto, "Multifragment emission in "3h Ar +197Au and ${ }^{124} \mathrm{Xe}+{ }^{4}: \mathrm{Au}$ collisions. Percolation Medel," Phys. Lett. B 285, $10(1992)$.

B.M. Rabin, J.A. Joseph, WV.A. Hunt, S.B. Kandasamy, and B. Ludewigt, "Behavioral endpoints for radiation injury, in Prociedings: of the World space Congress (COSPAR) (Washingtom, DC, 1992).

T.R. Renner, B.A. Ludewigt, and W.T. Chu, "Saftety issuen involving the accelerated heas." Garged particles for radiotherapy:" Bull. Am. Phys Sxic. 37, 984 (1992)

P. Roussel-Chomaz, N. Colonna, Y. Blumenteld, B. Libbr, G.F. Peaslee, D.X. Delis, K. Hanold, M.A. Mo Mahan, I.C. Neng, Q.C. Sui, G.). Worniak, and L.G. Moretto. "Complex tragment production and multitragmentation in ${ }^{84} 1 . a-i n d u c e d$ reaction at $35,41,45$, and $55 \mathrm{Mel}$, s." submitted to vicl Phis A (1992); LBL-32433 (1992).

1. Staples, I. Fdighuttor, and K-I. Kim, "Flectron beam transport for the I.BI. IRFi.," in Prectednis of the 1442 linear Accelerate r Conferemo, $23-28$ iottawa. Canada, 1442).

R.K. Sun, C.F. Kreter, A.R Smith, and H.H. Hou, A neutron done detecter with REM response wo I Ceel', 1. B1-3234? (1442) 

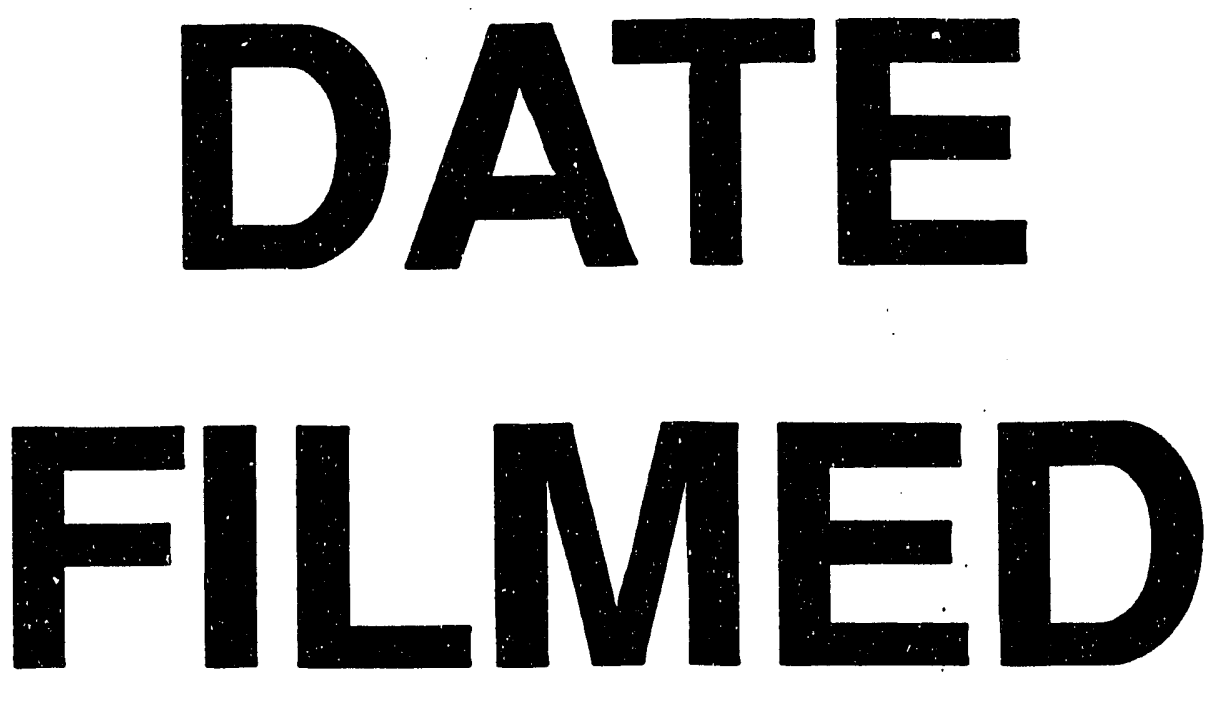

$9 / 22 / 93$
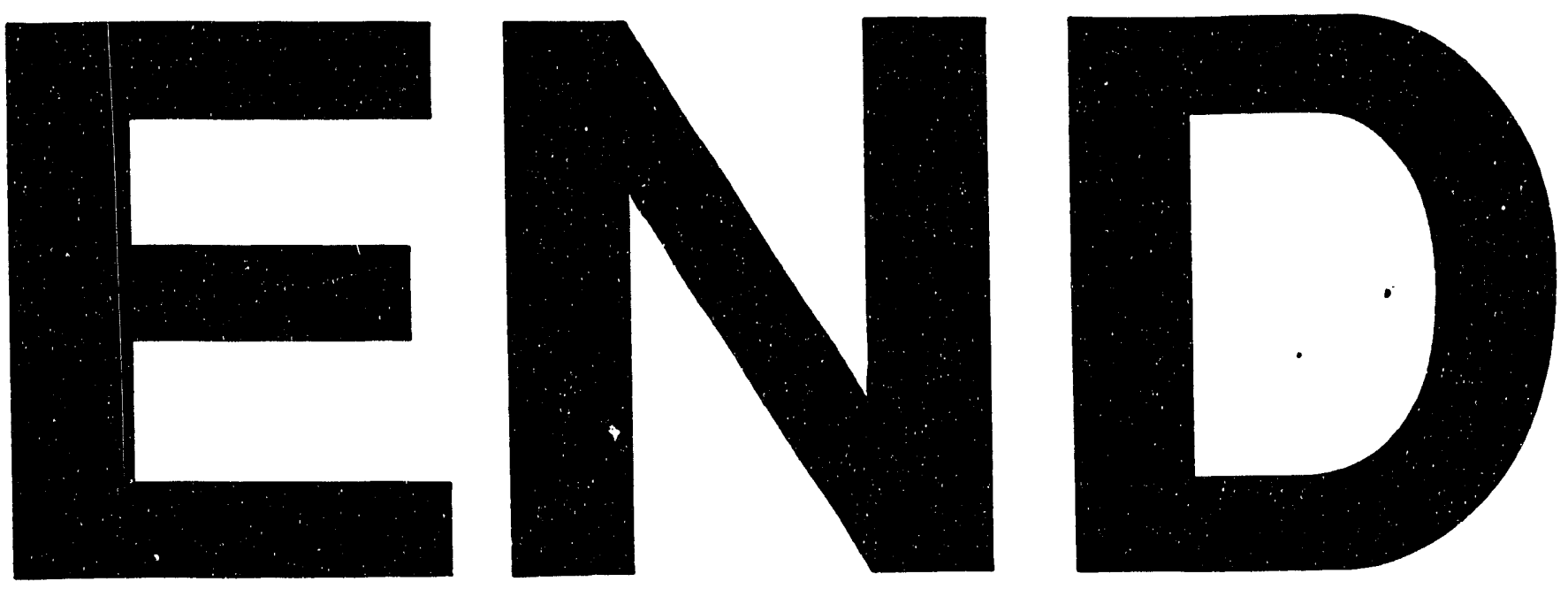
